\title{
On the formation of trapped surfaces
}

by

\author{
Sergiu Klainerman \\ Princeton University \\ Princeton, NJ, U.S.A.
}

\author{
IGOR RODNIANSKI \\ Princeton University \\ Princeton, NJ, U.S.A.
}

\section{Introduction}

\subsection{Main goals}

In a recent important breakthrough D. Christodoulou [C] has solved a long standing problem of general relativity of evolutionary formation of trapped surfaces in the Einsteinvacuum space-times. He has identified an open set of regular initial conditions on a finite outgoing null hypersurface leading to a formation of a trapped surface in the corresponding vacuum space-time to the future of the initial outgoing hypersurface and another incoming null hypersurface with prescribed Minkowskian data. He also gave a version of the same result for data given on part of past null infinity. His proof, which we outline below, is based on an inspired choice of the initial condition, an ansatz which he calls short pulse, and a complex argument of propagation of estimates, consistent with the ansatz, based, largely, on the methods used in the global stability of the Minkowski space [CK]. Once such estimates are established in a sufficiently large region of the space-time, the actual proof of the formation of a trapped surface is quite straightforward.

The goal of the present paper is to give a simpler proof by enlarging the admissible set of initial conditions and, consistent with this, relaxing the corresponding propagation estimates just enough that a trapped surface still forms. We also reduce the number of derivatives needed in the argument from two derivatives of the curvature to just one. More importantly, the proof, which can be easily localized with respect to angular sectors, has the potential for further developments. We prove in fact another result, concerning the formation of pre-scarred surfaces, i.e. surfaces whose outgoing expansion is negative in an open angular sector. We only concentrate here on the finite problem, the problem from past null infinity can be treated in the same fashion as in $[\mathrm{C}]$ once the finite problem

S. K. was supported in part by the NSF grant DMS-0901250. I. R. was supported in part by the NSF grant DMS-0702270. 
is well understood. The problem from past null infinity has been subsequently considered in a recent preprint by Reiterer and Trubowitz, $[\mathrm{RT}]$.

We start by providing the framework of double null foliations in which the result of Christodoulou is formulated. We then present, in $\S 1.3$, the heuristic argument for the formation of a trapped surface. In $\S 1.4$ we then introduce Christodolou's short-pulse ansatz and discuss the propagation estimates which it entails.

\subsection{Double null foliations}

We consider a region $\mathcal{D}=\mathcal{D}\left(u_{*}, \underline{u}_{*}\right)$ of a vacuum space-time $(M, g)$ spanned by a double null foliation generated by the optical functions $(u, \underline{u})$ increasing towards the future, $0 \leqslant u \leqslant u_{*}$ and $0 \leqslant \underline{u} \leqslant \underline{u}_{*}$. We denote by $H_{u}$ the outgoing null hypersurfaces generated by the level surfaces of $u$ and by $\underline{H}_{\underline{u}}$ the incoming null hypersurfaces generated by the level hypersurfaces of $\underline{u}$. We write $S_{u, \underline{u}}=H_{u} \cap \underline{H}_{\underline{u}}$ and denote by $H_{u}^{\left(\underline{u}_{1}, \underline{u}_{2}\right)}$ and $\underline{H}_{\underline{u}}^{\left(u_{1}, u_{2}\right)}$ the regions of these null hypersurfaces defined by $\underline{u}_{1} \leqslant \underline{u} \leqslant \underline{u}_{2}$ and $u_{1} \leqslant u \leqslant u_{2}$, respectively. Let $L$ and $\underline{L}$ be the geodesic vector fields associated with the two foliations and define

$$
\frac{1}{2} \Omega^{2}=-g(L, \underline{L})^{-1}
$$

Observe that the flat value $\left({ }^{1}\right)$ of $\Omega$ is 1 . As is well known, our space-time slab $\mathcal{D}\left(u_{*}, \underline{u}_{*}\right)$ is completely determined (for small values of $u_{*}$ and $\underline{u}_{*}$ ) by data along the null, characteristic, hypersurfaces $H_{0}$ and $\underline{H}_{0}$ corresponding to $\underline{u}=0$ and $u=0$, respectively. Following [C] we assume that our data is trivial along $\underline{H}_{0}$, i.e. assume that $H_{0}$ extends for $\underline{u}<0$ and that the space-time $(M, g)$ is Minkowskian for $\underline{u}<0$ and all values of $u \geqslant 0$. Moreover we can construct our double null foliation such that $\Omega=1$ along $H_{0}$, i.e.

$$
\Omega(0, \underline{u})=1, \quad 0 \leqslant \underline{u}^{*} \underline{u}_{*} .
$$

Throughout this paper we work with the normalized null pair $\left(e_{3}, e_{4}\right)$, with

$$
e_{3}=\Omega \underline{L}, \quad e_{4}=\Omega L \quad \text { and } \quad g\left(e_{3}, e_{4}\right)=-2
$$

Given a 2 -surface $S(u, \underline{u})$ and an arbitrary frame $\left(e_{a}\right)_{a=1,2}$ tangent to it, we define the Ricci coefficients

$$
\Gamma_{(\lambda)(\mu)(\nu)}=g\left(e_{(\lambda)}, D_{e_{(\nu)}} e_{(\mu)}\right), \quad \lambda, \mu, \nu=1,2,3,4
$$

$\left({ }^{1}\right)$ Note that our normalization for $\Omega$ differs from that of $[\mathrm{KN}]$. 
These coefficients are completely determined by the following components:

$$
\begin{array}{rlrl}
\chi_{a b} & =g\left(D_{a} e_{4}, e_{b}\right), \quad \underline{\chi}_{a b}=g\left(D_{a} e_{3}, e_{b}\right), \\
\eta_{a} & =-\frac{1}{2} g\left(D_{3} e_{a}, e_{4}\right), \quad \underline{\eta_{a}}=-\frac{1}{2} g\left(D_{4} e_{a}, e_{3}\right), \\
\omega & =-\frac{1}{4} g\left(D_{4} e_{3}, e_{4}\right), \quad \underline{\omega}=-\frac{1}{4} g\left(D_{3} e_{4}, e_{3}\right), \\
\zeta_{a} & =\frac{1}{2} g\left(D_{a} e_{4}, e_{3}\right), & &
\end{array}
$$

where $D_{a}=D_{e_{(a)}}$. We also introduce the null curvature components

$$
\begin{aligned}
\alpha_{a b} & =R\left(e_{a}, e_{4}, e_{b}, e_{4}\right), & \underline{\alpha}_{a b} & =R\left(e_{a}, e_{3}, e_{b}, e_{3}\right), \\
\beta_{a} & =\frac{1}{2} R\left(e_{a}, e_{4}, e_{3}, e_{4}\right), & \underline{\beta}_{a} & =\frac{1}{2} R\left(e_{a}, e_{3}, e_{3}, e_{4}\right), \\
\varrho & =\frac{1}{4} R\left(L e_{4}, e_{3}, e_{4}, e_{3}\right), & \sigma & =\frac{1}{4}{ }^{*} R\left(e_{4}, e_{3}, e_{4}, e_{3}\right) .
\end{aligned}
$$

Here ${ }^{*} R$ denotes the Hodge dual of $R$. We denote by $\nabla$ the induced covariant derivative operator on $S(u, \underline{u})$, and by $\nabla_{3}$ and $\nabla_{4}$ the projections to $S(u, \underline{u})$ of the covariant derivatives $D_{3}$ and $D_{4}$, respectively, see precise definitions in [KN]. Observe that

$$
\begin{aligned}
\omega & =-\frac{1}{2} \nabla_{4} \log \Omega, \quad \underline{\omega}=-\frac{1}{2} \nabla_{3} \log \Omega, \\
\eta_{a}=\zeta_{a}+\nabla_{a} \log \Omega, & \underline{\eta}_{a}=-\zeta_{a}+\nabla_{a} \log \Omega .
\end{aligned}
$$

The connection coefficients $\Gamma$ satisfy equations which have, very roughly, the form

$$
\nabla_{4} \Gamma=R+\nabla \Gamma+\Gamma \Gamma \quad \text { and } \quad \nabla_{3} \Gamma=R+\nabla \Gamma+\Gamma \Gamma
$$

Similarly, the Bianchi identities for the null curvature components satisfy, also very roughly,

$$
\nabla_{4} R=\nabla R+\Gamma R \quad \text { and } \quad \nabla_{3} R=\nabla R+\Gamma R .
$$

The precise form of these equations is given in $\S 3$, see (3.1)-(3.4). Among these equations we note the following two, which play an essential role in Christodoulou's argument for the formation of trapped surfaces:

$$
\begin{aligned}
\nabla_{4} \operatorname{tr} \chi+\frac{1}{2}(\operatorname{tr} \chi)^{2} & =-|\widehat{\chi}|^{2}-2 \omega \operatorname{tr} \chi \\
\nabla_{3} \widehat{\chi}+\frac{1}{2}(\operatorname{tr} \underline{\chi}) \hat{\chi} & =\nabla \widehat{\otimes} \eta+2 \underline{\omega} \hat{\chi}-\frac{1}{2}(\operatorname{tr} \chi) \underline{\hat{\chi}}+\eta \widehat{\otimes} \eta
\end{aligned}
$$



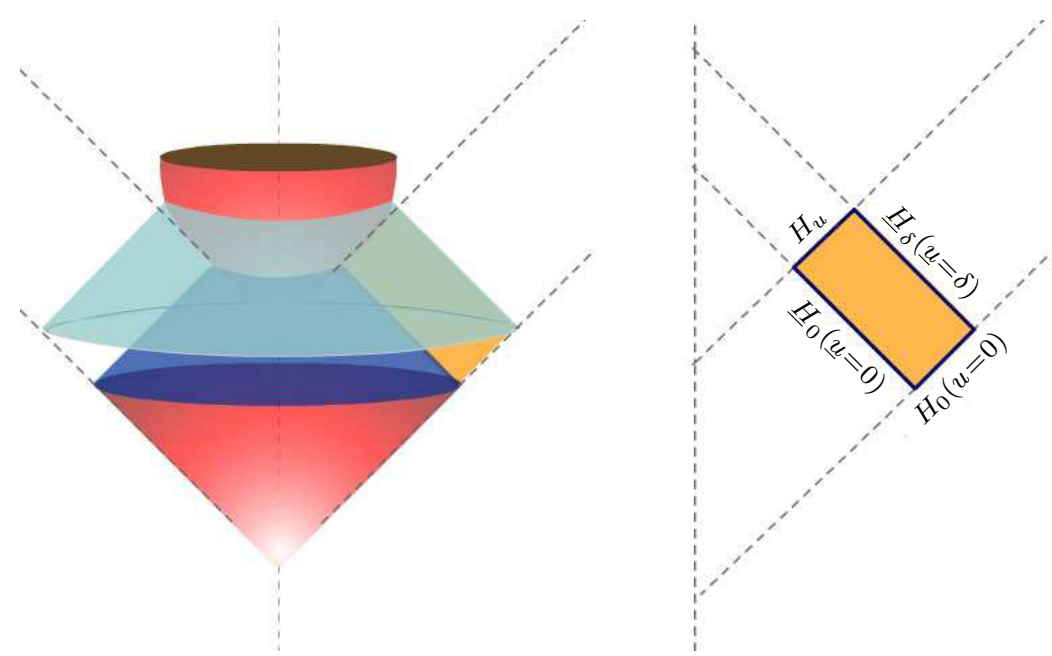

Figure 1. The highlighted region on the right represents the domain $\mathcal{D}(u, \underline{u}), 0 \leqslant \underline{u} \leqslant \delta$. The same picture is represented, more realistically on the left. The lower region on the left is the flat portion of $H_{0}, u=0$, while the upper region, corresponding to large values of $u$, is trapped starting with $\underline{u}=\delta$.

\subsection{Heuristic argument}

We start by making some important simplifying assumptions. As mentioned above we assume that our data is trivial along $\underline{H}_{0}$, i.e. assume that $H_{0}$ extends for $\underline{u}<0$ and that the space-time $(M, g)$ is Minkowskian for $\underline{u}<0$ and all values of $u \geqslant 0$. We introduce a small parameter $\delta>0$ and restrict the values of $\underline{u}$ to $0 \leqslant \underline{u} \leqslant \delta$, i.e. $\underline{u}_{*}=\delta$.

We also assume that the following conditions hold in the entire slab $\mathcal{D}(u, \delta)$. We denote by $r=r(u, \underline{u})$ the radius of the 2 -surface $S=S(u, \underline{u})$, i.e. $|S(u, \underline{u})|=4 \pi r^{2}$. We denote by $r_{0}$ the value of $r$ for $S(0,0)$, i.e. $r_{0}=r(0,0)$.

- For small $\delta, u$ and $\underline{u}$ are comparable with their standard values in flat space, i.e. $u \approx \frac{1}{2}\left(t-r+r_{0}\right)$ and $\underline{u} \approx \frac{1}{2}\left(t+r-r_{0}\right)$. We also assume that $\Omega \approx 1$ and $d r / d u \approx-1$.

- We assume that $\operatorname{tr} \chi$ is close to its value in flat space, i.e. $\operatorname{tr} \chi \approx-2 / r$.

- We assume that the term $E=\nabla \widehat{\otimes} \eta+2 \underline{\omega} \hat{\chi}-\frac{1}{2}(\operatorname{tr} \chi) \underline{\hat{\chi}}+\eta \widehat{\otimes} \eta$ on the right-hand side of equation (1.9) is sufficiently small and can be neglected in a first approximation. We assume also that we can neglect the term $(\operatorname{tr} \chi) \omega$ on the right-hand side of (1.8).

Given these assumptions we can rewrite (1.8) as

$$
\frac{d}{d \underline{u}} \operatorname{tr} \chi \lesssim-|\widehat{\chi}|^{2}
$$

or, integrating, as

$$
\operatorname{tr} \chi(u, \underline{u}) \lesssim \operatorname{tr} \chi(u, 0)-\int_{0}^{\underline{u}}|\widehat{\chi}|^{2}\left(u, \underline{u}^{\prime}\right) d \underline{u}^{\prime}=\frac{2}{r(u, 0)}-\int_{0}^{\underline{u}}|\widehat{\chi}|^{2}\left(u, \underline{u}^{\prime}\right) d \underline{u}^{\prime} .
$$


Multiplying (1.9) by $\widehat{\chi}$, we deduce that

$$
\frac{d}{d u}|\widehat{\chi}|^{2}+(\operatorname{tr} \underline{\chi})|\widehat{\chi}|^{2}=\widehat{\chi} E
$$

or, in view of our assumptions for $\operatorname{tr} \underline{\chi}$ and $d r / d u$,

$$
\begin{aligned}
\frac{d}{d u}\left(r^{2}|\widehat{\chi}|^{2}\right) & =r^{2} \frac{d}{d u}|\widehat{\chi}|^{2}+2 r \frac{d r}{d u}|\widehat{\chi}|^{2}=r^{2}|\widehat{\chi}|^{2}\left(-\operatorname{tr} \underline{\chi}+\frac{2}{r} \frac{d r}{d u}\right)+r^{2} \widehat{\chi} E \\
& =r^{2}|\widehat{\chi}|^{2}\left(-\left(\operatorname{tr} \underline{\chi}+\frac{2}{r}\right)+\frac{2}{r}\left(1+\frac{d r}{d u}\right)\right)+r^{2} \widehat{\chi} E=: F,
\end{aligned}
$$

that is

$$
r^{2}|\widehat{\chi}|^{2}(u, \underline{u})=r^{2}(0, \underline{u})|\widehat{\chi}|^{2}(0, \underline{u})+\int_{0}^{u} F\left(u^{\prime}, \underline{u}\right) d u^{\prime} .
$$

Therefore, as $\int_{0}^{u}|F| d u^{\prime}$ is negligible in $\mathcal{D}$, we deduce that

$$
r^{2}|\widehat{\chi}|^{2}(u, \underline{u}) \approx r^{2}(0, \underline{u})|\widehat{\chi}|^{2}(0, \underline{u}) .
$$

We now freely prescribe $\widehat{\chi}$ along the initial hypersurface $H_{0}^{(0, \delta)}$, i.e.

$$
\widehat{\chi}(0, \underline{u})=\widehat{\chi}_{0}(\underline{u})
$$

for some traceless 2-tensor $\widehat{\chi}_{0}$. We deduce that

$$
|\widehat{\chi}|^{2}(u, \underline{u}) \approx \frac{r^{2}(0, \underline{u})}{r^{2}(u, \underline{u})}\left|\widehat{\chi}_{0}\right|^{2}(\underline{u})
$$

or, since $|\underline{u}| \leqslant \delta$ and $r(u, \underline{u})=r_{0}+\underline{u}-u$,

$$
|\widehat{\chi}|^{2}(u, \underline{u}) \approx \frac{r_{0}^{2}}{\left(r_{0}-u\right)^{2}}\left|\widehat{\chi}_{0}\right|^{2}(\underline{u}) .
$$

Thus, returning to (1.10),

$$
\operatorname{tr} \chi(u, \underline{u}) \leqslant \frac{2}{r_{0}-u}-\frac{r_{0}^{2}}{\left(r_{0}-u\right)^{2}} \int_{0}^{\underline{u}}\left|\widehat{\chi}_{0}\right|^{2}\left(\underline{u}^{\prime}\right) d \underline{u}^{\prime}+\text { error. }
$$

Hence, for small $\delta$, the necessary condition to have $\operatorname{tr} \chi(u, \underline{u}) \leqslant 0$ is

$$
\frac{2\left(r_{0}-u\right)}{r_{0}^{2}}<\int_{0}^{\delta}\left|\widehat{\chi}_{0}\right|^{2} d u^{\prime} .
$$

Analyzing equation (1.8) along $H_{0}$, we easily deduce that the condition for the initial hypersurface $H_{0}$ not to contain trapped hypersurfaces is

i.e. we are led to prescribe $\widehat{\chi}_{0}$ such that

$$
\int_{0}^{\delta}\left|\widehat{\chi}_{0}\right|^{2} d u^{\prime}<\frac{2}{r_{0}}
$$

$$
\frac{2\left(r_{0}-u\right)}{r_{0}^{2}}<\int_{0}^{\delta}\left|\widehat{\chi}_{0}\right|^{2} d u^{\prime}<\frac{2}{r_{0}} .
$$

We thus expect, following Christodoulou, that trapped surfaces may form if (1.12) is satisfied. 


\subsection{Short-pulse data}

To prove such a result however we need to check that all the assumptions we made above can be satisfied. To start with, the assumption (1.12) requires, in particular, an $L^{\infty}$ upper bound of the form

$$
\left|\widehat{\chi}_{0}\right| \lesssim \delta^{-1 / 2}
$$

If we can show that such a bound persists in $\mathcal{D}$ then, in order to control the error terms $F$ we need, for some $c>0$,

$$
\begin{gathered}
\operatorname{tr} \underline{\chi}+\frac{2}{r}=O\left(\delta^{c}\right), \quad \frac{d r}{d u}+1=O\left(\delta^{c}\right), \quad \eta=O\left(\delta^{-1 / 2+c}\right), \\
\omega=O\left(\delta^{-1+c}\right) \quad \text { and } \quad \nabla \eta=O\left(\delta^{-1 / 2+c}\right) .
\end{gathered}
$$

Other bounds will however be needed, as we have to take into account all null structure equations. We face, in particular, the difficulty that most null structure equations have curvature components as sources. Thus we are obliged to derive bounds not just for all Ricci coefficients $\chi, \omega, \eta, \underline{\chi}, \underline{\omega}$ and $\underline{\eta}$ but also for all null curvature components $\alpha$, $\beta, \varrho, \sigma, \underline{\alpha}$ and $\underline{\beta}$. In his work [C] Christodoulou has been able to derive such estimates starting with an ansatz (which he calls short pulse) for the initial data $\widehat{\chi}_{0}$. More precisely he assumes, in addition to the triviality of the initial data along $\underline{H}_{0}$, that $\widehat{\chi}_{0}$ satisfies, relative to coordinates $\underline{u}$ and transported coordinates $\omega$ along $H_{0}$ (i.e. transported with respect to $d / d \underline{u})$,

$$
\widehat{\chi}_{0}(\underline{u}, \omega)=\delta^{-1 / 2} f_{0}\left(\delta^{-1} \underline{u}, \omega\right)
$$

where $f_{0}$ is a fixed traceless, symmetric $S$-tangent 2 -tensor along $H_{0}$. This ansatz is consistent with the following more general condition, for a sufficiently large number $N$ of derivatives and a sufficiently small $\delta>0$ :

$$
\delta^{1 / 2+k}\left\|\nabla_{4}^{k} \nabla^{m} \widehat{\chi}_{0}\right\|_{L^{2}(0, \underline{u})}<\infty, \quad 0 \leqslant k+m \leqslant N \text { and } 0 \leqslant \underline{u} \leqslant \delta .
$$

Notation. Here $\|\cdot\|_{L^{2}(u, \underline{u})}$ denotes the standard $L^{2}$ norm for tensor fields on $S(u, \underline{u})$. Whenever there is no possible confusion we will also denote these norms by $\|\cdot\|_{L^{2}(S)}$. We shall also denote by $\|\cdot\|_{L^{2}(H)}$ and $\|\cdot\|_{L^{2}(\underline{H})}$ the standard $L^{2}$ norms along the null hypersurfaces $H=H_{u}$ and $\underline{H}=\underline{H}_{\underline{u}}$, respectively.

Remark 1.1. In [C] Christodoulou also includes weights, depending on $|u|$, in his estimates. These allow him to derive not only a local result but also one with data at past-null infinity. In our work here we only concentrate on the local result, for $|u| \lesssim 1$, and thus drop the weights. 
Assumption (1.15), together with the null structure equations (1.6) and null Bianchi equations (1.7) leads to the following estimates for the null curvature components, along the initial null hypersurface $H_{0}$ :

$$
\delta\|\alpha\|_{L^{2}\left(H_{0}\right)}+\|\beta\|_{L^{2}\left(H_{0}\right)}+\delta^{-1 / 2}\|(\varrho, \sigma)\|_{L^{2}\left(H_{0}\right)}+\delta^{-3 / 2}\|\underline{\beta}\|_{L^{2}\left(H_{0}\right)}<\infty
$$

Consistent with (1.15), the angular derivatives of $\alpha, \beta, \varrho, \sigma$ and $\underline{\beta}$ obey the same scaling as in (1.16), while each $\nabla_{4}$ derivative costs an additional power of $\delta$ :

$$
\begin{array}{r}
\delta\|\nabla \alpha\|_{L^{2}\left(H_{0}\right)}+\|\nabla \beta\|_{L^{2}\left(H_{0}\right)}+\delta^{-1 / 2}\|\nabla(\varrho, \sigma)\|_{L^{2}\left(H_{0}\right)}+\delta^{-3 / 2}\|\nabla \underline{\beta}\|_{L^{2}\left(H_{0}\right)}<\infty, \\
\delta^{2}\left\|\nabla_{4} \alpha\right\|_{L^{2}\left(H_{0}\right)}+\delta\left\|\nabla_{4} \beta\right\|_{L^{2}\left(H_{0}\right)}+\delta^{1 / 2}\left\|\nabla_{4}(\varrho, \sigma)\right\|_{L^{2}\left(H_{0}\right)}+\delta^{-1 / 2}\left\|\nabla_{4} \underline{\beta}\right\|_{L^{2}\left(H_{0}\right)}<\infty .
\end{array}
$$

Moreover one can derive estimates for the Ricci coefficients, in various norms, weighted by appropriated powers of $\delta$. Note that if one were to neglect the quadratic terms in (1.7), then the expected scaling behavior in $\delta$ would have been

$$
\delta\|\alpha\|_{L^{2}\left(H_{0}\right)}+\|\beta\|_{L^{2}\left(H_{0}\right)}+\delta^{-1}\|(\varrho, \sigma)\|_{L^{2}\left(H_{0}\right)}+\delta^{-2}\|\underline{\beta}\|_{L^{2}\left(H_{0}\right)}<\infty .
$$

Most of the body of work in $[\mathrm{C}]$ is to prove that these estimates can be propagated in the entire space-time region $\mathcal{D}\left(u_{*}, \delta\right)$, with $u_{*}$ of size 1 and $\delta$ sufficiently small, and thus fulfill the necessary conditions for the formation of a trapped surface along the lines of the heuristic argument presented above. The proof of such estimates, which follows the main outline of the proof of stability of Minkowski space, as in [CK] and [KN], requires a step-by-step analysis to make sure that all estimates are consistent with the assigned powers of $\delta$. This task is made particularly taxing in view of the fact that there are many non-linear interferences which have to be tracked precisely.

\subsection{Outline of Christodoulou's propagation estimates}

To see what this entails it pays to say a few words about the strategy of the proof. As in $[\mathrm{CK}]$ and $[\mathrm{KN}]$ the centerpiece of the entire proof consists of proving space-time curvature estimates consistent with (1.16). In this case however the primary attention has to be given to the stratification of the estimates for different curvature components based on their $\delta$-weights. This is done using the Bianchi identities

$$
D_{[\varepsilon} R_{\alpha \beta] \gamma \delta}=0
$$

the associated Bel-Robinson tensor $Q$ and carefully chosen vector fields $X$ whose deformation tensors ${ }^{(X)} \pi$ depend only on the Ricci coefficients $\chi, \omega, \eta, \underline{\chi}, \underline{\omega}$ and $\underline{\eta}$. These 
vector fields can be used either as commutation vector fields or multipliers. In the latter case we would have

$$
D^{\delta}\left(Q_{\alpha \beta \gamma \delta} X^{\alpha} Y^{\beta} Z^{\delta}\right)=Q\left({ }^{(X)} \pi, Y, Z\right)+\ldots
$$

As multipliers $X, Y$ and $Z$ we can choose the vector fields $e_{3}$ and $e_{4}$. The choice

$$
X=Y=Z=e_{4}
$$

leads, after integration on $\mathcal{D}(u, \underline{u})$, to

$$
\|\alpha\|_{L^{2}\left(H_{u}^{(0, \underline{u})}\right)}^{2}+\|\beta\|_{L^{2}\left(\underline{H}_{\underline{u}}^{(0, u)}\right)}^{2}=\|\alpha\|_{L^{2}\left(H_{0}^{(0, \underline{u})}\right)}^{2}+\iint_{\mathcal{D}(u, \underline{u})} 3 Q\left({ }^{(4)} \pi, e_{4}, e_{4}\right),
$$

where $\pi$ is the deformation tensor of $e_{4}$. Since the initial data at $H_{0}$ satisfies (1.16), we write

$$
\delta^{2}\left(\|\alpha\|_{L^{2}\left(H_{u}^{(0, \underline{u})}\right)}^{2}+\|\beta\|_{L^{2}\left(\underline{H}_{\underline{u}}^{(0, u)}\right)}^{2}\right)=\delta^{2}\|\alpha\|_{L^{2}\left(H_{0}^{(0, \underline{u})}\right)}^{2}+3 \delta^{2} \iint_{\mathcal{D}(u, \underline{u})} Q\left({ }^{(4)} \pi, e_{4}, e_{4}\right)
$$

and expect to bound the double integral term on the right. One can derive similar identities for all other possible choices of $X, Y$ and $Z$ among the set $\left\{e_{3}, e_{4}\right\}$. This allows one to estimate both the $L^{2}(H)$ norms of $\alpha, \beta, \varrho, \sigma$ and $\underline{\beta}$, and the $L^{2}(\underline{H})$ norms of $\beta, \varrho$, $\sigma, \underline{\alpha}$ and $\underline{\beta}$, with appropriate $\delta$-weights, in terms of the corresponding $\delta$-weighted $L^{2}\left(H_{0}\right)$ norms of $\alpha, \beta, \varrho, \sigma$ and $\underline{\beta}$, and space-time integrals of $Q\left({ }^{(4)} \pi, e_{\mu}, e_{\nu}\right)$ and $Q\left({ }^{(3)} \pi, e_{\mu}, e_{\nu}\right)$ with $\mu, \nu=3,4$. We can thus extend the initial estimates (1.16) to every null hypersurface $H_{u}$ in our slab provided we can bound all the double integrals on the right-hand side of our integral identities. Now, both deformation tensors ${ }^{(4)} \pi$ and ${ }^{(3)} \pi$ can be expressed in terms of our connection coefficients $\chi, \omega, \eta, \underline{\chi}, \underline{\omega}$ and $\underline{\eta}$. Since $Q$ is quadratic in $R$, to be able to close estimates for our null curvature components we need to derive sup-norm estimates for all our Ricci coefficients. This leads us to the second pillar of the construction which is to derive estimates for Ricci coefficients in terms of the null curvature components, with the help of the null structure equations (1.6), see also §3.1. Combining these equations with the constrained equations, on fixed 2-surfaces $S(u, \underline{u})$ (see (3.2)) and the null Bianchi identities (see (3.7)), we are lead to precise $\delta$-weighted estimates of all Ricci coefficients in terms of $\delta$-weighted $L^{2}(H)$ and $L^{2}(\underline{H})$ norms of all null curvature components and their derivatives. Thus, in a first approximation, the error terms in the above integral identities are quadratic in $R$ and linear in the deformation tensors ${ }^{(4)} \pi$ and ${ }^{(3)} \pi$, which depend, indirectly, on $R$ and its first derivatives. Therefore, to be able to close, one needs to do the following:

(1) Derive higher-derivative estimates for the curvature components;

(2) Make sure that all error terms can be controlled in terms of the principal terms, in the corresponding energy inequality, or terms which have already been estimated at previous steps. 
Note that (2) here seems counterintuitive in view of the large data character of the problem under consideration. Indeed, typically, in such situations one cannot expect to control the non-linear error terms by the principal energy terms. The miracle here is that the error terms are either linear (in the main energy terms), or they contain factors which have been already estimated in previous steps, or are truly non-linear, in which case they are small in powers of $\delta$ relative to the principal energy terms. This is due to the structure of the error terms, reminiscent of the null condition, in which the factors combine in such a way that the total weight in powers of $\delta$ is positive.

In his work, Christodoulou derives estimates for the first two derivatives of the curvature tensor by commuting the Bianchi identities with the vector fields $L$ and $S=\frac{1}{2}\left(u e_{3}+\underline{u} e_{4}\right)$, and rotation vector fields $O$. This process leads to a proliferation of error terms. Moreover not all error terms which are generated in this way satisfy the following essential requirement, alluded above: that they lead to an overall factor of $\delta^{c}$, with a positive exponent $c$, and thus can be absorbed on the left, for sufficiently small $\delta$. Due to non-linear interactions, Christodoulou has to tackle anomalous error terms which are $O(1)$ in $\delta$. Yet he is able to show, by a careful step-by-step analysis, that all such terms are, indeed, linear relative to terms which have already been estimated and thus only quadratic (i.e. linear in the principal energy norm) relative to the remaining components. They can therefore be absorbed by a standard Grönwall inequality. A similar phenomenon helps him to estimate, step by step, all Ricci coefficients.

\subsection{New initial conditions}

As explained above, the main purpose of this paper is to embed the short-pulse ansatz of Christodoulou into a more general set of initial conditions, based on a different underlying scaling. The new scaling, which we incorporate into our basic norms, allows us to conceptualize the separation between the linear and non-linear terms in the null Bianchi and null structure equations and explain the favorable appearance of additional positive powers of $\delta$ in the non-linear error terms mentioned above. Though the initial conditions required to include Christodoulou's data do not quite satisfy this scaling, the generated anomalies are fewer and thus much easier to track.

We start by observing that a natural alternative to (1.14) which comes to mind, related to the familiar parabolic scaling on null hyperplanes in Minkowski space, is

$$
\widehat{\chi}_{0}(\underline{u}, \omega)=\delta^{-1 / 2} f_{0}\left(\delta^{-1} \underline{u}, \delta^{-1 / 2} \omega\right) \text {. }
$$

This does not quite make sense in our framework of compact 2-surfaces $S(u, \underline{u})$, unless of course one is willing to consider the initial data $\widehat{\chi}_{0}(\underline{u}, \omega)$ supported in the angular sector 
$\omega$ of size $\delta^{1 / 2}$. Such a support assumption would however be in contradiction with the lower bound in (1.12) required to be satisfied for each $\omega \in \mathbb{S}^{2}$.

The following interpretation of (1.20) (compare with (1.15)) makes sense however:

$$
\delta^{k+m / 2} \sup _{0 \leqslant \underline{u} \leqslant \delta}\left\|\nabla_{4}^{k} \nabla^{m} \widehat{\chi}_{0}\right\|_{L^{2}(0, \underline{u})}<\infty, \quad 0 \leqslant k+m \leqslant N
$$

Just as in the derivation of (1.16), we can use the null structure equations (1.6) and the null Bianchi equations (1.7) to derive, from (1.21), that

$$
\begin{array}{r}
\delta^{1 / 2}\|\alpha\|_{L^{2}\left(H_{0}\right)}+\|\beta\|_{L^{2}\left(H_{0}\right)}+\delta^{-1 / 2}\|(\varrho, \sigma)\|_{L^{2}\left(H_{0}\right)}+\delta^{-1}\|\underline{\beta}\|_{L^{2}\left(H_{0}\right)}<\infty, \\
\delta\|\nabla \alpha\|_{L^{2}\left(H_{0}\right)}+\delta^{1 / 2}\|\nabla \beta\|_{L^{2}\left(H_{0}\right)}+\|\nabla(\varrho, \sigma)\|_{L^{2}\left(H_{0}\right)}+\delta^{-1 / 2}\|\nabla \underline{\beta}\|_{L^{2}\left(H_{0}\right)}<\infty, \\
\delta^{3 / 2}\left\|\nabla_{4} \alpha\right\|_{L^{2}\left(H_{0}\right)}+\delta\left\|\nabla_{4} \beta\right\|_{L^{2}\left(H_{0}\right)}+\delta^{1 / 2}\left\|\nabla_{4}(\varrho, \sigma)\right\|_{L^{2}\left(H_{0}\right)}+\left\|\nabla_{4} \underline{\beta}\right\|_{L^{2}\left(H_{0}\right)}<\infty .
\end{array}
$$

We refer to these conditions, consistent with the null parabolic scaling, as $\delta$-coherent assumptions. Note that, unlike in Christodoulou's case, each $\nabla$ derivative costs $\delta^{-1 / 2}$. It turns out that proving the propagation of such estimates can be done easily and systematically without the need of the step-by-step procedure mentioned earlier. In fact one can show, in this case, that all error terms generated in the process of the energy estimates are either quadratic in the curvature and can be easily taken care by Grönwall's inequality or, if cubic, they must come with a factor of $\delta^{1 / 2}$ and therefore can all be absorbed for small values of $\delta$.

The main problem with the ansatz (1.20), as with the initial conditions (1.21), however, is that it is inconsistent with the formation of trapped surface requirements discussed above. One can only hope to show that the expansion scalar $\operatorname{tr} \chi$ along $H_{u}$, at $S(u, \underline{u})$, for some $u \approx 1$, will become negative $\left({ }^{2}\right)$ in a small angular sector of size $\delta^{1 / 2}$. This is because, consistent with (1.22), condition (1.12) may only be satisfied in such a sector.

At this point we abandon the ansatz formulation of the characteristic initial data problem for the Einstein-vacuum equations and replace with a hierarchy of bounds, which "interpolate" between the regular $\delta$-coherent assumptions (1.22) and the estimates (1.16)-(1.17) following from Christodoulou's short-pulse ansatz.

At the level of curvature, the new assumptions correspond to

$$
\begin{array}{r}
\delta\|\alpha\|_{L^{2}\left(H_{0}\right)}+\|\beta\|_{L^{2}\left(H_{0}\right)}+\delta^{-1 / 2}\|(\varrho, \sigma)\|_{L^{2}\left(H_{0}\right)}+\delta^{-1} \| \underline{\beta}_{L^{2}\left(H_{0}\right)}<\infty, \\
\delta\|\nabla \alpha\|_{L^{2}\left(H_{0}\right)}+\delta^{1 / 2}\|\nabla \beta\|_{L^{2}\left(H_{0}\right)}+\|\nabla(\varrho, \sigma)\|_{L^{2}\left(H_{0}\right)}+\delta^{-1 / 2}\|\nabla \underline{\beta}\|_{L^{2}\left(H_{0}\right)}<\infty, \\
\delta^{2}\left\|\nabla_{4} \alpha\right\|_{L^{2}\left(H_{0}\right)}+\delta\left\|\nabla_{4} \beta\right\|_{L^{2}\left(H_{0}\right)}+\delta^{1 / 2}\left\|\left(\nabla_{4} \varrho, \nabla_{4} \sigma\right)\right\|_{L^{2}\left(H_{0}\right)}+\left\|\nabla_{4} \underline{\beta}\right\|_{L^{2}\left(H_{0}\right)}<\infty .
\end{array}
$$

$\left({ }^{2}\right)$ We could call such a region locally trapped, or a pre-scar. 
Observe that, by comparison with (1.22), the only anomalous terms are $\|\alpha\|_{L^{2}\left(H_{0}\right)}$ and $\left\|\nabla_{4} \alpha\right\|_{L^{2}\left(H_{0}\right)}$.

In the next section we make our initial data assumptions precise, state the main results and explain the strategy of the proof. We close the discussion here with a summary of our approach:

(1) Replace the short-pulse ansatz of Christodoulou with a larger class of data satisfying (1.23).

(2) Prove propagation of the curvature estimates consistent with (1.23) through the domain of existence and show that these (weaker) estimates are sufficient for the existence result.

(3) The propagation estimates involve only the $L^{2}$ based norms of curvature and its first derivatives, but generate non-linear terms involving both the Ricci coefficients and its first derivatives. To close such estimates requires addressing two major difficulties:

- Regularity problem: show that the $L^{2}$ propagation curvature estimates are sufficient to control the Ricci coefficients (in $L^{\infty}$ ) and its first and even second derivatives in appropriate norms required by the non-linear terms in the curvature estimates

- $\delta$-consistency problem: show that the non-linear terms are either effectively linear (in curvature and its derivatives), and thus can be handled by the Grönwall inequality, or contain a smallness coefficient generated by an additional power of the parameter $\delta$. Our approach, based on the weaker propagation estimates (1.23), is particularly suitable for dealing with this problem in that (a) it generates fewer borderline terms of the first kind, and (b) it naturally lends itself to the introduction of a notion of scale-invariant norms relative to which the structure of the non-linear terms and their $\delta$-smallness become apparent and nearly universal.

(4) The propagation estimates consistent with (1.23), and the corresponding Riccicoefficient estimates which they generate, are not strong enough to prove the formation of a trapped surface. However, once such estimates have been proved in the entire domain $\mathcal{D}(u \approx 1, \underline{u}=\delta)$, it is straightforward to impose slightly stronger conditions on the initial data and show that they lead to space-times which satisfy all the necessary conditions to implement, rigorously, the informal argument presented above.

\section{Main results}

\subsection{Initial data assumptions}

We define the initial data quantity

$$
\mathcal{I}^{(0)}=\sup _{0 \leqslant \underline{u} \leqslant \delta} \mathcal{I}^{(0)}(\underline{u}),
$$


where, with the notation convention in (1.15),

$$
\begin{aligned}
\mathcal{I}^{(0)}(\underline{u})=\delta^{1 / 2} & \left\|\widehat{\chi}_{0}\right\|_{L^{\infty}(0, \underline{u})}+\sum_{k=0}^{2} \delta^{1 / 2}\left\|\left(\delta \nabla_{4}\right)^{k} \widehat{\chi}_{0}\right\|_{L^{2}(0, \underline{u})} \\
& +\sum_{k=0}^{1} \sum_{m=1}^{4} \delta^{1 / 2}\left\|\left(\delta^{1 / 2} \nabla\right)^{m-1}\left(\delta \nabla_{4}\right)^{k} \nabla \widehat{\chi}_{0}\right\|_{L^{2}(0, \underline{u})} .
\end{aligned}
$$

Our main assumption, replacing Christodoulou's ansatz, is

$$
\mathcal{I}^{(0)}<\infty
$$

We show that, under this assumption and for sufficiently small $\delta>0$, the space-time slab $\mathcal{D}(u, \delta)$ can be extended for values of $u \geqslant 1$, with precise estimates for all Ricci coefficients of the double null foliation and null components of the curvature tensor. We can then show, by a slight modification of this assumption together with Christodoulou's lower bound assumption on $\int_{0}^{\delta}\left|\widehat{\chi}_{0}\right|^{2} d u^{\prime}$ (see equations (14) and (15) in [C]), that a trapped surface must form in $\mathcal{D}(u \approx 1, \delta)$. As in the case of $[\mathrm{C}]$, most of the work is required to prove the semi-global result concerning the double null foliation. Once this is established, the actual formation of trapped surfaces is proved by making a slight modification of the main assumption (2.2) and following the heuristic argument outlined before. In addition, we show that a small modification of the regular $\delta$-coherence assumption leads to the formation of a pre-scar.

\subsection{Curvature norms}

To give a precise formulation of our result we need to introduce the following norms:

$$
\begin{aligned}
& \mathcal{R}_{0}(u, \underline{u}):=\delta\|\alpha\|_{H_{u}^{(0, \underline{u})}}+\|\beta\|_{H_{u}^{(0, \underline{u})}}+\delta^{-1 / 2}\|(\varrho, \sigma)\|_{H_{u}^{(0, \underline{u})}}+\delta^{-1}\|\underline{\beta}\|_{H_{u}^{(0, \underline{u})}}, \\
& \mathcal{R}_{1}(u, \underline{u}):=\delta\|\nabla \alpha\|_{H_{u}^{(0, \underline{u})}}+\delta^{1 / 2}\|\nabla \beta\|_{H_{u}^{(0, \underline{u})}}+\|\nabla(\varrho, \sigma)\|_{H_{u}^{(0, \underline{u})}}+\delta^{-1 / 2}\|\nabla \underline{\beta}\|_{H_{u}^{(0, \underline{u})}} \\
& +\delta\left\|\nabla_{4} \alpha\right\|_{H_{u}^{(0, \underline{u})}}, \\
& \underline{\mathcal{R}}_{0}(u, \underline{u}):=\delta\|\beta\|_{\underline{H}_{\underline{u}}^{(0, u)}}+\|(\varrho, \sigma)\|_{\underline{H}_{\underline{u}}^{(0, u)}}+\delta^{-1 / 2}\|\underline{\beta}\|_{\underline{H}_{\underline{u}}^{(0, u)}}+\delta^{-1}\|\underline{\alpha}\|_{\underline{H}_{\underline{u}}^{(0, u)}}, \\
& \underline{\mathcal{R}}_{1}(u, \underline{u}):=\delta\|\nabla \beta\|_{\underline{H}_{\underline{u}}^{(0, u)}}+\delta^{1 / 2}\|\nabla(\varrho, \sigma)\|_{\underline{H}_{\underline{u}}^{(0, u)}}+\|\nabla \underline{\beta}\|_{\underline{H}_{\underline{u}}^{(0, u)}}+\delta^{-1 / 2}\|\nabla \underline{\alpha}\|_{\underline{H}_{\underline{u}}^{(0, u)}} \\
& +\delta^{-1}\left\|\nabla_{3} \underline{\alpha}\right\|_{\underline{H}}^{(0, u)} \text {. }
\end{aligned}
$$

We also set $\mathcal{R}_{0}$ and $\mathcal{R}_{1}$ to be the supremum over $u$ and $\underline{u}$ in our space-time slab of $\mathcal{R}_{0}(u, \underline{u})$ and $\mathcal{R}_{1}(u, \underline{u})$, respectively, and similarly for the norms $\underline{\mathcal{R}}$. Also we write $\mathcal{R}=\mathcal{R}_{0}+\mathcal{R}_{1}$ and $\underline{\mathcal{R}}=\underline{\mathcal{R}}_{0}+\underline{\mathcal{R}}_{1}$. Finally, $\mathcal{R}^{(0)}$ denotes the initial value for the norm $\mathcal{R}$, i.e.

$$
\mathcal{R}^{(0)}=\sup _{0 \leqslant \underline{u} \leqslant \delta}\left(\mathcal{R}_{0}(0, \underline{u})+\mathcal{R}_{1}(0, \underline{u})\right)=\mathcal{R}_{0}(0, \delta)+\mathcal{R}_{1}(0, \delta) .
$$


Note that the only $\nabla_{4}$ derivative appearing in the norms above is that of $\alpha$. All other $\nabla_{4}$ derivatives can be deduced from the null Bianchi equations and thus do not need to be incorporated in our norms. We denote the norms of a specific curvature component $\psi$ by $\mathcal{R}_{0}[\psi]$ and $\mathcal{R}_{1}[\psi]$.

\subsection{Ricci-coefficient norms}

We introduce norms for the Ricci coefficients $\widehat{\chi}, \operatorname{tr} \chi, \omega, \eta, \underline{\hat{\chi}}, \underline{\omega}, \underline{\eta}$ and $\widetilde{\operatorname{tr}} \underline{\chi}=\operatorname{tr} \underline{\chi}-\operatorname{tr} \underline{\chi}_{0}$, with $\operatorname{tr} \underline{\chi}_{0}=-4 /\left(\underline{u}-u+2 r_{0}\right)$, the flat value of $\operatorname{tr} \underline{\chi}$ along the initial hypersurface $\underline{H}_{0}$.

For any $S=S(u, \underline{u})$ we introduce following norms ${ }^{(S)} \mathcal{O}_{s, p}(u, \underline{u})$ :

$$
\begin{aligned}
& { }^{(S)} \mathcal{O}_{0, \infty}(u, \underline{u})=\delta^{1 / 2}\left(\|\chi\|_{L^{\infty}(S)}+\|\omega\|_{L^{\infty}(S)}\right)+\|\eta\|_{L^{\infty}(S)}+\|\underline{\eta}\|_{L^{\infty}(S)} \\
& +\delta^{-1 / 2}\left(\|\underline{\hat{\chi}}\|_{L^{\infty}(S)}+\|\widetilde{\operatorname{tr} \underline{\chi}}\|_{L^{\infty}(S)}+\|\underline{\omega}\|_{L^{\infty}(S)}\right), \\
& { }^{(S)} \mathcal{O}_{0,4}(u, \underline{u})=\delta^{1 / 2}\|\chi\|_{L^{4}(S)}+\delta^{1 / 4}\|\omega\|_{L^{4}(S)}+\delta^{-1 / 4}\left(\|\eta\|_{L^{4}(S)}+\|\underline{\eta}\|_{L^{4}(S)}\right) \\
& +\delta^{-1 / 2}\|\underline{\hat{\chi}}\|_{L^{4}(S)}+\delta^{-3 / 4}\left(\|\widetilde{\operatorname{tr} \underline{\chi}}\|_{L^{4}(S)}+\|\underline{\omega}\|_{L^{4}(S)}\right), \\
& { }^{(S)} \mathcal{O}_{1,4}(u, \underline{u})=\delta^{3 / 4}\left(\|\nabla \chi\|_{L^{4}(S)}+\|\nabla \omega\|_{L^{4}(S)}\right)+\delta^{1 / 4}\left(\|\nabla \eta\|_{L^{4}(S)}+\|\nabla \underline{\eta}\|_{L^{4}(S)}\right) \\
& +\delta^{-1 / 4}\left(\|\nabla \underline{\chi}\|_{L^{4}(S)}+\|\nabla \underline{\omega}\|_{L^{4}(S)}\right), \\
& { }^{(S)} \mathcal{O}_{1,2}(u, \underline{u})=\delta^{1 / 2}\left(\|\nabla \chi\|_{L^{2}(S)}+\|\nabla \omega\|_{L^{2}(S)}\right)+\|\nabla \eta\|_{L^{2}(S)}+\|\nabla \underline{\eta}\|_{L^{2}(S)} \\
& +\delta^{-1 / 2}\left(\|\nabla \underline{\chi}\|_{L^{2}(S)}+\|\nabla \underline{\omega}\|_{L^{2}(S)}\right) .
\end{aligned}
$$

Also,

$$
\begin{aligned}
{ }^{(H)} \mathcal{O}(u, \underline{u})=\delta^{1 / 2}\left(\left\|\nabla^{2} \chi\right\|_{L^{2}\left(H_{u}^{(0, \underline{u})}\right)}+\left\|\nabla^{2} \omega\right\|_{L^{2}\left(H_{u}^{(0, \underline{u})}\right)}\right) \\
+\left(\left\|\nabla^{2} \eta\right\|_{L^{2}\left(H_{u}^{(0, \underline{u})}\right)}+\left\|\nabla^{2} \underline{\eta}\right\|_{L^{2}\left(H_{u}^{(0, \underline{u})}\right)}\right) \\
+\delta^{-1 / 2}\left(\left\|\nabla^{2} \underline{\chi}\right\|_{L^{2}\left(H_{u}^{(0, \underline{u})}\right)}+\left\|\nabla^{2} \underline{\omega}\right\|_{L^{2}\left(H_{u}^{(0, \underline{u})}\right)}\right)
\end{aligned}
$$

and

$$
\begin{aligned}
(\underline{H}) \mathcal{O}(u, \underline{u})=\delta^{1 / 2} & \left(\left\|\nabla^{2} \chi\right\|_{L^{2}\left(\underline{H}_{\underline{u}}^{(0, u)}\right)}+\left\|\nabla^{2} \omega\right\|_{L^{2}\left(\underline{H}_{\underline{u}}^{(0, u)}\right)}\right) \\
& +\left(\left\|\nabla^{2} \eta\right\|_{L^{2}\left(\underline{H}_{\underline{u}}^{(0, u)}\right)}+\left\|\nabla^{2} \underline{\eta}\right\|_{L^{2}\left(\underline{H}_{\underline{u}}^{(0, u)}\right)}\right) \\
& +\delta^{-1 / 2}\left(\left\|\nabla^{2} \underline{\chi}\right\|_{L^{2}\left(\underline{H}_{\underline{u}}^{(0, u)}\right)}+\left\|\nabla^{2} \underline{\omega}\right\|_{L^{2}\left(\underline{H}_{\underline{H}}^{(0, u)}\right)}\right) .
\end{aligned}
$$

We define the norms ${ }^{(S)} \mathcal{O}_{0,4},{ }^{(S)} \mathcal{O}_{0, \infty},{ }^{(S)} \mathcal{O}_{1,2},{ }^{(S)} \mathcal{O}_{1,4},{ }^{(H)} \mathcal{O}$ and ${ }^{(H)} \mathcal{O}$ to be the supremum over all values of $u$ and $\underline{u}$ in our slab of the corresponding norms. Finally we set the total Ricci norm to be

$$
\mathcal{O}={ }^{(S)} \mathcal{O}_{0, \infty}+{ }^{(S)} \mathcal{O}_{0,4}+{ }^{(S)} \mathcal{O}_{1,2}+{ }^{(S)} \mathcal{O}_{1,4}+{ }^{(H)} \mathcal{O}+{ }^{(\underline{H})} \mathcal{O}
$$


and denote by $\mathcal{O}^{(0)}$ the corresponding norm of the initial hypersurface $H_{0}$. We further differentiate between the first-order norms, $\mathcal{O}_{[1]}={ }^{(S)} \mathcal{O}_{0,4}+{ }^{(S)} \mathcal{O}_{1,2}$, and second-order ones, $\mathcal{O}_{[2]}={ }^{(S)} \mathcal{O}_{1,4}+{ }^{(H)} \mathcal{O}+{ }^{(H)} \mathcal{O}$.

\subsection{Main theorems}

We are now ready to state our main result. The first result follows from analyzing assumption (2.1) on the initial hypersurface $H_{0}$.

Proposition 2.1. In view of our initial assumption (2.2) we have, for sufficiently small $\delta>0$, along $H_{0}$,

$$
\mathcal{R}^{(0)}+\mathcal{O}^{(0)} \lesssim \mathcal{I}^{(0)}
$$

The proof of the proposition follows by analyzing the null structure and null Bianchi equations restricted to the initial hypersurface $H_{0}$, as in [C, Chapter 2]. In view of this result we may replace assumption (2.1) with (2.5), as an initial data assumption. Alternatively we may assume only that $\mathcal{R}^{(0)} \lesssim \mathcal{I}^{(0)}$. It is not too hard to see, following roughly the same steps as in the proof of Proposition 2.1, that, for small $\delta$, we would also have $\mathcal{O}^{(0)} \lesssim \mathcal{I}^{(0)}$.

THEOREM 2.2. (Main theorem) Assume that $\mathcal{R}^{(0)} \lesssim \mathcal{I}^{(0)}$ for an arbitrary constant $\mathcal{I}^{(0)}$. Then, there exists a sufficiently small $\delta>0$ such that

$$
\mathcal{R}+\underline{\mathcal{R}}+\mathcal{O} \lesssim \mathcal{I}^{(0)}
$$

THEOREM 2.3. Assume that, in addition to (2.1), we also have, for $2 \leqslant k \leqslant 4$,

$$
\left\|\left(\delta^{1 / 2} \nabla\right)^{k} \widehat{\chi}_{0}\right\|_{L^{2}(0, \underline{u})} \leqslant \varepsilon
$$

for a sufficiently small parameter $\varepsilon$ with $0<\delta \ll \varepsilon$. Assume also that $\hat{\chi}_{0}$ satisfies $(1.12)$. Then, for $\delta>0$ sufficiently small, a trapped surface must form in the slab $\mathcal{D}(u \approx 1, \delta)$.

Proof. We sketch below the proof of Theorem 2.3.

Step 1. We reinterpret (2.7) in terms of the curvature norms according to the following result.

Proposition 2.4. Under the smallness condition (2.7), the initial curvature norms satisfy, in addition to the estimates of Proposition 2.1,

$$
\delta^{1 / 2}\|\nabla \beta\|_{H_{0}^{(0, \delta)}}+\|\nabla(\varrho, \sigma)\|_{H_{0}^{(0, \delta)}}+\delta^{-1 / 2}\|\nabla \underline{\beta}\|_{H_{0}^{(0, \delta)}} \leqslant \varepsilon
$$


The proof is standard and will be omitted.

Step 2. We show, see the end of $\S 15$, that this condition can be propagated in the entire slab $\mathcal{D}(u \approx 1, \delta)$.

Proposition 2.5. Under the assumptions (2.7) we have, uniformly in $u \lesssim 1$ and $\underline{u} \leqslant \delta$, for $\delta$ sufficiently small,

$$
\begin{aligned}
& \delta^{1 / 2}\|\nabla \beta\|_{H_{u}^{(0, \underline{u})}}+\|\nabla(\varrho, \sigma)\|_{H_{u}^{(0, \underline{u})}}+\delta^{-1 / 2}\|\nabla \underline{\beta}\|_{H_{u}^{(0, \underline{u})}} \leqslant \varepsilon, \\
& \delta^{1 / 2}\|\nabla(\varrho, \sigma)\|_{\underline{H}_{\underline{u}}^{(0, u)}}+\|\nabla \underline{\beta}\|_{\underline{H}_{\underline{u}}^{(0, u)}}+\delta^{-1 / 2}\left\|\nabla \underline{\alpha}_{\underline{\underline{H}}}\right\|_{\underline{u}}^{(0, u)} \leqslant \varepsilon .
\end{aligned}
$$

Step 3. We return to the system (1.8)-(1.9),

$$
\begin{aligned}
\nabla_{4} \operatorname{tr} \chi+\frac{1}{2}(\operatorname{tr} \chi)^{2} & =-|\widehat{\chi}|^{2}-2 \omega \operatorname{tr} \chi, \\
\nabla_{3} \widehat{\chi}+\frac{1}{2}(\operatorname{tr} \underline{\chi}) \hat{\chi} & =\nabla \widehat{\otimes} \eta+2 \underline{\omega} \widehat{\chi}-\frac{1}{2}(\operatorname{tr} \chi) \underline{\hat{\chi}}+\eta \widehat{\otimes} \eta,
\end{aligned}
$$

responsible, as we have seen, for the formation of a trapped surface. Theorem 2.2 implies that the terms ignored in our heuristic derivation are negligible. Specifically, the bounds $|\omega \operatorname{tr} \chi| \lesssim \delta^{-1 / 2}$ and $|\underline{\omega} \hat{\chi}|+|(\operatorname{tr} \chi) \underline{\chi}|+|\eta \widehat{\otimes} \eta| \lesssim 1$ should be compared to the principle terms of size $\delta^{-1}$ and $\delta^{-1 / 2}$ in the first and second equation, respectively. We can also easily verify the other bounds in (1.13) with the exception of that for $\nabla \widehat{\otimes} \eta$. The additional condition (2.7) is imposed in fact precisely in order to assure that the linear term $\nabla \widehat{\otimes} \eta$ in (1.9) is sufficiently small. To control this term we rely on the following proposition.

Proposition 2.6. Under the assumptions of Theorem 2.3, the solution ${ }^{(3)} \phi$ of the problem $\nabla_{3}^{(3)} \phi=\nabla \widehat{\otimes} \eta$, with trivial initial data on $H_{0}$, satisfies

$$
\left.\right|^{(3)} \phi \mid \leqslant C \delta^{-1 / 2} \varepsilon^{1 / 4}
$$

The proof of Proposition 2.6, which appears in $\S 15.6$, depends on the arguments of $\S 11$, in particular Proposition 11.8. The argument for the formation of a trapped surface then proceeds as above with a renormalized quantity $\widehat{\chi}^{-{ }^{(3)} \phi}$ in place of $\widehat{\chi}$. Note that in view of the estimate on ${ }^{(3)} \phi$, the size of $\widehat{\chi}^{-}{ }^{(3)} \phi$ is comparable to that of $\widehat{\chi}$. An important comment in this regard is that our curvature propagation estimates do not allow us to control the $L^{\infty}$ norm of $\nabla \widehat{\otimes} \eta$, let alone prove the bound stated in (1.13). This regularity problem, which is discussed in the two remarks below, is resolved with the help of the renormalized estimates for the Ricci coefficients in $\S 11$, of which Proposition 2.6 is an important example.

Remark 2.7. We remark that, while a loss of derivative occurs when passing from assumption $(2.2)$ to assumption $\mathcal{R}^{(0)} \lesssim \mathcal{I}^{(0)}$ in the main theorem, no further derivative losses occur in (2.6). 
Remark 2.8. By contrast with [C], where two derivatives of the curvature and up to three derivatives of the Ricci coefficients are needed, here we only need one derivative of the curvature and two of the Ricci coefficients. This is due to our new refined estimates for the deformation tensor of the angular momentum vector fields $O$. As mentioned above, these vector fields are needed to derive estimates for the angular derivatives of the null curvature components. These new estimates for the deformation tensor of the angular momentum vector fields $O$ are based on the renormalized estimates for the Ricci coefficients developed in $\S 11$. Together with the trace estimates for the curvature components, which serve as a replacement for the failed $H^{1}(S) \subset L^{\infty}(S)$ embedding on a 2 -dimensional surface $S$, proved in $\S 12$, they allow us to limit the degree of differentiability required in the proof to the $L^{2}$ norms of curvature and its first derivatives. Similar ideas related to the gain of differentiability via renormalization and trace estimates were exploited in our earlier work [KR1].

Our next and final result concerns the formation of a pre-scar in an angular sector of size $\delta^{1 / 2}$.

THEOREM 2.9. Let $\varepsilon$ be a small parameter with $0<\delta \ll \varepsilon$. Assume that the initial data $\widehat{\chi}_{0}$ satisfies

$$
\delta^{1 / 2}\left\|\widehat{\chi}_{0}\right\|_{L^{\infty}}+\sum_{k=0}^{1} \sum_{m=0}^{4} \varepsilon\left\|\left(\varepsilon^{-1} \delta^{1 / 2} \nabla\right)^{m}\left(\delta \nabla_{4}\right)^{k} \widehat{\chi}_{0}\right\|_{L^{2}(0, \underline{u})}<\infty
$$

and that the lower bound in (1.12) is satisfied in an angular sector $\omega \in \Lambda$ of size $\delta^{1 / 2}$. Then, for $\delta>0$ sufficiently small, a pre-scar must form in the slab $\mathcal{D}(u \approx 1, \delta)$, i.e. the expansion scalar $\operatorname{tr} \chi(u, \underline{u}, \omega)$ becomes stricly negative for some values of $u \approx 1, \underline{u}=\delta$ and all $\omega \in \Lambda$.

Remark. Theorem 2.9 corresponds to the initial data consistent with the ansatz

$$
\widehat{\chi}_{0}(\underline{u}, \omega)=\delta^{-1 / 2} f_{0}\left(\delta^{-1} \underline{u}, \delta^{-1 / 2} \varepsilon \omega\right)
$$

and localized in an angular sector of size $\delta^{1 / 2} \varepsilon^{-1}$. This should be compared with the data discussed in (1.20). As in Theorem 2.3, additional smallness provided by the parameter $\varepsilon$ is only needed to guarantee the formation of a pre-scar but not required for the proof of the existence result. A direct comparison shows that the data of Theorem 2.9 is significantly more regular than that of Theorems 2.2 and 2.3. In particular, it essentially corresponds to the $\delta$-coherent assumptions, consistent with the natural null parabolic scaling discussed in (1.22). Thus the proof of Theorem 2.9 is significantly easier than that of our main result and will be omitted. 


\subsection{Strategy of the proof}

We divide the proof of the main theorem into three parts. In the first part we derive estimates for the Ricci-coefficient norms $\mathcal{O}$ in terms of the initial data $\mathcal{I}^{(0)}$ and the curvature norms $\mathcal{R}$. More precisely we prove the following result.

Theorem A. Assume that $\mathcal{O}^{(0)}<\infty$ and $\mathcal{R}<\infty$. There is a constant $C$ depending only on $\mathcal{O}^{(0)}, \mathcal{R}$ and $\underline{\mathcal{R}}$ such that

$$
\mathcal{O} \lesssim C\left(\mathcal{O}^{(0)}, \mathcal{R}, \underline{\mathcal{R}}\right)
$$

Moreover,

$$
{ }^{(S)} \mathcal{O}_{0,4}[\underline{\hat{\chi}}] \lesssim \mathcal{O}^{(0)}+C\left(\mathcal{I}^{(0)}, \mathcal{R}, \underline{\mathcal{R}}\right) \delta^{1 / 4} .
$$

We prove the theorem by a bootstrap argument. We start by assuming that there exists a sufficiently large constant $\Delta_{0}$ such that

$$
{ }^{(S)} \mathcal{O}_{0, \infty} \leqslant \Delta_{0}
$$

Based on this assumption we show that, if $\delta$ is sufficiently small, estimate (2.11) also holds. This allows us to derive a better estimate than (2.13).

In the second part we need to define angular momentum operators $O$ and show that their deformation tensors satisfy compatible estimates, stated in Theorem B, at the end of $\S 13$.

Finally in the last and main part we need to use the estimates of Theorems A and $\mathrm{B}$ to derive estimates for the curvature norms $\mathcal{R}$ and thus end the proof of the main theorem.

Theorem C. There exists $\delta$ sufficiently small such that

$$
\mathcal{R}+\underline{\mathcal{R}} \lesssim \mathcal{I}_{0}
$$

Theorem $\mathrm{C}$ is proved in $\S 14$ and $\S 15$.

\subsection{Signature and scaling}

Our norms are intimately tied with a natural scaling which we introduce below.

Signature. To every null curvature component $\alpha, \beta, \varrho, \sigma, \underline{\alpha}$ and $\underline{\beta}$, to every null Ricci-coefficient component $\chi, \zeta, \omega, \eta, \underline{\omega}$ and $\underline{\eta}$, and to the metric $\gamma$ we assign a signature according to the following rule:

$$
\operatorname{sgn}(\phi)=1 N_{4}(\phi)+\frac{1}{2} N_{a}(\phi)+0 N_{3}(\phi)-1,
$$


where $N_{4}(\phi), N_{3}(\phi)$ and $N_{a}(\phi), a=1,2$, denote the number of times $e_{4}, e_{3}$ and $e_{a}$, respectively, appear in the definition of $\phi$. Thus,

$$
\operatorname{sgn}(\alpha)=2, \quad \operatorname{sgn}(\beta)=1+\frac{1}{2}, \quad \operatorname{sgn}(\varrho, \sigma)=1, \quad \operatorname{sgn}(\underline{\beta})=\frac{1}{2} \quad \text { and } \quad \operatorname{sgn}(\underline{\alpha})=0 .
$$

Also,

$$
\operatorname{sgn}(\chi)=\operatorname{sgn}(\omega)=1, \quad \operatorname{sgn}(\zeta, \eta, \underline{\eta})=\frac{1}{2} \quad \text { and } \quad \operatorname{sgn}(\underline{\chi})=\operatorname{sgn}(\underline{\omega})=\operatorname{sgn}(\gamma)=0 .
$$

Consistent with this definition we have, for any given null component $\phi$,

$$
\operatorname{sgn}\left(\nabla_{4} \phi\right)=1+\operatorname{sgn}(\phi), \quad \operatorname{sgn}(\nabla \phi)=\frac{1}{2}+\operatorname{sgn}(\phi) \quad \text { and } \quad \operatorname{sgn}\left(\nabla_{3} \phi\right)=\operatorname{sgn}(\phi) .
$$

Also, based on our convention,

$$
\operatorname{sgn}\left(\phi_{1} \phi_{2}\right)=\operatorname{sgn}\left(\phi_{1}\right)+\operatorname{sgn}\left(\phi_{2}\right) .
$$

Remark. All terms in a given null structure or null Bianchi identity (see equations (3.1)-(3.7)) have the same overall signature. Remark also that the definition (2.15) applies only to the null components of the curvature tensor and Ricci coefficients and not to their tensor products (i.e. not to null decompositions of tensor products).

We now introduce a notion of scale for any quantity $\phi$ which has a signature $\operatorname{sgn}(\phi)$, in particular for our basic null curvature quantities $\alpha, \beta, \varrho, \sigma, \underline{\alpha}$ and $\underline{\beta}$, and null Riccicoefficient components $\chi, \zeta, \omega, \eta, \underline{\omega}$ and $\underline{\eta}$. This scaling plays a fundamental role in our work.

Definition 2.10. For an arbitrary horizontal tensor field $\phi$, with a well-defined signature $\operatorname{sgn}(\phi)$, we set

$$
\operatorname{sc}(\phi)=-\operatorname{sgn}(\phi)+\frac{1}{2}
$$

Observe that $\operatorname{sc}\left(\nabla_{L} \phi\right)=\operatorname{sc}(\phi)-1, \operatorname{sc}(\nabla \phi)=\operatorname{sc}(\phi)-\frac{1}{2}$ and $\operatorname{sc}\left(\nabla_{\underline{L}} \phi\right)=\operatorname{sc}(\phi)$. For a given product of two horizontal tensor fields, we have

$$
\operatorname{sc}\left(\phi_{1} \phi_{2}\right)=\operatorname{sc}\left(\phi_{1}\right)+\operatorname{sc}\left(\phi_{2}\right)-\frac{1}{2}
$$

\subsection{Scale-invariant norms}

For any horizontal tensor field $\psi$ with scale $\operatorname{sc}(\psi)$, we define the following scale-invariant norms along the null hypersurfaces $H=H_{u}^{(0, \delta)}$ and $\underline{H}=\underline{H}_{\underline{u}}^{(0,1)}$ :

$$
\|\psi\|_{\mathcal{L}_{(\mathrm{sc})}^{2}(H)}=\delta^{-\operatorname{sc}(\psi)-1}\|\psi\|_{L^{2}(H)} \quad \text { and } \quad\|\psi\|_{\mathcal{L}_{(\mathrm{sc})}^{2}(\underline{H})}=\delta^{-\operatorname{sc}(\psi)-1 / 2}\|\psi\|_{L^{2}(\underline{H})} .
$$


We also define the scale-invariant norms on the 2 -surface $S=S_{u, \underline{u}}$ by

$$
\|\psi\|_{\mathcal{L}_{(\mathrm{sc})}^{p}(S)}=\delta^{-\operatorname{sc}(\psi)-1 / p}\|\psi\|_{L^{p}(S)}
$$

In particular,

$$
\|\psi\|_{\mathcal{L}_{(\mathrm{sc})}^{2}(S)}=\delta^{-\operatorname{sc}(\psi)-1 / 2}\|\psi\|_{L^{2}(S)} \quad \text { and } \quad\|\psi\|_{\mathcal{L}_{(\mathrm{sc})}^{\infty}(S)}=\delta^{-\operatorname{sc}(\psi)}\|\psi\|_{L^{\infty}(S)}
$$

Observe that we have

$$
\|\psi\|_{\mathcal{L}_{(\mathrm{sc})}^{2}\left(H_{u}^{(0, \underline{u})}\right)}^{2}=\delta^{-1} \int_{0}^{\underline{u}}\|\psi\|_{\mathcal{L}_{(\mathrm{sc})}^{2}\left(u, \underline{u}^{\prime}\right)}^{2} d \underline{u}^{\prime} \quad \text { and } \quad\|\psi\|_{\mathcal{L}_{(\mathrm{sc})}^{2}\left(\underline{H}_{\underline{u}}^{(0, u)}\right)}^{2}=\int_{0}^{u}\|\psi\|_{\mathcal{L}_{(\mathrm{sc})}^{2}\left(u^{\prime}, \underline{u}\right)}^{2} d u^{\prime} .
$$

We denote the scale-invariant $L^{\infty}$ norm in $\mathcal{D}$ by $\|\psi\|_{\mathcal{L}_{(\mathrm{sc})}^{\infty}}$.

Remark. Note that the norms above are scale invariant if we take into account the scales of the $L^{2}$ norms along $H$ and $\underline{H}$, given by

$$
\operatorname{sc}\left(\|\cdot\|_{L^{2}\left(H_{u}^{0, \delta}\right)}\right)=1, \quad \operatorname{sc}\left(\|\cdot\|_{L^{2}\left(\underline{H}_{\underline{u}}^{0,1}\right)}\right)=\frac{1}{2} \quad \text { and } \quad \operatorname{sc}\left(\|\cdot\|_{L^{p}(S)}\right)=\frac{1}{p} .
$$

Moreover, they are consistent with the convention

$$
\nabla_{4} \sim \delta^{-1}, \quad \nabla \sim \delta^{-1 / 2} \text { and } \quad \nabla_{3} \sim 1
$$

In view of (2.18), all standard product estimates in the usual $L^{p}$ spaces translate into product estimates in $\mathcal{L}_{(\mathrm{sc})}$ spaces with a gain of $\delta^{1 / 2}$. Thus, for example,

$$
\left\|\psi_{1} \psi_{2}\right\|_{\mathcal{L}_{(\mathrm{sc})}^{2}(S)} \lesssim \delta^{1 / 2}\left\|\psi_{1}\right\|_{\mathcal{L}_{(\mathrm{sc})}^{\infty}(S)}\left\|\psi_{2}\right\|_{\mathcal{L}_{(\mathrm{sc})}^{2}(S)}
$$

and

$$
\left\|\psi_{1} \psi_{2}\right\|_{\mathcal{L}_{(\mathrm{sc})}^{2}(H)} \lesssim \delta^{1 / 2}\left\|\psi_{1}\right\|_{\mathcal{L}_{(\mathrm{sc})}^{\infty}(H)}\left\|\psi_{2}\right\|_{\mathcal{L}_{(\mathrm{sc})}^{2}(H)} .
$$

Remark 2.11. If $f$ is a scalar function constant along the surface $S(u, \underline{u}) \subset \mathcal{D}$, we have

$$
\|f \psi\|_{\mathcal{L}_{(\mathrm{sc})}^{p}(S)} \lesssim\|\psi\|_{\mathcal{L}_{(\mathrm{sc})}^{p}(S)}
$$

or, if $f$ is also bounded on $H$,

$$
\|f \psi\|_{\mathcal{L}_{(\mathrm{sc})}^{2}(H)} \lesssim\|\psi\|_{\mathcal{L}_{(\mathrm{sc})}^{2}(H)}
$$

This remark applies in particular to the constant $\operatorname{tr} \underline{\chi}_{0}=4 /\left(2 r_{0}+\underline{u}-u\right)$. 
We can reinterpret our main curvature and Ricci-coefficient norms in light of the scale-invariant norms. Thus $(2.3)$ can be rewritten in the form $\left({ }^{3}\right)$

$$
\begin{aligned}
& \mathcal{R}_{0}(u, \underline{u}):=\delta^{1 / 2}\|\alpha\|_{\mathcal{L}_{(\mathrm{sc})}^{2}\left(H_{u}^{(0, \underline{u})}\right)}+\|(\beta, \varrho, \sigma, \underline{\beta})\|_{\mathcal{L}_{(\mathrm{sc})}^{2}\left(H_{u}^{(0, \underline{u})}\right)}, \\
& \mathcal{R}_{1}(u, \underline{u}):=\delta^{1 / 2}\left\|\nabla_{4} \alpha\right\|_{\mathcal{L}_{(\mathrm{sc})}^{2}\left(H_{u}^{(0, \underline{u})}\right)}+\|\nabla(\alpha, \beta, \varrho, \sigma, \underline{\beta})\|_{\mathcal{L}_{(\mathrm{sc})}^{2}\left(H_{u}^{(0, \underline{u}))}\right)}, \\
& \underline{\mathcal{R}}_{0}(u, \underline{u}):=\delta^{1 / 2}\|\beta\|_{\mathcal{L}_{(\mathrm{sc})}^{2}\left(\underline{H}_{\underline{u}}^{(u, 0)}\right)}+\|(\varrho, \sigma, \underline{\alpha}, \underline{\beta})\|_{\mathcal{L}_{(\mathrm{sc})}^{2}\left(\underline{H}_{\underline{u}}^{(0, u)}\right)}, \\
& \underline{\mathcal{R}}_{1}(u, \underline{u}):=\left\|\nabla_{3}\right\|_{\mathcal{L}_{(\mathrm{sc})}^{2}\left(\underline{H}_{\underline{u}}^{(u, 0)}\right)}+\|\nabla(\beta, \varrho, \sigma, \underline{\alpha}, \underline{\beta})\|_{\mathcal{L}_{(\mathrm{sc})}^{2}\left(\underline{H}_{\underline{u}}^{(0, u)}\right)}
\end{aligned}
$$

Remark 2.12. All curvature norms are scale invariant except for the anomalous $\|\alpha\|_{\mathcal{L}_{(\mathrm{sc})}^{2}\left(H_{u}^{(0, \underline{u})}\right)},\left\|\nabla_{4} \alpha\right\|_{\mathcal{L}_{(\mathrm{sc})}^{2}\left(H_{u}^{(0, \underline{u})}\right)}$ and $\|\beta\|_{\mathcal{L}_{(\mathrm{sc})}^{2}\left(\underline{H}_{\underline{u}}^{(u, 0)}\right)}$. By abuse of language, in a given context, we refer to $\alpha$, resp. $\beta$, as anomalous.

To rectify the anomaly of $\alpha$ we introduce an additional scale-invariant norm

$$
\mathcal{R}_{0}^{\delta}[\alpha]:=\sup _{{ }^{\delta} H \subset H}\|\alpha\|_{\mathcal{L}_{(\mathrm{sc})}^{2}\left({ }^{\delta} H\right)}
$$

where ${ }^{\delta} H$ is a piece of the hypersurface $H=H_{u}^{0, \delta}$ obtained by evolving a disk $S_{\delta} \subset S_{u, 0}$ of radius $\delta^{1 / 2}$ along the integral curves of the vector field $e_{4}$.

The Ricci-coefficient norms (2.4) can be written as

$$
\begin{aligned}
{ }^{(S)} \mathcal{O}_{0, \infty}(u, \underline{u}) & =\|(\chi, \omega, \eta, \widetilde{\operatorname{tr} \underline{\chi}}, \underline{\hat{\chi}}, \underline{\omega}, \underline{\eta})\|_{\mathcal{L}_{(\mathrm{sc})}^{\infty}(S)}, \\
{ }^{(S)} \mathcal{O}_{0,4}(u, \underline{u}) & =\delta^{1 / 4}\left(\|\widehat{\chi}\|_{\mathcal{L}_{(\mathrm{sc})}^{4}(S)}+\|\underline{\widehat{\chi}}\|_{\mathcal{L}_{(\mathrm{sc})}^{4}(S)}\right)+\|(\operatorname{tr} \chi, \omega, \eta, \widetilde{\operatorname{tr} \underline{\chi}}, \underline{\omega}, \underline{\eta})\|_{\mathcal{L}_{(\mathrm{sc})}^{4}(S)}, \\
{ }^{(S)} \mathcal{O}_{1,4}(u, \underline{u}) & =\|\nabla(\chi, \omega, \eta, \widetilde{\operatorname{tr} \underline{\chi}}, \underline{\hat{\chi}}, \underline{\omega}, \underline{\eta})\|_{\mathcal{L}_{(\mathrm{sc})}^{4}(S)}, \\
{ }^{(S)} \mathcal{O}_{1,2}(u, \underline{u}) & =\|\nabla(\chi, \omega, \eta \widetilde{\operatorname{tr} \underline{\chi}}, \underline{\hat{\chi}}, \underline{\omega}, \underline{\eta})\|_{\mathcal{L}_{(\mathrm{sc})}^{2}(S)}, \\
{ }^{(H)} \mathcal{O}(u, \underline{u}) & =\left\|\nabla^{2}(\chi, \omega, \eta, \widetilde{\operatorname{tr} \underline{\chi}}, \underline{\hat{\chi}}, \underline{\omega}, \underline{\eta})\right\|_{\mathcal{L}_{(\mathrm{sc})}^{2}\left(H_{u}^{(0, \underline{u})}\right)} .
\end{aligned}
$$

Remark 2.13. All quantities are scale invariant except for $\widehat{\chi}$ and $\underline{\hat{\chi}}$ in the $\mathcal{L}_{(\mathrm{sc})}^{4}(S)$ norm.

As before we complement the anomalous norms for $\widehat{\chi}$ and $\underline{\hat{\chi}}$ by the local, nonanomalous, scale-invariant norms

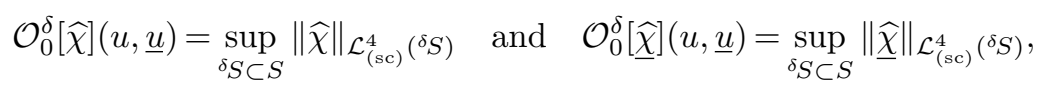

where ${ }^{\delta} S$ is a disk of radius $\delta^{1 / 2}$ obtained by transporting from the initial data embedded in $S_{u, 0}$.

$\left({ }^{3}\right)$ We use the short hand notation

$$
\|(\beta, \varrho, \sigma, \underline{\beta})\|_{\mathcal{L}_{(\mathrm{sc})}^{2}\left(H_{u}^{(0, \underline{u})}\right)}=\|\beta\|_{\mathcal{L}_{(\mathrm{sc})}^{2}\left(H_{u}^{(0, \underline{u})}\right)}+\|\varrho\|_{\mathcal{L}_{(\mathrm{sc})}^{2}\left(H_{u}^{(0, \underline{u})}\right)}+\|\sigma\|_{\mathcal{L}_{(\mathrm{sc})}^{2}\left(H_{u}^{(0, \underline{u})}\right)}+\|\underline{\beta}\|_{\mathcal{L}_{(\mathrm{sc})}^{2}\left(H_{u}^{(0, \underline{u})}\right)} \text {. }
$$




\section{Main equations. Preliminaries}

\subsection{Null structure equations}

We recall the null structure equations (see $[\mathrm{KN}, \S 3.1]$ or $[\mathrm{C}]$ ):

$$
\begin{aligned}
& \nabla_{4} \chi=-\chi \chi-2 \omega \chi-\alpha, \\
& \nabla_{3} \underline{\chi}=-\underline{\chi} \underline{\chi}-2 \underline{\omega} \underline{\chi}-\underline{\alpha}, \\
& \nabla_{4} \eta=-\chi(\eta-\underline{\eta})-\beta, \\
& \nabla_{3} \underline{\eta}=-\underline{\chi}(\underline{\eta}-\eta)+\underline{\beta}, \\
& \nabla_{4} \underline{\omega}=2 \omega \underline{\omega}+\frac{3}{4}|\eta-\underline{\eta}|^{2}-\frac{1}{4}(\eta-\underline{\eta})(\eta+\underline{\eta})-\frac{1}{8}|\eta+\underline{\eta}|^{2}+\frac{1}{2} \varrho, \\
& \nabla_{3} \omega=2 \omega \underline{\omega}+\frac{3}{4}|\eta-\underline{\eta}|^{2}+\frac{1}{4}(\eta-\underline{\eta})(\eta+\underline{\eta})-\frac{1}{8}|\eta+\underline{\eta}|^{2}+\frac{1}{2} \varrho
\end{aligned}
$$

and the constraint equations

$$
\begin{aligned}
\operatorname{div} \widehat{\chi} & =\frac{1}{2} \nabla \operatorname{tr} \chi-\frac{1}{2}(\eta-\underline{\eta})\left(\widehat{\chi}-\frac{1}{2} \operatorname{tr} \chi\right)-\beta, \\
\operatorname{div} \underline{\hat{\chi}} & =\frac{1}{2} \nabla \operatorname{tr} \underline{\chi}+\frac{1}{2}(\eta-\underline{\eta})\left(\underline{\widehat{\chi}}-\frac{1}{2} \operatorname{tr} \underline{\chi}\right)+\underline{\beta}, \\
\operatorname{curl} \eta & =-\operatorname{curl} \underline{\eta}=\sigma+\underline{\widehat{\chi}} \wedge \hat{\chi}, \\
K & =-\varrho+\frac{1}{2} \hat{\chi} \underline{\hat{\chi}}-\frac{1}{4}(\operatorname{tr} \chi) \operatorname{tr} \underline{\chi},
\end{aligned}
$$

with $K$ being the Gauss curvature of the surface $S$. The first two equations in (3.1) can also be written in the form

$$
\begin{aligned}
\nabla_{4} \operatorname{tr} \chi+\frac{1}{2}(\operatorname{tr} \chi)^{2} & =-|\widehat{\chi}|^{2}-2 \omega \operatorname{tr} \chi, \\
\nabla_{4} \hat{\chi}+(\operatorname{tr} \chi) \hat{\chi} & =-2 \omega \widehat{\chi}-\alpha, \\
\nabla_{3} \operatorname{tr} \underline{\chi}+\frac{1}{2}(\operatorname{tr} \underline{\chi})^{2} & =-2 \underline{\omega} \operatorname{tr} \underline{\chi}-|\underline{\hat{\chi}}|^{2}, \\
\nabla_{3} \underline{\hat{\chi}}+(\operatorname{tr} \underline{\chi}) \underline{\chi} & =-2 \underline{\omega} \underline{\hat{\chi}}-\underline{\alpha} .
\end{aligned}
$$

Also, with $\check{\varrho}=\varrho-\frac{1}{2} \widehat{\chi} \underline{\widehat{\chi}}$,

$$
\begin{aligned}
& \nabla_{4} \operatorname{tr} \underline{\chi}+\frac{1}{2}(\operatorname{tr} \chi) \operatorname{tr} \underline{\chi}=2 \omega \operatorname{tr} \underline{\chi}+2 \check{\varrho}+2 \operatorname{div} \underline{\eta}+2|\underline{\eta}|^{2}, \\
& \nabla_{3} \operatorname{tr} \chi+\frac{1}{2}(\operatorname{tr} \underline{\chi}) \operatorname{tr} \chi=2 \underline{\omega} \operatorname{tr} \chi+2 \check{\varrho}+2 \operatorname{div} \eta+2|\eta|^{2}
\end{aligned}
$$

$\operatorname{and}\left({ }^{4}\right)$

$$
\begin{aligned}
& \nabla_{3} \widehat{\chi}+\frac{1}{2}(\operatorname{tr} \underline{\chi}) \widehat{\chi}=\nabla \widehat{\otimes} \eta+2 \underline{\omega} \widehat{\chi}-\frac{1}{2}(\operatorname{tr} \chi) \underline{\hat{\chi}}+\eta \widehat{\otimes} \eta, \\
& \nabla_{4} \underline{\hat{\chi}}+\frac{1}{2}(\operatorname{tr} \chi) \underline{\hat{\chi}}=\nabla \underline{\widehat{\otimes}} \underline{\eta}+2 \omega \underline{\hat{\chi}}-\frac{1}{2}(\operatorname{tr} \underline{\chi}) \hat{\chi}+\underline{\eta} \widehat{\widehat{\otimes}} \underline{\eta} \text {. }
\end{aligned}
$$

$\left({ }^{4}\right)$ Recall the notation $(u \widehat{\otimes} v)_{a b}=u_{a} v_{b}+u_{b} v_{a}-(u \cdot v) \delta_{a b}$. 
Remark. The transport equations for $\omega$ and $\underline{\omega}$ in (3.1) are obtained from the null structure equation

$$
\nabla_{4} \underline{\omega}+\nabla_{3} \omega=\zeta(\eta-\underline{\eta})-\eta \underline{\eta}+4 \omega \underline{\omega}+\varrho
$$

and the commutation relation for a scalar $f$ (see [KN, Proposition 4.8.1])

$$
\left[\nabla_{3}, \nabla_{4}\right] f=-2 \omega \nabla_{3} f+2 \underline{\omega} \nabla_{4} f+4 \zeta \nabla f
$$

applied to $f=\log \Omega$.

\subsection{Null Bianchi identities}

We record below the null Bianchi identities (observe that we can eliminate $\zeta=\frac{1}{2}(\eta-\underline{\eta})$ in the equations below):

$$
\begin{aligned}
& \nabla_{3} \alpha+\frac{1}{2}(\operatorname{tr} \underline{\chi}) \alpha=\nabla \widehat{\otimes} \beta+4 \underline{\omega} \alpha-3\left(\widehat{\chi} \varrho+{ }^{*} \widehat{\chi} \sigma\right)+(\zeta+4 \eta) \widehat{\otimes} \beta, \\
& \nabla_{4} \beta+2(\operatorname{tr} \chi) \beta=\operatorname{div} \alpha-2 \omega \beta+\eta \alpha, \\
& \nabla_{3} \beta+(\operatorname{tr} \underline{\chi}) \beta=\nabla \varrho+2 \underline{\omega} \beta+{ }^{*} \nabla \sigma+2 \hat{\chi} \underline{\beta}+3\left(\eta \varrho+{ }^{*} \eta \sigma\right), \\
& \nabla_{4} \sigma+\frac{3}{2}(\operatorname{tr} \chi) \sigma=-\operatorname{div}^{*} \beta+\frac{1}{2} \underline{\chi}^{*} \alpha-\zeta^{*} \beta-2 \underline{\eta}^{*} \beta, \\
& \nabla_{3} \sigma+\frac{3}{2}(\operatorname{tr} \underline{\chi}) \sigma=-\operatorname{div}^{*} \underline{\beta}+\frac{1}{2} \widehat{\chi}^{*} \underline{\alpha}-\zeta^{*} \underline{\beta}-2 \eta^{*} \underline{\beta}, \\
& \nabla_{4} \varrho+\frac{3}{2}(\operatorname{tr} \chi) \varrho=\operatorname{div} \beta-\frac{1}{2} \underline{\hat{\chi}} \alpha+\zeta \beta+2 \underline{\eta} \beta, \\
& \nabla_{3} \varrho+\frac{3}{2}(\operatorname{tr} \underline{\chi}) \varrho=-\operatorname{div} \underline{\beta}-\frac{1}{2} \widehat{\chi} \underline{\alpha}+\zeta \underline{\beta}-2 \eta \underline{\beta}, \\
& \nabla_{4} \underline{\beta}+(\operatorname{tr} \chi) \underline{\beta}=-\nabla \varrho{ }^{*} \nabla \sigma+2 \omega \underline{\beta}+2 \underline{\widehat{\chi}} \beta-3\left(\underline{\eta} \varrho-{ }^{*} \underline{\eta} \sigma\right), \\
& \nabla_{3} \underline{\beta}+2(\operatorname{tr} \underline{\chi}) \underline{\beta}=-\operatorname{div} \underline{\alpha}-2 \underline{\omega} \underline{\beta}+\underline{\eta} \underline{\alpha}, \\
& \nabla_{4} \underline{\alpha}+\frac{1}{2}(\operatorname{tr} \chi) \underline{\alpha}=-\nabla \widehat{\otimes} \underline{\beta}+4 \omega \underline{\alpha}-3\left(\underline{\hat{\chi}} \varrho-{ }^{*} \underline{\hat{\chi}} \sigma\right)+(\zeta-4 \underline{\eta}) \widehat{\otimes} \underline{\beta} \text {. }
\end{aligned}
$$

Next, we record commutation formulas of $\nabla$ with $\nabla_{4}$ and $\nabla_{3}$.

LEMMA 3.1. For a scalar function $f$,

$$
\begin{aligned}
& {\left[\nabla_{4}, \nabla\right] f=\frac{1}{2}(\eta+\underline{\eta}) D_{4} f-\chi \nabla f,} \\
& {\left[\nabla_{3}, \nabla\right] f=\frac{1}{2}(\eta+\underline{\eta}) D_{3} f-\underline{\chi} \nabla f .}
\end{aligned}
$$

For a 1-form tangent to $S$,

$$
\begin{aligned}
& {\left[\nabla_{4}, \nabla_{a}\right] U_{b}=-\chi_{a c} \nabla_{c} U_{b}+\epsilon_{a c}{ }^{*} \beta_{b} U_{c}+\frac{1}{2}\left(\eta_{a}+\underline{\eta}_{a}\right) D_{4} U_{b}-\chi_{a c} \underline{\eta_{b}} U_{c}+\chi_{a b} \underline{\eta} U,} \\
& {\left[\nabla_{3}, \nabla_{a}\right] U_{b}=-\underline{\chi}_{a c} \nabla_{c} U_{b}+\epsilon_{a c}{ }^{*} \underline{\beta}_{b} U_{c}+\frac{1}{2}\left(\eta_{a}+\underline{\eta}_{a}\right) D_{3} U_{b}-\underline{\chi}_{a c} \eta_{b} U_{c}+\underline{\chi}_{a b} \eta U .}
\end{aligned}
$$


In particular,

$$
\begin{aligned}
& {\left[\nabla_{4}, \operatorname{div}\right] U=- } \frac{1}{2}(\operatorname{tr} \chi) \operatorname{div} U-\widehat{\chi} \nabla U+\beta U+\frac{1}{2}(\eta+\underline{\eta}) \nabla_{4} U \\
&-\underline{\eta} \hat{\chi} U-\frac{1}{2}(\operatorname{tr} \chi) \underline{\eta} U+(\operatorname{tr} \chi) \underline{\eta} U, \\
& {\left[\nabla_{3}, \operatorname{div}\right] U=- } \frac{1}{2}(\operatorname{tr} \underline{\chi}) \operatorname{div} U-\underline{\hat{\chi}} \nabla U-\underline{\beta} U+\frac{1}{2}(\eta+\underline{\eta}) \nabla_{3} U \\
&-\eta \underline{\hat{\chi}} U-\frac{1}{2}(\operatorname{tr} \underline{\chi}) \eta U+(\operatorname{tr} \underline{\chi}) \eta U .
\end{aligned}
$$

We also have, for any scalar function $f$,

$$
[L, \underline{L}] f=2(\underline{\eta}-\eta) \nabla f .
$$

\subsection{Integral formulas}

Given a scalar function $f$ in $\mathcal{D}$ we have $\left({ }^{5}\right)$

$$
\begin{aligned}
& \frac{d}{d \underline{u}} \int_{S(u, \underline{u})} f=\int_{S(u, \underline{u})}\left(\frac{d f}{d \underline{u}}+\Omega(\operatorname{tr} \chi) f\right)=\int_{S(u, \underline{u})} \Omega\left(e_{4}(f)+(\operatorname{tr} \chi) f\right), \\
& \frac{d}{d u} \int_{S(u, \underline{u})} f=\int_{S(u, \underline{u})}\left(\frac{d f}{d u}+\Omega(\operatorname{tr} \underline{\chi}) f\right)=\int_{S(u, \underline{u})} \Omega\left(e_{3}(f)+(\operatorname{tr} \underline{\chi}) f\right) .
\end{aligned}
$$

As a consequence of these, we deduce, for any horizontal tensor field $\psi$, that

$$
\begin{aligned}
& \|\psi\|_{L^{2}(S(u, \underline{u}))}^{2}=\|\psi\|_{L^{2}(S(u, 0))}^{2}+\int_{H_{u}^{(0, \underline{u})}} 2 \Omega\left(\psi \nabla_{4} \psi+\frac{1}{2}(\operatorname{tr} \chi)|\psi|^{2}\right), \\
& \|\psi\|_{L^{2}(S(u, \underline{u}))}^{2}=\|\psi\|_{L^{2}(S(0, \underline{u}))}^{2}+\int_{H_{\underline{u}}^{(u, 0)}} 2 \Omega\left(\psi \nabla_{3} \psi+\frac{1}{2}(\operatorname{tr} \underline{\chi})|\psi|^{2}\right) .
\end{aligned}
$$

Proof. The first formula in (3.10) is derived as follows:

$$
\begin{aligned}
\|\psi\|_{L^{2}(S(u, \underline{u}))}^{2} & =\|\psi\|_{L^{2}(S(u, 0))}^{2}+\int_{0}^{\underline{u}} \frac{d}{d \underline{u}}\left(\int_{S(u, \underline{u})}|\psi|^{2}\right) \\
& =\|\psi\|_{L^{2}(S(u, 0))}^{2}+\int_{H_{u}^{(0, \underline{u})}} 2 \Omega\left(\psi \nabla_{4} \psi+\frac{1}{2}(\operatorname{tr} \chi)|\psi|^{2}\right) .
\end{aligned}
$$

The second formula is proved in the same manner.

\subsection{Hodge systems}

We work with the following Hodge operators acting on the leaves $S=S(u, \underline{u})$ of our double null foliation.

$\left({ }^{5}\right)$ See for example [KN, Lemma 3.1.3]. 
(1) The operator $\mathcal{D}_{1}$ takes any 1 -form $F$ into the pairs of functions $(\operatorname{div} F, \operatorname{curl} F)$.

(2) The operator $\mathcal{D}_{2}$ takes any $S$-tangent, symmetric, traceless tensor $F$ into the $S$-tangent 1-form $\operatorname{div} F$.

(3) The operator ${ }^{\star} \mathcal{D}_{1}$ takes the pair of scalar functions $(\varrho, \sigma)$ into the $S$-tangent 1 -form $\left({ }^{6}\right)-\nabla \varrho{ }^{*} \nabla \sigma$.

(4) The operator ${ }^{\star} \mathcal{D}_{2}$ takes the 1-form $F$ on $S$ into the 2-covariant, symmetric, traceless tensor $-\frac{1}{2} \widehat{\mathcal{L}_{F} \gamma}$ with $\mathcal{L}_{F} \gamma$ being the traceless part of the Lie derivative of the metric $\gamma$ relative to $F$, i.e.

$$
{\widehat{\left(\mathcal{L}_{F} \gamma\right.}}_{a b}=\nabla_{b} F_{a}+\nabla_{a} F_{b}-(\operatorname{div} F) \gamma_{a b}
$$

The kernels of both $\mathcal{D}_{1}$ and $\mathcal{D}_{2}$ in $L^{2}(S)$ are trivial and ${ }^{\star} \mathcal{D}_{1}$, resp. ${ }^{\star} \mathcal{D}_{2}$, are the $L^{2}$ adjoints of $\mathcal{D}_{1}$, resp. $\mathcal{D}_{2}$. The kernel of ${ }^{\star} \mathcal{D}_{1}$ consists of pairs of constant functions $(\varrho, \sigma)$, while that of ${ }^{\star} \mathcal{D}_{2}$ consists of the set of all conformal Killing vector fields on $S$. In particular the $L^{2}$ range of $\mathcal{D}_{1}$ consists of all pairs of functions $(\varrho, \sigma)$ on $S$ with vanishing mean. The $L^{2}$ range of $\mathcal{D}_{2}$ consists of all $L^{2}$ integrable 1-forms on $S$ which are orthogonal to the Lie algebra of all conformal Killing vector fields on $S$. Accordingly, we shall consider the inverse operators $\mathcal{D}_{1}^{-1}$ and $\mathcal{D}_{2}^{-1}$, and implicitly assume that they are defined on the $L^{2}$ subspaces identified above.

Finally we record the following simple identities:

$$
\begin{array}{rlrl}
{ }^{\star} \mathcal{D}_{1} \mathcal{D}_{1} & =-\Delta+K, & & \mathcal{D}_{1}{ }^{\star} \mathcal{D}_{1}=-\Delta, \\
{ }^{\star} \mathcal{D}_{2} \mathcal{D}_{2} & =-\frac{1}{2} \Delta+K, & \mathcal{D}_{2}{ }^{\star} \mathcal{D}_{2}=-\frac{1}{2}(\Delta+K) .
\end{array}
$$

Proposition 3.2. Let $(S, \gamma)$ be a compact manifold with Gauss curvature $K$.

(i) The following identity holds for vector fields $\psi$ on $S$ :

$$
\int_{S}\left(|\nabla \psi|^{2}+K|\psi|^{2}\right)=\int_{S}\left(|\operatorname{div} \psi|^{2}+|\operatorname{curl} \psi|^{2}\right)=\int_{S}\left|\mathcal{D}_{1} \psi\right|^{2} .
$$

(ii) The following identity holds for symmetric, traceless, 2-tensor fields $\psi$ on $S$ :

$$
\int_{S}\left(|\nabla \psi|^{2}+2 K|\psi|^{2}\right)=2 \int_{S}|\operatorname{div} \psi|^{2}=2 \int_{S}\left|\mathcal{D}_{2} \psi\right|^{2} .
$$

(iii) The following identity holds for pairs of functions $(\varrho, \sigma)$ on $S$ :

$$
\int_{S}\left(|\nabla \varrho|^{2}+|\nabla \sigma|^{2}\right)=\int_{S}\left|-\nabla \varrho+(\nabla \sigma)^{\star}\right|^{2}=\left.\left.\int_{S}\right|^{\star} \mathcal{D}_{1}(\varrho, \sigma)\right|^{2} .
$$

(iv) The following identity holds for vectors $\psi$ on $S$ :

$$
\int_{S}\left(|\nabla \psi|^{2}-K|\psi|^{2}\right)=2 \int_{S}\left|{ }^{\star} \mathcal{D}_{2} \psi\right|^{2} .
$$

$\left({ }^{6}\right)$ Here $\left({ }^{*} \nabla \sigma\right)_{a}=\epsilon_{a b} \nabla_{b} \sigma$. 


\section{Preliminary estimates}

As explained in the introduction, the proof of Theorem A is based on the bootstrap assumption (2.13), i.e. ${ }^{(S)} \mathcal{O}_{0, \infty} \leqslant \Delta_{0}$. In this section we use this bootstrap to prove various preliminary results. In the following three sections we then derive estimates for the Ricci-coefficient norms ${ }^{(S)} \mathcal{O}_{0,4},{ }^{(S)} \mathcal{O}_{1,2}$ and ${ }^{(S)} \mathcal{O}_{1,4}$, respectively.

\subsection{Preliminary results}

We prove here results which follow easily from our bootstrap assumption ${ }^{(S)} \mathcal{O}_{0, \infty} \leqslant \Delta_{0}$. We first derive an estimate for $\Omega$. To do this, we use the definition of

$$
\underline{\omega}=-\frac{1}{2} \nabla_{3} \log \Omega=\frac{1}{2} \Omega \nabla_{3}(\Omega)^{-1}=\frac{1}{2} \frac{d}{d u}(\Omega)^{-1} .
$$

Thus, since $\Omega^{-1}=2$ on $H_{0}$,

$$
\left\|\Omega^{-1}-2\right\|_{L^{\infty}(u, \underline{u})} \lesssim \int_{0}^{u}\|\underline{\omega}\|_{L^{\infty}\left(u^{\prime}, \underline{u}\right)} d u^{\prime} \lesssim \delta^{1 / 2(S)} \mathcal{O}_{0, \infty}[\underline{\omega}] \lesssim \delta^{1 / 2} \Delta_{0} .
$$

Thus, if $\delta$ is sufficiently small, we deduce that $\left|\Omega-\frac{1}{2}\right|$ is small, and therefore

$$
\frac{1}{4} \leqslant \Omega \leqslant 4
$$

We now prove the following proposition.

Proposition 4.1. Under assumption (2.13), we have the following estimates for an arbitrary horizontal tensor field $\psi$ :

$$
\begin{aligned}
\|\psi\|_{L^{2}(u, \underline{u})} & \lesssim\|\psi\|_{L^{2}(u, 0)}+\int_{0}^{\underline{u}}\left\|\nabla_{4} \psi\right\|_{L^{2}\left(u, \underline{u}^{\prime}\right)} d \underline{u}^{\prime}, \\
\|\psi\|_{L^{2}(u, \underline{u})} & \lesssim\|\psi\|_{L^{2}(0, \underline{u})}+\int_{0}^{u}\left\|\nabla_{3} \psi\right\|_{L^{2}\left(u^{\prime}, \underline{u}\right)} d u^{\prime} .
\end{aligned}
$$

More generally, the same estimates hold in $L^{p}(S)$ norms.

Also,

$$
\begin{aligned}
\|\psi\|_{L^{2}(u, \underline{u})}^{2} & \lesssim\|\psi\|_{L^{2}(u, 0)}^{2}+\|\psi\|_{L^{2}\left(H_{u}^{(0, \underline{u})}\right)}\left\|\nabla_{4} \psi\right\|_{L^{2}\left(H_{u}^{(0, \underline{u})}\right)}, \\
\|\psi\|_{L^{2}(u, \underline{u})}^{2} & \lesssim\|\psi\|_{L^{2}(0, \underline{u})}^{2}+\|\psi\|_{L^{2}\left(\underline{H}_{\underline{u}}^{(0, u)}\right)}\left\|\nabla_{4} \psi\right\|_{L^{2}\left(\underline{H}_{\underline{H}}^{(0, u)}\right)} .
\end{aligned}
$$

Corollary 4.2. Under the same hypothesis,

$$
\begin{aligned}
\|\psi\|_{\mathcal{L}_{\text {(sc) }}^{2}(u, \underline{u})} & \lesssim\|\psi\|_{\mathcal{L}_{\text {(sc) }}^{2}(u, 0)}+\int_{0}^{\underline{u}} \delta^{-1}\left\|\nabla_{4} \psi\right\|_{\mathcal{L}_{\text {(sc) }}^{2}\left(u, \underline{u}^{\prime}\right)} d \underline{u}^{\prime}, \\
\|\psi\|_{\mathcal{L}_{\text {(sc) }}^{2}(u, \underline{u})} & \lesssim\|\psi\|_{\mathcal{L}_{\text {(sc) }}^{2}(0, \underline{u})}+\int_{0}^{u}\left\|\nabla_{3} \psi\right\|_{\mathcal{L}_{(\mathrm{sc})}^{2}\left(u^{\prime}, \underline{u}\right)} d u^{\prime}
\end{aligned}
$$


and

$$
\begin{aligned}
\|\psi\|_{\mathcal{L}_{(\mathrm{sc})}^{2}(u, \underline{u})}^{2} & \lesssim\|\psi\|_{\mathcal{L}_{(\mathrm{sc})}^{2}(u, 0)}^{2}+\|\psi\|_{\mathcal{L}_{(\mathrm{sc})}^{2}\left(H_{u}^{(0, \underline{u})}\right)}\left\|\nabla_{4} \psi\right\|_{\mathcal{L}_{(\mathrm{sc})}^{2}\left(H_{u}^{(0, \underline{u})}\right)}, \\
\|\psi\|_{\mathcal{L}_{(\mathrm{sc})}^{2}(u, \underline{u})}^{2} & \lesssim\|\psi\|_{\mathcal{L}_{(\mathrm{sc})}^{2}(0, \underline{u})}^{2}+\|\psi\|_{\mathcal{L}_{(\mathrm{sc})}^{2}\left(\underline{H}_{\underline{H}}^{(0, u)}\right)}\left\|\nabla_{4} \psi\right\|_{\mathcal{L}_{(\mathrm{sc})}^{2}\left(\underline{H}_{\underline{H}}^{(0, u)}\right)}
\end{aligned}
$$

More generally, let $S^{\prime} \subset S_{u, \underline{u}}$ and let $S_{u^{\prime}, \underline{u}}^{\prime}$ and $S_{u, \underline{u^{\prime}}}^{\prime}$ be obtained by evolving $S^{\prime}$ along the null generators of $\underline{H}_{\underline{u}}$ and $H_{u}$, respectively. Then

$$
\begin{aligned}
\|\psi\|_{\mathcal{L}_{(\mathrm{sc})}^{p}\left(S^{\prime}\right)} & \lesssim\|\psi\|_{\mathcal{L}_{(\mathrm{sc})}^{p}\left(S_{u, 0}^{\prime}\right)}+\int_{0}^{\underline{u}} \delta^{-1}\left\|\nabla_{4} \psi+\frac{1}{p}(\operatorname{tr} \chi) \psi\right\|_{\mathcal{L}_{(\mathrm{sc})}^{p}\left(S_{u, \underline{u}^{\prime}}^{\prime}\right)} d \underline{u}^{\prime}, \\
\|\psi\|_{\mathcal{L}_{(\mathrm{sc})}^{p}\left(S^{\prime}\right)} & \lesssim\|\psi\|_{\mathcal{L}_{(\mathrm{sc})}^{p}\left(S_{0, \underline{u}}^{\prime}\right)}+\int_{0}^{u}\left\|\nabla_{3} \psi+\frac{1}{p}(\operatorname{tr} \underline{\chi}) \psi\right\|_{\mathcal{L}_{(\mathrm{sc})}^{p}\left(S_{u^{\prime}, \underline{u}}^{\prime}\right)} d u^{\prime} .
\end{aligned}
$$

Proof. The corollary follows immediately from the proposition and definition of the scale-invariant norms. The last statement of the corollary follows by applying (4.4) to the function $\chi \psi$, where the cut-off function $\chi$ is first defined on $S_{u, \underline{u}}$ as the characteristic function of $S^{\prime}$ and then extended by solving the transport equations $\nabla_{4} \chi=0$ and $\nabla_{3} \chi=0$. To prove the proposition, we first make use of (4.1) and (2.13),

$$
\|\operatorname{tr} \chi\|_{L^{\infty}} \lesssim \Delta_{0} \delta^{-1 / 2}
$$

and deduce from the first equation in (3.10) that

$$
\begin{aligned}
\|\psi\|_{L^{2}(S(u, \underline{u}))}^{2} & \lesssim\|\psi\|_{L^{2}(S(u, 0))}^{2}+\int_{0}^{\underline{u}}\left(\int_{S\left(u, \underline{u}^{\prime}\right)}|\psi|\left|\nabla_{4} \psi+\frac{1}{2}(\operatorname{tr} \chi) \psi\right|\right) d \underline{u}^{\prime} \\
& \lesssim\|\psi\|_{L^{2}(S(u, 0))}^{2}+\int_{0}^{\underline{u}}\|\psi\|_{L^{2}(S)}\left(\left\|\nabla_{4} \psi\right\|_{L^{2}(S)}+\Delta_{0} \delta^{-1 / 2}\|\psi\|_{L^{2}(S)}\right) d \underline{u}^{\prime} \\
& \lesssim\|\psi\|_{L^{2}(S(u, 0))}^{2}+\int_{0}^{\underline{u}}\|\psi\|_{L^{2}(S)}\left\|\nabla_{4} \psi\right\|_{L^{2}(S)} d \underline{u}^{\prime}+\Delta_{0} \delta^{-1 / 2} \int_{0}^{\underline{u}}\|\psi\|_{L^{2}(S)}^{2} d \underline{u}^{\prime} .
\end{aligned}
$$

Thus, by Grönwall's inequality, since $\underline{u} \leqslant \delta$,

$$
\|\psi\|_{L^{2}(S(u, \underline{u}))}^{2} \lesssim\|\psi\|_{L^{2}(S(u, 0))}^{2}+\int_{0}^{\underline{u}}\left\|\nabla_{4} \psi\right\|_{L^{2}\left(u, \underline{u}^{\prime}\right)}\|\psi\|_{L^{2}\left(u, \underline{u}^{\prime}\right)} d \underline{u}^{\prime}
$$

from which we easily derive the $\nabla_{4}$ equations in both (4.2) and (4.3).

To prove the $\nabla_{3}$ estimates, we need to take into account the anomalous character of $\operatorname{tr} \underline{\chi}$. From our bootstrap assumption we deduce (recall that $\operatorname{tr} \underline{\chi}_{0}=-4 /\left(\underline{u}-u+2 r_{0}\right)$ is the flat value of $\operatorname{tr} \underline{\chi}$ ) that

$$
\left\|\operatorname{tr} \underline{\chi}-\operatorname{tr} \underline{\chi}_{0}\right\|_{L^{\infty}} \lesssim \Delta_{0} \delta^{1 / 2} .
$$


Thus,

$$
\begin{aligned}
& \|\psi\|_{L^{2}(S(u, \underline{u}))}^{2} \lesssim\|\psi\|_{L^{2}(S(0, \underline{u}))}^{2}+\int_{0}^{u}\left(\int_{S\left(u^{\prime}, \underline{u}\right)}|\psi|\left|\nabla_{3} \psi+\frac{1}{2}(\operatorname{tr} \underline{\chi}) \psi\right|\right) d u^{\prime} \\
& \lesssim\|\psi\|_{L^{2}(S(0, \underline{u}))}^{2}+\int_{0}^{u}\|\psi\|_{L^{2}(S)}\left(\left\|\nabla_{3} \psi\right\|_{L^{2}(S)}+\Delta_{0} \delta^{1 / 2}\|\psi\|_{L^{2}(S)}\right) d u^{\prime} \\
& +\int_{0}^{u}\left\|\operatorname{tr} \underline{\chi}_{0}\right\|_{L^{\infty}}\|\psi\|_{L^{2}(S)}^{2} d u^{\prime} \\
& \lesssim\|\psi\|_{L^{2}(S(0, \underline{u}))}^{2}+\int_{0}^{u}\|\psi\|_{L^{2}(S)}\left(\left\|\nabla_{3} \psi\right\|_{L^{2}(S)}+\left(1+\Delta_{0} \delta^{1 / 2}\right)\|\psi\|_{L^{2}(S)}\right) d u^{\prime} .
\end{aligned}
$$

Thus, using Grönwall's inequality and the smallness of $\delta^{1 / 2} \Delta_{0}$, we deduce that

$$
\|\psi\|_{L^{2}(S(u, \underline{u}))}^{2} \lesssim\|\psi\|_{L^{2}(S(0, \underline{u}))}^{2}+\int_{0}^{u}\|\psi\|_{L^{2}(S)}\left\|\nabla_{3} \psi\right\|_{L^{2}(S)},
$$

from which both (4.2) and (4.3) follow.

We next prove an improved estimate for $\operatorname{tr} \chi$.

Proposition 4.3. For $\delta^{1 / 2} \Delta_{0}$ sufficiently small, we have for all $S=S(u, \underline{u})$,

$$
\|\operatorname{tr} \chi\|_{L^{\infty}(S)} \lesssim \Delta_{0}^{2}
$$

Proof. We recall that $\operatorname{tr} \chi$ satisfies the transport equation

$$
\nabla_{4} \operatorname{tr} \chi=-\frac{1}{2}(\operatorname{tr} \chi)^{2}-|\widehat{\chi}|^{2}-2 \omega \operatorname{tr} \chi
$$

and

$$
\frac{d}{d \underline{u}} \operatorname{tr} \chi=-\Omega\left(\frac{1}{2}(\operatorname{tr} \chi)^{2}+|\widehat{\chi}|^{2}+2 \omega \operatorname{tr} \chi\right) .
$$

Thus, since $\|(\chi, \omega)\|_{L^{\infty}} \lesssim \delta^{-1 / 2} \Delta_{0}$,

$$
\|\operatorname{tr} \chi\|_{L^{\infty}(u, \underline{u})} \lesssim \int_{0}^{\underline{u}}\|\chi\|_{L^{\infty}\left(u, \underline{u}^{\prime}\right)}\left(\|\chi\|_{L^{\infty}\left(u, \underline{u}^{\prime}\right)}+\|\omega\|_{L^{\infty}\left(u, \underline{u}^{\prime}\right)}\right) d \underline{u}^{\prime} \lesssim \Delta_{0}^{2}+\delta^{1 / 2} \Delta_{0} .
$$

\subsection{Transported coordinates}

We define systems of local, transported coordinates along the null hypersurfaces $H$ and $\underline{H}$. Starting with a local coordinate system $\theta=\left(\theta^{1}, \theta^{2}\right)$ on $U \subset S(u, 0) \subset H_{u}$, we parameterize any point along the null geodesics starting in $U$ by the corresponding coordinate $\theta$ and affine parameter $\underline{u}$. Similarly, starting with a local coordinate system $\underline{\theta}=\left(\underline{\theta}^{1}, \underline{\theta}^{2}\right)$ on $V \subset S(0, \underline{u}) \subset \underline{H}_{\underline{u}}$, we parameterize any point along the null geodesics starting in $V$ by the corresponding coordinate $\underline{\theta}$ and affine parameter $u$. We denote the respective metric components by $\gamma_{a b}$ and $\underline{\gamma}_{a b}$. 
Proposition 4.4. Let $\gamma_{a b}^{0}$ denote the standard metric on $\mathbb{S}^{2}$. Then, for any $0 \leqslant u \leqslant 1$ and $0 \leqslant \underline{u} \leqslant \delta$ and sufficiently small $\delta^{1 / 2} \Delta_{0}$,

$$
\left|\gamma_{a b}-\gamma_{a b}^{0}\right| \leqslant \delta^{1 / 2} \Delta_{0} \quad \text { and } \quad\left|\underline{\gamma}_{a b}-\gamma_{a b}^{0}\right| \leqslant \delta^{1 / 2} \Delta_{0}
$$

In addition, the transported coordinates satisfy

$$
\left|\nabla_{3} \theta^{a}\right| \lesssim \delta \Delta_{0}, \quad\left|\nabla \theta^{a}\right| \lesssim 1, \quad\left|\nabla_{4} \underline{\theta}^{a}\right| \lesssim \delta \Delta_{0} \quad \text { and } \quad\left|\nabla \underline{\theta}^{a}\right| \lesssim 1
$$

for $a=1,2$. The Christoffel symbols $\Gamma_{a b c}$ and $\underline{\Gamma}_{a b c}$ obey the scale-invariant estimates $\left({ }^{7}\right)$

$$
\begin{aligned}
\left\|\Gamma_{a b c}\right\|_{\mathcal{L}_{(\mathrm{sc})}^{2}(S)} \lesssim \mathcal{O}_{[1]}, \quad\left\|\partial_{d} \Gamma_{a b c}\right\|_{\mathcal{L}_{(\mathrm{sc})}^{2}(S)} \lesssim \mathcal{O}_{[2]}, \\
\left\|\underline{\Gamma}_{a b c}\right\|_{\mathcal{L}_{(\mathrm{sc})}^{2}(S)} \lesssim \mathcal{O}_{[1]}, \quad\left\|\partial_{d} \underline{\Gamma}_{a b c}\right\|_{\mathcal{L}_{(\mathrm{sc})}^{2}(S)} \lesssim \mathcal{O}_{[2]}
\end{aligned}
$$

Proof. We will only show the argument in the case of $\gamma_{a b}$. In the transported coordinate system the metric $\gamma_{a b}$ satisfies

$$
\frac{d}{d \underline{u}} \gamma_{a b}=2 \Omega \chi_{a b}
$$

Therefore,

$$
\left|\gamma_{a b}-\gamma_{a b}^{0}\right| \leqslant 2 \int_{0}^{\underline{u}}\left|\chi_{a b}\right| d \underline{u}^{\prime} \leqslant \delta^{1 / 2} \Delta_{0},
$$

where in the last inequality we used that $\left|\chi_{a b}\right| \leqslant|\chi|\left|\gamma^{-1}\right|$ and ran a simple bootstrap argument.

The transported system of coordinates $\theta^{a}$ satisfies the system of equations

$$
\nabla_{4} \theta^{a}=0
$$

Commuting these equations with $\nabla_{3}$ and taking into account the commutation formula (3.6), we obtain

$$
\nabla_{4} \nabla_{3} \theta^{a}=2 \omega \nabla_{3} \theta^{a}-4 \zeta \nabla \theta^{a} .
$$

Using the bootstrap assumptions (2.13), the inequality $\left|\nabla \theta^{a}\right| \lesssim 1$ and the triviality of the data for $\nabla_{3} \theta^{a}$, we obtain that

$$
\left|\nabla_{3} \theta^{a}\right| \lesssim \delta \Delta_{0}
$$

To verify that $\left|\nabla \theta^{a}\right| \lesssim 1$, we commute the transport equation for $\theta^{a}$ with $\nabla$ to obtain, according to (3.8), that

$$
\nabla_{4} \nabla \theta^{a}=-\chi \nabla \theta^{a}
$$

$\left({ }^{7}\right)$ We can attach signatures to $\Gamma$ and $\underline{\Gamma}$, setting $\operatorname{sgn}(\Gamma)=\frac{1}{2}$ and $\operatorname{sgn}(\underline{\Gamma})=\frac{1}{2}$. 
which together with the bootstrap assumption (2.13) gives the desired result.

To prove (4.9), we differentiate the transport equation for $\gamma_{a b}$ to obtain

$$
\frac{d}{d \underline{u}}\left(\partial_{c} \gamma_{a b}\right)=2 \partial_{c} \Omega \chi_{a b}+2 \Omega \partial_{c} \chi_{a b}
$$

Taking into account that

$$
\left|\partial_{c} \Omega\right| \lesssim|\nabla \Omega| \leqslant|\eta|+|\underline{\eta}| \quad \text { and } \quad\left|\partial_{c} \chi_{a b}\right| \lesssim|\nabla \chi|+|\Gamma||\chi|,
$$

we derive that

$$
\begin{aligned}
\left\|\partial_{c} \gamma_{a b}\right\|_{L^{2}(u, \underline{u})} \lesssim & \int_{0}^{\underline{u}}\left(\|\eta\|_{L^{4}\left(u, \underline{u}^{\prime}\right)}+\|\underline{\eta}\|_{L^{4}\left(u, \underline{u}^{\prime}\right)}\right)\|\chi\|_{L^{4}\left(u, \underline{u}^{\prime}\right)} d \underline{u}^{\prime} \\
& \quad+\int_{0}^{\underline{u}}\left(\|\nabla \chi\|_{L^{2}\left(u, \underline{u}^{\prime}\right)}+\|\Gamma\|_{L^{2}\left(u, \underline{u}^{\prime}\right)}\|\chi\|_{L^{\infty}\left(u, \underline{u}^{\prime}\right)}\right) d \underline{u}^{\prime} \\
\lesssim & \delta^{3 / 4(S)} \mathcal{O}_{0,4}[\chi]^{(S)} \mathcal{O}_{0,4}[\eta, \underline{\eta}]+{ }^{(S)} \mathcal{O}_{1,2}[\chi]+\delta^{-1 / 2} \Delta_{0} \int_{0}^{\underline{u}}\|\Gamma\|_{L^{2}\left(u, \underline{u}^{\prime}\right)} d \underline{u}^{\prime} .
\end{aligned}
$$

Thus, by Grönwall's inequality,

$$
\|\Gamma\|_{L^{2}(u, \underline{u})}{ }^{(S)} \mathcal{O}_{1,2}+\delta^{3 / 4(S)} \mathcal{O}_{0,4}^{2} .
$$

The desired estimate for $\Gamma$ follows by Grönwall's inequality. The second estimate of (4.9) can be derived by an additional differentiation of the transport equation. The estimates (4.10) are proved in the same manner. We omit the details.

\subsection{Estimates for $\mathcal{R}_{0}^{\delta}[\alpha]$}

Using the transported coordinates of the previous subsection, we now derive estimates for the $\mathcal{R}_{0}^{\delta}[\alpha]$ norm of the anomalous curvature component $\alpha$.

\section{Proposition 4.5.}

$$
\mathcal{R}_{0}^{\delta}[\alpha](u) \lesssim \mathcal{R}_{0}^{\delta}[\alpha](0)+\mathcal{R} .
$$

Proof. Recall that $\mathcal{R}_{0}^{\delta}[\alpha]:=\sup _{\delta_{H \subset H}}\|\alpha\|_{\left.\mathcal{L}_{(\mathrm{sc})}^{2}{ }^{(\delta} H\right)}$, where ${ }^{\delta} H$ is the subset of $H_{u}$ generated by transporting a disk ${ }^{\delta} S$ of radius $\delta^{1 / 2}$, embedded in the sphere $S_{u, 0}$, along the integral curves of the vector field $e_{4}$. We denote by ${ }^{\delta} S_{\underline{u}}$ the intersection between ${ }^{\delta} H$ and the level hypersurfaces of $\underline{u}$, and by ${ }^{\delta} S_{u^{\prime}, \underline{u}}$ the sets obtained by transporting ${ }^{\delta} S_{\underline{u}}$ along the integral curves of $e_{3}$. According to (4.6),

$$
\|\alpha\|_{\mathcal{L}_{\text {(sc) }}^{2}\left({ }^{\delta} S_{\underline{u}}\right)} \lesssim\|\alpha\|_{\mathcal{L}_{(\mathrm{sc})}^{2}\left({ }^{\delta} S_{0, \underline{u}}\right)}+\int_{0}^{u}\left\|\nabla_{3} \alpha+\frac{1}{2}(\operatorname{tr} \underline{\chi}) \alpha\right\|_{\mathcal{L}_{(\mathrm{sc})}^{2}\left({ }^{\delta} S_{u^{\prime}, \underline{u}}\right)} d u^{\prime} .
$$


We note that (4.8) implies that ${ }^{\delta} S_{u^{\prime}, \underline{u}}$ are contained in the intersection of ${ }^{2 \delta} H_{u^{\prime}}$ and the level hypersurfaces of $\underline{u}$. Therefore,

$$
\|\alpha\|_{\mathcal{L}_{(\mathrm{sc})}^{2}\left({ }^{(} H_{u}\right)} \lesssim\|\alpha\|_{\mathcal{L}_{(\mathrm{sc})}^{2}\left({ }^{2 \delta} H_{0}\right)}+\int_{0}^{u}\left\|\nabla_{3} \alpha+\frac{1}{2}(\operatorname{tr} \underline{\chi}) \alpha\right\|_{\mathcal{L}_{(\mathrm{sc})}^{2}\left({ }^{2} H_{u^{\prime}}\right)} d u^{\prime} .
$$

Using the equation for $\alpha$,

$$
\nabla_{3} \alpha+\frac{1}{2}(\operatorname{tr} \underline{\chi}) \alpha=\nabla \widehat{\otimes} \beta+4 \underline{\omega} \alpha-3\left(\widehat{\chi} \varrho+{ }^{*} \widehat{\chi} \sigma\right)+(\zeta+4 \eta) \widehat{\otimes} \beta
$$

and the bootstrap assumptions (2.13), we obtain that

$$
\begin{aligned}
\left\|\nabla_{3} \alpha+\frac{1}{2}(\operatorname{tr} \underline{\chi}) \alpha\right\|_{\mathcal{L}_{(\mathrm{sc})}^{2}\left({ }^{2 \delta} H_{u^{\prime}}\right)} & \leqslant\left\|\nabla_{3} \alpha+\frac{1}{2}(\operatorname{tr} \underline{\chi}) \alpha\right\|_{\mathcal{L}_{(\mathrm{sc})}^{2}\left(H_{u^{\prime}}\right)} \\
& \leqslant\|\nabla \beta\|_{\mathcal{L}_{(\mathrm{sc})}^{2}\left(H_{u^{\prime}}\right)}+\delta^{1 / 2(S)} \mathcal{O}_{0, \infty} \mathcal{R}_{0} \lesssim \mathcal{R}+\delta^{1 / 2} \Delta_{0} \mathcal{R}_{0} .
\end{aligned}
$$

It remains to observe that

$$
\|\alpha\|_{\mathcal{L}_{(\mathrm{sc})}^{2}\left({ }^{2 \delta} H_{0}\right)} \lesssim \mathcal{R}_{0}^{\delta}[\alpha](u=0)
$$

which follows from a simple covering argument.

\subsection{Calculus inequalities}

Proposition 4.6. Let $(S, \gamma)$ be a compact 2-dimensional surface covered by local charts (disks) $U_{i}$, in which the metric $\gamma$ satisfies

$$
\left|\gamma_{i j}-\delta_{i j}\right| \leqslant \frac{1}{2}
$$

Let $d$ denote the minimum between 1 and the smallest radius of the disks $U_{i}$. Then, for any $p>2$,

$$
\begin{aligned}
\|\psi\|_{L^{4}(S)} & \lesssim\|\psi\|_{L^{2}(S)}^{1 / 2}\|\nabla \psi\|_{L^{2}(S)}^{1 / 2}+d^{-1 / 2}\|\psi\|_{L^{2}(S)} \\
\|\psi\|_{L^{\infty}(S)} & \lesssim\|\psi\|_{L^{p}(S)}^{p /(p+4)}\|\nabla \psi\|_{L^{p}(S)}^{4 /(p+4)}+d^{-4 /(p+4)}\|\psi\|_{L^{p}(S)}
\end{aligned}
$$

More generally,

$$
\begin{aligned}
\|\psi\|_{L^{4}\left(U_{i}\right)} & \lesssim\|\psi\|_{L^{2}\left(U_{i}\right)}^{1 / 2}\|\nabla \psi\|_{L^{2}\left(U_{i}^{\prime}\right)}^{1 / 2}+d^{-1 / 2}\|\psi\|_{L^{2}\left(U_{i}^{\prime}\right)}, \\
\|\psi\|_{L^{\infty}(S)} & \lesssim \sup _{U_{i}}\left(\|\psi\|_{L^{p}\left(U_{i}\right)}^{p /(p+4)}\|\nabla \psi\|_{L^{p}\left(U_{i}^{\prime}\right)}^{4 /(p+4)}+d^{-4 /(p+4)}\|\psi\|_{L^{p}\left(U_{i}^{\prime}\right)}\right) .
\end{aligned}
$$

Here, the disk $U_{i}^{\prime}$ is a doubled version of $U_{i}$. 
We can combine the above proposition with Proposition 4.4 to obtain the following result.

Corollary 4.7. Let $S=S_{u, \underline{u}}$ and $S_{\delta} \subset S$ denote a disk of radius $\delta^{1 / 2}$ relative to either the coordinate system $\theta$ or $\underline{\theta}$. Then, for any horizontal tensor $\psi$,

$$
\begin{aligned}
\|\psi\|_{L^{4}(S)} & \lesssim\|\psi\|_{L^{2}(S)}^{1 / 2}\|\nabla \psi\|_{L^{2}(S)}^{1 / 2}+\|\psi\|_{L^{2}(S)}, \\
\|\psi\|_{L^{\infty}(S)} & \lesssim\|\psi\|_{L^{p}(S)}^{p /(p+4)}\|\nabla \psi\|_{L^{p}(S)}^{4 /(p+4)}+\|\psi\|_{L^{p}(S)}
\end{aligned}
$$

and

$$
\begin{aligned}
\|\psi\|_{L^{4}\left(S_{\delta}\right)} & \lesssim \delta^{1 / 4}\|\nabla \psi\|_{L^{2}\left(S_{2 \delta}\right)}+\delta^{-1 / 4}\|\psi\|_{L^{2}\left(S_{2 \delta}\right)}, \\
\|\psi\|_{L^{\infty}(S)} & \lesssim \sup _{S_{\delta} \subset S}\left(\delta^{1 / 4}\|\nabla \psi\|_{L^{4}\left(S_{2 \delta}\right)}+\delta^{-1 / 4}\|\psi\|_{L^{4}\left(S_{2 \delta}\right)}\right) .
\end{aligned}
$$

Also, in the scale-invariant norms, we have the following result.

Corollary 4.8. Let $S=S_{u, \underline{u}}$ and $S_{\delta} \subset S$ denote a disk of radius $\delta^{1 / 2}$ relative to either the coordinate system $\theta$ or $\underline{\theta}$. Then, for any horizontal tensor $\psi$,

$$
\begin{aligned}
\|\psi\|_{\mathcal{L}_{(\mathrm{sc})}^{4}(S)} & \lesssim\|\psi\|_{\mathcal{L}_{(\mathrm{sc})}^{2}(S)}^{1 / 2}\|\nabla \psi\|_{\mathcal{L}_{(\mathrm{sc})}^{2}(S)}^{1 / 2}+\delta^{1 / 4}\|\psi\|_{\mathcal{L}_{(\mathrm{sc})}^{2}(S)}, \\
\|\psi\|_{\mathcal{L}_{(\mathrm{sc})}^{\infty}(S)} & \lesssim\|\psi\|_{\mathcal{L}_{(\mathrm{sc})}^{p /(p+4)}}^{p /(S)}\|\nabla \psi\|_{\mathcal{L}_{(\mathrm{sc})}^{p}(S)}^{4 /(p+4)}+\delta^{1 / p}\|\psi\|_{\mathcal{L}_{(\mathrm{sc})}^{p}(S)}
\end{aligned}
$$

and

$$
\begin{aligned}
\|\psi\|_{\mathcal{L}_{(\mathrm{sc})}^{4}\left(S_{\delta}\right)} & \lesssim\|\nabla \psi\|_{\mathcal{L}_{(\mathrm{sc})}^{2}\left(S_{2 \delta}\right)}+\|\psi\|_{\mathcal{L}_{(\mathrm{sc})}^{2}\left(S_{2 \delta}\right)}, \\
\|\psi\|_{\mathcal{L}_{(\mathrm{sc})}^{\infty}(S)} & \lesssim \sup _{S_{\delta} \subset S}\left(\|\nabla \psi\|_{\mathcal{L}_{(\mathrm{sc})}^{4}\left(S_{2 \delta}\right)}+\|\psi\|_{\mathcal{L}_{(\mathrm{sc})}^{4}\left(S_{2 \delta}\right)}\right) .
\end{aligned}
$$

\subsection{Codimension-1 trace formulas}

We will use the $L^{4}(S)$ trace formulas $\left({ }^{8}\right)$ along the null hypersurfaces $H$ and $\underline{H}$, see [CK], $[\mathrm{KN}]$ and [KR2].

LEMMA 4.9. The following formulas hold for any 2-sphere $S=S(u, \underline{u})=H(u) \cup \underline{H}(\underline{u})$ and any horizontal tensor $\psi$ :

$$
\begin{aligned}
\|\psi\|_{L^{4}(S)} & \lesssim\left(\|\psi\|_{L^{2}(H)}+\|\nabla \psi\|_{L^{2}(H)}\right)^{1 / 2}\left(\|\psi\|_{L^{2}(H)}+\left\|\nabla_{4} \psi\right\|_{L^{2}(H)}\right)^{1 / 2} \\
\|\psi\|_{L^{4}(S)} & \lesssim\left(\|\psi\|_{L^{2}(\underline{H})}+\|\nabla \psi\|_{L^{2}(\underline{H})}\right)^{1 / 2}\left(\|\psi\|_{L^{2}(\underline{H})}+\left\|\nabla_{3} \psi\right\|_{L^{2}(\underline{H})}\right)^{1 / 2}
\end{aligned}
$$

$\left.{ }^{8}\right)$ Our bootstrap assumption are more than enough to verify the conditions of validity of these estimates. 
Also, in scale-invariant norms, we have the following result.

Proposition 4.10. For a fixed $S=S(u, \underline{u})=H(u) \cap \underline{H}(\underline{u}) \subset \mathcal{D}$ and any horizontal tensor $\psi$, we have

$$
\begin{aligned}
\|\psi\|_{\mathcal{L}_{(\mathrm{sc})}^{4}(S)} & \lesssim\left(\delta^{1 / 2}\|\psi\|_{\mathcal{L}_{(\mathrm{sc})}^{2}(H)}+\|\nabla \psi\|_{\mathcal{L}_{(\mathrm{sc})}^{2}(H)}\right)^{1 / 2}\left(\delta^{1 / 2}\|\psi\|_{\mathcal{L}_{(\mathrm{sc})}^{2}(H)}+\left\|\nabla_{4} \psi\right\|_{\mathcal{L}_{\text {(sc) }}^{2}(H)}\right)^{1 / 2} \\
\|\psi\|_{\mathcal{L}_{(\mathrm{sc})}^{4}(S)} & \lesssim\left(\delta^{1 / 2}\|\psi\|_{\mathcal{L}_{(\mathrm{sc})}^{2}(\underline{H})}+\|\nabla \psi\|_{\mathcal{L}_{(\mathrm{sc})}^{2}(\underline{H})}\right)^{1 / 2}\left(\delta^{1 / 2}\|\psi\|_{\mathcal{L}_{(\mathrm{sc})}^{2}(\underline{H})}+\left\|\nabla_{3} \psi\right\|_{\mathcal{L}_{(\mathrm{sc})}^{2}(\underline{H})}\right)^{1 / 2}
\end{aligned}
$$

\subsection{Estimates for Hodge systems}

Consider a Hodge system

$$
\mathcal{D} \psi=F,
$$

with $\mathcal{D}$ being one of the operators in $\S 3.4$. In view of Proposition 3.2, we have

$$
\int_{S}|\nabla \psi|^{2}+\int_{S} K|\psi|^{2} \lesssim\|F\|_{L^{2}(S)}^{2},
$$

where

$$
K=-\varrho+\frac{1}{2} \widehat{\chi} \underline{\widehat{\chi}}-\frac{1}{4}(\operatorname{tr} \chi) \operatorname{tr} \underline{\chi}
$$

is the Gauss curvature of $S$. Hence,

$$
\|\nabla \psi\|_{L^{2}(S)}^{2} \lesssim\|K\|_{L^{2}(S)}\|\psi\|_{L^{4}(S)}^{2}+\|F\|_{L^{2}(S)}^{2} .
$$

Making use of the calculus inequality on $S$,

$$
\|\psi\|_{L^{4}(S)}^{2} \lesssim\|\nabla \psi\|_{L^{2}(S)}\|\psi\|_{L^{2}(S)},
$$

we deduce that

$$
\|\nabla \psi\|_{L^{2}(S)}^{2} \lesssim\|K\|_{L^{2}(S)}\|\nabla \psi\|_{L^{2}(S)}\|\psi\|_{L^{2}(S)}+\|F\|_{L^{2}(S)}^{2},
$$

and consequently that

$$
\|\nabla \psi\|_{L^{2}(S)} \lesssim\|K\|_{L^{2}(S)}\|\psi\|_{L^{2}(S)}+\|F\|_{L^{2}(S)} .
$$

We state below the same result in scale-invariant norms.

Proposition 4.11. Let $\psi$ satisfy the Hodge system

$$
\mathcal{D} \psi=F .
$$

Then,

$$
\|\nabla \psi\|_{\mathcal{L}_{(\mathrm{sc})}^{2}(S)} \lesssim \delta^{1 / 2}\|K\|_{\mathcal{L}_{(\mathrm{sc})}^{2}(S)}\|\psi\|_{\mathcal{L}_{(\mathrm{sc})}^{2}(S)}+\|F\|_{\mathcal{L}_{(\mathrm{sc})}^{2}(S)}
$$


To obtain the second derivative estimates for the Hodge system $\mathcal{D} \psi=F$, we apply the operator $\mathcal{D}^{*}$ and write the resulting equation schematically in the form

$$
\Delta \psi=K \psi+D^{*} F
$$

Multiplying by $\Delta \psi$, integrating over $S$ and using that $\left\|\mathcal{D}^{*} F\right\|_{L^{2}(S)} \lesssim\|\nabla F\|_{L^{2}(S)}$, we get

$$
\|\Delta \psi\|_{L^{2}(S)} \lesssim\|K\|_{L^{2}(S)}\|\psi\|_{L^{\infty}(S)}+\|\nabla F\|_{L^{2}(S)}
$$

Using Böchner's identity, see e.g. [KR2],

$$
\left\|\nabla^{2} \psi\right\|_{L^{2}(S)} \lesssim\|K\|_{L^{2}(S)}\|\psi\|_{L^{\infty}(S)}+\|K\|_{L^{2}(S)}^{1 / 2}\|\nabla \psi\|_{L^{4}(S)}+\|\Delta \psi\|_{L^{2}(S)} .
$$

We then obtain the following result.

Proposition 4.12. Let $\psi$ satisfy the Hodge system

$$
\mathcal{D} \psi=F .
$$

Then,

$\left\|\nabla^{2} \psi\right\|_{\mathcal{L}_{(\mathrm{sc})}^{2}(S)} \lesssim \delta^{1 / 2}\|K\|_{\mathcal{L}_{(\mathrm{sc})}^{2}(S)}\|\psi\|_{\mathcal{L}_{(\mathrm{sc})}^{\infty}(S)}+\delta^{1 / 4}\|K\|_{\mathcal{L}_{(\mathrm{sc})}^{2}(S)}^{1 / 2}\|\nabla \psi\|_{\mathcal{L}_{(\mathrm{sc})}^{4}(S)}+\|\nabla F\|_{\mathcal{L}_{(\mathrm{sc})}^{2}(S)}$

\section{5. ${ }^{(S)} \mathcal{O}_{0,4}$ and ${ }^{(S)} \mathcal{O}_{0,2}$ estimates}

\subsection{Estimates for $\chi, \eta$ and $\underline{\omega}$}

The null Ricci coefficients $\chi, \eta$ and $\underline{\omega}$ satisfy transport equations of the form

$$
\nabla_{4} \psi^{(s)}=\sum_{s_{1}+s_{2}=s+1} \psi^{\left(s_{1}\right)} \psi^{\left(s_{2}\right)}+\Psi^{(s+1)} .
$$

Here $\psi^{(s)}$ denotes an arbitrary Ricci-coefficient component of signature $s$, while $\Psi^{(s)}$ denotes a null curvature component of signature $s$. In view of Proposition 4.1, we have

$$
\left\|\psi^{(s)}\right\|_{\mathcal{L}_{(\mathrm{sc})}^{4}(u, \underline{u})} \lesssim\left\|\psi^{(s)}\right\|_{\mathcal{L}_{(\mathrm{sc})}^{4}(u, 0)}+\int_{0}^{\underline{u}} \delta^{-1}\left\|\nabla_{4} \psi^{(s)}\right\|_{\mathcal{L}_{(\mathrm{sc})}^{4}\left(u, \underline{u}^{\prime}\right)} d \underline{u^{\prime}} .
$$

To estimate $\left\|\nabla_{4} \psi^{(s)}\right\|_{\mathcal{L}_{(\mathrm{sc})}^{4}\left(u, \underline{u}^{\prime}\right)}$, we make use of the scale-invariant estimates

$$
\|\phi \psi\|_{\mathcal{L}_{(\mathrm{sc})}^{4}(S)} \lesssim \delta^{1 / 2}\|\phi\|_{\mathcal{L}_{(\mathrm{sc})}^{\infty}(S)}\|\psi\|_{\mathcal{L}_{(\mathrm{sc})}^{4}(S)}
$$


Hence,

$$
\left\|\nabla_{4} \psi^{(s)}\right\|_{\mathcal{L}_{(\mathrm{sc})}^{4}(S)} \lesssim\left\|\Psi^{(s+1)}\right\|_{\mathcal{L}_{(\mathrm{sc})}^{4}(S)}+\delta^{1 / 2} \sum_{s_{1}+s_{2}=s+1}\left\|\psi^{\left(s_{1}\right)}\right\|_{\mathcal{L}_{(\mathrm{sc})}^{\infty}(S)}\left\|\psi^{\left(s_{2}\right)}\right\|_{\mathcal{L}_{(\mathrm{sc})}^{4}(S)}
$$

At this point we remark that if all Ricci-coefficient and curvature norms ${ }^{(S)} \mathcal{O}_{0,4}$ and $\mathcal{R}_{0}$ were scale invariant, we would proceed in a straightforward manner as follows:

$$
\begin{aligned}
\left\|\nabla_{4} \psi^{(s)}\right\|_{\mathcal{L}_{(\mathrm{sc})}^{4}(S)} & \lesssim\left\|\Psi^{(s+1)}\right\|_{\mathcal{L}_{(\mathrm{sc})}^{4}(S)}+\delta^{1 / 2(S)} \mathcal{O}_{0, \infty}{ }^{(S)} \mathcal{O}_{0,4} \\
& \lesssim\left\|\Psi^{(s+1)}\right\|_{\mathcal{L}_{(\mathrm{sc})}^{4}(S)}+\delta^{1 / 2} \Delta_{0}{ }^{(S)} \mathcal{O}_{0,4}
\end{aligned}
$$

Hence,

$$
\begin{aligned}
\left\|\psi^{(s)}\right\|_{\mathcal{L}_{(\mathrm{sc})}^{4}(u, \underline{u})} & \lesssim\left\|\psi^{(s)}\right\|_{\mathcal{L}_{(\mathrm{sc})}^{4}(u, 0)}+\int_{0}^{\underline{u}} \delta^{-1}\left\|\Psi^{(s+1)}\right\|_{\mathcal{L}_{(\mathrm{sc})}^{4}\left(u, \underline{u}^{\prime}\right)} d \underline{u}^{\prime}+\delta^{1 / 2} \Delta_{0}{ }^{(S)} \mathcal{O}_{0,4} \\
& \lesssim\left\|\psi^{(s)}\right\|_{\mathcal{L}_{(\mathrm{sc})}^{4}(u, 0)}+\mathcal{R}_{0}^{1 / 2} \mathcal{R}_{1}^{1 / 2}+\delta^{1 / 4} \mathcal{R}_{0}+\delta^{1 / 2} \Delta_{0}{ }^{(S)} \mathcal{O}_{0,4}
\end{aligned}
$$

where in the last step we used the interpolation inequality (4.19) for the curvature $\Psi^{s+1}$. Thus, since the initial data is trivial along $\underline{u}=0$,

$$
\|(\underline{\omega}, \eta)\|_{\mathcal{L}_{(\mathrm{sc})}^{4}(u, \underline{u})} \lesssim \mathcal{R}_{0}^{1 / 2} \mathcal{R}_{1}^{1 / 2}+\delta^{1 / 4} \mathcal{R}_{0}+\delta^{1 / 2} \Delta_{0}{ }^{(S)} \mathcal{O}_{0,4}
$$

We only have to be more careful with the cases when $\left\|\Psi^{(s+1)}\right\|_{\mathcal{L}_{(\mathrm{sc})}^{4}(S)}$ is anomalous, i.e. $\Psi=\alpha$, and both $\psi^{\left(s_{1}\right)}$ and $\psi^{\left(s_{2}\right)}$ are anomalous. The first situation (but not the second) appears only in the case of the transport equation for $\hat{\chi}$, while the second one appears only in the transport equation for $\operatorname{tr} \chi$ :

$$
\nabla_{4} \widehat{\chi}+(\operatorname{tr} \chi) \widehat{\chi}=-2 \omega \widehat{\chi}-\alpha \quad \text { and } \quad \nabla_{4} \operatorname{tr} \chi+\frac{1}{2}(\operatorname{tr} \chi)^{2}=-|\widehat{\chi}|^{2}-2 \omega \operatorname{tr} \chi
$$

Thus, for a fixed $u$, we estimate, with ${ }^{\delta} S_{\underline{u}}$ denoting a disk of radius $\delta^{1 / 2}$ transported from the data at $S_{u, 0}$ (recall also the triviality of the initial data on $H_{0}$ ),

$$
\|\widehat{\chi}\|_{\mathcal{L}_{(\mathrm{sc})}^{4}\left(\delta S_{\underline{u}}\right)} \lesssim \int_{0}^{\underline{u}} \delta^{-1}\|\alpha\|_{\mathcal{L}_{(\mathrm{sc})}^{4}\left(\delta S_{u, \underline{u}^{\prime}}\right)} d \underline{u}^{\prime}+\delta^{1 / 2} \Delta_{0}{ }^{(S)} \mathcal{O}_{0,4}
$$

Using (4.21), we obtain

$$
\int_{0}^{\underline{u}} \delta^{-1}\|\alpha\|_{\mathcal{L}_{(\mathrm{sc})}^{4}\left({ }^{\delta} S_{u, \underline{u}^{\prime}}\right)} d \underline{u}^{\prime} \lesssim\|\alpha\|_{\mathcal{L}_{(\mathrm{sc})}^{2}\left({ }^{2 \delta} H_{u}^{(0, \underline{u})}\right)}+\|\nabla \alpha\|_{\mathcal{L}_{(\mathrm{sc})}^{2}\left({ }^{2 \delta} H_{u}^{(0, \underline{u})}\right)} \lesssim \mathcal{R}_{0}^{\delta}[\alpha]+\mathcal{R}_{1}[\alpha] .
$$

Therefore,

$$
\|\widehat{\chi}\|_{\mathcal{L}_{(\mathrm{sc})}^{4}\left({ }^{\delta} S_{\underline{u}}\right)} \lesssim\|\widehat{\chi}\|_{\mathcal{L}_{(\mathrm{sc})}^{4}\left({ }^{\delta} S_{0}\right)}+\mathcal{R}_{0}^{\delta}[\alpha]+\mathcal{R}_{1}[\alpha]+\delta^{1 / 2} \Delta_{0}{ }^{(S)} \mathcal{O}_{0,4}
$$


from which we derive the scale-invariant $\delta$ estimate for $\hat{\chi}$, that is

$$
{ }^{(S)} \mathcal{O}_{0,4}^{\delta}[\widehat{\chi}] \lesssim \mathcal{R}_{0}^{\delta}[\alpha]+\mathcal{R}_{1}[\alpha]+\delta^{1 / 2} \Delta_{0}{ }^{(S)} \mathcal{O}_{0,4}
$$

We can also estimate directly the anomalous ${ }^{(S)} \mathcal{O}_{0,4}[\widehat{\chi}]$ from

$$
\|\widehat{\chi}\|_{\mathcal{L}_{(\mathrm{sc})}^{4}\left(S_{\underline{u}}\right)} \lesssim \int_{0}^{\underline{u}} \delta^{-1}\|\alpha\|_{\mathcal{L}_{(\mathrm{sc})}^{4}\left({ }^{\delta} S_{u, \underline{u}^{\prime}}\right)} d \underline{u}^{\prime}+\delta^{1 / 2} \Delta_{0}{ }^{(S)} \mathcal{O}_{0,4} .
$$

Using the scale-invariant interpolation inequality (4.19), we deduce that

$$
\|\widehat{\chi}\|_{\mathcal{L}_{(\mathrm{sc})}^{4}\left(S_{\underline{u}}\right)} \lesssim\|\alpha\|_{\mathcal{L}_{(\mathrm{sc})}^{2}\left(H_{u}^{(0, \underline{u})}\right)}^{1 / 2}\|\nabla \alpha\|_{\mathcal{L}_{(\mathrm{sc})}^{2}\left(H_{u}^{(0, \underline{u})}\right)}^{1 / 2}+\delta^{1 / 4}\|\alpha\|_{\mathcal{L}_{(\mathrm{sc})}^{2}\left(H_{u}^{(0, \underline{u})}\right)}+\delta^{1 / 2} \Delta_{0}{ }^{(S)} \mathcal{O}_{0,4} .
$$

Taking into account the anomalous character of $\mathcal{R}_{0}[\alpha]$ and the definition of ${ }^{(S)} \mathcal{O}_{0,4}[\widehat{\chi}]$, we deduce that

$$
{ }^{(S)} \mathcal{O}_{0,4}[\widehat{\chi}] \lesssim \mathcal{R}_{0}[\alpha]^{1 / 2}\left(\mathcal{R}_{1}[\alpha]+\mathcal{R}_{0}[\alpha]\right)^{1 / 2}+\delta^{1 / 4} \Delta_{0}{ }^{(S)} \mathcal{O}_{0,4}
$$

On the other hand,

$$
\begin{aligned}
\|\operatorname{tr} \chi\|_{\mathcal{L}_{(\mathrm{sc})}^{4}(u, \underline{u})} \lesssim\|\operatorname{tr} \chi\|_{\mathcal{L}_{(\mathrm{sc})}^{4}(u, 0)}+\int_{0}^{\underline{u}} \delta^{-1 / 2} \Delta_{0}\|\operatorname{tr} \chi\|_{\mathcal{L}_{(\mathrm{sc})}^{4}\left(u, \underline{u}^{\prime}\right)} d \underline{u}^{\prime} \\
\quad+\delta^{-1 / 2} \Delta_{0} \int_{0}^{\underline{u}}\|\widehat{\chi}\|_{\mathcal{L}_{(\mathrm{sc})}^{4}\left(u, \underline{u}^{\prime}\right)} d \underline{u}^{\prime}+\delta^{1 / 2} \Delta_{0}{ }^{(S)} \mathcal{O}_{0,4} \\
\lesssim\|\operatorname{tr} \chi\|_{\mathcal{L}_{(\mathrm{sc})}^{4}(u, 0)}+\delta^{1 / 4} \Delta_{0}{ }^{(S)} \mathcal{O}_{0,4} .
\end{aligned}
$$

We summarize the results of this section in the following proposition. $\left({ }^{9}\right)$

Proposition 5.1. Under the bootstrap assumption ${ }^{(S)} \mathcal{O}_{0, \infty} \leqslant \Delta_{0}$ and assuming that $\delta^{1 / 2} \Delta_{0}$ is sufficiently small, we derive that

$$
\begin{aligned}
{ }^{(S)} \mathcal{O}_{0,4}[\underline{\omega}, \eta] & \lesssim \mathcal{R}_{0}+\mathcal{R}_{0}^{1 / 2} \mathcal{R}_{1}^{1 / 2}+\delta^{1 / 4} \mathcal{R}_{0}+\delta^{1 / 2} \Delta_{0}{ }^{(S)} \mathcal{O}_{0,4} \\
{ }^{(S)} \mathcal{O}_{0,4}[\operatorname{tr} \chi] & \lesssim 1+\delta^{1 / 4} \Delta_{0}{ }^{(S)} \mathcal{O}_{0,4} \\
{ }^{(S)} \mathcal{O}_{0,4}[\widehat{\chi}] & \lesssim \mathcal{R}_{0}[\alpha]^{1 / 2}\left(\mathcal{R}_{1}[\alpha]+\mathcal{R}_{0}[\alpha]\right)^{1 / 2}+\delta^{1 / 4} \Delta_{0}{ }^{(S)} \mathcal{O}_{0,4}
\end{aligned}
$$

Also,

$$
\mathcal{O}_{0}^{\delta}[\widehat{\chi}] \lesssim \mathcal{R}_{[1]}+\delta^{1 / 2} \Delta_{0}{ }^{(S)} \mathcal{O}_{0,4}
$$

$\left({ }^{9}\right)$ Recall the triviality of our initial conditions at $\underline{u}=0$. 


\subsection{Estimates for $\omega, \underline{\chi}$ and $\underline{\eta}$}

The Ricci coefficients $\underline{\chi}, \underline{\omega}$ and $\underline{\eta}$ satisfy equations of the form

$$
\nabla_{3} \psi^{(s)}=-\frac{1}{2} k(\operatorname{tr} \underline{\chi}) \psi^{(s)}+\sum_{s_{1}+s_{2}=s} \psi^{\left(s_{1}\right)} \psi^{\left(s_{2}\right)}+\Psi^{(s)},
$$

with $k$ being a positive integer. Writing $\operatorname{tr} \underline{\chi}=\operatorname{tr} \underline{\chi}_{0}+\widetilde{\operatorname{tr}} \underline{\chi}$, with $\operatorname{tr} \underline{\chi}_{0}=-4 /\left(\underline{u}-u+2 r_{0}\right)$, we derive that

$$
\nabla_{3} \psi^{(s)}=-\frac{1}{2} k\left(\operatorname{tr} \underline{\chi}_{0}\right) \psi^{(s)}+\sum_{s_{1}+s_{2}=s} \psi^{\left(s_{1}\right)} \psi^{\left(s_{2}\right)}+\Psi^{(s)}
$$

In this case, we observe that the curvature term $\Psi^{(s)}$ is never anomalous and the only time when both $\psi^{\left(s_{1}\right)}$ and $\psi^{\left(s_{2}\right)}$ are anomalous is in the case of the transport equations for $\underline{\hat{\chi}}$ and $\operatorname{tr} \underline{\chi}$. In all other cases, we can write, proceeding exactly as before,

$$
\left\|\psi^{(s)}\right\|_{\mathcal{L}_{(\mathrm{sc})}^{4}(u, \underline{u})} \lesssim\left\|\psi^{(s)}\right\|_{\mathcal{L}_{(\mathrm{sc})}^{4}(0, \underline{u})}+\int_{0}^{u}\left\|\nabla_{3} \psi^{(s)}\right\|_{\mathcal{L}_{(\mathrm{sc})}^{4}\left(u^{\prime}, \underline{u}\right)} d u^{\prime}
$$

and

$$
\left\|\nabla_{3} \psi^{(s)}\right\|_{\mathcal{L}_{(\mathrm{sc})}^{4}(u, \underline{u})} \lesssim\left\|\psi^{(s)}\right\|_{\mathcal{L}_{(\mathrm{sc})}^{4}(u, \underline{u})}+\left\|\Psi^{(s)}\right\|_{\mathcal{L}_{(\mathrm{sc})}^{4}(u, \underline{u})}+\delta^{1 / 2(S)} \mathcal{O}_{0, \infty}{ }^{(S)} \mathcal{O}_{0,4}
$$

Thus, in these cases,

$$
\begin{aligned}
\left\|\psi^{(s)}\right\|_{\mathcal{L}_{(\mathrm{sc})}^{4}(u, \underline{u})} & \lesssim\left\|\psi^{(s)}\right\|_{\mathcal{L}_{(\mathrm{sc})}^{4}(0, \underline{u})}+\int_{0}^{u}\left\|\Psi^{(s)}\right\|_{\mathcal{L}_{(\mathrm{sc})}^{4}\left(u^{\prime}, \underline{u}\right)} d u^{\prime}+\delta^{1 / 2(S)} \mathcal{O}_{0, \infty}{ }^{(S)} \mathcal{O}_{0,4} \\
& \lesssim\left\|\psi^{(s)}\right\|_{\mathcal{L}_{(\mathrm{sc})}^{4}(0, \underline{u})}+\underline{\mathcal{R}}_{0}^{1 / 2} \underline{\mathcal{R}}_{1}^{1 / 2}+\delta^{1 / 4} \underline{\mathcal{R}}_{0}+\delta^{1 / 2} \Delta_{0}{ }^{(S)} \mathcal{O}_{0,4} .
\end{aligned}
$$

Similarly,

$$
\left\|\psi^{(s)}\right\|_{\mathcal{L}_{(\mathrm{sc})}^{2}(u, \underline{u})} \lesssim\left\|\psi^{(s)}\right\|_{\mathcal{L}_{(\mathrm{sc})}^{2}(0, \underline{u})}+\underline{\mathcal{R}}_{0}+\delta^{1 / 2} \Delta_{0}{ }^{(S)} \mathcal{O}_{0,4} .
$$

It thus only remains to estimate $\operatorname{tr} \underline{\chi}$ and $\underline{\hat{\chi}}$. We first estimate $\mathcal{O}_{0}^{\delta}[\underline{\chi}]$ from the equation

$$
\nabla_{3} \underline{\hat{\chi}}=-\underline{\alpha}+\left(\operatorname{tr} \underline{\chi_{0}}\right) \underline{\hat{\chi}}-(\widetilde{\operatorname{tr}} \underline{\chi}) \underline{\hat{\chi}}-2 \underline{\omega} \underline{\hat{\chi}}
$$

Clearly, for fixed $\underline{u}$,

$$
\left\|\nabla_{3} \underline{\widehat{\chi}}+\frac{1}{2}(\operatorname{tr} \underline{\chi}) \underline{\widehat{\chi}}\right\|_{\mathcal{L}_{(\mathrm{sc})}^{4}\left({ }^{(} S_{u}\right)} \lesssim\|\underline{\alpha}\|_{\mathcal{L}_{(\mathrm{sc})}^{4}\left({ }^{(} S_{u}\right)}+\|\underline{\widehat{\chi}}\|_{\mathcal{L}_{(\mathrm{sc})}^{4}\left({ }^{\delta} S_{u}\right)}+\delta^{1 / 2(S)} \mathcal{O}_{0, \infty}{ }^{(S)} \mathcal{O}_{0,4},
$$

and thus, after a standard application of the Grönwall inequality,

$$
\|\underline{\widehat{\chi}}\|_{\mathcal{L}_{(\mathrm{sc})}^{4}\left(\delta S_{u}\right)} \lesssim\|\underline{\hat{\chi}}\|_{\mathcal{L}_{(\mathrm{sc})}^{4}\left({ }^{\delta} S_{0}\right)}+\int_{0}^{u}\|\underline{\alpha}\|_{\mathcal{L}_{(\mathrm{sc})}^{4}\left({ }^{\delta} S_{u^{\prime}}\right)} d u^{\prime} .
$$


Taking into account the scale-invariant interpolation inequality (4.19), we deduce that

$$
\|\underline{\hat{\chi}}\|_{\mathcal{L}_{(\mathrm{sc})}^{4}\left({ }^{\delta} S_{u}\right)} \lesssim\|\underline{\hat{\chi}}\|_{\mathcal{L}_{(\mathrm{sc})}^{4}\left({ }^{\delta} S_{0}\right)}+\underline{\mathcal{R}}_{0}^{1 / 2}[\underline{\alpha}] \underline{\mathcal{R}}_{1}^{1 / 2}[\underline{\alpha}]+\delta^{1 / 4} \underline{\mathcal{R}}_{0}[\underline{\alpha}]+\delta^{1 / 2} \Delta_{0}{ }^{(S)} \mathcal{O}_{0,4}
$$

and, since $\|\underline{\hat{\chi}}\|_{\mathcal{L}_{(\mathrm{sc})}^{4}\left({ }^{\delta} S_{0}\right)} \lesssim \mathcal{O}^{(0)}$,

$$
\|\underline{\hat{\chi}}\|_{\mathcal{L}_{(\mathrm{sc})}^{4}\left({ }^{\delta} S_{u}\right)} \lesssim \mathcal{O}^{(0)}+\underline{\mathcal{R}}_{0}^{1 / 2}[\underline{\alpha}]\left(\underline{\mathcal{R}}_{1}^{1 / 2}[\underline{\alpha}]+\delta^{1 / 4} \underline{\mathcal{R}}_{0}[\underline{\alpha}]\right)+\delta^{1 / 2} \Delta_{0}{ }^{(S)} \mathcal{O}_{0,4} .
$$

Proceeding in the same fashion,

$$
\|\underline{\hat{\chi}}\|_{\mathcal{L}_{(\mathrm{sc})}^{4}\left(S_{u}\right)} \lesssim\|\underline{\hat{\chi}}\|_{\mathcal{L}_{(\mathrm{sc})}^{4}\left(S_{0}\right)}+\underline{\mathcal{R}}_{0}^{1 / 2}[\underline{\alpha}] \underline{\mathcal{R}}_{1}^{1 / 2}[\underline{\alpha}]+\delta^{1 / 4} \underline{\mathcal{R}}_{0}[\underline{\alpha}]+\delta^{1 / 2} \Delta_{0}{ }^{(S)} \mathcal{O}_{0,4}
$$

Now, observe that the only anomaly on the right-hand side is due to $\|\underline{\widehat{x}}\|_{\mathcal{L}_{(\mathrm{sc})}^{4}\left(S_{0}\right)}$. In fact,

$$
\|\underline{\widehat{\chi}}\|_{\mathcal{L}_{(\mathrm{sc})}^{4}\left(S_{0}\right)} \lesssim \delta^{-1 / 4} \mathcal{O}^{(0)}
$$

Thus,

$$
{ }^{(S)} \mathcal{O}_{0,4}[\underline{\widehat{\chi}}] \lesssim \mathcal{O}^{(0)}+\delta^{1 / 4} \underline{\mathcal{R}}_{0}^{1 / 2}[\underline{\alpha}] \underline{\mathcal{R}}_{1}^{1 / 2}[\underline{\alpha}]+\delta^{1 / 2} \underline{\mathcal{R}}_{0}+\delta^{3 / 4} \Delta_{0}{ }^{(S)} \mathcal{O}_{0,4}
$$

To estimate $\widetilde{\operatorname{tr}} \underline{\chi}=\operatorname{tr} \underline{\chi}-\operatorname{tr} \underline{\chi}_{0}$, we start with the equation

$$
D_{3} \operatorname{tr} \underline{\chi}+\frac{1}{2}(\operatorname{tr} \underline{\chi})^{2}=-2 \underline{\omega} \operatorname{tr} \underline{\chi}-|\underline{\chi}|^{2}
$$

Since $D_{3} u=\Omega^{-1}, D_{3} \underline{u}=0$ and $\operatorname{tr} \underline{\chi}_{0}=-4 /\left(\underline{u}-u+2 r_{0}\right)$, we have

$$
D_{3} \operatorname{tr} \underline{\chi}_{0}=-\frac{1}{4} \Omega^{-1}\left(\operatorname{tr} \underline{\chi}_{0}\right)^{2}
$$

Hence, using $\widetilde{\operatorname{tr}} \underline{\chi}=\operatorname{tr} \underline{\chi}-\operatorname{tr} \underline{\chi}_{0}$,

$$
\nabla_{3} \widetilde{\operatorname{tr} \underline{\chi}}+\left(\operatorname{tr} \underline{\chi}_{0}\right) \widetilde{\operatorname{tr} \underline{\chi}}=-\frac{1}{2 \Omega}\left(\Omega-\frac{1}{2}\right)\left(\operatorname{tr} \underline{\chi}_{0}\right)^{2}+2 \underline{\omega} \operatorname{tr} \underline{\chi}_{0}-2 \underline{\omega} \widetilde{\operatorname{tr} \underline{\chi}}-|\underline{\widehat{\chi}}|^{2}
$$

Now, taking into account the anomalous scaling of ${ }^{(S)} \mathcal{O}_{0,4}[\underline{\hat{\chi}}]$ and the estimate

$$
\left\|\Omega-\frac{1}{2}\right\|_{\mathcal{L}_{(\mathrm{sc})}^{2}(S)} \lesssim\|\underline{\omega}\|_{\mathcal{L}_{(\mathrm{sc})}^{2}(S)}
$$

(which can be easily derived using the transport equation $\nabla_{3} \Omega=\underline{\omega}$ ), we derive that

$$
\left\|\nabla_{3} \widetilde{\operatorname{tr} \underline{\chi}}\right\|_{\mathcal{L}_{(\mathrm{sc})}^{4}(S)} \lesssim\|\widetilde{\operatorname{tr} \underline{\chi}}\|_{\mathcal{L}_{(\mathrm{sc})}^{4}(S)}+\|\underline{\omega}\|_{\mathcal{L}_{(\mathrm{sc})}^{4}(S)}+\delta^{1 / 4(S)} \mathcal{O}_{0, \infty}{ }^{(S)} \mathcal{O}_{0,4}
$$


from which

$$
\begin{aligned}
& \|\widetilde{\operatorname{tr} \underline{\chi}}\|_{\mathcal{L}_{(\mathrm{sc})}^{4}(u, \underline{u})} \lesssim\|\widetilde{\operatorname{tr} \underline{\underline{\chi}}}\|_{\mathcal{L}_{(\mathrm{sc})}^{4}(0, \underline{u})}+\int_{0}^{u}\left\|\nabla_{3} \widetilde{\operatorname{tr} \underline{\chi}}\right\|_{\mathcal{L}_{(\mathrm{sc})}^{4}\left(u^{\prime}, \underline{u}\right)} d u^{\prime} \\
& \lesssim\|\widetilde{\operatorname{tr} \underline{\chi}}\|_{\mathcal{L}_{(\mathrm{sc})}^{4}(0, \underline{u})}+\int_{0}^{u}\|\widetilde{\operatorname{tr} \underline{\chi}}\|_{\mathcal{L}_{(\mathrm{sc})}^{4}\left(u^{\prime}, \underline{u}\right)} d u^{\prime} \\
& +\int_{0}^{u}\|\underline{\omega}\|_{\mathcal{L}_{(\mathrm{sc})}^{4}\left(u^{\prime}, \underline{u}\right)} d u^{\prime}+\delta^{1 / 4} \Delta_{0}{ }^{(S)} \mathcal{O}_{0,4} .
\end{aligned}
$$

By Grönwall's inequality, and using the estimate for $\underline{\omega}$ derived in the previous section, we have

$$
\|\widetilde{\operatorname{tr} \underline{\chi}}\|_{\mathcal{L}_{\text {(sc) }}^{4}(u, \underline{u})} \lesssim\|\widetilde{\operatorname{tr} \underline{\chi}}\|_{\mathcal{L}_{\text {(sc) }}^{4}(0, \underline{u})}+\mathcal{R}_{0}^{1 / 2} \mathcal{R}_{1}^{1 / 2}+\delta^{1 / 4} \mathcal{R}_{0}+\delta^{1 / 4} \Delta_{0}{ }^{(S)} \mathcal{O}_{0,4}
$$

Thus,

$$
{ }^{(S)} \mathcal{O}_{0,4}[\widetilde{\operatorname{tr} \underline{\chi}}] \lesssim \mathcal{O}^{(0)}+\mathcal{R}_{0} \mathcal{R}_{1}+\delta^{1 / 4} \mathcal{R}_{0}+\delta^{1 / 4} \Delta_{0}{ }^{(S)} \mathcal{O}_{0,4} .
$$

We summarize the result of this subsection in the following result.

Proposition 5.2. We have, for sufficiently small $\delta$,

$$
\begin{aligned}
{ }^{(S)} \mathcal{O}_{0,4}[\underline{\eta}, \omega] & \lesssim \mathcal{O}^{(0)}+\underline{\mathcal{R}}_{0}+\underline{\mathcal{R}}_{0}^{1 / 2} \underline{\mathcal{R}}_{1}^{1 / 2}+\delta^{1 / 4} \underline{\mathcal{R}}_{0}+\delta^{1 / 2} \Delta_{0}{ }^{(S)} \mathcal{O}_{0,4}, \\
{ }^{(S)} \mathcal{O}_{0,4}[\underline{\hat{\chi}}] & \lesssim \mathcal{O}^{(0)}+\delta^{1 / 4} \underline{\mathcal{R}}_{0}^{1 / 2} \underline{\mathcal{R}}_{1}^{1 / 2}+\delta^{1 / 2} \underline{\mathcal{R}}_{0}+\delta^{3 / 4} \Delta_{0}{ }^{(S)} \mathcal{O}_{0,4} \\
{ }^{(S)} \mathcal{O}_{0,4}[\widetilde{\operatorname{tr}} \underline{\underline{\chi}}] & \lesssim \mathcal{O}^{(0)}+\mathcal{R}_{0} \mathcal{R}_{1}+\delta^{1 / 4} \mathcal{R}_{0}+\delta^{1 / 4} \Delta_{0}{ }^{(S)} \mathcal{O}_{0,4}
\end{aligned}
$$

Also,

$$
\mathcal{O}^{\delta}[\underline{\hat{\chi}}] \lesssim \mathcal{O}^{(0)}+\underline{\mathcal{R}}_{0}^{1 / 2} \underline{\mathcal{R}}_{1}^{1 / 2}+\delta^{1 / 4} \underline{\mathcal{R}}_{0}+\delta^{1 / 2} \Delta_{0}{ }^{(S)} \mathcal{O}_{0,4}
$$

\subsection{Summary of ${ }^{(S)} \mathcal{O}_{0,4}$ estimates}

Putting together the last two propositions, we deduce the following result.

Proposition 5.3. There exists a constant $C$ depending only on $\mathcal{O}^{(0)}$ and $\mathcal{R}$ such that, if $\delta^{1 / 2} \Delta_{0}$ is sufficiently small, we have

$$
{ }^{(S)} \mathcal{O}_{0,4} \lesssim C
$$

Moreover,

$$
\begin{aligned}
& { }^{(S)} \mathcal{O}_{0,4}[\hat{\chi}] \lesssim \mathcal{R}_{0}[\alpha]^{1 / 2}\left(\mathcal{R}_{1}[\alpha]+\mathcal{R}_{0}[\alpha]\right)^{1 / 2}+\delta^{1 / 4} C, \\
& { }^{(S)} \mathcal{O}_{0,4}[\underline{\widehat{\chi}}] \lesssim \mathcal{O}^{(0)}+\delta^{1 / 4} C
\end{aligned}
$$




\section{4. ${ }^{(S)} \mathcal{O}_{0,2}$ estimates}

The following estimates will also be needed.

Proposition 5.4. There exists a constant $C$ depending only on $\mathcal{O}^{(0)}$ and $\mathcal{R}$ such that, if $\delta^{1 / 2} \Delta_{0}$ is sufficiently small, we have

$$
{ }^{(S)} \mathcal{O}_{0,2} \lesssim C
$$

Proof. This is similar but somewhat simpler, once we already have the ${ }^{(S)} \mathcal{O}_{0,4}$ estimates. Indeed, starting with (5.1) (dropping indices for simplicity), we write as before

$$
\|\psi\|_{\mathcal{L}_{(\mathrm{sc})}^{2}(u, \underline{u})} \lesssim\|\psi\|_{\mathcal{L}_{(\mathrm{sc})}^{2}(u, 0)}+\int_{0}^{\underline{u}} \delta^{-1}\left\|\nabla_{4} \psi\right\|_{\mathcal{L}_{(\mathrm{sc})}^{2}\left(u, \underline{u}^{\prime}\right)} d \underline{u}^{\prime}
$$

and, assuming the worst case scenario when both factors in $\psi \psi$ are anomalous, i.e. both satisfy $\|\psi\|_{\mathcal{L}_{(\mathrm{sc})}^{4}(S)} \lesssim C \delta^{-1 / 4}$

$$
\begin{aligned}
\left\|\nabla_{4} \psi\right\|_{\mathcal{L}_{(\mathrm{sc})}^{2}(S)} & \lesssim\|\Psi\|_{\mathcal{L}_{(\mathrm{sc})}^{2}(S)}+\delta^{1 / 2}\|\psi\|_{\mathcal{L}_{(\mathrm{sc})}^{4}(S)}\|\psi\|_{\mathcal{L}_{(\mathrm{sc})}^{4}(S)} \\
& \lesssim\|\Psi\|_{\mathcal{L}_{(\mathrm{sc})}^{2}(S)}+{ }^{(S)} \mathcal{O}_{0,4}^{2} \lesssim\|\Psi\|_{\mathcal{L}_{(\mathrm{sc})}^{2}(S)}+C^{2}
\end{aligned}
$$

Thus,

$$
\|\psi\|_{\mathcal{L}_{(\mathrm{sc})}^{2}(u, \underline{u})} \lesssim+\int_{0}^{\underline{u}} \delta^{-1}\|\Psi\|_{\mathcal{L}_{(\mathrm{sc})}^{2}\left(u, \underline{u}^{\prime}\right)} d \underline{u}^{\prime}+C^{2} \lesssim\|\Psi\|_{\mathcal{L}_{(\mathrm{sc})}^{2}\left(H_{u}\right)}+C^{2} .
$$

$\Psi$ can only be the anomalous $\alpha$ in the case of the transport equation for $\widehat{\chi}$. Thus,

$$
\|(\underline{\omega}, \eta)\|_{\mathcal{L}_{(\mathrm{sc})}^{2}(u, \underline{u})} \lesssim \mathcal{R}_{0}+C^{2} \quad \text { and } \quad\|\widehat{\chi}\|_{\mathcal{L}_{(\mathrm{sc})}^{2}(u, \underline{u})} \lesssim \delta^{-1 / 2} \mathcal{R}_{0}[\alpha]
$$

or, with a constant $C=C\left(\mathcal{O}^{(0)}, \mathcal{R}, \underline{\mathcal{R}}\right)$,

$$
{ }^{(S)} \mathcal{O}_{0,2}[\operatorname{tr} \chi, \widehat{\chi}, \underline{\omega}, \eta] \lesssim C .
$$

The estimates for $\operatorname{tr} \underline{\chi}, \underline{\widehat{\chi}}, \omega$ and $\underline{\eta}$ are proved in the same manner.

\section{6. $\mathcal{O}_{1}$ estimates}

\subsection{General strategy}

To get the first and second derivative estimates for the Ricci coefficients we cannot proceed as we did in the previous section. Following a path first pursued in $[\mathrm{CK}]$ and continued in $[\mathrm{KN}],[\mathrm{KR} 1]$ and $[\mathrm{C}]$, we introduce new quantities $\left({ }^{10}\right) \Theta^{(s)}$, with signature $s$,

$\left({ }^{10}\right)$ Different components $\Theta$ appear in (6.1) and (6.2). It may in fact be more appropriate to denote by $\Theta$ the components which appear on the left of the $\nabla_{4}$ equation and by $\underline{\Theta}$ those appearing on the left of the $\nabla_{3}$ equations. 
depending on the first derivative of the Ricci coefficients and which satisfy transport equations of the form $\left({ }^{11}\right)$

$$
\begin{aligned}
& \nabla_{4} \Theta^{(s)}=(\operatorname{tr} \chi)\left(\Theta^{(s)}+\nabla \psi^{(s-1 / 2)}\right)+\sum_{s_{1}+s_{2}+1 / 2=s+1} \psi^{\left(s_{1}\right)}\left(\nabla \psi^{\left(s_{2}\right)}+\Psi^{\left(s_{2}\right)}\right) \\
&+\sum_{s_{1}+s_{2}=s+1}\left(\operatorname{tr} \underline{\chi}_{0}\right) \psi^{\left(s_{1}\right)} \psi^{\left(s_{2}\right)}+\sum_{s_{1}+s_{2}+s_{3}=s+1} \psi^{\left(s_{1}\right)} \psi^{\left(s_{2}\right)} \psi^{\left(s_{3}\right)}, \\
& \nabla_{3} \Theta^{(s)}=(\operatorname{tr} \underline{\chi})\left(\Theta^{(s)}+\nabla \psi^{(s-1 / 2)}\right)+\sum_{s_{1}+s_{2}+1 / 2=s} \psi^{\left(s_{1}\right)}\left(\nabla \psi^{\left(s_{2}\right)}+\Psi^{\left(s_{2}\right)}\right) \\
& \\
&+\sum_{s_{1}+s_{2}=s}\left(\operatorname{tr} \underline{\chi}_{0}\right) \psi^{\left(s_{1}\right)} \psi^{\left(s_{2}\right)}+\sum_{s_{1}+s_{2}+s_{3}=s} \psi^{\left(s_{1}\right)} \psi^{\left(s_{2}\right)} \psi^{\left(s_{3}\right)} .
\end{aligned}
$$

Here $\psi^{(s)}$ are components of all the Ricci coefficients $\operatorname{tr} \chi, \widehat{\chi}, \omega, \eta, \widetilde{\operatorname{tr}} \underline{\chi}, \underline{\hat{\chi}}, \underline{\omega}$ and $\underline{\eta}$ with signature $s$, while $\Psi^{(s)}$ are curvature components with signature $s$.

The main idea behind our strategy is to show that once we control the $\mathcal{L}_{(\mathrm{sc})}^{2}(S)$ norms of these quantities $\Theta$, we derive all $\mathcal{O}_{1}$ estimates by using the elliptic Hodge systems. The most general form of such systems is given by

$$
\mathcal{D} \psi^{(s)}=\Theta^{(s+1 / 2)}+\Psi^{(s+1 / 2)}+\left(\operatorname{tr} \underline{\chi}_{0}\right) \psi^{(s+1 / 2)}+\sum_{s_{1}+s_{2}=s+1 / 2} \psi^{\left(s_{1}\right)} \psi^{\left(s_{2}\right)},
$$

where $\mathcal{D}$ is one of the Hodge systems of $\S 3.4$. Observe also that both Hodge systems have non-anomalous curvature source terms $\beta$ and $\underline{\beta}$, respectively, and no quadratic anomalies in $\psi$ (relative to the $\mathcal{O}_{0}$ norm).

\subsection{Explicit $\Theta$ variables and Hodge systems}

In this section we introduce explicit variables $\Theta^{(s)}$ and derive transport equations of type (6.1) and (6.2).

Transport-Hodge systems for $\chi$ and $\underline{\chi}$. First observe that the Codazzi equations

$$
\begin{aligned}
& \operatorname{div} \hat{\chi}=\frac{1}{2} \nabla \operatorname{tr} \chi-\frac{1}{2}(\eta-\underline{\eta})\left(\widehat{\chi}-\frac{1}{2} \operatorname{tr} \chi\right)-\beta, \\
& \operatorname{div} \underline{\hat{\chi}}=\frac{1}{2} \nabla \operatorname{tr} \underline{\chi}+\frac{1}{2}(\eta-\underline{\eta})\left(\underline{\hat{\chi}}-\frac{1}{2} \operatorname{tr} \underline{\chi}\right)+\underline{\beta}
\end{aligned}
$$

can be written as Hodge systems of type (6.3), with $\mathcal{D}$ being the Hodge operator $\mathcal{D}_{2}$, discussed in $\S 3.4$, and $\Theta=\nabla \operatorname{tr} \chi$, resp. $\Theta=\nabla \operatorname{tr} \underline{\chi}$.

(11) We neglect to write possible constants in front of each term on the right of our equations. 
We now derive a $\nabla_{4}$ transport equation for $\nabla \operatorname{tr} \chi$. Using the commutation formula $\left[\nabla_{4}, \nabla\right] f=\frac{1}{2}(\eta+\underline{\eta}) D_{4} f-\chi \nabla f$, we obtain that

$$
\begin{aligned}
\nabla_{4} \nabla \operatorname{tr} \chi=- & (\nabla \operatorname{tr} \chi) \operatorname{tr} \chi-2(\operatorname{tr} \chi) \nabla \omega-2 \omega \nabla \operatorname{tr} \chi-2(\nabla \hat{\chi}) \hat{\chi} \\
& +\frac{1}{2}(\eta+\underline{\eta})\left(-\frac{1}{2}(\operatorname{tr} \chi)^{2}-2 \omega \operatorname{tr} \chi-|\widehat{\chi}|^{2}\right)-\chi \nabla \operatorname{tr} \chi,
\end{aligned}
$$

which is clearly of the form (6.1) with no curvature terms present and no triple anomalies (relative to the $\mathcal{O}_{0}$ norm, i.e. among the cubic terms at least one of the factors is not anomalous).

To derive a transport equation for $\nabla \operatorname{tr} \underline{\chi}$, we start with the transport equation

$$
\nabla_{3} \operatorname{tr} \underline{\chi}=-\frac{1}{2}(\operatorname{tr} \underline{\chi})^{2}+F, \quad F=-2 \underline{\omega} \operatorname{tr} \underline{\chi}-|\underline{\hat{\chi}}|^{2}=-2 \underline{\omega} \operatorname{tr} \underline{\chi} \underline{\chi}_{0}-2 \underline{\omega} \widetilde{\operatorname{tr}} \underline{\chi}-|\underline{\hat{\chi}}|^{2} .
$$

Using the commutator formula $\left[\nabla_{3}, \nabla\right] f=-\underline{\chi} \nabla f+\frac{1}{2}(\eta+\underline{\eta}) D_{3} f$, we deduce that

$$
\nabla_{3} \nabla \operatorname{tr} \underline{\chi}=-\underline{\hat{\chi}} \nabla \operatorname{tr} \underline{\chi}-\frac{3}{2}(\operatorname{tr} \underline{\chi}) \nabla \operatorname{tr} \underline{\chi}-\left(\nabla+\frac{1}{2}(\eta+\underline{\eta})\right) F-\frac{1}{4}(\eta+\underline{\eta})(\operatorname{tr} \underline{\chi})^{2},
$$

that is, writing $\operatorname{tr} \underline{\chi}=\operatorname{tr} \underline{\chi}_{0}+\widetilde{\operatorname{tr} \underline{\chi}}$,

$$
\begin{aligned}
\nabla_{3} \nabla \operatorname{tr} \underline{\chi}=-\underline{\widehat{\chi}} \nabla \operatorname{tr} \underline{\chi}-\frac{3}{2}\left(\operatorname{tr} \underline{\chi}_{0}\right) \nabla \operatorname{tr} \underline{\chi}-\frac{3}{2}(\widetilde{\operatorname{tr} \underline{\chi}}) \nabla \operatorname{tr} \underline{\chi} \\
-\left(\nabla+\frac{1}{2}(\eta+\underline{\eta})\right) F-\frac{1}{4}(\eta+\underline{\eta})(\operatorname{tr} \underline{\chi})^{2} .
\end{aligned}
$$

This is clearly a system of the form (6.2) with no curvature terms present and no anomalous cubic terms.

Transport-Hodge systems for $\mu, \underline{\mu}, \nabla \eta$ and $\nabla \underline{\eta}$. We start with the equation

$$
\operatorname{curl} \eta=-\operatorname{curl} \underline{\eta}=\sigma+\underline{\hat{\chi}} \wedge \hat{\chi} .
$$

We get equations for $\operatorname{div} \eta$ and $\operatorname{div} \underline{\eta}$ by taking the divergence of the transport equations:

$$
\begin{aligned}
& \nabla_{4} \eta=-\frac{1}{2}(\operatorname{tr} \chi)(\eta-\underline{\eta})-\widehat{\chi}(\eta-\underline{\eta})-\beta, \\
& \nabla_{3} \underline{\eta}=-\frac{1}{2}(\operatorname{tr} \underline{\chi})(\underline{\eta}-\eta)-\underline{\widehat{\chi}}(\underline{\eta}-\eta)+\underline{\beta} .
\end{aligned}
$$

Using the commutation Lemma 3.1, we derive that

$$
\begin{aligned}
\nabla_{4} \operatorname{div} \eta= & \operatorname{div}\left(-\frac{1}{2}(\operatorname{tr} \chi)(\eta-\underline{\eta})-\widehat{\chi}(\eta-\underline{\eta})-\beta\right)-\frac{1}{2}(\operatorname{tr} \chi) \operatorname{div} \eta-\widehat{\chi} \nabla \eta-\eta \beta+\frac{1}{2}(\eta+\underline{\eta}) \nabla_{4} \eta \\
=- & \operatorname{div} \beta-\frac{1}{2}(\operatorname{tr} \chi)(2 \operatorname{div} \eta-\operatorname{div} \underline{\eta})-(\eta-\underline{\eta})\left(\frac{1}{2} \nabla \operatorname{tr} \chi+\operatorname{div} \hat{\chi}\right) \\
& -\widehat{\chi} \nabla(2 \eta-\underline{\eta})-\eta \beta+\frac{1}{2}(\eta+\underline{\eta}) \nabla_{4} \eta .
\end{aligned}
$$


Using the null Codazzi equation

$$
\frac{1}{2} \nabla \operatorname{tr} \chi+\operatorname{div} \widehat{\chi}=\nabla \operatorname{tr} \chi+\frac{1}{2} \zeta \operatorname{tr} \chi-\beta,
$$

we derive that

$$
\begin{aligned}
\nabla_{4} \operatorname{div} \eta=- & \operatorname{div} \beta-\frac{1}{2}(\operatorname{tr} \chi)(2 \operatorname{div} \eta-\operatorname{div} \underline{\eta})-\widehat{\chi} \nabla(2 \eta-\underline{\eta})-(\eta-\underline{\eta}) \nabla \operatorname{tr} \chi \\
& -\underline{\eta} \beta-\frac{1}{4}(\operatorname{tr} \chi)(\eta-\underline{\eta})^{2}+\frac{1}{2}(\eta+\underline{\eta})\left(-\frac{1}{2}(\operatorname{tr} \chi)(\eta-\underline{\eta})-\widehat{\chi}(\eta-\underline{\eta})-\beta\right) \\
& +\frac{1}{2}(\eta-\underline{\eta}) \widehat{\chi}(\eta-\underline{\eta}) \\
=- & \operatorname{div} \beta-\frac{1}{2}(\operatorname{tr} \chi)(2 \operatorname{div} \eta-\operatorname{div} \underline{\eta})-\widehat{\chi} \nabla(2 \eta-\underline{\eta})-(\eta-\underline{\eta}) \nabla \operatorname{tr} \chi \\
& -\frac{1}{2}(3 \underline{\eta}+\eta) \beta-\frac{1}{2}(\operatorname{tr} \chi)\left(|\eta|^{2}-\eta \underline{\eta}\right)-\frac{1}{2}(\eta+\underline{\eta}) \widehat{\chi}(\eta-\underline{\eta})+\frac{1}{2}(\eta-\underline{\eta}) \widehat{\chi}(\eta-\underline{\eta}),
\end{aligned}
$$

that is

$$
\begin{aligned}
\nabla_{4} \operatorname{div} \eta+(\operatorname{tr} \chi) \operatorname{div} \eta=- & \operatorname{div} \beta+\frac{1}{2}(\operatorname{tr} \chi) \operatorname{div} \underline{\eta}-\widehat{\chi} \nabla(2 \eta-\underline{\eta})-(\eta-\underline{\eta}) \nabla \operatorname{tr} \chi \\
& -\frac{1}{2}(3 \underline{\eta}+\eta) \beta-\frac{1}{2}(\operatorname{tr} \chi)\left(|\eta|^{2}-\eta \underline{\eta}\right)-\frac{1}{2}(\eta+\underline{\eta}) \widehat{\chi}(\eta-\underline{\eta}) \\
& +\frac{1}{2}(\eta-\underline{\eta}) \widehat{\chi}(\eta-\underline{\eta}) .
\end{aligned}
$$

On the other hand,

$$
\nabla_{4} \varrho+\frac{3}{2}(\operatorname{tr} \chi) \varrho=\operatorname{div} \beta-\frac{1}{2} \underline{\widehat{\chi}} \alpha+\zeta \beta+2 \underline{\eta} \beta .
$$

Adding the two equations and setting $\mu=-\operatorname{div} \eta-\varrho$, we get

$$
\begin{aligned}
\nabla_{4} \mu+(\operatorname{tr} \chi) \mu=- & \frac{1}{2}(\operatorname{tr} \chi) \operatorname{div} \underline{\eta}+(\eta-\underline{\eta}) \nabla \operatorname{tr} \chi+\widehat{\chi} \nabla(2 \eta-\underline{\eta})+\frac{1}{2} \underline{\hat{\chi}} \alpha-(\eta-3 \underline{\eta}) \beta+\frac{1}{2}(\operatorname{tr} \chi) \varrho \\
& +\frac{1}{2}(\operatorname{tr} \chi)\left(|\eta|^{2}-\eta \underline{\eta}\right)+\frac{1}{2}(\eta+\underline{\eta}) \widehat{\chi}(\eta-\underline{\eta}) .
\end{aligned}
$$

Similarly, setting $\underline{\mu}=-\operatorname{div} \underline{\eta}-\varrho$, we derive that

$$
\begin{aligned}
\nabla_{3} \underline{\mu}+(\operatorname{tr} \underline{\chi}) \underline{\mu}=- & \frac{1}{2}(\operatorname{tr} \underline{\chi}) \operatorname{div} \eta+(\underline{\eta}-\eta) \nabla \operatorname{tr} \underline{\chi}+\underline{\hat{\chi}} \nabla(2 \underline{\eta}-\eta)+\frac{1}{2} \hat{\chi} \underline{\alpha}-(\underline{\eta}-3 \eta) \underline{\beta}+\frac{1}{2}(\operatorname{tr} \underline{\chi}) \varrho \\
& +\frac{1}{2}(\operatorname{tr} \underline{\chi})\left(|\underline{\eta}|^{2}-\underline{\eta} \eta\right)+\frac{1}{2}(\eta+\underline{\eta}) \underline{\widehat{\chi}}(\underline{\eta}-\eta) .
\end{aligned}
$$

We summarize the results above in the following lemma.

LEMma 6.1. The reduced mass aspect functions

$$
\mu=-\operatorname{div} \eta-\varrho \quad \text { and } \quad \underline{\mu}=-\operatorname{div} \underline{\eta}-\varrho
$$

satisfy the transport equations

$$
\begin{aligned}
\nabla_{4} \mu+(\operatorname{tr} \chi) \mu=- & \frac{1}{2}(\operatorname{tr} \chi) \operatorname{div} \underline{\eta}+(\eta-\underline{\eta}) \nabla \operatorname{tr} \chi+\hat{\chi} \nabla(2 \eta-\underline{\eta})+\frac{1}{2} \underline{\widehat{\chi}} \alpha-(\eta-3 \underline{\eta}) \beta+\frac{1}{2}(\operatorname{tr} \chi) \varrho \\
& +\frac{1}{2}(\operatorname{tr} \chi)\left(|\eta|^{2}-\eta \underline{\eta}\right)+\frac{1}{2}(\eta+\underline{\eta}) \hat{\chi}(\eta-\underline{\eta}) \\
\nabla_{3} \underline{\mu}+(\operatorname{tr} \underline{\chi}) \underline{\mu}=- & \frac{1}{2}(\operatorname{tr} \underline{\chi}) \operatorname{div} \eta+(\underline{\eta}-\eta) \nabla \operatorname{tr} \underline{\chi}+\underline{\hat{\chi}} \nabla(2 \underline{\eta}-\eta)+\frac{1}{2} \widehat{\chi} \underline{\alpha}-(\underline{\eta}-3 \eta) \underline{\beta}+\frac{1}{2}(\operatorname{tr} \underline{\chi}) \varrho \\
& +\frac{1}{2}(\operatorname{tr} \underline{\chi})\left(|\underline{\eta}|^{2}-\underline{\eta} \eta\right)+\frac{1}{2}(\eta+\underline{\eta}) \underline{\widehat{\chi}}(\underline{\eta}-\eta) .
\end{aligned}
$$


Remark 6.2. Observe that our mass aspect functions differ from those of [CK] and $[\mathrm{KN}]$. Thus, in $[\mathrm{KN}], \mu=-\operatorname{div} \eta-\varrho+\frac{1}{2} \widehat{\chi} \underline{\chi}$ satisfies (see [KN, equation (4.3.32)])

$$
\begin{gathered}
\nabla_{4} \mu+(\operatorname{tr} \chi) \mu=\widehat{\chi}(\nabla \widehat{\otimes} \eta)+(\eta-\underline{\eta})(\nabla \operatorname{tr} \chi+(\operatorname{tr} \chi) \zeta)+\frac{1}{2}(\operatorname{tr} \chi)(\mu+\operatorname{div}(\eta-\underline{\eta})) \\
-\frac{1}{4}(\operatorname{tr} \underline{\chi})|\widehat{\chi}|^{2}+\frac{1}{2}(\operatorname{tr} \chi)\left(\widehat{\chi} \underline{\hat{\chi}}+2 \varrho-|\underline{\eta}|^{2}\right)+2(\eta \widehat{\chi} \underline{\eta}-\eta \beta) .
\end{gathered}
$$

The reason we prefer our definition here is to avoid the presence of triple anomalous terms on the right-hand side of the transport equations for $\mu$ and $\mu$.

We write (6.8) symbolically in the form

$$
\nabla_{4} \mu=\psi\left(\nabla \psi+\Psi_{g}\right)+\underline{\widehat{\chi}} \alpha+\psi \psi \psi_{g}
$$

which is of the form (6.1), with $\psi_{g} \in\{\operatorname{tr} \chi, \widehat{\chi}, \omega, \eta, \operatorname{tr} \underline{\chi}, \underline{\omega}, \underline{\eta}\}$ and $\Psi_{g} \in\{\beta, \varrho, \sigma, \underline{\beta}\}$. We can also write, in shorter form,

$$
\nabla_{4} \mu=\psi(\nabla \psi+\Psi)+\psi \psi \psi_{g}
$$

and recall that $\psi \Psi$ contains the more difficult term $\underline{\widehat{\chi}} \alpha$ anomalous in both $\psi$ and $\Psi$.

We also rewrite (6.9) symbolically. In this case we have to keep track of the terms proportional to $\operatorname{tr} \underline{\chi}=\operatorname{tr} \underline{\chi}_{0}+\widetilde{\operatorname{tr}} \underline{\chi}$. We thus write, symbolically,

$$
\nabla_{3} \underline{\mu}=\left(\operatorname{tr} \underline{\chi}_{0}\right)(\nabla \psi+\underline{\mu})+\psi\left(\nabla \psi+\Psi_{g}\right)+\psi_{g} \underline{\beta}+\left(\operatorname{tr} \underline{\chi}_{0}\right) \psi \psi_{g}+\psi \psi \psi_{g}
$$

Here $\Psi_{g} \in\{\varrho, \sigma, \underline{\alpha}, \underline{\beta}\}$. Observe that at least one of the factors $\psi$ in $\left(\operatorname{tr} \underline{\chi}_{0}\right) \psi \psi_{g}$ and $\psi \psi \psi_{g}$ can be anomalous. Unlike in the case of the $\nabla_{4} \mu$ equation, there are no terms of the form $\psi \beta$ with $\psi$ also anomalous (recall that $\beta$ is anomalous for $\underline{\mathcal{R}}_{0}$ ).

We combine the transport equations (6.10) and (6.11) with the Hodge systems,

$$
\begin{gathered}
\operatorname{div} \eta=-\mu-\varrho, \\
\operatorname{curl} \eta=\sigma-\frac{1}{2} \widehat{\chi} \wedge \underline{\widehat{\chi}}
\end{gathered}
$$

and

$$
\begin{aligned}
\operatorname{div} \underline{\eta} & =-\underline{\mu}-\varrho, \\
\operatorname{curl} \underline{\eta} & =-\sigma+\frac{1}{2} \widehat{\chi} \wedge \underline{\widehat{\chi}} .
\end{aligned}
$$

They are both systems of type (6.3). Note that the quadratic term $\widehat{\chi} \underline{\hat{\chi}}$ is anomalous with respect to both factors.

Transport-Hodge systems for $\varkappa, \underline{\varkappa}, \nabla \omega$ and $\nabla \underline{\omega}$. We look for transport equations for quantities connected to $\nabla \omega$ and $\nabla \underline{\omega}$. Recall that

$$
\nabla_{4} \underline{\omega}=\frac{1}{2} \varrho+F, \quad F=2 \omega \underline{\omega}+\frac{3}{4}|\eta-\underline{\eta}|^{2}-\frac{1}{4}(\eta-\underline{\eta})(\eta+\underline{\eta})-\frac{1}{8}|\eta+\underline{\eta}|^{2}
$$


and

$$
\nabla_{3} \omega=\frac{1}{2} \varrho+\underline{F}, \quad \underline{F}=2 \omega \underline{\omega}+\frac{3}{4}|\eta-\underline{\eta}|^{2}+\frac{1}{4}(\eta-\underline{\eta})(\eta+\underline{\eta})-\frac{1}{8}|\eta+\underline{\eta}|^{2} .
$$

We introduce the auxiliary quantities $\underline{\omega}^{\dagger}$ and $\omega^{\dagger}$ by

$$
\begin{aligned}
& \nabla_{4} \underline{\omega}^{\dagger}=\frac{1}{2} \sigma \\
& \nabla_{3} \omega^{\dagger}=\frac{1}{2} \sigma
\end{aligned}
$$

with zero boundary conditions along $\underline{H}_{0}$ and $H_{0}$, respectively. We introduce the pair of scalars $\langle\underline{\omega}\rangle=\left(\underline{\omega}, \underline{\omega}^{\dagger}\right)$ and $\langle\omega\rangle=\left(-\omega, \omega^{\dagger}\right)$, and apply the Hodge operator ${ }^{\star} \mathcal{D}_{1}$ (see $\left.\S 3.4\right)$

$$
{ }^{\star} \mathcal{D}_{1}\langle\underline{\omega}\rangle=-\nabla \underline{\omega}+{ }^{*} \nabla \underline{\omega}^{\dagger} \text { and } \quad{ }^{\star} \mathcal{D}_{1}\langle\omega\rangle=\nabla \omega+{ }^{*} \nabla \omega^{\dagger} .
$$

Next we derive a $\nabla_{4}$ equation for $\langle\underline{\omega}\rangle$ and a $\nabla_{3}$ equation for $\langle\omega\rangle$. To do this, we write the commutation relation (3.9) in the form

$$
\begin{gathered}
{\left[\nabla_{4}, \nabla\right] f=-\frac{1}{2}(\operatorname{tr} \chi) \nabla f-\widehat{\chi} \nabla f+\frac{1}{2}(\eta+\underline{\eta}) D_{4} f} \\
{\left[\nabla_{4},{ }^{*} \nabla\right] g=-\frac{1}{2}(\operatorname{tr} \chi)^{*} \nabla g+\widehat{\chi}^{*} \nabla g+\frac{1}{2}\left(\eta^{*}+\underline{\eta}^{*}\right) D_{4} g .}
\end{gathered}
$$

Thus, for a pair of scalars $(f, g)$,

$$
\left[\nabla_{4},{ }^{\star} \mathcal{D}_{1}\right](f, g)=-\frac{1}{2}(\operatorname{tr} \chi)^{\star} \mathcal{D}_{1}(f, g)+\widehat{\chi}\left(\nabla f+{ }^{*} \nabla g\right)-\frac{1}{2}(\eta+\underline{\eta}) \nabla_{4} f+\frac{1}{2}\left(\eta^{*}+\underline{\eta}^{*}\right) D_{4} g .
$$

Therefore,

$$
\begin{aligned}
\nabla_{4}{ }^{\star} \mathcal{D}_{1}\langle\underline{\omega}\rangle={ }^{\star} \mathcal{D}_{1}(\varrho, \sigma)-\nabla F+\left[\nabla_{4},{ }^{\star} \mathcal{D}_{1}\right]\langle\underline{\omega}\rangle \\
={ }^{\star} \mathcal{D}_{1}(\varrho, \sigma)-\nabla F-\frac{1}{2}(\operatorname{tr} \chi){ }^{\star} \mathcal{D}_{1}\langle\underline{\omega}\rangle+\widehat{\chi}\left(\nabla \underline{\omega}+{ }^{*} \nabla \underline{\omega}^{\dagger}\right) \\
\quad-\frac{1}{2}(\eta+\underline{\eta})(\varrho+F)+\frac{1}{2}\left(\eta^{*}+\underline{\eta}^{*}\right) \sigma .
\end{aligned}
$$

On the other hand, we have the Bianchi equation

$$
D_{4} \underline{\beta}+(\operatorname{tr} \chi) \underline{\beta}={ }^{\star} \mathcal{D}_{1}(\varrho, \sigma)+2 \omega \underline{\beta}+2 \underline{\hat{\chi}} \beta-3\left(\underline{\eta} \varrho-{ }^{*} \underline{\eta} \sigma\right)
$$

Thus, introducing the new horizontal vector

$$
\underline{\varkappa}:={ }^{\star} \mathcal{D}_{1}\langle\underline{\omega}\rangle-\frac{1}{2} \underline{\beta}={ }^{\star} \mathcal{D}_{1}\left(\underline{\omega}, \underline{\omega}^{\dagger}\right)-\frac{1}{2} \underline{\beta}=-\nabla \underline{\omega}+{ }^{*} \nabla \underline{\omega}^{\dagger}-\frac{1}{2} \underline{\beta},
$$

we deduce that

$$
\begin{aligned}
\nabla_{4} \underline{\varkappa}=- & (\operatorname{tr} \chi) \underline{\varkappa}-\omega \underline{\beta}-\underline{\widehat{\chi}} \beta+\frac{3}{2}\left(\underline{\eta} \varrho-{ }^{*} \eta \sigma\right)-\frac{1}{2}(\eta+\underline{\eta}) \varrho+\frac{1}{2}\left(\eta^{*}+\underline{\eta}^{*}\right) \sigma \\
& +\widehat{\chi}\left(\nabla \underline{\omega}+{ }^{*} \nabla \underline{\omega}^{\dagger}\right)-\nabla F-\frac{1}{2}(\eta+\underline{\eta}) F .
\end{aligned}
$$


Similarly we set

$$
\varkappa:={ }^{\star} \mathcal{D}_{1}\langle\omega\rangle-\frac{1}{2} \beta={ }^{\star} \mathcal{D}_{1}\left(-\omega, \omega^{\dagger}\right)-\frac{1}{2} \beta=\nabla \omega+{ }^{*} \nabla \omega \dagger-\frac{1}{2} \beta
$$

and, using the Bianchi equations

$$
D_{3} \beta+(\operatorname{tr} \underline{\chi}) \beta=\mathcal{D}_{1}^{*}(-\varrho, \sigma)+2 \underline{\omega} \beta+2 \widehat{\chi} \underline{\beta}+3\left(\eta \varrho+{ }^{*} \eta \sigma\right),
$$

we derive that

$$
\begin{aligned}
\nabla_{3} \varkappa=- & (\operatorname{tr} \underline{\chi}) \varkappa-\underline{\omega} \beta-\widehat{\chi} \underline{\beta}+\frac{3}{2}\left(\eta \varrho{ }^{*} \eta \sigma\right)-\frac{1}{2}(\underline{\eta}+\eta) \varrho+\frac{1}{2}\left(\underline{\eta}^{*}+\eta^{*}\right) \sigma \\
& +\underline{\widehat{\chi}}\left(-\nabla \omega+{ }^{*} \nabla \omega^{\dagger}\right)+\nabla \underline{F}+\frac{1}{2}(\underline{\eta}+\eta) \underline{F} .
\end{aligned}
$$

To estimate $\nabla \underline{\omega}$, we combine the $\nabla_{4}$ equation (6.18) with the Hodge system

$$
{ }^{\star} \mathcal{D}_{1}\left(\underline{\omega}, \underline{\omega}^{\dagger}\right)=\underline{\varkappa}+\frac{1}{2} \underline{\beta} .
$$

And t6o estimate $\nabla \omega$, we combine the $\nabla_{3}$ equation (6.19) with the Hodge system

$$
{ }^{\star} \mathcal{D}_{1}\left(-\omega, \omega^{\dagger}\right)=\varkappa+\frac{1}{2} \beta \text {. }
$$

Clearly, the transport equations for $\underline{\varkappa}$ and $\varkappa$ are of the form (6.1) and (6.2) provided that we extend the set of Ricci coefficients $\psi$ to also include the new scalars $\underline{\omega}^{\dagger}$ and $\omega^{\dagger}$. We observe that $\underline{\omega}^{\dagger}$ has the same signature as $\underline{\omega}$, and $\omega^{\dagger}$ has the same signature as $\omega$. Moreover $\underline{\omega}^{\dagger}$ and $\omega^{\dagger}$ satisfy equations similar to those satisfied by $\underline{\omega}$ and $\omega$. Thus, for example, we can easily derive both $\mathcal{L}_{(\mathrm{sc})}^{2}$ and $\mathcal{L}_{(\mathrm{sc})}^{4}$ estimates for them. Indeed, from (6.15), we easily derive that

$$
\left\|\underline{\omega}^{\dagger}\right\|_{\mathcal{L}_{(\mathrm{sc})}^{2}(u, \underline{u})} \lesssim \int_{0}^{\underline{u}} \delta^{-1}\|\sigma\|_{\mathcal{L}_{(\mathrm{sc})}^{2}\left(u, \underline{u}^{\prime}\right)} d \underline{u}^{\prime} \lesssim \mathcal{R}_{0}[\sigma] .
$$

Similarly, from (6.16),

$$
\left\|\omega^{\dagger}\right\|_{\mathcal{L}_{(\mathrm{sc})}^{2}(u, \underline{u})} \lesssim \int_{0}^{u}\|\sigma\|_{\mathcal{L}_{(\mathrm{sc})}^{2}\left(u^{\prime}, \underline{u}\right)} d u^{\prime} \lesssim \underline{\mathcal{R}}_{0}[\sigma] .
$$

It thus makes perfect sense to extend the definition of the set of Ricci coefficients as well as the definition of the norms ${ }^{(S)} \mathcal{O}_{\infty},{ }^{(S)} \mathcal{O}_{0,4},{ }^{(S)} \mathcal{O}_{1,2}$ and ${ }^{(S)} \mathcal{O}_{1,4}$ to include them. We therefore also assume, from now on, that the main bootstrap assumption (2.13) includes $\underline{\omega}^{\dagger}$ and $\omega^{\dagger}$.

Finally we observe that equations (6.18) and (6.19) can be written in the form

$$
\begin{aligned}
& \nabla_{4} \underline{\varkappa}=-(\operatorname{tr} \chi) \underline{\varkappa}+\psi\left(\Psi_{g}+\nabla \psi\right)+\psi \psi \psi_{g}, \\
& \nabla_{3} \varkappa=-(\operatorname{tr} \underline{\chi}) \varkappa+\psi\left(\Psi_{g}+\nabla \psi\right)+\psi \psi \psi_{g},
\end{aligned}
$$

with $\Psi_{g} \in\{\beta, \varrho, \sigma, \underline{\beta}\}$ and $\psi_{g} \in\left\{\operatorname{tr} \chi, \omega, \omega^{\dagger}, \eta, \widetilde{\operatorname{tr}} \underline{\chi}, \underline{\omega}, \underline{\omega}^{\dagger}, \underline{\eta}\right\}$. Since $\underline{\varkappa}$ can be expressed in terms of $\nabla \omega, \nabla \omega^{\dagger}$ and $\underline{\beta}$, we can also write the first equation in the form

$$
\nabla_{4} \underline{\varkappa}=\psi\left(\Psi_{g}+\nabla \psi\right)+\psi \psi \psi_{g} .
$$

The second equation can be written in the form

$$
\nabla_{3} \varkappa=-\left(\operatorname{tr} \underline{\chi}_{0}\right) \varkappa+\psi\left(\Psi_{g}+\nabla \psi\right)+\psi \psi \psi_{g} .
$$




\subsection{Main $\mathcal{O}_{1}$ estimates}

We start by rewriting systems (6.1), (6.2) and (6.3) in short form, dropping the reference to the signature,

$$
\begin{aligned}
& \nabla_{4} \Theta=\psi(\nabla \psi+\Psi)+\left(\operatorname{tr} \underline{\chi}_{0}\right) \psi \psi_{g}+\psi \psi \psi_{g} \\
& \nabla_{3} \Theta=\left(\operatorname{tr} \underline{\chi}_{0}\right) \nabla \psi+\psi(\nabla \psi+\Psi)+\left(\operatorname{tr} \underline{\chi}_{0}\right) \psi \psi_{g}+\psi \psi \psi_{g}
\end{aligned}
$$

where $\psi_{g}$ denotes an extended Ricci-coefficient term (i.e. including $\underline{\omega}^{\dagger}$ and $\omega^{\dagger}$ defined below) which is not anomalous in the ${ }^{(S)} \mathcal{O}_{0,4}$-norm. Also,

$$
\mathcal{D} \psi=\Theta+\Psi+\left(\operatorname{tr} \underline{\chi}_{0}\right) \psi_{g}+\psi \psi \text {. }
$$

Remark 6.3. In reality equation (6.22) should also contain a term of the form $\operatorname{tr} \underline{\chi}_{0} \Theta$, as seen in (6.7), (6.11) and (6.20). We observe however that such terms can be easily eliminated by a standard Grönwall inequality.

Remark 6.4. The curvature terms $\Psi$ appearing on the right-hand side of (6.21) belong to the admissible $\left({ }^{12}\right)$ set $\{\alpha, \beta, \varrho, \sigma, \underline{\beta}\}$. Special attention needs to be given to terms of the form $\left({ }^{13}\right) \underline{\widehat{\chi}} \alpha$.

Remark 6.5. The curvature terms $\Psi$ appearing on the right-hand side of $(6.22)$ belong to the admissible $\left({ }^{14}\right)$ set $\{\beta, \varrho, \sigma, \underline{\alpha}, \underline{\beta}\}$. Special attention needs to be given to terms of the form $\psi \beta$, since $\underline{\mathcal{R}}_{0}[\beta]$ is anomalous. We observe however that among all possible terms of the form $\psi \beta, \psi$ is never anomalous.

Remark 6.6. The curvature terms $\Psi$ appearing on the right-hand side of $(6.23)$ belong to the set $\{\beta, \varrho, \sigma, \underline{\beta}\}$.

Remark 6.7. $\psi_{g}$ denotes an extended Ricci coefficient which is not anomalous in the $\mathcal{O}_{0}$ norm. Whenever we write simply $\psi$, we allow for the possibility that it may be anomalous. For example the terms of the form $\psi \psi$ in (6.23) may be both anomalous (as happens to be the case for the div-curl systems for $\eta$ and $\underline{\eta}$, due to $\hat{\chi} \underline{\hat{\chi}}$ ).

Remark 6.8. Due to the triviality of our initial data at $\underline{u}=0$, we have

$$
\|\Theta\|_{\mathcal{L}_{(\mathrm{sc})}^{2}(u, 0)}=0 .
$$

In view of the definition of $\Theta$, we have

$$
\|\Theta\|_{\mathcal{L}_{(\mathrm{sc})}^{2}(0, \underline{u})} \lesssim \mathcal{O}^{(0)}+\mathcal{R}^{(0)}
$$

\footnotetext{
(12) This are the curvature components appearing in the main curvature norms $\mathcal{R}_{0}$ and $\mathcal{R}_{1}$.

(13) Such a term appear in the transport equation for $\mu$

$\left.{ }^{14}\right)$ This are the curvature components appearing in the main curvature norms $\underline{\mathcal{R}}_{0}$ and $\underline{\mathcal{R}}_{1}$.
} 
We start deriving estimates for (6.21). As in the proof of the $\mathcal{O}_{0}$ estimates

$$
\|\Theta\|_{\mathcal{L}_{(\mathrm{sc})}^{2}(u, \underline{u})} \lesssim\|\Theta\|_{\mathcal{L}_{(\mathrm{sc})}^{2}(u, 0)}+\int_{0}^{\underline{u}} \delta^{-1}\left\|\nabla_{4} \Theta\right\|_{\mathcal{L}_{(\mathrm{sc})}^{2}\left(u, \underline{u}^{\prime}\right)} d \underline{u}^{\prime} .
$$

Recall that none of the $\mathcal{L}_{(\mathrm{sc})}^{\infty}(S)$ norms of the Ricci coefficients $\psi$ or the $\mathcal{L}_{(\mathrm{sc})}^{2}(S)$ norms of their derivatives $\nabla \psi$ are anomalous. Moreover,

$$
\left\|\psi_{g}\right\|_{\mathcal{L}_{(\mathrm{sc})}^{4}(S)}+\delta^{1 / 4}\left\|\psi_{g}\right\|_{\mathcal{L}_{(\mathrm{sc})}^{4}(S)} \lesssim{ }^{(S)} \mathcal{O}_{0,4}(S) \lesssim C
$$

where $C$ is the constant in Proposition 5.3. Also,

$$
\|\psi\|_{\mathcal{L}_{(\mathrm{sc})}^{\infty}(S)} \lesssim \delta^{1 / 2} \Delta_{0} \quad \text { and } \quad\|\nabla \psi\|_{\mathcal{L}_{(\mathrm{sc})}^{2}(S)} \lesssim{ }^{(S)} \mathcal{O}_{1,2}
$$

Now, according to $(6.21)$, for $\delta^{1 / 2} \Delta_{0} \lesssim 1$,

$$
\begin{aligned}
&\left\|\nabla_{4} \Theta\right\|_{\mathcal{L}_{(\mathrm{sc})}^{2}(S) \lesssim}\|\psi \Psi\|_{\mathcal{L}_{(\mathrm{sc})}^{2}(S)}+\delta^{1 / 2}\|\psi\|_{\mathcal{L}_{(\mathrm{sc})}^{\infty}(S)}\|\nabla \psi\|_{\mathcal{L}_{(\mathrm{sc})}^{2}(S)} \\
& \quad+\delta^{1 / 2}\|\psi\|_{\mathcal{L}_{(\mathrm{sc})}^{4}(S)}\left\|\psi_{g}\right\|_{\mathcal{L}_{(\mathrm{sc})}^{4}(S)}+\delta\|\psi\|_{\mathcal{L}_{(\mathrm{sc})}^{\infty}(S)}\|\psi\|_{\mathcal{L}_{(\mathrm{sc})}^{4}(S)}\left\|\psi_{g}\right\|_{\mathcal{L}_{(\mathrm{sc})}^{4}(S)} \\
& \lesssim\|\psi \Psi\|_{\mathcal{L}_{(\mathrm{sc})}^{2}(S)}+\delta^{1 / 2} \Delta_{0}\|\nabla \psi\|_{\mathcal{L}_{(\mathrm{sc})}^{2}(S)}+\delta^{1 / 4} C^{2}
\end{aligned}
$$

Recalling the triviality of the initial conditions at $\underline{u}=0$, we deduce that

$$
\begin{aligned}
\|\Theta\|_{\mathcal{L}_{(\mathrm{sc})}^{2}(u, \underline{u})} & \lesssim \int_{0}^{\underline{u}} \delta^{-1}\left\|\nabla_{4} \Theta\right\|_{\mathcal{L}_{(\mathrm{sc})}^{2}\left(u, \underline{u}^{\prime}\right)} d \underline{u}^{\prime} \\
& \lesssim \delta^{-1} \int_{0}^{\underline{u}}\|\psi \Psi\|_{\mathcal{L}_{(\mathrm{sc})}^{2}\left(u, \underline{u}^{\prime}\right)} d \underline{u}^{\prime}+\Delta_{0} \delta^{1 / 2(S)} \mathcal{O}_{1,2}+\delta^{1 / 4} C^{2} .
\end{aligned}
$$

Among the terms of the form $\psi \Psi$, the most dangerous $\left({ }^{15}\right)$ is $\underline{\hat{\chi}} \alpha$ which is anomalous in both $\psi$ and $\Psi$. In this case, recalling estimate (5.7),

$$
\|\underline{\underline{\chi}}\|_{\mathcal{L}_{(\mathrm{sc})}^{4}(S)} \lesssim \delta^{-1 / 4} C
$$

we deduce that

$$
\begin{aligned}
\|\underline{\hat{\chi}} \alpha\|_{\mathcal{L}_{(\mathrm{sc})}^{2}(S)} & \lesssim \delta^{1 / 2}\|\underline{\hat{\chi}}\|_{\mathcal{L}_{(\mathrm{sc})}^{4}(S)}\|\alpha\|_{\mathcal{L}_{(\mathrm{sc})}^{4}(S)} \\
& \lesssim \delta^{1 / 4} C\left(\|\nabla \alpha\|_{\mathcal{L}_{(\mathrm{sc})}^{2}(S)}^{1 / 2}\|\alpha\|_{\mathcal{L}_{(\mathrm{sc})}^{2}(S)}^{1 / 2}+\delta^{1 / 4}\|\alpha\|_{\mathcal{L}_{(\mathrm{sc})}^{2}(S)}\right) .
\end{aligned}
$$

All other terms are better in powers of $\delta$, i.e.

$$
\|\psi \Psi\|_{\mathcal{L}_{(\mathrm{sc})}^{2}(S)} \lesssim \delta^{1 / 4} C\left(\|\Psi\|_{\mathcal{L}_{(\mathrm{sc})}^{2}(S)}^{1 / 2}\|\nabla \Psi\|_{\mathcal{L}_{(\mathrm{sc})}^{2}(S)}^{1 / 2}+\delta^{1 / 4}\|\Psi\|_{\mathcal{L}_{(\mathrm{sc})}^{2}(S)}\right) .
$$

$\left({ }^{15}\right)$ This is the case for the $\nabla_{4}$ equation for $\mu$. 
Therefore, recalling Remark 6.4 and the definition of the scale-invariant norms $\mathcal{L}_{(\mathrm{sc})}^{2}\left(H_{u}\right)$,

$$
\begin{aligned}
\delta^{-1} \int_{0}^{\underline{u}}\|\psi \Psi\|_{\mathcal{L}_{(\mathrm{sc})}^{2}\left(u, \underline{u}^{\prime}\right)} d \underline{u}^{\prime} & \lesssim C \delta^{-3 / 4} \int_{0}^{\underline{u}}\|\Psi\|_{\mathcal{L}_{(\mathrm{sc})}^{2}\left(u, \underline{u}^{\prime}\right)}^{1 / 2}\|\nabla \Psi\|_{\mathcal{L}_{(\mathrm{sc})}^{2}\left(u, \underline{u}^{\prime}\right)}^{1 / 2} d \underline{u}^{\prime} \\
& \lesssim C \delta^{-1 / 4}\left(\int_{0}^{\underline{u}}\|\Psi\|_{\mathcal{L}_{(\mathrm{sc})}^{2}\left(u, \underline{u}^{\prime}\right)}^{2} d \underline{u}^{\prime} \int_{0}^{\underline{u}}\|\nabla \Psi\|_{\mathcal{L}_{(\mathrm{sc})}^{2}\left(u, \underline{u}^{\prime}\right)}^{2} d \underline{u}^{\prime}\right)^{1 / 2} \\
& \lesssim C \mathcal{R}_{0}^{1 / 2}\left(\mathcal{R}_{0}+\mathcal{R}_{1}\right)^{1 / 2} .
\end{aligned}
$$

We have thus established that

$$
\|\Theta\|_{\mathcal{L}_{(\mathrm{sc})}^{2}(u, \underline{u})} \lesssim \delta^{1 / 2} \Delta_{0}{ }^{(S)} \mathcal{O}_{1,2}+C \mathcal{R}_{0}^{1 / 2}\left(\mathcal{R}_{0}+\mathcal{R}_{1}\right)^{1 / 2}+\delta^{1 / 4} C^{2}
$$

We next estimate the $\Theta$ components which satisfy the $\nabla_{3}$ equation (6.22). The only terms which do not appear in (6.21) are of the form $\left(\operatorname{tr} \underline{\chi}_{0}\right) \nabla \psi$. Thus, exactly as before,

$$
\left\|\nabla_{3} \Theta\right\|_{\mathcal{L}_{(\mathrm{sc})}^{2}(S)} \lesssim\|\psi \Psi\|_{\mathcal{L}_{(\mathrm{sc})}^{2}(S)}+\left(1+\delta^{1 / 2} \Delta_{0}\right)\|\nabla \psi\|_{\mathcal{L}_{(\mathrm{sc})}^{2}(S)}+\delta^{1 / 4} C^{2}
$$

In view of Remark $6.5, \Psi \in\{\beta, \varrho, \sigma, \underline{\alpha}, \underline{\beta}\}$ and there are no double anomalous terms $\psi \cdot \Psi$. Thus, proceeding exactly as above,

$$
\begin{aligned}
\|\Theta\|_{\mathcal{L}_{(\mathrm{sc})}^{2}(u, \underline{u})} \lesssim\|\Theta\|_{\mathcal{L}_{(\mathrm{sc})}^{2}(0, \underline{u})}+\int_{0}^{u}\left\|\nabla_{3} \Theta\right\|_{\mathcal{L}_{(\mathrm{sc})}^{2}\left(u^{\prime}, \underline{u}\right)} d u^{\prime} \\
\lesssim \int_{0}^{u}\|\nabla \psi\|_{\mathcal{L}_{(\mathrm{sc})}^{2}\left(u^{\prime}, \underline{u}\right)} d u^{\prime}+\delta^{1 / 2} \Delta_{0}{ }^{(S)} \mathcal{O}_{1,2} \\
+C \delta^{1 / 4} \underline{\mathcal{R}}_{0}^{1 / 2}\left(\underline{\mathcal{R}}_{1}+\underline{\mathcal{R}}_{0}\right)^{1 / 2}+C^{2} \delta^{1 / 4}
\end{aligned}
$$

Combining this with (6.3), we deduce, for a constant $C=C\left(\mathcal{O}^{(0)}, \mathcal{R}, \underline{\mathcal{R}}\right)$ and a sufficiently small $\delta$, that

$$
\|\Theta\|_{\mathcal{L}_{(\mathrm{sc})}^{2}(u, \underline{u})} \lesssim C+\int_{0}^{u}\|\nabla \psi\|_{\mathcal{L}_{(\mathrm{sc})}^{2}\left(u^{\prime}, \underline{u}\right)} d u^{\prime}+\delta^{1 / 2} \Delta_{0} \mathcal{O}_{1} .
$$

It remains to discuss estimates for the Hodge systems (6.23). The following proposition will be needed.

Proposition 6.9. There exists a constant $C=C\left(\mathcal{O}^{(0)}, \mathcal{R}, \underline{\mathcal{R}}\right)$ such that, if $\delta$ is sufficiently small, the following estimates hold:

$$
\begin{aligned}
\|(\beta, \varrho, \sigma, \underline{\beta})\|_{\mathcal{L}_{(\mathrm{sc})}^{2}(S)} & \lesssim C, \\
\|K\|_{\mathcal{L}_{(\mathrm{sc})}^{2}(S)} & \lesssim C .
\end{aligned}
$$


In view of Proposition 4.11, we derive from (6.23) that

$$
\begin{aligned}
\|\nabla \psi\|_{\mathcal{L}_{(\mathrm{sc})}^{2}(S)} \lesssim \delta^{1 / 4}\|K\|_{\mathcal{L}_{(\mathrm{sc})}^{2}(S)}^{1 / 2}\|\psi\|_{\mathcal{L}_{(\mathrm{sc})}^{4}(S)}+\|\Theta\|_{\mathcal{L}_{(\mathrm{sc})}^{2}(S)} \\
+\|\Psi\|_{\mathcal{L}_{(\mathrm{sc})}^{2}(S)}+\left\|\psi_{g}\right\|_{\mathcal{L}_{(\mathrm{sc})}^{2}(S)}+\|\psi \psi\|_{\mathcal{L}_{(\mathrm{sc})}^{2}(S)}
\end{aligned}
$$

According to Proposition $6.9,\|K\|_{\mathcal{L}_{(\mathrm{sc})}^{2}(S)} \lesssim C$. Thus, even if the term $\|\psi\|_{\mathcal{L}_{\text {(sc) }}^{2}(S)}$ multiplying $\|K\|_{\mathcal{L}_{(\mathrm{sc})}^{2}(S)}$ is anomalous, $\left({ }^{16}\right)$ i.e. $\|\psi\|_{\mathcal{L}_{(\mathrm{sc})}^{4}(u, \underline{u})} \lesssim \delta^{-1 / 4(S)} \mathcal{O}_{0,4} \lesssim C \delta^{-1 / 4}$, we deduce that, for some $C=C\left(\mathcal{O}^{(0)}, \mathcal{R}, \underline{\mathcal{R}}\right)$,

$$
\delta^{1 / 4}\|K\|_{\mathcal{L}_{(\mathrm{sc})}^{2}(S)}^{1 / 2}\|\psi\|_{\mathcal{L}_{(\mathrm{sc})}^{4}(S)} \lesssim C .
$$

Also, since $\|\Psi\|_{\mathcal{L}_{(\mathrm{sc})}^{2}(S)} \lesssim C$ for $\Psi \in\{\beta, \varrho, \sigma, \underline{\beta}\}$ and $\left\|\psi_{g}\right\|_{\mathcal{L}_{(\mathrm{sc})}^{2}(S)} \lesssim \mathcal{O}_{0}\left[\psi_{g}\right] \lesssim C$, we deduce that

$$
\|\nabla \psi\|_{\mathcal{L}_{(\mathrm{sc})}^{2}(S)} \lesssim C+\|\Theta\|_{\mathcal{L}_{(\mathrm{sc})}^{2}(S)}+\|\psi \psi\|_{\mathcal{L}_{(\mathrm{sc})}^{2}(S)} .
$$

Among the remaining quadratic terms $\|\psi \psi\|_{\mathcal{L}_{(\mathrm{sc})}^{2}(S)}$, we can have terms such as $\hat{\chi} \underline{\hat{\chi}}$, in which both factors are anomalous. $\left({ }^{17}\right)$ For such terms,

$$
\|\psi \psi\|_{\mathcal{L}_{(\mathrm{sc})}^{2}(S)} \lesssim \delta^{1 / 2}\|\psi\|_{\mathcal{L}_{(\mathrm{sc})}^{4}(S)}\|\psi\|_{\mathcal{L}_{(\mathrm{sc})}^{4}(S)} \lesssim C^{2}
$$

Henceforth,

$$
\|\nabla \psi\|_{\mathcal{L}_{(\mathrm{sc})}^{2}(S)} \lesssim C^{2}+\|\Theta\|_{\mathcal{L}_{(\mathrm{sc})}^{2}(S)}
$$

Combining this with (6.24), we deduce that

$$
\|\nabla \psi\|_{\mathcal{L}_{(\mathrm{sc})}^{2}\left(S_{u, \underline{u}}\right)} \lesssim C^{2}+\int_{0}^{u}\|\nabla \psi\|_{\mathcal{L}_{(\mathrm{sc})}^{2}\left(S_{u^{\prime}, \underline{u}}\right)} d u^{\prime}+\delta^{1 / 2} \Delta_{0} \mathcal{O}_{1}
$$

from which, by Grönwall's inequality,

$$
\|\nabla \psi\|_{\mathcal{L}_{(\mathrm{sc})}^{2}\left(S_{u, \underline{u}}\right)} \lesssim C^{2}+\delta^{1 / 2} \Delta_{0}{ }^{(S)} \mathcal{O}_{1,2}
$$

and thus

$$
{ }^{(S)} \mathcal{O}_{1,2}+\|\Theta\|_{\mathcal{L}_{(\mathrm{sc})}^{2}(S)} \lesssim C^{2}
$$

as desired. We summarize the results in the following proposition.

Proposition 6.10. Consider systems of the form (6.1)-(6.3) satisfying the properties discussed in Remarks 6.3-6.7 above. There exists a constant $C=C\left(\mathcal{O}^{(0)}, \mathcal{R}, \underline{\mathcal{R}}\right)$ such that

$$
\|\Theta\|_{\mathcal{L}_{(\mathrm{sc})}^{2}(S)}+{ }^{(S)} \mathcal{O}_{1,2} \lesssim C
$$

$\left.{ }^{(16}\right)$ This situation occurs only for the Hodge system $\operatorname{div} \hat{\chi}$, see $(6.4)$, since $\mathcal{O}_{0}[\widehat{\chi}]$ is anomalous.

(17) In fact $\widehat{\chi} \underline{\hat{\chi}}$ appears in the Hodge systems for $\eta$ and $\underline{\eta}$, see formulas (6.12) and (6.13). 


\subsection{Curvature estimates}

In this subsection we prove Proposition 6.9 concerning $\mathcal{L}_{(\mathrm{sc})}^{2}(S)$ estimates for the curvature components $\beta, \varrho, \sigma$ and $\underline{\beta}$. We also provide estimates for $\alpha$ and $\underline{\alpha}$ which will be needed later. Recall the Bianchi identities

$$
\begin{aligned}
\nabla_{4} \beta+2(\operatorname{tr} \chi) \beta & =\operatorname{div} \alpha-2 \omega \beta-(2 \zeta+\underline{\eta}) \alpha \\
\nabla_{4} \varrho+\frac{3}{2}(\operatorname{tr} \chi) \varrho & =\operatorname{div} \beta+\frac{1}{2} \underline{\widehat{\chi}} \alpha-\zeta \beta-2 \underline{\eta} \beta \\
\nabla_{4} \sigma+\frac{3}{2}(\operatorname{tr} \chi) \sigma & =-\operatorname{div}^{*} \beta+\frac{1}{2} \underline{\widehat{\chi}}^{*} \alpha-\zeta^{*} \beta-2 \underline{\eta}^{*} \beta \\
\nabla_{4} \underline{\beta}+(\operatorname{tr} \chi) \underline{\beta} & =-\nabla \varrho+{ }^{*} \nabla \sigma+2 \omega \underline{\beta}+2 \underline{\hat{\chi}} \beta-3\left(\underline{\eta} \varrho-{ }^{*} \underline{\eta} \sigma\right) .
\end{aligned}
$$

Thus $\beta, \varrho, \sigma$ and $\underline{\beta}$ satisfy equations of the form

$$
\nabla_{4} \Psi^{(s)}=\nabla \Psi^{(s+1 / 2)}+\sum_{s_{1}+s_{2}=s+1} \psi^{\left(s_{1}\right)} \Psi^{\left(s_{2}\right)}
$$

Among the curvature terms on the right, we have to pay special attention to multiples of the curvature term $\alpha$ with signature 2 . We write schematically

$$
\nabla_{4} \Psi_{g}=\nabla \Psi+\psi \Psi
$$

with $\Psi_{g} \in\{\beta, \varrho, \sigma, \underline{\beta}\}$, while $\Psi \in\{\alpha, \beta, \varrho, \sigma, \underline{\beta}\}$.

Thus,

$$
\left\|\nabla_{4} \Psi_{g}\right\|_{\mathcal{L}_{(\mathrm{sc})}^{2}(S)} \lesssim\|\nabla \Psi\|_{\mathcal{L}_{(\mathrm{sc})}^{2}(S)}+\|\alpha \psi\|_{\mathcal{L}_{(\mathrm{sc})}^{2}(S)}+\delta^{1 / 2}\|\psi\|_{\mathcal{L}_{(\mathrm{sc})}^{\infty}}\left\|\Psi_{g}\right\|_{\mathcal{L}_{(\mathrm{sc})}^{2}(S)}
$$

Now, as in the estimates for $\Theta$ in the previous section, the worst case scenario estimate for $\|\alpha \psi\|_{\mathcal{L}_{(\mathrm{sc})}^{2}(S)}$, for anomalous $\psi$, has the form

$$
\|\psi \alpha\|_{\mathcal{L}_{(\mathrm{sc})}^{2}(S)} \lesssim C \delta^{1 / 4}\left(\|\nabla \alpha\|_{\mathcal{L}_{(\mathrm{sc})}^{2}(S)}^{1 / 2}\|\alpha\|_{\mathcal{L}_{(\mathrm{sc})}^{2}(S)}^{1 / 2}+\delta^{1 / 4}\|\alpha\|_{\mathcal{L}_{(\mathrm{sc})}^{2}(S)}\right) .
$$

We deduce that

$$
\begin{aligned}
\left\|\nabla_{4} \Psi_{g}\right\|_{\mathcal{L}_{(\mathrm{sc})}^{2}(S) \lesssim} & \|\nabla \Psi\|_{\mathcal{L}_{(\mathrm{sc})}^{2}(S)}+\delta^{1 / 2} \Delta_{0}\left\|\Psi_{g}\right\|_{\mathcal{L}_{(\mathrm{sc})}^{2}(S)} \\
& +C \delta^{1 / 4}\left(\|\nabla \alpha\|_{\mathcal{L}_{(\mathrm{sc})}^{2}(S)}^{1 / 2}\|\alpha\|_{\mathcal{L}_{(\mathrm{sc})}^{2}(S)}^{2}+\delta^{1 / 4}\|\alpha\|_{\mathcal{L}_{(\mathrm{sc})}^{2}(S)}\right)
\end{aligned}
$$

from which

$$
\left\|\Psi_{g}\right\|_{\mathcal{L}_{(\mathrm{sc})}^{2}(u, \underline{u})} \lesssim\left\|\Psi_{g}\right\|_{\mathcal{L}_{(\mathrm{sc})}^{2}(u, 0)}+\mathcal{R}_{1}+\delta^{1 / 2} \Delta_{0} \mathcal{R}_{0}+C \mathcal{R}_{0}^{1 / 2}[\alpha] \mathcal{R}_{1}^{1 / 2}[\alpha]+C \mathcal{R}_{0}[\alpha]
$$


Thus, since the initial data $\left\|\Psi_{g}\right\|_{\mathcal{L}_{(\mathrm{sc})}^{2}(u, 0)}$ is trivial,

$$
\left\|\Psi_{g}\right\|_{\mathcal{L}_{(\mathrm{sc})}^{2}(u, \underline{u})} \lesssim \mathcal{R}_{1}+\delta^{1 / 2} \Delta_{0} \mathcal{R}_{0}+C \mathcal{R}_{0}^{1 / 2}[\alpha] \mathcal{R}_{1}^{1 / 2}[\alpha]+C \mathcal{R}_{0}[\alpha]
$$

or, with a new constant $C=C\left(\mathcal{O}^{(0)}, \mathcal{R}, \underline{\mathcal{R}}\right)$,

$$
\left\|\Psi_{g}\right\|_{\mathcal{L}_{(\mathrm{sc})}^{2}(u, \underline{u})} \lesssim C
$$

as desired.

It remains to estimate the $\mathcal{L}_{(\mathrm{sc})}^{2}(S)$ norm of the Gauss curvature

$$
K=-\varrho+\frac{1}{2} \widehat{\chi} \underline{\hat{\chi}}-\frac{1}{4}(\operatorname{tr} \chi) \operatorname{tr} \underline{\chi}=\varrho+\frac{1}{2} \widehat{\chi} \underline{\widehat{\chi}}-\frac{1}{4}(\operatorname{tr} \chi) \operatorname{tr} \underline{\chi}_{0}-\frac{1}{4}(\operatorname{tr} \chi) \widetilde{\operatorname{tr}} \underline{\chi} .
$$

Thus,

$$
\begin{aligned}
\|K\|_{\mathcal{L}_{(\mathrm{sc})}^{2}(S)} & \lesssim\|\varrho\|_{\mathcal{L}_{(\mathrm{sc})}^{2}(S)}+\delta^{1 / 2}\|\widehat{\chi}\|_{\mathcal{L}_{(\mathrm{sc})}^{4}(S)}\|\underline{\hat{\chi}}\|_{\mathcal{L}_{(\mathrm{sc})}^{4}(S)}+\|\operatorname{tr} \chi\|_{\mathcal{L}_{(\mathrm{sc})}^{2}}+\delta^{1 / 2} \Delta_{0}\|\widetilde{\operatorname{tr} \underline{\chi}}\|_{\mathcal{L}_{(\mathrm{sc})}^{2}} \\
& \lesssim C+\delta^{1 / 2} \Delta_{0} \mathcal{R}_{0},
\end{aligned}
$$

from which the desired estimate follows:

$$
\|K\|_{\mathcal{L}_{(\mathrm{sc})}^{2}(S)} \lesssim C\left(\mathcal{O}^{(0)}, \mathcal{R}, \underline{\mathcal{R}}\right)
$$

In the next proposition we derive estimates for the remaining curvature components.

Proposition 6.11. There exists a constant $C=C\left(\mathcal{O}^{(0)}, \mathcal{R}, \underline{\mathcal{R}}\right)$ such that, for $\delta^{1 / 2} \Delta_{0}$ sufficiently small,

$$
\|\alpha\|_{\mathcal{L}_{(\mathrm{sc})}^{2}(S)} \leqslant C \delta^{-1 / 2} \quad \text { and } \quad\|\underline{\alpha}\|_{\mathcal{L}_{(\mathrm{sc})}^{2}(S)} \leqslant C .
$$

Proof. To prove the estimate for $\alpha$ we use the Bianchi equation for $\nabla_{3} \alpha$, which can be written schematically in the form

$$
\nabla_{3} \alpha=\left(\operatorname{tr} \underline{\chi}_{0}\right) \alpha+\psi \alpha+\nabla \Psi+\psi \Psi
$$

with $\Psi$ from the set not containing $\alpha$. We therefore obtain

$$
\begin{gathered}
\|\alpha\|_{\mathcal{L}_{(\mathrm{sc})}^{2}\left(S_{u, \underline{u}}\right)} \lesssim\|\alpha\|_{\mathcal{L}_{(\mathrm{sc})}^{2}\left(S_{0, \underline{u}}\right)}+\left(1+\delta^{1 / 2} \Delta_{0}\right) \int_{0}^{u}\|\alpha\|_{\mathcal{L}_{(\mathrm{sc})}^{2}\left(S_{u^{\prime}, \underline{u}}\right)} d u^{\prime} \\
+\underline{\mathcal{R}}_{1}+\delta^{1 / 2} \Delta_{0}\|\Psi\|_{\mathcal{L}_{(\mathrm{sc})}^{2}\left(\underline{H}_{\underline{u}}\right)} .
\end{gathered}
$$

In the worst case when $\Psi=\beta$, which is anomalous, we have $\|\Psi\|_{\mathcal{L}_{(\mathrm{sc})}^{2}\left(\underline{H}_{\underline{u}}\right)} \lesssim \delta^{-1 / 2} \underline{\mathcal{R}}_{0}$. Thus, by Grönwall's inequality,

$$
\|\alpha\|_{\mathcal{L}_{(\mathrm{sc})}^{2}\left(S_{u, \underline{u}}\right)} \lesssim\|\alpha\|_{\mathcal{L}_{(\mathrm{sc})}^{2}\left(S_{0, \underline{u}}\right)}+\underline{\mathcal{R}} .
$$


Similarly, the equation for $\nabla_{4} \underline{\alpha}$ has the form

$$
\nabla_{4} \underline{\alpha}=\nabla \Psi+\psi \Psi
$$

where the curvature term in $\nabla \Psi$ is not $\underline{\alpha}$ and $\Psi \neq \alpha$ in the non-linear term. Therefore, using the triviality of initial data,

$$
\|\underline{\alpha}\|_{\mathcal{L}_{(\mathrm{sc})}^{2}(u, \underline{u})} \lesssim \mathcal{R}_{1}+\delta^{1 / 2} \Delta_{0} \int_{0}^{u}\left(\|\underline{\alpha}\|_{\mathcal{L}_{(\mathrm{sc})}^{2}\left(u^{\prime}, \underline{u}\right)}+\left\|\Psi_{g}\right\|_{\mathcal{L}_{(\mathrm{sc})}^{2}\left(u^{\prime}, \underline{u}\right)}\right) d u^{\prime},
$$

with $\Psi_{g} \in\{\varrho, \sigma, \underline{\beta}\}$. The result then easily follows by Grönwall's inequality and the $\mathcal{L}_{(\mathrm{sc})}^{2}(H)$ curvature bounds for $\Psi_{g}$.

\section{Second angular derivative estimates for the Ricci coefficients}

To obtain second angular derivative estimates for the Ricci coefficients, we differentiate (6.1)-(6.3) with respect to $\nabla$.

\subsection{Basic equations}

Based on the experience with the first derivative estimates, we expect that the $\nabla_{3}$ equation for $\nabla \Theta$ is slightly more challenging as it contains a lot more $\operatorname{tr} \underline{\chi}$ terms. Thus, differentiating (6.22), we get

$$
\begin{gathered}
\nabla_{3} \nabla \Theta=\left(\operatorname{tr} \underline{\chi}_{0}\right)\left(\nabla \Theta+\nabla \Psi+\nabla^{2} \psi\right)+\psi\left(\nabla \Theta+\nabla \Psi+\nabla^{2} \psi\right)+(\nabla \psi)(\Theta+\Psi+\nabla \psi) \\
+\left(\operatorname{tr} \underline{\chi}_{0}\right) \psi \nabla \psi+\psi \psi \nabla \psi+\left[\nabla_{3}, \nabla\right] \Theta
\end{gathered}
$$

According to commutation formulas of Lemma 3.1, we write symbolically

$$
\begin{aligned}
{\left[\nabla_{3}, \nabla\right] \Theta } & =(\operatorname{tr} \underline{\chi}) \nabla \Theta+\underline{\hat{\chi}} \nabla \Theta+\Psi \Theta+(\operatorname{tr} \underline{\chi}) \psi \Theta+\psi \psi \Theta+\psi \nabla_{3} \Theta \\
& =\left(\operatorname{tr} \underline{\chi}_{0}\right) \nabla \Theta+\psi \nabla \Theta+\Psi \Theta+\left(\operatorname{tr} \underline{\chi}_{0}\right) \psi \Theta+\psi \psi \Theta+\psi \nabla_{3} \Theta .
\end{aligned}
$$

Hence,

$$
\begin{gathered}
\nabla_{3} \nabla \Theta=\left(\operatorname{tr} \underline{\chi}_{0}\right)\left(\nabla \Theta+\nabla \Psi+\nabla^{2} \psi\right)+\psi\left(\nabla \Theta+\nabla \Psi+\nabla^{2} \psi\right)+(\nabla \psi)(\Theta+\Psi+\nabla \psi) \\
+\Theta \Psi+\left(\operatorname{tr} \underline{\chi}_{0}\right)(\psi \nabla \psi+\psi \Theta)+\psi\left(\psi \nabla \psi+\psi \Theta+\nabla_{3} \Theta\right)
\end{gathered}
$$

Ignoring the term of the form $\left(\operatorname{tr} \underline{\chi}_{0}\right) \nabla \Theta$ which can be easily eliminated by Grönwall's inequality, and observing that $\Theta$, resp. $\nabla \Theta$, on the left can be expressed in terms of $\nabla \psi$ and $\Psi$, resp. $\nabla^{2} \psi$ and $\nabla \Psi$, we write

$$
\begin{aligned}
\nabla_{3} \nabla \Theta= & \left(\operatorname{tr} \underline{\chi}_{0}+\psi\right)\left(\nabla \Psi+\nabla^{2} \psi\right)+\left(\operatorname{tr} \underline{\chi}_{0}\right)(\psi \nabla \psi+\psi \Theta)+(\nabla \psi)(\Psi+\nabla \psi) \\
& +\Theta \Psi+\psi\left(\psi \nabla \psi+\psi \Theta+\nabla_{3} \Theta\right) \\
= & F_{1}+F_{2}+F_{3}+F_{4}+F_{5} .
\end{aligned}
$$


Similarly,

$$
\nabla_{4} \nabla \Theta=\psi\left(\nabla \Psi+\nabla^{2} \psi\right)+(\nabla \psi)(\Psi+\nabla \psi)+\Theta \Psi+\psi \psi \nabla \psi+\left[\nabla_{4}, \nabla\right] \Theta
$$

and

$$
\left[\nabla_{4}, \nabla\right] \Theta=\psi \nabla \Theta+\Psi \Theta+\psi \psi \Theta+\psi \nabla_{4} \Theta
$$

so that

$$
\begin{aligned}
\nabla_{4} \nabla \Theta & =\psi\left(\nabla \Psi+\nabla^{2} \psi\right)+(\nabla \psi)(\Psi+\nabla \psi)+\psi\left(\psi \nabla \psi+\psi \Theta+\nabla_{4} \Theta\right) \\
& =G_{1}+G_{2}+G_{3}
\end{aligned}
$$

Equations (7.1) and (7.2) will be combined with the differentiated Hodge system for $\psi$ in (6.23):

$$
\mathcal{D}^{*} \mathcal{D} \psi=\mathcal{D}^{*}\left(\Theta+\Psi+\psi \psi+\left(\operatorname{tr} \underline{\chi}_{0}\right) \psi\right),
$$

which can be schematically written in the form

$$
\Delta \psi=K \psi+\nabla \Theta+\nabla \Psi+(\nabla \psi) \psi+\left(\operatorname{tr} \underline{\chi}_{0}\right) \nabla \psi
$$

\subsection{Estimates for $\nabla \Theta$ and $\nabla^{2} \psi$}

We now collect estimates for the terms on the right-hand side of the transport equations (7.1) and (7.2):

$$
\begin{aligned}
& \left\|F_{1}\right\|_{\mathcal{L}_{\text {(sc) }}^{2}(S)} \lesssim\left(1+\delta^{1 / 2} \Delta_{0}\right)\left(\left\|\nabla^{2} \psi\right\|_{\mathcal{L}_{(\mathrm{sc})}^{2}(S)}+\|\nabla \Psi\|_{\mathcal{L}_{(\mathrm{sc})}^{2}(S)}\right), \\
& \left\|F_{2}\right\|_{\mathcal{L}_{\text {(sc) }}^{2}(S)} \lesssim \delta^{1 / 2} \Delta_{0}\left(\|\Theta\|_{\mathcal{L}_{(\mathrm{sc})}^{2}(S)}+\|\nabla \psi\|_{\mathcal{L}_{(\mathrm{sc})}^{2}(S)}\right), \\
& \left\|F_{3}\right\|_{\mathcal{L}_{(\mathrm{sc})}^{2}(S)} \lesssim \delta^{1 / 2}\|\nabla \psi\|_{\mathcal{L}_{(\mathrm{sc})}^{4}(S)}\left(\|\nabla \psi\|_{\mathcal{L}_{(\mathrm{sc})}^{4}(S)}+\|\Psi\|_{\mathcal{L}_{(\mathrm{sc})}^{4}(S)}\right), \\
& \left\|F_{4}\right\|_{\mathcal{L}_{(\mathrm{sc})}^{2}(S)} \lesssim \delta^{1 / 2}\|\Theta\|_{\mathcal{L}_{(\mathrm{sc})}^{4}(S)}\|\Psi\|_{\mathcal{L}_{(\mathrm{sc})}^{4}(S)}, \\
& \left\|F_{5}\right\|_{\mathcal{L}_{\text {(sc) }}^{2}(S)} \lesssim \delta^{1 / 2} \Delta_{0}\left(\delta^{1 / 2} \Delta_{0}\|(\nabla \psi, \Theta)\|_{\mathcal{L}_{(\mathrm{sc})}^{2}(S)}+\left\|\nabla_{3} \Theta\right\|_{\mathcal{L}_{\text {(sc) }}^{2}(S)}\right) .
\end{aligned}
$$

Similarly,

$$
\begin{aligned}
\left\|G_{1}\right\|_{\mathcal{L}_{\text {(sc) }}^{2}(S)} & \lesssim \delta^{1 / 2} \Delta_{0}\left(\|\nabla \Theta\|_{\mathcal{L}_{\text {(sc) }}^{2}(S)}+\left\|\nabla^{2} \psi\right\|_{\mathcal{L}_{\text {(sc) }}^{2}(S)}+\|\nabla \Psi\|_{\mathcal{L}_{\text {(sc) }}^{2}(S)}\right) \\
\left\|G_{2}\right\|_{\mathcal{L}_{\text {(sc) }}^{2}(S)} & \lesssim \delta^{1 / 2}\|\nabla \psi\|_{\mathcal{L}_{\text {(sc) }}^{4}(S)}\left(\|\nabla \psi\|_{\mathcal{L}_{\text {(sc) }}^{4}(S)}+\|\Psi\|_{\mathcal{L}_{\text {(sc) }}^{4}(S)}\right) \\
\left\|G_{3}\right\|_{\mathcal{L}_{\text {(sc) }}^{2}(S)} & \lesssim \delta^{1 / 2} \Delta_{0}\left(\delta^{1 / 2} \Delta_{0}\|(\nabla \psi, \Theta)\|_{\mathcal{L}_{(\mathrm{sc})}^{2}(S)}+\left\|\nabla_{4} \Theta\right\|_{\mathcal{L}_{(\mathrm{sc})}^{2}(S)}\right)
\end{aligned}
$$


We note that the curvature terms $\Psi$ present in the $F$ terms belong to the admissible set $\{\beta, \varrho, \sigma, \underline{\alpha}, \underline{\beta}\}$, while the curvature terms $\Psi$ appearing in the $G$ terms belong to the set $\{\alpha, \beta, \varrho, \sigma, \underline{\beta}\}$. We also recall that, according to the ${ }^{(S)} \mathcal{O}_{1,2}$ estimates and their consequences proved in the previous section,

$$
\|\nabla \psi\|_{L^{2}(S)}+\|\Theta\|_{L^{2}(S)}+\left\|\nabla_{4} \Theta\right\|_{L^{2}(H)}+\left\|\nabla_{3} \Theta\right\|_{L^{2}(\underline{H})} \leqslant C .
$$

Using the $L^{4}$ interpolation estimate from (4.19), which imply that

$$
\begin{aligned}
\|\nabla \psi\|_{\mathcal{L}_{(\mathrm{sc})}^{4}(S)} & \lesssim\|\nabla \psi\|_{\mathcal{L}_{(\mathrm{sc})}^{2}(S)}^{1 / 2}\left\|\nabla^{2} \psi\right\|_{\mathcal{L}_{(\mathrm{sc})}^{2}(S)}^{1 / 2}+\delta^{1 / 4}\|\nabla \psi\|_{\mathcal{L}_{(\mathrm{sc})}^{2}(S)} \lesssim C\left\|\nabla^{2} \psi\right\|_{\mathcal{L}_{(\mathrm{sc})}^{2}(S)}^{1 / 2}+\delta^{1 / 4} C, \\
\|\Theta\|_{\mathcal{L}_{(\mathrm{sc})}^{4}(S)} & \lesssim\|\Theta\|_{\mathcal{L}_{(\mathrm{sc})}^{2}(S)}^{1 / 2}\|\nabla \Theta\|_{\mathcal{L}_{(\mathrm{sc})}^{2}(S)}^{1 / 2}+\delta^{1 / 4}\|\Theta\|_{\mathcal{L}_{(\mathrm{sc})}^{2}(S)} \lesssim C\|\nabla \Theta\|_{\mathcal{L}_{(\mathrm{sc})}^{2}(S)}^{1 / 2}+\delta^{1 / 4} C, \\
\|\Psi\|_{\mathcal{L}_{(\mathrm{sc})}^{4}(S)} & \lesssim\|\Psi\|_{\mathcal{L}_{(\mathrm{sc})}^{2}(S)}^{1 / 2}\|\nabla \Psi\|_{\mathcal{L}_{(\mathrm{sc})}^{2}(S)}^{1 / 2}+\delta^{1 / 4}\|\Psi\|_{\mathcal{L}_{(\mathrm{sc})}^{2}(S)},
\end{aligned}
$$

we obtain, for $\delta^{1 / 2} \Delta_{0}$ sufficiently small, that

$$
\begin{aligned}
& \|\nabla \Theta\|_{\mathcal{L}_{\text {(sc) }}^{2}(u, \underline{u})} \lesssim\|\nabla \Theta\|_{\mathcal{L}_{\text {(sc) }}^{2}(0, \underline{u})}+\int_{0}^{u}\left\|\nabla_{3} \nabla \Theta\right\|_{\mathcal{L}_{\text {(sc) }}^{2}\left(u^{\prime}, \underline{u}\right)} d u^{\prime} \\
& \lesssim\|\nabla \Theta\|_{\mathcal{L}_{\text {(sc) }}^{2}(0, \underline{u})}+C \int_{0}^{u}\left(\left\|\nabla^{2} \psi\right\|_{\mathcal{L}_{\text {(sc) }}^{2}}+\|\nabla \Psi\|_{\mathcal{L}_{\text {(sc) }}^{2}}\right) d u^{\prime} \\
& +\delta^{1 / 2} C \int_{0}^{u}\left(\left\|\nabla^{2} \psi\right\|_{\mathcal{L}_{(\mathrm{sc})}^{2}(S)}^{1 / 2}\|\Psi\|_{\mathcal{L}_{(\mathrm{sc})}^{2}(S)}^{1 / 2}\|\nabla \Psi\|_{\mathcal{L}_{(\mathrm{sc})}^{2}(S)}^{1 / 2}\right. \\
& \left.+\delta^{1 / 4}\left\|\nabla^{2} \psi\right\|_{\mathcal{L}_{(\mathrm{sc})}^{2}(S)}^{1 / 2}\|\Psi\|_{\mathcal{L}_{(\mathrm{sc})}^{2}(S)}^{1 / 2}\right) d u^{\prime} \\
& +\delta^{1 / 2} C \int_{0}^{u}\left(\|\nabla \Theta\|_{\mathcal{L}_{(\mathrm{sc})}^{2}(S)}^{1 / 2}\|\Psi\|_{\mathcal{L}_{(\mathrm{sc})}^{2}(S)}^{1 / 2}\|\nabla \Psi\|_{\mathcal{L}_{(\mathrm{sc})}^{2}(S)}^{1 / 2}\right. \\
& \left.+\delta^{1 / 4}\|\nabla \Theta\|_{\mathcal{L}_{(\mathrm{sc})}^{2}(S)}^{1 / 2}\|\Psi\|_{\mathcal{L}_{(\mathrm{sc})}^{2}(S)}\right) d u^{\prime}+\delta^{1 / 2} C .
\end{aligned}
$$

We kept track of the terms containing $\|\Psi\|_{\mathcal{L}_{(\mathrm{sc})}^{2}(S)}$ as they may lead to the potentially anomalous norm $\|\Psi\|_{\mathcal{L}_{(\mathrm{sc})}^{2}(\underline{H})}$ in the case when $\Psi=\beta$. However, even in that case

$$
\|\Psi\|_{\mathcal{L}_{(\mathrm{sc})}^{2}(\underline{H})} \lesssim \delta^{-1 / 2} \underline{\mathcal{R}}_{0} .
$$

By Grönwall's inequality, and recalling the definition $\left({ }^{18}\right)$ of $\underline{\mathcal{R}}_{1}$, we have

$$
\|\nabla \Theta\|_{\mathcal{L}_{\text {(sc) }}^{2}(u, \underline{u})} \lesssim\|\nabla \Theta\|_{\mathcal{L}_{(\mathrm{sc})}^{2}(0, \underline{u})}+C \int_{0}^{u}\left\|\nabla^{2} \psi\right\|_{\mathcal{L}_{\text {(sc) }}^{2}\left(u^{\prime}, \underline{u}\right)} d u^{\prime}+C \underline{\mathcal{R}}_{1}
$$

In view of the estimates for the $G$ terms, we similarly obtain

$$
\|\nabla \Theta\|_{\mathcal{L}_{\text {(sc) }}^{2}(u, \underline{u})} \lesssim\|\nabla \Theta\|_{\mathcal{L}_{\text {(sc) }}^{2}(u, 0)}+C \int_{0}^{\underline{u}}\left\|\nabla^{2} \psi\right\|_{\mathcal{L}_{\text {(sc) }}^{2}\left(u, \underline{u}^{\prime}\right)} d \underline{u}^{\prime}+C \mathcal{R}_{1}
$$

\footnotetext{
$\left({ }^{18}\right)$ Mote again that $\underline{\alpha}$ does not appear among the $\Psi$ 's.
} 
We now couple this with the second derivative estimates for the Hodge system

$$
D \psi=\Theta+\Psi+\left(\operatorname{tr} \underline{\chi}_{0}\right) \psi+\psi \psi .
$$

Using Proposition 4.12, we deduce that

$$
\begin{aligned}
\left\|\nabla^{2} \psi\right\|_{\mathcal{L}_{(\mathrm{sc})}^{2}(S)} \lesssim & \delta^{1 / 2}\|K\|_{\mathcal{L}_{(\mathrm{sc})}^{2}(S)}\|\psi\|_{\mathcal{L}_{(\mathrm{sc})}^{\infty}(S)}+\delta^{1 / 4}\|K\|_{\mathcal{L}_{(\mathrm{sc})}^{2}(S)}^{1 / 2}\|\nabla \psi\|_{\mathcal{L}_{(\mathrm{sc})}^{4}(S)} \\
& +\|\nabla \Theta\|_{\mathcal{L}_{(\mathrm{sc})}^{2}(S)}+\|\nabla \Psi\|_{\mathcal{L}_{(\mathrm{sc})}^{2}(S)}+\left\|\left(\operatorname{tr} \underline{\chi}_{0}\right) \nabla \psi\right\|_{\mathcal{L}_{(\mathrm{sc})}^{2}(S)}+\|\psi \nabla \psi\|_{\mathcal{L}_{(\mathrm{sc})}^{2}(S)} .
\end{aligned}
$$

By Proposition $6.9,\|K\|_{\mathcal{L}_{(\mathrm{sc})}^{2}(S)} \lesssim C$ with a constant $C=C\left(\mathcal{O}^{(0)}, \mathcal{R}, \underline{\mathcal{R}}\right)$. Therefore,

$$
\begin{aligned}
\left\|\nabla^{2} \psi\right\|_{\mathcal{L}_{(\mathrm{sc})}^{2}(S)} \lesssim \delta^{1 / 2} C \Delta_{0}+\delta^{1 / 4} C\left(\left\|\nabla^{2} \psi\right\|_{\mathcal{L}_{(\mathrm{sc})}^{2}(S)}^{1 / 2}\|\nabla \psi\|_{\mathcal{L}_{(\mathrm{sc})}^{2}(S)}^{1 / 2}+\delta^{1 / 4}\|\nabla \psi\|_{\mathcal{L}_{(\mathrm{sc})}^{2}(S)}\right) \\
+\|\nabla \Theta\|_{\mathcal{L}_{(\mathrm{sc})}^{2}(S)}+\|\nabla \Psi\|_{\mathcal{L}_{(\mathrm{sc})}^{2}(S)}+\|\nabla \psi\|_{\mathcal{L}_{(\mathrm{sc})}^{2}(S)}+\delta^{1 / 2} C \Delta_{0}\|\nabla \psi\|_{\mathcal{L}_{(\mathrm{sc})}^{2}(S)}
\end{aligned}
$$

Using the Cauchy-Schwarz inequality and the boundedness of the ${ }^{(S)} \mathcal{O}_{1,2}$ norm, we get

$$
\left\|\nabla^{2} \psi\right\|_{\mathcal{L}_{(\mathrm{sc})}^{2}(S)} \lesssim C+\|\nabla \Theta\|_{\mathcal{L}_{(\mathrm{sc})}^{2}(S)}+\|\nabla \Psi\|_{\mathcal{L}_{(\mathrm{sc})}^{2}(S)} .
$$

We note that the curvature terms $\Psi$ involved in the above inequality belong to the set $\{\beta, \varrho, \sigma, \beta\}$. In particular,

$$
\|\nabla \Psi\|_{\mathcal{L}_{(\mathrm{sc})}^{2}(H)} \lesssim \mathcal{R}_{1} \quad \text { and } \quad\|\nabla \Psi\|_{\mathcal{L}_{(\mathrm{sc})}^{2}(\underline{H})} \lesssim \underline{\mathcal{R}}_{1}
$$

Therefore, substituting the estimate for $\left\|\nabla^{2} \psi\right\|_{\mathcal{L}_{(\mathrm{sc})}^{2}(S)}$ into (7.3) and (7.4), and using Grönwall's inequality, we obtain that

$$
\begin{aligned}
\|\nabla \Theta\|_{\mathcal{L}_{(\mathrm{sc})}^{2}(u, \underline{u})} & \lesssim\|\nabla \Theta\|_{\mathcal{L}_{(\mathrm{sc})}^{2}(0, \underline{u})}+C \underline{\mathcal{R}}_{1}, \\
\|\nabla \Theta\|_{\mathcal{L}_{(\mathrm{sc})}^{2}(u, \underline{u})} & \lesssim\|\nabla \Theta\|_{\mathcal{L}_{(\mathrm{sc})}^{2}(u, 0)}+C \mathcal{R}_{1} .
\end{aligned}
$$

This, together with (7.5), in turn implies the following result.

Proposition 7.1. There exists a constant $C=C\left(\mathcal{O}^{(0)}, \mathcal{R}, \underline{\mathcal{R}}\right)$ such that all second derivatives $\nabla^{2} \psi$ of the Ricci coefficients

$$
\psi \in\{\operatorname{tr} \chi, \widehat{\chi}, \omega, \eta, \widetilde{\operatorname{tr}} \underline{\chi}, \underline{\chi}, \underline{\omega}, \underline{\eta}\}
$$

and the first derivatives of the quantities

$$
\Theta \in\left\{\nabla \operatorname{tr} \chi, \nabla \operatorname{tr} \underline{\chi}, \operatorname{div} \eta+\varrho, \operatorname{div} \underline{\eta}+\varrho, \nabla \omega+{ }^{*} \nabla \omega^{\dagger}-\frac{1}{2} \beta,-\nabla \omega+{ }^{*} \nabla \omega^{\dagger}-\frac{1}{2} \underline{\beta}\right\}
$$

satisfy

$$
\|\nabla \Theta\|_{\mathcal{L}_{(\mathrm{sc})}^{2}(u, \underline{u})}+\left\|\nabla^{2} \psi\right\|_{\mathcal{L}_{(\mathrm{sc})}^{2}\left(H_{u}\right)}+\left\|\nabla^{2} \psi\right\|_{\mathcal{L}_{(\mathrm{sc})}^{2}\left(H_{\underline{u}}\right)} \lesssim C .
$$




\section{3. ${ }^{(S)} \mathcal{O}_{1,4}$ estimates}

Proposition 7.1, together with Corollary 4.8, has the following consequence.

COROLlaRY 7.2. There exists a constant $C=C\left(\mathcal{O}^{(0)}, \mathcal{R}, \underline{\mathcal{R}}\right)$ such that, for $\delta^{1 / 2} \Delta_{0}$ sufficiently small,

$$
{ }^{(S)} \mathcal{O}_{1,4} \lesssim C
$$

We end this section by deriving a slightly more refined estimate on the second angular derivatives of $\eta$. These estimates are needed in the application to the problem of formation of a trapped surface. We review the system of equations for $\eta$ : written schematically it has the form

$$
\operatorname{curl} \eta=\sigma+\psi \psi, \quad \operatorname{div} \eta=-\mu-\varrho \quad \text { and } \quad \nabla_{4} \mu=\psi(\nabla \psi+\Theta+\Psi+\psi \psi) .
$$

We note the absence of $\operatorname{tr} \underline{\chi}_{0}$ terms in this system. Applying $\mathcal{D}^{*}$ to the Hodge system for $\eta$ and commuting the equation for $\mu$ with $\nabla$, we obtain

$$
\begin{aligned}
\Delta \eta & =\nabla \sigma+\nabla \varrho+\nabla \mu+(\nabla \psi) \psi+K \eta \\
\nabla_{4} \nabla \mu & =(\nabla \psi)(\nabla \psi+\Theta+\Psi+\psi \psi)+\psi\left(\nabla^{2} \psi+\nabla \Theta+\nabla \Psi+(\nabla \psi) \psi\right) .
\end{aligned}
$$

The absence of $\operatorname{tr} \underline{\chi}_{0}$ terms allows us to estimate $\nabla \mu$ in terms of its (trivial) data on $H_{0}$ and an error term of size $\delta^{1 / 2}$. Indeed,

$$
\begin{aligned}
& \|(\nabla \psi)(\nabla \psi+\Theta+\Psi+\psi \psi)\|_{\mathcal{L}_{(\mathrm{sc})}^{2}\left(H_{u}\right)} \\
& \lesssim \delta^{1 / 2}\|\nabla \psi\|_{\mathcal{L}_{(\mathrm{sc})}^{4}(S)}\left(\|\nabla \psi\|_{\mathcal{L}_{(\mathrm{sc})}^{4}(S)}+\|\Theta\|_{\mathcal{L}_{(\mathrm{sc})}^{4}(S)}+\|\Psi\|_{\mathcal{L}_{(\mathrm{sc})}^{4}(S)}\right. \\
& \left.+\delta^{1 / 2}\|\psi\|_{\mathcal{L}_{(\mathrm{sc})}^{\infty}}\|\nabla \psi\|_{\mathcal{L}_{(\mathrm{sc})}^{4}(S)}\right) \\
& +\delta^{1 / 2}\|\psi\|_{\mathcal{L}_{(\mathrm{sc})}^{\infty}}\left(\left\|\nabla^{2} \psi\right\|_{\mathcal{L}_{(\mathrm{sc})}^{2}\left(H_{u}\right)}+\|\nabla \Theta\|_{\mathcal{L}_{(\mathrm{sc})}^{2}\left(H_{u}\right)}+\|\nabla \Psi\|_{\mathcal{L}_{(\mathrm{sc})}^{2}\left(H_{u}\right)}\right. \\
& \left.+\delta^{1 / 2}\|\psi\|_{\mathcal{L}_{(\mathrm{sc})}^{\infty}}\|\nabla \psi\|_{\mathcal{L}_{(\mathrm{sc})}^{2}\left(H_{u}\right)}\right) \\
& \lesssim \delta^{1 / 2} C
\end{aligned}
$$

In the final estimate the only dangerous term is $\|\Psi\|_{\mathcal{L}_{(\mathrm{sc})}^{4}(S)}$, which may be $\delta^{-1 / 4}$ anomalous in the case when $\Psi=\alpha$. It is not difficult to check however that $\Psi=\alpha$ does not appear in this system but, even if it did, the size of the error term would have been $\delta^{1 / 4}$ instead of $\delta^{1 / 2}$. As a result of this estimate and the trivial data for $\nabla \mu$, we obtain

$$
\|\nabla \mu\|_{\mathcal{L}_{(\mathrm{sc})}^{2}(S)} \lesssim \delta^{1 / 2} C .
$$


To estimate $\eta$, we remember that $K=\varrho+\left(\operatorname{tr} \underline{\chi}_{0}\right) \psi_{g}+\psi \psi$. Therefore,

$$
\begin{aligned}
& \|\Delta \eta\|_{\mathcal{L}_{(\mathrm{sc})}^{2}\left(H_{u}\right)} \lesssim\|\nabla \varrho\|_{\mathcal{L}_{(\mathrm{sc})}^{2}\left(H_{u}\right)}+\|\nabla \sigma\|_{\mathcal{L}_{(\mathrm{sc})}^{2}\left(H_{u}\right)}+\|\nabla \mu\|_{\mathcal{L}_{(\mathrm{sc})}^{2}\left(H_{u}\right)} \\
& +\delta^{1 / 2}\|\psi\|_{\mathcal{L}_{(\mathrm{sc})}^{\infty}}\left(\|\nabla \psi\|_{\mathcal{L}_{(\mathrm{sc})}^{2}\left(H_{u}\right)}+\|\varrho\|_{\mathcal{L}_{(\mathrm{sc})}^{2}\left(H_{u}\right)}+\left\|\psi_{g}\right\|_{\mathcal{L}_{(\mathrm{sc})}^{2}\left(H_{u}\right)}\right. \\
& \left.+\delta^{1 / 2}\|\psi\|_{\mathcal{L}_{(\mathrm{sc})}^{\infty}}\|\psi\|_{\mathcal{L}_{(\mathrm{sc})}^{2}\left(H_{u}\right)}\right) \\
& \lesssim\|\nabla \varrho\|_{\mathcal{L}_{(\mathrm{sc})}^{2}\left(H_{u}\right)}+\|\nabla \sigma\|_{\mathcal{L}_{(\mathrm{sc})}^{2}\left(H_{u}\right)}+\delta^{1 / 2} C
\end{aligned}
$$

Using the Böchner identity, we obtain

$$
\begin{aligned}
& \left\|\nabla^{2} \eta\right\|_{\mathcal{L}_{(\mathrm{sc})}^{2}\left(H_{u}\right)} \\
& \quad \lesssim\|\Delta \eta\|_{\mathcal{L}_{(\mathrm{sc})}^{2}\left(H_{u}\right)}+\delta^{1 / 2}\|K\|_{\mathcal{L}_{(\mathrm{sc})}^{2}\left(H_{u}\right)}\|\psi\|_{\mathcal{L}_{(\mathrm{sc})}^{\infty}}+\delta^{1 / 4}\|K\|_{\mathcal{L}_{(\mathrm{sc})}^{2}\left(H_{u}\right)}^{1 / 2}\|\nabla \psi\|_{\mathcal{L}_{(\mathrm{sc})}^{4}(S)} \\
& \quad \lesssim\|\nabla \varrho\|_{\mathcal{L}_{(\mathrm{sc})}^{2}\left(H_{u}\right)}+\|\nabla \sigma\|_{\mathcal{L}_{(\mathrm{sc})}^{2}\left(H_{u}\right)}+\delta^{1 / 4} C .
\end{aligned}
$$

The same estimates also hold along the hypersurface $\underline{H}_{\underline{u}}$.

We summarize this in the following proposition.

Proposition 7.3. The Ricci coefficient $\eta$ satisfies the estimates

$$
\begin{aligned}
& \left\|\nabla^{2} \eta\right\|_{\mathcal{L}_{(\mathrm{sc})}^{2}\left(H_{u}\right)} \lesssim\|\nabla \varrho\|_{\mathcal{L}_{(\mathrm{sc})}^{2}\left(H_{u}\right)}+\|\nabla \sigma\|_{\mathcal{L}_{(\mathrm{sc})}^{2}\left(H_{u}\right)}+\delta^{1 / 4} C, \\
& \left\|\nabla^{2} \eta\right\|_{\mathcal{L}_{(\mathrm{sc})}^{2}\left(\underline{H}_{\underline{u}}\right)} \lesssim\|\nabla \varrho\|_{\mathcal{L}_{(\mathrm{sc})}^{2}\left(\underline{H}_{\underline{u}}\right)}+\|\nabla \sigma\|_{\mathcal{L}_{(\mathrm{sc})}^{2}\left(\underline{H}_{\underline{u}}\right)}+\delta^{1 / 4} C .
\end{aligned}
$$

\section{Remaining first and second derivative estimates}

In the previous sections we have derived estimates on the first and second angular derivatives of the Ricci coefficients. In this section we examine their $\nabla_{3}, \nabla_{4}, \nabla \nabla_{4}$ and $\nabla \nabla_{3}$ derivatives.

\subsection{Direct $\nabla_{3}$ and $\nabla_{4}$ estimates}

These are derived directly from the null structure equations (see $\S 3.1$ ).

Proposition 8.1. There exists a constant $C=C\left(\mathcal{O}^{(0)}, \mathcal{R}, \underline{\mathcal{R}}\right)$ such that, for $\delta^{1 / 2} \Delta_{0}$ sufficiently small and any $S=S_{u, \underline{u}}$,

$$
\begin{aligned}
& \left\|\nabla_{4} \operatorname{tr} \chi\right\|_{\mathcal{L}_{(\mathrm{sc})}^{2}(S)}+\left\|\nabla_{4} \eta\right\|_{\mathcal{L}_{(\mathrm{sc})}^{2}(S)}+\left\|\nabla_{4} \underline{\omega}\right\|_{\mathcal{L}_{(\mathrm{sc})}^{2}(S)}+\left\|\nabla_{4} \operatorname{tr} \underline{\chi}\right\|_{\mathcal{L}_{(\mathrm{sc})}^{2}(S)} \leqslant C, \\
& \left\|\nabla_{3} \widetilde{\operatorname{tr} \underline{\chi}}\right\|_{\mathcal{L}_{(\mathrm{sc})}^{2}(S)}+\left\|\nabla_{3} \underline{\eta}\right\|_{\mathcal{L}_{(\mathrm{sc})}^{2}(S)}+\left\|\nabla_{3} \omega\right\|_{\mathcal{L}_{(\mathrm{sc})}^{2}(S)}+\left\|\nabla_{3} \operatorname{tr} \chi\right\|_{\mathcal{L}_{(\mathrm{sc})}^{2}(S)} \leqslant C, \\
& \left\|\nabla_{4} \hat{\chi}\right\|_{\mathcal{L}_{(\mathrm{sc})}^{2}(S)}+\left\|\nabla_{4} \underline{\hat{\chi}}\right\|_{\mathcal{L}_{(\mathrm{sc})}^{2}(S)}+\left\|\nabla_{3} \widehat{\chi}\right\|_{\mathcal{L}_{(\mathrm{sc})}^{2}(S)}+\left\|\nabla_{3} \underline{\hat{\chi}}\right\|_{\mathcal{L}_{(\mathrm{sc})}^{2}(S)} \leqslant C \delta^{-1 / 2} .
\end{aligned}
$$


Remark. Note the anomalous estimates of the last line. The anomaly of $\nabla_{4} \widehat{\chi}$ is due to the curvature term $\alpha$ in the second equation in (3.3). The anomaly of $\nabla_{3} \underline{\hat{\chi}}$ is due to the term $(\operatorname{tr} \underline{\chi}) \underline{\hat{\chi}}$ in the fourth equation in (3.3). The anomalies for $\nabla_{3} \hat{\chi}$ and $\nabla_{4} \underline{\hat{\chi}}$ are explained by the presence of $(\operatorname{tr} \underline{\chi}) \hat{\chi}$ in both equations of (3.5).

Proof. The claimed estimates follow directly from all the estimates derived so far. We need the full set of $\|\Psi\|_{L^{2}(S)}$ estimates for all null curvature components $\Psi$ which were derived in Propositions 6.9 and 6.11. We also need to make use of the ${ }^{(S)} \mathcal{O}_{0,2}$ estimates of Proposition 5.4. As an example, we prove the estimate for $\nabla_{4} \widehat{\chi}$ in more detail. We start with $\nabla_{4} \widehat{\chi}=-(\operatorname{tr} \chi) \widehat{\chi}-2 \omega \widehat{\chi}-\alpha$, which we write in the form

$$
\nabla_{4} \widehat{\chi}=\psi_{g} \widehat{\chi}+\alpha
$$

As a result,

$$
\begin{aligned}
\left\|\nabla_{4} \widehat{\chi}\right\|_{\mathcal{L}_{(\mathrm{sc})}^{2}(S)} & \lesssim\left\|\psi_{g} \widehat{\chi}\right\|_{\mathcal{L}_{(\mathrm{sc})}^{2}(S)}+\|\alpha\|_{\mathcal{L}_{(\mathrm{sc})}^{2}(S)} \lesssim \delta^{1 / 2}\left\|\psi_{g}\right\|_{\mathcal{L}_{(\mathrm{sc})}^{4}(S)}\|\widehat{\chi}\|_{\mathcal{L}_{(\mathrm{sc})}^{4}}+\|\alpha\|_{\mathcal{L}_{(\mathrm{sc})}^{2}(S)} \\
& \lesssim \delta^{1 / 4(S)} \mathcal{O}_{0,4}^{2}+C \delta^{-1 / 2} \lesssim C \delta^{-1 / 2}
\end{aligned}
$$

as desired. Similarly, we write

$$
\nabla_{3} \widehat{\chi}=\left(\operatorname{tr} \underline{\chi}_{0}\right) \psi_{b}+\psi_{g} \psi_{b}+\nabla \psi
$$

with $\psi_{g}$ and $\Psi_{g}$ non-anomalous and $\psi_{b}$ anomalous. Hence,

$$
\begin{aligned}
\left\|\nabla_{3} \widehat{\chi}\right\|_{\mathcal{L}_{(\mathrm{sc})}^{2}(S)} & \lesssim\left\|\psi_{b}\right\|_{\mathcal{L}_{(\mathrm{sc})}^{2}(S)}+\left\|\psi_{g}\right\|_{\mathcal{L}_{(\mathrm{sc})}^{4}(S)}\|\psi\|_{\mathcal{L}_{(\mathrm{sc})}^{4}(S)}+\|\nabla \psi\|_{\mathcal{L}_{(\mathrm{sc})}^{2}(S)} \\
& \lesssim \delta^{-1 / 2} C+\delta^{1 / 4} C^{2}+C
\end{aligned}
$$

More generally, all of our null structure equations have the form

$$
\begin{aligned}
& \nabla_{4} \psi=\left(\operatorname{tr} \underline{\chi}_{0}\right) \psi+\psi \psi+\nabla \psi+\Psi \\
& \nabla_{3} \psi=\left(\operatorname{tr} \underline{\chi}_{0}\right) \psi+\psi \psi+\nabla \psi+\Psi
\end{aligned}
$$

and one can easily see that the only anomalies occur for $\nabla_{3}$ and $\nabla_{4}$ of $\chi$ and $\underline{\hat{\chi}}$.

\subsection{Estimates for $\nabla_{3} \eta, \nabla_{4} \underline{\eta}, \nabla_{3} \underline{\omega}$ and $\nabla_{4} \omega$}

The above proposition does not address the fate of the $\nabla_{3} \eta, \nabla_{4} \underline{\eta}, \nabla_{3} \underline{\omega}$ and $\nabla_{4} \omega$ derivatives which do not appear in the null structure equations. These can be estimated by commuting the valid transport equations for these quantities with the desired derivative. 
Proposition 8.2. There exists a constant $C=C\left(\mathcal{O}^{(0)}, \mathcal{R}, \underline{\mathcal{R}}\right)$ such that, for $\delta^{1 / 2} \Delta_{0}$ sufficiently small,

$$
\left\|\nabla_{4} \underline{\eta}\right\|_{\mathcal{L}_{(\mathrm{sc})}^{2}(S)}+\left\|\nabla_{4} \omega\right\|_{\mathcal{L}_{(\mathrm{sc})}^{2}(S)}+\left\|\nabla_{3} \eta\right\|_{\mathcal{L}_{(\mathrm{sc})}^{2}(S)}+\left\|\nabla_{3} \underline{\omega}\right\|_{\mathcal{L}_{(\mathrm{sc})}^{2}(S)} \leqslant C
$$

Proof. As all the arguments are similar, we will only derive the estimate for $\nabla_{4} \underline{\eta}$. Commuting the transport equation

$$
\nabla_{3} \underline{\eta}=-\frac{1}{2}(\operatorname{tr} \underline{\chi})(\underline{\eta}-\eta)-\underline{\widehat{\chi}}(\underline{\eta}-\eta)+\underline{\beta}
$$

with $\nabla_{4}$ (according to Lemma 3.1 ), we obtain

$$
\begin{aligned}
\nabla_{3} \nabla_{4} \underline{\eta}=- & \frac{1}{2}\left(\nabla_{4} \operatorname{tr} \underline{\chi}\right)(\underline{\eta}-\eta)-\frac{1}{2}(\operatorname{tr} \underline{\chi}) \nabla_{4}(\underline{\eta}-\eta) \\
& -\left(\nabla_{4} \underline{\chi}\right)(\underline{\eta}-\eta)-\underline{\chi} \nabla_{4}(\underline{\eta}-\eta)+\nabla_{4} \underline{\beta} \\
& -2(\underline{\eta}-\eta) \nabla \underline{\eta}+2 \underline{\omega} \nabla_{4} \underline{\eta}-2 \omega \nabla_{3} \underline{\eta}-2\left(\eta_{a} \underline{\eta}_{b}-\underline{\eta}_{b} \underline{\eta}_{a}-\epsilon_{a b} \sigma\right) \underline{\eta}_{b}
\end{aligned}
$$

which we symbolically write as

$$
\begin{gathered}
\nabla_{3} \nabla_{4} \underline{\eta}=\left(\operatorname{tr} \underline{\chi}_{0}\right)\left(\nabla_{4} \psi_{g}+\nabla_{4} \underline{\eta}+\psi \psi_{g}\right)+\psi\left(\nabla_{4} \psi+\nabla_{4} \underline{\eta}\right) \\
+\psi\left(\nabla \psi+\Psi_{g}+\psi \psi_{g}\right)+\nabla_{4} \underline{\beta} .
\end{gathered}
$$

Remark. In the above expression, $\nabla_{4} \psi$ denotes quantities already controlled by the previous proposition and, among them, $\nabla_{4} \psi_{g}$ denote those which are not anomalous. Also $\Psi_{g}$ is a curvature component different from $\alpha$. Furthermore we can eliminate $\nabla_{4} \beta$ using to the null Bianchi equations

$$
\nabla_{4} \underline{\beta}+(\operatorname{tr} \chi) \underline{\beta}=-\nabla \varrho+{ }^{*} \nabla \sigma+2 \omega \underline{\beta}+2 \underline{\widehat{\chi}} \beta-3\left(\underline{\eta} \varrho-{ }^{*} \underline{\eta} \sigma\right) .
$$

Thus,

$$
\begin{gathered}
\nabla_{3} \nabla_{4} \underline{\eta}=\left(\operatorname{tr} \underline{\chi}_{0}\right)\left(\nabla_{4} \psi_{g}+\nabla_{4} \underline{\eta}+\psi \psi_{g}\right)+\psi\left(\nabla_{4} \psi+\nabla_{4} \underline{\eta}\right) \\
+\psi\left(\nabla \psi+\Psi_{g}+\psi \psi_{g}\right)+\nabla \Psi_{g} .
\end{gathered}
$$

Therefore,

$$
\begin{aligned}
& \left\|\nabla_{3} \nabla_{4} \underline{\eta}\right\|_{\mathcal{L}_{(\mathrm{sc})}^{2}(S)} \\
& \lesssim\left(1+\delta^{1 / 2} \Delta_{0}\right)\left\|\nabla_{4} \underline{\eta}\right\|_{\mathcal{L}_{(\mathrm{sc})}^{2}(S)}+\left\|\nabla_{4} \psi_{g}\right\|_{\mathcal{L}_{(\mathrm{sc})}^{2}(S)}+\delta^{1 / 2} \Delta_{0}\left\|\nabla_{4} \psi\right\|_{\mathcal{L}_{(\mathrm{sc})}^{2}(S)} \\
& +\|\psi\|_{\mathcal{L}_{(\mathrm{sc})}^{\infty}(S)}\left(\left\|\psi_{g}\right\|_{\mathcal{L}_{(\mathrm{sc})}^{2}(S)}+\|\nabla \psi\|_{\mathcal{L}_{(\mathrm{sc})}^{2}(S)}+\left\|\Psi_{g}\right\|_{\mathcal{L}_{(\mathrm{sc})}^{2}(S)}\right)+\left\|\nabla \Psi_{g}\right\|_{\mathcal{L}_{(\mathrm{sc})}^{2}(S)} \\
& \lesssim\left(1+\delta^{1 / 2} \Delta_{0}\right)\left\|\nabla_{4} \underline{\eta}\right\|_{\mathcal{L}_{(\mathrm{sc})}^{2}(S)}+\left\|\nabla \Psi_{g}\right\|_{\mathcal{L}_{(\mathrm{sc})}^{2}(S)}+C .
\end{aligned}
$$


Therefore,

$$
\begin{aligned}
\left\|\nabla_{4} \underline{\eta}\right\|_{\mathcal{L}_{(\mathrm{sc})}^{2}(u, \underline{u}) \lesssim}\left\|\nabla_{4} \underline{\eta}\right\|_{\mathcal{L}_{(\mathrm{sc})}^{2}(0, \underline{u})}+\int_{0}^{u}\left\|\nabla_{3} \nabla_{4} \underline{\eta}\right\|_{\mathcal{L}_{(\mathrm{sc})}^{2}\left(u^{\prime}, \underline{u}\right)} d u^{\prime} \\
\lesssim\left\|\nabla_{4} \underline{\eta}\right\|_{\mathcal{L}_{(\mathrm{sc})}^{2}(0, \underline{u})}+\left(1+\delta^{1 / 2} \Delta_{0}\right) \int_{0}^{u}\left\|\nabla_{4} \underline{\eta}\right\|_{\mathcal{L}_{(\mathrm{sc})}^{2}\left(u^{\prime}, \underline{u}\right)} d u^{\prime} \\
\quad+\int_{0}^{u}\left\|\nabla \Psi_{g}\right\|_{\mathcal{L}_{(\mathrm{sc})}\left(u^{\prime}, \underline{u}\right)} d u^{\prime}+C \\
\lesssim \mathcal{O}^{(0)}+\left(1+\delta^{1 / 2} \Delta_{0}\right) \int_{0}^{u} \| \nabla_{4} \underline{\eta}_{\mathcal{L}_{(\mathrm{sc})}^{2}\left(u^{\prime}, \underline{u}\right)} d u^{\prime}+\underline{\mathcal{R}}_{1}+C
\end{aligned}
$$

Thus, by Grönwall's inequality,

$$
\left\|\nabla_{4} \underline{\eta}\right\|_{\mathcal{L}_{(\mathrm{sc})}^{2}(u, \underline{u})} \lesssim \mathcal{O}^{(0)}+C .
$$

\subsection{Direct angular derivative estimates}

Here we derive angular derivative estimates for all the quantities which appear in Proposition 8.1. We shall first prove the following result.

LEMMA 8.3. If $\delta^{1 / 2} \Delta_{0}$ is small, there exists a constant $C=C\left(\mathcal{O}^{(0)}, \mathcal{R}, \underline{\mathcal{R}}\right)$ such that, for all Ricci coefficients $\psi$,

$$
\left\|\left[\nabla_{4}, \nabla\right] \psi\right\|_{\mathcal{L}_{(\mathrm{sc})}^{2}(S)} \lesssim C \quad \text { and } \quad\left\|\left[\nabla_{3}, \nabla\right] \psi\right\|_{\mathcal{L}_{(\mathrm{sc})}^{2}(S)} \lesssim C .
$$

As a corollary we also have,

$$
\begin{gathered}
\left\|\left[\nabla_{4}, \nabla\right] \psi\right\|_{\mathcal{L}_{(\mathrm{sc})}^{2}(H)}+\left\|\left[\nabla_{4}, \nabla\right] \psi\right\|_{\mathcal{L}_{(\mathrm{sc})}^{2}(\underline{H})} \lesssim C, \\
\left\|\left[\nabla_{3}, \nabla\right] \psi\right\|_{\mathcal{L}_{(\mathrm{sc})}^{2}(H)}+\left\|\left[\nabla_{3}, \nabla\right] \psi\right\|_{\mathcal{L}_{(\mathrm{sc})}^{2}(\underline{H})} \lesssim C .
\end{gathered}
$$

Proof. We write

$$
\begin{aligned}
& {\left[\nabla_{4}, \nabla\right] \psi=\psi \nabla \psi+\beta \psi+\psi_{g} \nabla_{4} \psi+\psi_{g} \psi \psi} \\
& {\left[\nabla_{3}, \nabla\right] \psi=\left(\operatorname{tr} \underline{\chi}_{0}\right) \nabla \psi+\psi \nabla \psi+\underline{\beta} \psi+\psi_{g} \nabla_{3} \psi+\psi_{g} \psi \psi}
\end{aligned}
$$

Hence, in view of the previous estimates ${ }^{(S)} \mathcal{O}_{1,2} \lesssim C$ and $\|\beta\|_{\mathcal{L}_{(\text {sc) }}^{2}(S)} \lesssim C$ and the possibly anomalous estimate $\left\|\nabla_{4} \psi\right\|_{\mathcal{L}_{(\mathrm{sc})}^{2}(S)} \lesssim C \delta^{-1 / 2}$, we derive that

$$
\left\|\left[\nabla_{4}, \nabla\right] \psi\right\|_{\mathcal{L}_{(\mathrm{sc})}^{2}(S)} \lesssim \delta^{1 / 2} \Delta_{0}\left(\|\nabla \psi\|_{\mathcal{L}_{(\mathrm{sc})}^{2}(S)}+\|\beta\|_{\mathcal{L}_{(\mathrm{sc})}^{2}(S)}+\left\|\nabla_{4} \psi\right\|_{\mathcal{L}_{(\mathrm{sc})}^{2}(S)}\right) \lesssim C .
$$

Similarly,

$$
\left\|\left[\nabla_{3}, \nabla\right] \psi\right\|_{\mathcal{L}_{(\mathrm{sc})}^{2}(S)} \lesssim\left(1+\delta^{1 / 2} \Delta_{0}\right)\|\nabla \psi\|_{\mathcal{L}_{(\mathrm{sc})}^{2}(S)}+\delta^{1 / 2} \Delta_{0}\left(\|\underline{\beta}\|_{\mathcal{L}_{(\mathrm{sc})}^{2}(S)}+\left\|\nabla_{3} \psi\right\|_{\mathcal{L}_{(\mathrm{sc})}^{2}(S)}\right) \lesssim C,
$$

from which the estimates of the lemma quickly follow by integration. 
Proposition 8.4. There exists a constant $C=C\left(\mathcal{O}^{(0)}, \mathcal{R}, \underline{\mathcal{R}}\right)$ such that, for $\delta^{1 / 2} \Delta_{0}$ sufficiently small,

$$
\begin{aligned}
& \left\|\nabla \nabla_{4} \chi\right\|_{\mathcal{L}_{\text {(sc) }}^{2}(H)}+\left\|\nabla \nabla_{4} \eta\right\|_{\mathcal{L}_{\text {(sc) }}^{2}(H)}+\left\|\nabla \nabla_{4} \underline{\omega}\right\|_{\mathcal{L}_{\text {(sc) }}^{2}(H)}+\left\|\nabla \nabla_{4} \underline{\chi}\right\|_{\mathcal{L}_{\text {(sc) }}^{2}(H)} \leqslant C, \\
& \left\|\nabla \nabla_{4} \operatorname{tr} \chi\right\|_{\mathcal{L}_{(\mathrm{sc})}^{2}(\underline{H})}+\left\|\nabla \nabla_{4} \eta\right\|_{\mathcal{L}_{\text {(sc) }}^{2}(\underline{H})}+\left\|\nabla \nabla_{4} \underline{\omega}\right\|_{\mathcal{L}_{(\mathrm{sc})}^{2}(\underline{H})}+\left\|\nabla \nabla_{4} \underline{\chi}\right\|_{\mathcal{L}_{\text {(sc) }}^{2}(\underline{H})} \leqslant C, \\
& \left\|\nabla \nabla_{3} \underline{\chi}\right\|_{\mathcal{L}_{\text {(sc) }}^{2}(\underline{H})}+\left\|\nabla \nabla_{3} \underline{\eta}\right\|_{\mathcal{L}_{\text {(sc) }}^{2}(\underline{H})}+\left\|\nabla \nabla_{3} \omega\right\|_{\mathcal{L}_{\text {(sc) }}^{2}(\underline{H})}+\left\|\nabla \nabla_{3} \chi\right\|_{\mathcal{L}_{(\mathrm{sc})}^{2}(\underline{H})} \leqslant C, \\
& \left\|\nabla \nabla_{3} \widetilde{\operatorname{tr} \underline{\chi}}\right\|_{\mathcal{L}_{(\mathrm{sc})}^{2}(H)}+\left\|\nabla \nabla_{3} \underline{\eta}\right\|_{\mathcal{L}_{(\mathrm{sc})}^{2}(H)}+\left\|\nabla \nabla_{3} \omega\right\|_{\mathcal{L}_{(\mathrm{sc})}^{2}(H)}+\left\|\nabla \nabla_{3} \chi\right\|_{\mathcal{L}_{(\mathrm{sc})}^{2}(H)} \leqslant C .
\end{aligned}
$$

Remark 8.5. Note the absence of anomalies. This is analogous to the situation with ${ }^{(S)} \mathcal{O}_{1,2}$ estimates: additional $\nabla$ derivatives eliminate the anomalies due to $\alpha$ and the Ricci coefficients $\hat{\chi}$ and $\underline{\hat{\chi}}$.

Remark 8.6. The quantities $\nabla \nabla_{4} \hat{\chi}$ and $\nabla \nabla_{3} \underline{\hat{\chi}}$ are controlled only along $H$ and $\underline{H}$, respectively. This is due to the absence of the corresponding estimates for $\nabla \alpha$ and $\nabla \underline{\alpha}$ along $\underline{H}$ and $H$, respectively.

Remark 8.7. As a consequence of the lemma above, the same estimates hold true if we reverse the order of differentiation.

Proof. Consider the $\nabla_{4}$ transport equations satisfied by $\psi \in\{\operatorname{tr} \chi, \widehat{\chi}, \eta, \widetilde{\operatorname{tr}} \underline{\underline{\chi}}, \underline{\hat{\chi}}, \underline{\omega}\}$ :

$$
\nabla_{4} \psi=\left(\operatorname{tr} \underline{\chi}_{0}\right) \psi+\psi \psi+\nabla \psi+\Psi_{4}
$$

with curvature components $\Psi_{4} \in\{\alpha, \beta, \varrho, \sigma\}$. Clearly,

$$
\left\|\nabla \nabla_{4} \psi\right\|_{\mathcal{L}_{(\mathrm{sc})}^{2}(H)} \lesssim\left(\left\|\nabla^{2} \psi\right\|_{\mathcal{L}_{(\mathrm{sc})}^{2}(H)}+\left\|\nabla \Psi_{4}\right\|_{\mathcal{L}_{(\mathrm{sc})}^{2}(H)}\right)+\left(1+\delta^{1 / 2}\right)\|\nabla \psi\|_{\mathcal{L}_{(\mathrm{sc})}^{2}(H)} \lesssim C .
$$

Also, along $\underline{H}$,

$$
\left\|\nabla \nabla_{4} \psi\right\|_{\mathcal{L}_{(\mathrm{sc})}^{2}(\underline{H})} \lesssim\left(\left\|\nabla^{2} \psi\right\|_{\mathcal{L}_{(\mathrm{sc})}^{2}(\underline{H})}+\left\|\nabla \Psi_{4}\right\|_{\mathcal{L}_{(\mathrm{sc})}^{2}(\underline{H})}\right)+\left(1+\delta^{1 / 2}\right)\|\nabla \psi\|_{\mathcal{L}_{(\mathrm{sc})}^{2}(\underline{H})} \lesssim C,
$$

provided that $\Psi_{4} \neq \alpha$ (i.e. the original $\psi$ on the left is not $\widehat{\chi}$ ).

On the other hand, the $\nabla_{3}$ transport equations satisfied by $\psi \in\{\operatorname{tr} \chi, \widehat{\chi}, \omega, \widetilde{\operatorname{tr}} \underline{\underline{\chi}}, \underline{\widehat{\chi}}, \underline{\underline{\eta}}\}$ are of the form

$$
\nabla_{3} \psi=\left(\operatorname{tr} \underline{\chi}_{0}\right) \psi+\psi \psi+\nabla \psi+\Psi_{3}
$$

with the curvature components $\Psi_{3} \in\{\varrho, \sigma, \underline{\alpha}, \underline{\beta}\}$. The corresponding estimates follow precisely in the same manner. 


\subsection{Estimates for $\nabla \nabla_{3} \eta, \nabla \nabla_{4} \underline{\eta}, \nabla \nabla_{3} \underline{\omega}$ and $\nabla \nabla_{4} \omega$}

In this subsection we prove the following result.

Proposition 8.8. There exists a constant $C=C\left(\mathcal{O}^{(0)}, \mathcal{R}, \underline{\mathcal{R}}\right)$ such that

$$
\left\|\nabla \nabla_{4} \underline{\eta}\right\|_{\mathcal{L}_{(\mathrm{sc})}^{2}(H)}+\left\|\nabla \nabla_{3} \eta\right\|_{\mathcal{L}_{(\mathrm{sc})}^{2}(H)}+\left\|\nabla \nabla_{4} \underline{\eta}\right\|_{\mathcal{L}_{(\mathrm{sc})}^{2}(\underline{H})}+\left\|\nabla \nabla_{3} \eta\right\|_{\mathcal{L}_{(\mathrm{sc})}^{2}(\underline{H})} \leqslant C .
$$

Remark 8.9. Together with the previous proposition, this proposition allows us to control all angular derivatives of all $\nabla_{3}$ and $\nabla_{4}$ derivatives of all the Ricci coefficients $\widehat{\chi}$, $\omega, \eta, \operatorname{tr} \underline{\chi}, \widetilde{\operatorname{tr}} \underline{\chi}, \underline{\hat{\chi}}, \underline{\omega}$ and $\underline{\eta}$ (in some $\mathcal{L}_{(\mathrm{sc})}^{2}(H)$ or $\mathcal{L}_{(\mathrm{sc})}^{2}(\underline{H})$ or both), except for $\nabla \nabla_{4} \omega$ and $\nabla \nabla_{3} \underline{\omega}$.

Proof. To control $\nabla \nabla_{3} \eta$ and $\nabla \nabla_{4} \underline{\eta}$, we make use of Lemma 6.1. Recall that the reduced mass aspect functions $\mu$ and $\underline{\mu}$ satisfy equations of the form

$$
\begin{aligned}
& \nabla_{4} \mu=\psi\left(\nabla \operatorname{tr} \chi+\nabla \psi+\Psi_{4}\right)+\psi \psi \psi_{g} \\
& \nabla_{3} \underline{\mu}=\left(\operatorname{tr} \underline{\chi}_{0}\right)\left(\nabla \operatorname{tr} \underline{\chi}+\nabla \psi+\Psi_{3}\right)+\psi\left(\nabla \operatorname{tr} \underline{\chi}+\nabla \psi+\Psi_{3}\right)+\left(\operatorname{tr} \underline{\chi}_{0}\right) \psi \psi_{g}+\psi \psi \psi_{g},
\end{aligned}
$$

which are to be coupled with Hodge systems of the form

$$
\mathcal{D}(\eta, \underline{\eta})=(\mu, \underline{\mu})+\varrho+\sigma+\psi \psi .
$$

Here $\Psi_{4} \in\{\alpha \beta, \varrho, \sigma\}$ and $\Psi_{3} \in\{\varrho, \sigma, \underline{\alpha}, \underline{\beta}\}$.

Remark. We note the absence of the Ricci coefficients $\omega$ and $\underline{\omega}$ among the $\psi$ variables in the above equations, in particular among the terms of the form $\nabla \psi$. This fact is very important in view of the lack of estimates for $\nabla \nabla_{4} \omega$ and $\nabla \nabla_{3} \underline{\omega}$. Equally important is the absence of the terms $\left(\operatorname{tr} \underline{\chi}_{0}\right) \psi$ with $\psi \in\{\hat{\chi}, \underline{\hat{\chi}}\}$ in equation (8.1). Such terms would lead to an unmanageable double anomaly.

To estimate $\nabla \nabla_{4} \underline{\eta}$, we need to commute the above equations for $\underline{\eta}$ and $\underline{\mu}$ with $\nabla_{4}$. Making use of Lemma 3.1, we derive that

$$
\begin{aligned}
\nabla_{3} \nabla_{4} \underline{\mu}=\left(\nabla_{4} \operatorname{tr} \underline{\chi}_{0}\right)(\nabla \operatorname{tr} \underline{\chi}+\nabla \psi)+\left(\operatorname{tr} \underline{\chi}_{0}\right)\left(\nabla_{4} \nabla \operatorname{tr} \underline{\chi}+\nabla_{4} \nabla \psi\right)+\psi \nabla \underline{\mu} \\
+\nabla_{4} \psi\left(\nabla \operatorname{tr} \underline{\chi}+\nabla \psi+\Psi_{3}\right)+\psi\left(\nabla_{4} \nabla \operatorname{tr} \underline{\chi}+\nabla_{4} \nabla \psi+\nabla_{4} \Psi_{3}+\nabla_{4} \underline{\eta}\right) \\
+\left(\nabla_{4} \operatorname{tr} \underline{\chi}_{0}\right) \psi \psi_{g}+\left(\operatorname{tr} \underline{\chi}_{0}\right)\left(\nabla_{4} \psi\right) \psi+\left(\nabla_{4} \psi\right) \psi \psi+\underline{\omega} \nabla_{4} \underline{\mu}+\omega \nabla_{3} \underline{\mu}, \\
\mathcal{D} \nabla_{4} \underline{\eta}=\nabla_{4} \underline{\mu}+\nabla_{4}(\varrho, \sigma)+\psi\left(\nabla_{4} \psi+\nabla \underline{\eta}+\Psi_{4}\right) .
\end{aligned}
$$

Proceeding as many times before, we write

$$
\left\|\nabla_{4} \underline{\mu}\right\|_{\mathcal{L}_{\text {(sc) }}^{2}(u, \underline{u})} \lesssim\left\|\nabla_{4} \underline{\mu}\right\|_{\mathcal{L}_{\text {(sc) }}^{2}(0, \underline{u})}+\int_{0}^{u}\left\|\nabla_{3} \nabla_{4} \underline{\mu}\right\|_{\mathcal{L}_{\text {(sc) }}^{2}\left(u^{\prime}, \underline{u}\right)} d u^{\prime}
$$


and $\left(\right.$ with $\left.\underline{H}(u, \underline{u})=H_{u}^{0, \underline{u}}\right)$

$$
\begin{aligned}
\int_{0}^{u}\left\|\nabla_{3} \nabla_{4} \underline{\mu}\right\|_{\mathcal{L}_{\text {(sc) }}^{2}\left(u^{\prime}, \underline{u}\right)} d u^{\prime} \lesssim \int_{0}^{u} & \left\|\nabla_{4} \nabla \psi\right\|_{\mathcal{L}_{(\mathrm{sc})}^{2}\left(u^{\prime}, \underline{u}\right)} d u^{\prime}+\int_{0}^{u}\left\|\underline{\omega} \nabla_{4} \underline{\mu}\right\|_{\mathcal{L}_{(\mathrm{sc})}^{2}\left(u^{\prime}, \underline{u}\right)} d u^{\prime} \\
& +\left\|\left(\nabla_{4} \psi\right) \Psi_{3}\right\|_{\mathcal{L}_{\text {(sc) }}^{2}(\underline{H}(u, \underline{u}))}+\left\|\left(\nabla_{4} \psi\right) \nabla \psi\right\|_{\mathcal{L}_{\text {(sc) }}^{2}(\underline{H}(u, \underline{u}))} \\
& +\left\|\psi \nabla_{4} \psi\right\|_{\mathcal{L}_{\text {(sc) }}^{2}(\underline{H}(u, \underline{u}))}+\left\|\psi \nabla_{4} \Psi_{3}\right\|_{\mathcal{L}_{(\mathrm{sc})}^{2}(\underline{H}(u, \underline{u}))} \\
& +\|\psi \nabla \underline{\mu}\|_{\mathcal{L}_{\text {(sc) }}^{2}(\underline{H}(u, \underline{u}))}+\left\|\omega \nabla_{3} \underline{\mu}\right\|_{\mathcal{L}_{\text {(sc) }}^{2}(\underline{H}(u, \underline{u}))}
\end{aligned}
$$

We have kept on the right only the most problematic terms. We now write

$$
\left\|\left(\nabla_{4} \psi\right) \Psi_{3}\right\|_{\mathcal{L}_{(\mathrm{sc})}^{2}(\underline{H})} \lesssim \delta^{1 / 2}\left\|\nabla_{4} \psi\right\|_{\mathcal{L}_{(\mathrm{sc})}^{4}(\underline{H})}\left\|\Psi_{3}\right\|_{\mathcal{L}_{(\mathrm{sc})}^{4}(\underline{H})}
$$

Using the interpolation estimates of Corollary 4.8,

$$
\begin{aligned}
\left\|\nabla_{4} \psi\right\|_{\mathcal{L}_{(\mathrm{sc})}^{4}(\underline{H})} & \lesssim\left\|\nabla \nabla_{4} \psi\right\|_{\mathcal{L}_{(\mathrm{sc})}^{2}(\underline{H})}^{1 / 2}\left\|\nabla_{4} \psi\right\|_{\mathcal{L}_{(\mathrm{sc})}^{4}(\underline{H})}^{1 / 2}+\delta^{1 / 4}\left\|\nabla_{4} \psi\right\|_{\mathcal{L}_{(\mathrm{sc})}^{2}(\underline{H})}, \\
\left\|\Psi_{3}\right\|_{\mathcal{L}_{(\mathrm{sc})}^{4}(\underline{H})} & \lesssim\left\|\nabla \Psi_{3}\right\|_{\mathcal{L}_{(\mathrm{sc})}^{2}(\underline{H})}^{1 / 2}\left\|\Psi_{3}\right\|_{\mathcal{L}_{(\mathrm{sc})}^{2}(\underline{H})}^{1 / 2}+\delta^{1 / 4}\left\|\Psi_{3}\right\|_{\mathcal{L}_{(\mathrm{sc})}^{2}(\underline{H})} .
\end{aligned}
$$

Taking into account the possible anomaly of $\left\|\nabla_{4} \psi\right\|_{\mathcal{L}_{(\mathrm{sc})}^{2}(S)}$ (recalling also that $\psi$ here differs from $\omega$ and $\underline{\omega} !)$, we deduce that

$$
\left\|\nabla_{4} \psi\right\|_{\mathcal{L}_{(\mathrm{sc})}^{4}(\underline{H})} \lesssim C \delta^{-1 / 4} \quad \text { and } \quad\left\|\Psi_{3}\right\|_{\mathcal{L}_{(\mathrm{sc})}^{4}(\underline{H})} \lesssim C .
$$

Therefore,

$$
\left\|\left(\nabla_{4} \psi\right) \Psi_{3}\right\|_{\mathcal{L}_{(\mathrm{sc})}^{2}(\underline{H})} \lesssim C \delta^{1 / 4}
$$

Similarly, taking into account the estimates for ${ }^{(S)} \mathcal{O}_{1,4}$ of Corollary 7.2 ,

$$
\left\|\left(\nabla_{4} \psi\right) \nabla \psi\right\|_{\mathcal{L}_{(\mathrm{sc})}^{2}(\underline{H})} \lesssim \delta^{1 / 2}\left\|\nabla_{4} \psi\right\|_{\mathcal{L}_{(\mathrm{sc})}^{4}(\underline{H})}\|\nabla \psi\|_{\mathcal{L}_{(\mathrm{sc})}^{4}(\underline{H})} \lesssim C \delta^{1 / 4} .
$$

To estimate $\left\|\psi \nabla_{4} \Psi_{3}\right\|_{\mathcal{L}_{(\mathrm{sc})}^{2}(\underline{H})}$ we write, using the Bianchi equations,

$$
\nabla_{4} \Psi_{3}=\nabla \Psi_{g}+\psi \Psi+\omega \Psi
$$

where $\Psi_{g} \in\{\beta, \varrho, \sigma, \underline{\beta}\}$. Recalling the estimate $\|\Psi\|_{\mathcal{L}_{(\text {sc })}^{2}(\underline{H})} \lesssim C \delta^{-1 / 2}$, encountered before, and $\left\|\nabla \Psi_{g}\right\|_{\mathcal{L}_{(\mathrm{sc})}^{2}(\underline{H})} \lesssim \underline{\mathcal{R}}$, we have

$$
\left\|\psi \nabla_{4} \Psi_{3}\right\|_{\mathcal{L}_{(\mathrm{sc})}^{2}(\underline{H})} \lesssim \delta^{1 / 2}\|\psi\|_{\mathcal{L}_{\text {(sc) }}^{\infty}(\underline{H})}\left(\left\|\nabla \Psi_{g}\right\|_{\mathcal{L}_{(\mathrm{sc})}^{2}(\underline{H})}+\|\Psi\|_{\mathcal{L}_{(\mathrm{sc})}^{2}(\underline{H})}\right) \lesssim C .
$$


The term $\left\|\left(\nabla_{4} \psi\right) \psi\right\|_{\mathcal{L}_{\text {(sc) }}^{2}(\underline{H})}$ may contain a double anomaly. We estimate it as

$$
\left\|\left(\nabla_{4} \psi\right) \psi\right\|_{\mathcal{L}_{(\mathrm{sc})}^{2}(\underline{H})} \lesssim \delta^{1 / 2}\|\psi\|_{\mathcal{L}_{\text {(sc) }}^{\infty}}\left\|\nabla_{4} \psi\right\|_{\mathcal{L}_{(\mathrm{sc})}^{2}(\underline{H})} \lesssim C
$$

All other terms in $\mathcal{L}_{(\mathrm{sc})}^{2}(\underline{H})$ can be estimated in the same manner to derive

$$
\begin{gathered}
\left\|\nabla_{4} \underline{\mu}\right\|_{\mathcal{L}_{\text {(sc) }}^{2}(u, \underline{u})} \lesssim\left\|\nabla_{4} \underline{\mu}\right\|_{\mathcal{L}_{(\mathrm{sc})}^{2}(0, \underline{u})}+\int_{0}^{u}\left\|\nabla_{4} \underline{\mu}\right\|_{\mathcal{L}_{(\mathrm{sc})}^{2}\left(u^{\prime}, \underline{u}\right)} d u^{\prime} \\
+\int_{0}^{u}\left\|\nabla_{4} \nabla \psi\right\|_{\mathcal{L}_{\text {(sc) }}^{2}\left(u^{\prime}, \underline{u}\right)} d u^{\prime}+C
\end{gathered}
$$

or, by Grönwall's inequality,

$$
\left\|\nabla_{4} \underline{\mu}\right\|_{\mathcal{L}_{\text {(sc) }}^{2}(u, \underline{u})} \lesssim\left\|\nabla_{4} \underline{\mu}\right\|_{\mathcal{L}_{(\mathrm{sc})}^{2}(0, \underline{u})}+\int_{0}^{u}\left\|\nabla_{4} \nabla \psi\right\|_{\mathcal{L}_{(\mathrm{sc})}^{2}\left(u^{\prime}, \underline{u}\right)} d u^{\prime}+C .
$$

Now,

$$
\int_{0}^{u}\left\|\nabla_{4} \nabla \psi\right\|_{\mathcal{L}_{\text {(sc) }}^{2}\left(u^{\prime}, \underline{u}\right)} d u^{\prime} \lesssim \int_{0}^{u}\left\|\nabla_{4} \nabla \underline{\eta}\right\|_{\mathcal{L}_{\text {(sc) }}^{2}\left(u^{\prime}, \underline{u}\right)} d u^{\prime}+\left\|\nabla_{4} \nabla \psi_{g}\right\|_{\mathcal{L}_{\text {(sc) }}^{2}(\underline{H}(u, \underline{u}))},
$$

where $\psi_{g} \in\{\operatorname{tr} \chi, \widehat{\chi}, \eta, \widetilde{\operatorname{tr} \underline{\chi}}, \widehat{\chi}\}$. Thus, in view of the estimates of Lemma 8.3 and Proposition 8.4,

$$
\int_{0}^{u}\left\|\nabla_{4} \nabla \psi\right\|_{\mathcal{L}_{\text {(sc) }}^{2}\left(u^{\prime}, \underline{u}\right)} d u^{\prime} \lesssim \int_{0}^{u}\left\|\nabla \nabla_{4} \underline{\eta}\right\|_{\mathcal{L}_{\text {(sc) }}^{2}\left(u^{\prime}, \underline{u}\right)} d u^{\prime}+C,
$$

and therefore

$$
\left\|\nabla_{4} \underline{\mu}\right\|_{\mathcal{L}_{\text {(sc) }}^{2}(u, \underline{u})} \lesssim\left\|\nabla_{4} \underline{\mu}\right\|_{\mathcal{L}_{(\mathrm{sc})}^{2}(0, \underline{u})}+\int_{0}^{u}\left\|\nabla \nabla_{4} \underline{\eta}\right\|_{\mathcal{L}_{(\mathrm{sc})}^{2}\left(u^{\prime}, \underline{u}\right)} d u^{\prime}+C .
$$

Using the elliptic estimates of Proposition 4.11 applied to the Hodge system for $\nabla_{4} \psi$, we derive that

$$
\begin{aligned}
\left\|\nabla \nabla_{4} \underline{\eta}\right\|_{\mathcal{L}_{\text {(sc) }}^{2}(S) \lesssim \|} & \nabla_{4} \underline{\mu}\left\|_{\mathcal{L}_{\text {(sc) }}^{2}(S)}+\right\| \nabla_{4}(\varrho, \sigma) \|_{\mathcal{L}_{\text {(sc) }}^{2}(S)} \\
& +\delta^{1 / 2} \Delta_{0}\left(\left\|\nabla_{4} \psi\right\|_{\mathcal{L}_{\text {(sc) }}^{2}(S)}+\|\nabla \psi\|_{\mathcal{L}_{\text {(sc) }}^{2}(S)}+\left\|\Psi_{4}\right\|_{\mathcal{L}_{\text {(sc) }}^{2}(S)}\right) \\
& +\delta^{1 / 2}\|K\|_{\mathcal{L}_{\text {(sc) }}^{2}(S)}\|\psi\|_{\mathcal{L}_{\text {(sc) }}^{2}(S)}
\end{aligned}
$$

Now,

$$
\nabla_{4}(\varrho, \sigma)=\nabla \beta+\psi \Psi_{4}+\omega \Psi_{4},
$$

with $\Psi_{4} \in\{\alpha, \beta, \varrho, \sigma\}$. Moreover,

$$
\left\|\nabla_{4}(\varrho, \sigma)\right\|_{\mathcal{L}_{(\mathrm{sc})}^{2}(S)} \lesssim\|\nabla \beta\|_{\mathcal{L}_{\text {(sc) }}^{2}(S)}+\delta^{1 / 2} \Delta_{0}\left\|\Psi_{4}\right\|_{\mathcal{L}_{(\mathrm{sc})}^{2}(S)} .
$$


In the particular case when $\Psi_{4}=\alpha$ (recall that the $\alpha$ component is not allowed in the definition of the curvature norms $\underline{\mathcal{R}}$ ), we recall (see Proposition 6.11) the estimate

$$
\|\alpha\|_{\mathcal{L}_{(\mathrm{sc})}^{2}(S)} \lesssim \delta^{-1 / 2} C .
$$

Therefore, in all cases,

$$
\|\Psi\|_{\mathcal{L}_{(\mathrm{sc})}^{2}(S)} \lesssim C \delta^{-1 / 2}
$$

and consequently

$$
\left\|\nabla_{4}(\varrho, \sigma)\right\|_{\mathcal{L}_{(\mathrm{sc})}^{2}(S)} \lesssim\|\nabla \beta\|_{\mathcal{L}_{(\mathrm{sc})}^{2}(S)}+C,
$$

with $C=C\left(\mathcal{O}^{(0)}, \mathcal{R}, \underline{\mathcal{R}}\right)$. Therefore,

$$
\left\|\nabla \nabla_{4} \underline{\eta}\right\|_{\mathcal{L}_{(\mathrm{sc})}^{2}(S)} \lesssim\left\|\nabla_{4} \underline{\mu}\right\|_{\mathcal{L}_{(\mathrm{sc})}^{2}(S)}+\|\nabla \beta\|_{\mathcal{L}_{(\mathrm{sc})}^{2}(S)}+C .
$$

Integrating,

$$
\begin{aligned}
\int_{0}^{u}\left\|\nabla \nabla_{4} \underline{\eta}\right\|_{\mathcal{L}_{(\mathrm{sc})}^{2}\left(u^{\prime}, \underline{u}\right)} d u^{\prime} & \lesssim \int_{0}^{u}\left\|\nabla_{4} \underline{\mu}\right\|_{\mathcal{L}_{(\mathrm{sc})}^{2}\left(u^{\prime}, \underline{u}\right)} d u^{\prime}+\int_{0}^{u}\|\nabla \beta\|_{\mathcal{L}_{(\mathrm{sc})}^{2}\left(u^{\prime}, \underline{u}\right)} d u^{\prime}+C \\
& \lesssim \int_{0}^{u}\left\|\nabla_{4} \underline{\mu}\right\|_{\mathcal{L}_{(\mathrm{sc})}^{2}\left(u^{\prime}, \underline{u}\right)} d u^{\prime}+\underline{\mathcal{R}}+C,
\end{aligned}
$$

i.e.,

$$
\int_{0}^{u}\left\|\nabla \nabla_{4} \underline{\eta}\right\|_{\mathcal{L}_{\text {(sc) }}^{2}\left(u^{\prime}, \underline{u}\right)} d u^{\prime} \lesssim \int_{0}^{u}\left\|\nabla_{4} \underline{\mu}\right\|_{\mathcal{L}_{\text {(sc) }}^{2}\left(u^{\prime}, \underline{u}\right)} d u^{\prime}+C .
$$

Combining with (8.3) and applying Grönwall's inequality again, we deduce that

$$
\left\|\nabla_{4} \underline{\mu}\right\|_{\mathcal{L}_{(\mathrm{sc})}^{2}(u, \underline{u})} \lesssim\left\|\nabla_{4} \underline{\mu}\right\|_{\mathcal{L}_{(\mathrm{sc})}^{2}(0, \underline{u})}+C .
$$

It is easy to check, on the initial hypersurface $H_{0}$, that

$$
\left\|\nabla_{4} \underline{\mu}\right\|_{\mathcal{L}_{(\mathrm{sc})}^{2}(0, \underline{u})} \lesssim \mathcal{O}^{(0)} .
$$

On the other hand, returning to (8.4), we deduce that

$$
\left\|\nabla \nabla_{4}{ }_{\underline{\eta}}\right\|_{\mathcal{L}_{(\mathrm{sc})}^{2}(S)} \lesssim C+\|\nabla \beta\|_{\mathcal{L}_{(\mathrm{sc})}^{2}(S)} .
$$

Hence,

$$
\left\|\nabla \nabla_{4} \underline{\eta}\right\|_{\mathcal{L}_{(\mathrm{sc})}^{2}(H)}+\left\|\nabla \nabla_{4} \underline{\eta}\right\|_{\mathcal{L}_{\text {(sc) }}^{2}(\underline{H})} \lesssim C
$$

as desired.

The remaining estimate

$$
\left\|\nabla \nabla_{3} \eta\right\|_{\mathcal{L}_{(\mathrm{sc})}^{2}(H)}+\left\|\nabla \nabla_{3} \eta\right\|_{\mathcal{L}_{(\mathrm{sc})}^{2}(\underline{H})} \lesssim C
$$

is proved in exactly the same manner. 


\section{9. $\mathcal{O}_{\infty}$ estimates and proof of Theorem A}

In this section we combine the estimates obtained so far to derive $L^{\infty}$ estimates for all our Ricci coefficients and thus verify the bootstrap assumption (2.13). This will also allow us to conclude the proof of Theorem A. To achieve this, we combine the ${ }^{(S)} \mathcal{O}_{0,4}$, $\mathcal{O}_{1,2},{ }^{(H)} \mathcal{O},{ }^{(H)} \mathcal{O}$ and the remaining second derivative estimates with the interpolation results of Proposition 4.10. We will only require results before and culminating with Proposition 8.4. In particular, we do not need the estimates of Proposition 8.8.

For the Ricci coefficients $\psi \in\{\operatorname{tr} \chi, \widehat{\chi}, \omega, \eta\}$ we make use of the interpolation estimate of Proposition 4.10 together with ${ }^{(S)} \mathcal{O}_{1,2}+{ }^{(H)} \mathcal{O} \lesssim C$ and $\left\|\nabla_{4} \nabla \psi\right\|_{\mathcal{L}_{(\text {sc })}^{2}(H)} \lesssim C$ of Proposition 8.4 , to derive that

$$
\begin{aligned}
\|\nabla \psi\|_{\mathcal{L}_{(\mathrm{sc})}^{4}(S)} \lesssim( & \left.\delta^{1 / 2}\|\nabla \psi\|_{\mathcal{L}_{(\mathrm{sc})}^{2}(H)}+\left\|\nabla^{2} \psi\right\|_{\mathcal{L}_{(\mathrm{sc})}^{2}(H)}\right)^{1 / 2} \\
& \times\left(\delta^{1 / 2}\|\nabla \psi\|_{\mathcal{L}_{(\mathrm{sc})}^{2}(H)}+\left\|\nabla_{4} \nabla \psi\right\|_{\mathcal{L}_{(\mathrm{sc})}^{2}(H)}\right)^{1 / 2} \lesssim C .
\end{aligned}
$$

Similarly, for $\psi \in\{\widetilde{\operatorname{tr} \underline{\chi}}, \underline{\widehat{\chi}}, \underline{\omega}, \underline{\eta}\}$, using the estimates ${ }^{(S)} \mathcal{O}_{1,2}+{ }^{(\underline{H})} \mathcal{O} \lesssim C$ and estimate

$$
\left\|\nabla_{3} \nabla \psi\right\|_{\mathcal{L}_{(\mathrm{sc})}^{2}(H)} \lesssim C
$$

of Proposition 8.4, we get

$$
\begin{aligned}
\|\nabla \psi\|_{\mathcal{L}_{(\mathrm{sc})}^{4}(S)} \lesssim\left(\delta^{1 / 2}\|\nabla \psi\|_{\mathcal{L}_{(\mathrm{sc})}^{2}(\underline{H})}+\left\|\nabla^{2} \psi\right\|_{\mathcal{L}_{(\mathrm{sc})}^{2}(\underline{H})}\right)^{1 / 2} & \\
& \times\left(\delta^{1 / 2}\|\nabla \psi\|_{\mathcal{L}_{(\mathrm{sc})}^{2}(\underline{H})}+\left\|\nabla_{3} \nabla \psi\right\|_{\mathcal{L}_{(\mathrm{sc})}^{2}(\underline{H})}\right)^{1 / 2} \lesssim C .
\end{aligned}
$$

Next, for the non-anomalous coefficients $\psi \in\{\operatorname{tr} \chi, \omega, \eta, \widetilde{\operatorname{tr}} \underline{\chi}, \underline{\omega}, \underline{\eta}\}$, we use the interpolation inequality

$$
\|\psi\|_{\mathcal{L}_{(\mathrm{sc})}^{\infty}(S)} \lesssim\|\nabla \psi\|_{\mathcal{L}_{(\mathrm{sc})}^{4}(S)}^{1 / 2}\|\psi\|_{\mathcal{L}_{(\mathrm{sc})}^{4}(S)}^{1 / 2}+\delta^{1 / 4}\|\psi\|_{\mathcal{L}_{(\mathrm{sc})}^{4}(S)}
$$

which leads to the desired estimate

$$
\|\psi\|_{\mathcal{L}_{(\mathrm{sc})}^{\infty}(S)} \lesssim C .
$$

In the anomalous case when $\psi \in\{\hat{\chi}, \underline{\hat{\chi}}\}$, we use the interpolation inequality (4.22)

$$
\|\psi\|_{\mathcal{L}_{(\mathrm{sc})}^{\infty}(S)} \lesssim \sup _{\delta S}\left(\|\nabla \psi\|_{\mathcal{L}_{(\mathrm{sc})}^{4}(S)}+\|\psi\|_{\mathcal{L}_{(\mathrm{sc})}^{4}\left({ }^{\prime} S\right)}\right)
$$

which gives

$$
\|\psi\|_{\mathcal{L}_{(\mathrm{sc})}^{\infty}(S)} \lesssim C .
$$

as desired. We deduce the following result.

Proposition 9.1. There exists a constant $C=C\left(\mathcal{O}^{(0))}, \mathcal{R}, \underline{\mathcal{R}}\right)$ such that, for $\delta^{1 / 2} \Delta_{0}$ sufficiently small, we have

$$
{ }^{(S)} \mathcal{O}_{0, \infty} \lesssim C
$$

In particular, choosing $\Delta_{0} \approx C$ and $\delta>0$ sufficiently small, depending only on $C$, we dispense of the bootstrap assumption and derive the conclusion of Theorem A. 


\section{0. $\mathcal{L}_{(\mathrm{sc})}^{4}(S)$ estimates for curvature and the first derivatives of the Ricci coefficients}

In this section we establish $\mathcal{L}_{(\mathrm{sc})}^{4}(S)$ estimates for all first derivatives of the Ricci coefficients $\psi$. In the previous section we have already established such estimates for $\nabla \psi$. The Ricci coefficients satisfy the structure equations

$$
\nabla_{4} \psi=\left(\operatorname{tr} \underline{\chi}_{0}\right) \psi+\psi \psi+\nabla \psi+\Psi \quad \text { and } \quad \nabla_{3} \psi=\left(\operatorname{tr} \underline{\chi}_{0}\right) \psi+\psi \psi+\nabla \psi+\Psi
$$

We note that the double anomalous terms $\left(\operatorname{tr} \underline{\chi}_{0}\right) \hat{\chi}$ and $\left(\operatorname{tr} \underline{\chi}_{0}\right) \underline{\hat{\chi}}$ appear only in the $\nabla_{4} \underline{\hat{\chi}}$, $\nabla_{3} \hat{\chi}$ and $\nabla_{3} \underline{\hat{\chi}}$ equations. Similarly, the anomalous $\alpha$ curvature component only appears in the $\nabla_{4} \widehat{\chi}$ equation.

For the remaining equations, we estimate

$$
\begin{aligned}
\left\|\nabla_{4} \psi\right\|_{\mathcal{L}_{(\mathrm{sc})}^{4}(S)} & \lesssim\|\psi\|_{\mathcal{L}_{(\mathrm{sc})}^{4}(S)}+\delta^{1 / 2}\|\psi\|_{\mathcal{L}_{(\mathrm{sc})}^{\infty}(S)}\|\psi\|_{\mathcal{L}_{(\mathrm{sc})}^{4}(S)}+\|\nabla \psi\|_{\mathcal{L}_{(\mathrm{sc})}^{4}(S)}+\|\Psi\|_{\mathcal{L}_{(\mathrm{sc})}^{4}(S)} \\
& \lesssim \mathcal{O}_{0,4}+\delta^{1 / 4} \mathcal{O}_{0, \infty} \mathcal{O}_{0,4}+\mathcal{O}_{1,4}+\|\Psi\|_{\mathcal{L}_{(\mathrm{sc})}^{4}(S)}
\end{aligned}
$$

where the $\delta^{1 / 4}$ takes into account a potential anomaly of the $\|\psi\|_{\mathcal{L}_{(\mathrm{sc})}^{4}(S)}$ term. To estimate $\|\Psi\|_{\mathcal{L}_{(\mathrm{sc})}^{4}(S)}$ we use the interpolation estimates

$$
\begin{aligned}
\|\Psi\|_{\mathcal{L}_{(\mathrm{sc})}^{4}(S)} & \lesssim\left(\delta^{1 / 2}\|\Psi\|_{\mathcal{L}_{(\mathrm{sc})}^{2}(H)}+\|\nabla \Psi\|_{\mathcal{L}_{(\mathrm{sc})}^{2}(H)}\right)^{1 / 2}\left(\delta^{1 / 2}\|\Psi\|_{\mathcal{L}_{(\mathrm{sc})}^{2}(H)}+\left\|\nabla_{4} \Psi\right\|_{\mathcal{L}_{(\mathrm{sc})}^{2}(H)}\right)^{1 / 2} \\
\|\Psi\|_{\mathcal{L}_{(\mathrm{sc})}^{4}(S)} & \lesssim\left(\delta^{1 / 2}\|\Psi\|_{\mathcal{L}_{(\mathrm{sc})}^{2}(\underline{H})}+\|\nabla \Psi\|_{\mathcal{L}_{(\mathrm{sc})}^{2}(\underline{H})}\right)^{1 / 2}\left(\delta^{1 / 2}\|\Psi\|_{\mathcal{L}_{(\mathrm{sc})}^{2}(\underline{H})}+\left\|\nabla_{3} \Psi\right\|_{\mathcal{L}_{(\mathrm{sc})}^{2}(\underline{H})}\right)^{1 / 2}
\end{aligned}
$$

Each of the null curvature components $\Psi$ satisfies either the $\nabla_{4}$ or $\nabla_{3}$ equation. These equations can be written schematically in the form

$$
\begin{aligned}
& \nabla_{4} \Psi^{(s)}=\nabla \Psi^{(s+1 / 2)}+\sum_{s_{1}+s_{2}=s+1} \psi^{\left(s_{1}\right)} \Psi^{\left(s_{2}\right)}, \\
& \nabla_{3} \Psi^{(s)}=\nabla \Psi^{(s-1 / 2)}+\left(\operatorname{tr} \underline{\chi}_{0}\right) \Psi^{s}+\sum_{s_{1}+s_{2}=s} \psi^{\left(s_{1}\right)} \Psi^{\left(s_{2}\right)} .
\end{aligned}
$$

Let us consider the $\nabla_{3}$ equation, since the presence of the $\operatorname{tr} \underline{\chi}_{0}$ makes it more difficult to handle. We estimate

$$
\begin{aligned}
\left\|\nabla_{3} \Psi^{(s)}\right\|_{\mathcal{L}_{(\mathrm{sc})}^{2}(\underline{H}) \lesssim} & \left\|\nabla \Psi^{(s-1 / 2)}\right\|_{\mathcal{L}_{(\mathrm{sc})}^{2}(\underline{H})}+\left\|\Psi^{s}\right\|_{\mathcal{L}_{(\mathrm{sc})}^{2}(\underline{H})} \\
& +\delta^{1 / 2} \sum_{s_{1}+s_{2}=s}\left\|\psi^{\left(s_{1}\right)}\right\|_{\mathcal{L}_{(\mathrm{sc})}^{\infty}}\left\|\Psi^{\left(s_{2}\right)}\right\|_{\mathcal{L}_{(\mathrm{sc})}^{2}(\underline{H})}
\end{aligned}
$$

Note that the terms $\left\|\Psi^{s}\right\|_{\mathcal{L}_{(\mathrm{sc})}^{2}(\underline{H})}$ and $\left\|\Psi^{s_{2}}\right\|_{\mathcal{L}_{(\mathrm{sc})}^{2}(\underline{H})}$ are anomalous only for $s=s_{2}=2$, that is in the case of the estimate for $\alpha$. We summarize these estimates in the following result. 
Lemma 10.1. For a constant $C=C(\mathcal{I}, \mathcal{O}, \mathcal{R}, \underline{\mathcal{R}})$ and $\Psi \in\{\beta, \varrho, \sigma, \underline{\alpha}, \underline{\beta}\}$,

$$
\delta^{1 / 4}\|\alpha\|_{\mathcal{L}_{(\mathrm{sc})}^{4}(S)}+\|\Psi\|_{\mathcal{L}_{(\mathrm{sc})}^{4}(S)} \leqslant C .
$$

Combining this result with $\nabla_{4} \psi$ and $\nabla_{3} \psi$ equations, as described above, gives us the estimates

$$
\left\|\nabla_{4} \psi\right\|_{\mathcal{L}_{(\mathrm{sc})}^{4}(S)}+\left\|\nabla_{3} \psi\right\|_{\mathcal{L}_{(\mathrm{sc})}^{4}(S)} \leqslant C
$$

for those derivatives, with the exception of $\psi \in\{\hat{\chi}, \hat{\chi}\}$. On the other hand, the anomalies present in their respective equations lead to the anomalous estimates

$$
\left\|\nabla_{4} \hat{\chi}\right\|_{\mathcal{L}_{(\mathrm{sc})}^{4}(S)}+\left\|\nabla_{3} \hat{\chi}\right\|_{\mathcal{L}_{(\mathrm{sc})}^{4}(S)}+\left\|\nabla_{4} \underline{\hat{\chi}}_{\mathcal{L}_{(\mathrm{sc})}^{4}(S)}+\right\| \nabla_{3} \underline{\hat{\chi}} \|_{\mathcal{L}_{(\mathrm{sc})}^{4}(S)} \leqslant C \delta^{-1 / 4} .
$$

It remains to estimate $\nabla_{3} \eta, \nabla_{4} \underline{\eta}, \nabla_{3} \underline{\omega}$ and $\nabla_{4} \omega$ which do not satisfy direct equations. We argue as in $\S 8.2$ and $\S 8.4$. Using the interpolation estimates stated in the beginning of this section and the bounds

$$
\begin{gathered}
\left\|\nabla \nabla_{3} \eta\right\|_{\mathcal{L}_{\text {(sc) }}^{2}(H)}+\left\|\nabla_{4} \nabla_{3} \eta\right\|_{\mathcal{L}_{\text {(sc) }}^{2}(H)} \leqslant C, \\
\left\|\nabla \nabla_{4} \underline{\eta}\right\|_{\mathcal{L}_{\text {(sc) }}^{2}(H)}+\left\|\nabla_{3} \nabla_{4} \underline{\eta}\right\|_{\mathcal{L}_{\text {(sc) }}^{2}(H)} \leqslant C
\end{gathered}
$$

of $\S 8.2$ and $\S 8.4$, we obtain the desired $\mathcal{L}_{\text {(sc) }}^{4}(S)$ estimates for $\nabla_{3} \eta$ and $\nabla_{4} \underline{\eta}$. However, we cannot obtain the corresponding estimates for $\nabla_{4} \omega$ and $\nabla_{3} \underline{\omega}$. We summarize the second main result of this section.

LEMMA 10.2 .

$$
\begin{aligned}
& \|\nabla \psi\|_{\mathcal{L}_{(\mathrm{sc})}^{4}(S)}+\left\|\nabla_{3,4} \eta\right\|_{\mathcal{L}_{(\mathrm{sc})}^{4}(S)}+\left\|\nabla_{3,4} \underline{\eta}\right\|_{\mathcal{L}_{(\mathrm{sc})}^{4}(S)}+\left\|\nabla_{4} \underline{\omega}\right\|_{\mathcal{L}_{(\mathrm{sc})}^{4}(S)}+\left\|\nabla_{3} \omega\right\|_{\mathcal{L}_{(\mathrm{sc})}^{4}(S)} \leqslant C, \\
& \left\|\nabla_{4} \widehat{\chi}\right\|_{\mathcal{L}_{(\mathrm{sc})}^{4}(S)}+\left\|\nabla_{3} \hat{\chi}\right\|_{\mathcal{L}_{(\mathrm{sc})}^{4}(S)}+\left\|\nabla_{4} \underline{\hat{\chi}}\right\|_{\mathcal{L}_{(\mathrm{sc})}^{4}(S)}+\left\|\nabla_{3} \underline{\hat{\chi}}\right\|_{\mathcal{L}_{(\mathrm{sc})}^{4}(S)} \leqslant C \delta^{-1 / 4} .
\end{aligned}
$$

\section{Renormalized estimates}

\subsection{Trace theorems}

The results of this section rely on sharp trace theorems which we discuss below. We introduce the following new norms for an $S$-tangent tensor $\phi$ with scale $\operatorname{sc}(\phi)$ along $H=H_{u}^{(0, \underline{u})}$, relative to the transported coordinates $(\underline{u}, \theta)$ of Proposition 4.4,

$$
\|\phi\|_{\operatorname{Tr}_{(\mathrm{sc})}(H)}=\delta^{-\operatorname{sc}(\phi)-1 / 2}\left(\sup _{\theta \in S(u, 0)} \int_{0}^{\underline{u}}\left|\phi\left(u, \underline{u}^{\prime}, \theta\right)\right|^{2} d \underline{u}^{\prime}\right)^{1 / 2} .
$$

Also, along $\underline{H}=\underline{H}_{\underline{u}}^{(0, u)}$ relative to the transported coordinates $(u, \underline{\theta})$ of Proposition 4.4,

$$
\|\phi\|_{\operatorname{Tr}_{(\mathrm{sc})}(\underline{H})}=\delta^{-\operatorname{sc}(\phi)}\left(\sup _{\underline{\theta} \in S(\underline{u}, 0)} \int_{0}^{u}\left|\phi\left(u^{\prime}, \underline{u}, \underline{\theta}\right)\right|^{2} d u^{\prime}\right)^{1 / 2} .
$$


Proposition 11.1. For any horizontal tensor $\phi$ along $H=H_{u}^{(0, \underline{u})}$,

$$
\begin{aligned}
& \left\|\nabla_{4} \phi\right\|_{\operatorname{Tr}_{(\mathrm{sc})}(H)} \lesssim\left(\left\|\nabla_{4}^{2} \phi\right\|_{\mathcal{L}_{\text {(sc) }}^{2}(H)}+\|\phi\|_{\mathcal{L}_{\text {(sc) }}^{2}(H)}+\delta^{1 / 2} C\left(\|\phi\|_{\mathcal{L}_{\text {(sc) }}^{\infty}}+\left\|\nabla_{4} \phi\right\|_{\mathcal{L}_{(\mathrm{sc})}^{4}(S)}\right)\right)^{1 / 2} \\
& \times\left(\left\|\nabla^{2} \phi\right\|_{\mathcal{L}_{(\mathrm{sc})}^{2}(H)}+\delta^{1 / 2} C\left(\|\phi\|_{\mathcal{L}_{(\mathrm{sc})}^{\infty}}+\|\nabla \phi\|_{\mathcal{L}_{(\mathrm{sc})}^{4}(S)}\right)\right)^{1 / 2} \\
& +\left\|\nabla_{4} \nabla \phi\right\|_{\mathcal{L}_{(\mathrm{sc})}^{2}(H)}+\|\phi\|_{\mathcal{L}_{(\mathrm{sc})}^{\infty}}+\|\nabla \phi\|_{\mathcal{L}_{(\mathrm{sc})}^{2}(H)},
\end{aligned}
$$

where $C$ is a constant which depends on $\mathcal{O}^{(0)}, \mathcal{R}$ and $\underline{\mathcal{R}}$.

Also, for any horizontal tensor $\phi$ along $\underline{H}=H_{\underline{u}}^{(u, 0)}$, and a similar constant $C$,

$$
\begin{aligned}
& \left\|\nabla_{3} \phi\right\|_{\operatorname{Tr}_{(\text {(sc })}(\underline{H})} \lesssim\left(\left\|\nabla_{3}^{2} \phi\right\|_{\mathcal{L}_{\text {(sc) }}^{2}(\underline{H})}+\|\phi\|_{\mathcal{L}_{\text {(sc) }}^{2}(\underline{H})}+\delta^{1 / 2} C\left(\|\phi\|_{\mathcal{L}_{\text {(sc) }}^{\infty}}+\left\|\nabla_{3} \phi\right\|_{\mathcal{L}_{(\mathrm{sc})}^{4}(S)}\right)\right)^{1 / 2} \\
& \times\left(\left\|\nabla^{2} \phi\right\|_{\mathcal{L}_{(\mathrm{sc})}^{2}(H)}+\delta^{1 / 2} C\left(\|\phi\|_{\mathcal{L}_{(\mathrm{sc})}^{\infty}}+\|\nabla \phi\|_{\mathcal{L}_{(\mathrm{sc})}^{4}(S)}\right)\right)^{1 / 2} \\
& +\left\|\nabla_{3} \nabla \phi\right\|_{\mathcal{L}_{\text {(sc) }}^{2}(\underline{H})}+\|\phi\|_{\mathcal{L}_{\text {(sc) }}^{\infty}}+\|\nabla \phi\|_{\mathcal{L}_{\text {(sc) }}^{2}(\underline{H})} .
\end{aligned}
$$

The proof relies on the classical (euclidean) trace inequality formulated in $(u, \theta)$ or $(\underline{u}, \theta)$ coordinates.

LEMma 11.2. For any scalar function $\phi$ along $H=H_{u}^{(0, \underline{u})}$, supported in a coordinate chart, we have

$$
\begin{gathered}
\left(\int_{0}^{\underline{u}}\left|\partial_{\underline{u}} \phi\left(u, \underline{u}^{\prime}, \theta\right)\right|^{2} d \underline{u}^{\prime}\right)^{1 / 2} \lesssim\left(\left\|\partial_{\underline{u}}^{2} \phi\right\|_{L^{2}(H)}+\delta^{2}\|\phi\|_{L^{2}(H)}\right)^{1 / 2}\left\|\partial_{\theta}^{2} \phi\right\|_{L^{2}(H)}^{1 / 2} \\
+\left\|\partial_{\theta} \partial_{\underline{u}} \phi\right\|_{L^{2}(H)}+\delta\left\|\partial_{\theta} \phi\right\|_{L^{2}(H)}
\end{gathered}
$$

For any scalar function $\phi$ along $\underline{H}=\underline{H}_{\underline{u}}^{(0, u)}$, supported in a neighborhood patch,

$$
\begin{gathered}
\left(\int_{0}^{u}\left|\partial_{u} \phi\left(u^{\prime}, \underline{u}, \underline{\theta}\right)\right|^{2} d u^{\prime}\right)^{1 / 2} \lesssim\left(\left\|\partial_{u}^{2} \phi\right\|_{L^{2}(\underline{H})}+\left\|\partial_{u}^{2} \phi\right\|_{L^{2}(\underline{H})}\right)^{1 / 2}\left\|\partial_{\underline{\theta}}^{2} \phi\right\|_{L^{2}(\underline{H})}^{1 / 2} \\
+\left\|\partial_{\underline{\theta}} \partial_{u} \phi\right\|_{L^{2}(\underline{H})}+\left\|\partial_{\underline{\theta}} \phi\right\|_{L^{2}(\underline{H})} .
\end{gathered}
$$

In scale-invariant norms, we have

$$
\begin{gathered}
\left\|\partial_{\underline{u}} \phi\right\|_{\operatorname{Tr}_{(\mathrm{sc})}(H)} \lesssim\left(\left\|\partial_{\underline{u}}^{2} \phi\right\|_{\mathcal{L}_{\text {(sc) }}^{2}(H)}+\|\phi\|_{\mathcal{L}_{\text {(sc) }}^{2}(H)}\right)^{1 / 2}\left\|\partial_{\theta}^{2} \phi\right\|_{\mathcal{L}_{(\mathrm{sc})}^{2}(H)}^{1 / 2} \\
+\left\|\partial_{\theta} \partial_{\underline{u}} \phi\right\|_{\mathcal{L}_{(\mathrm{sc})}^{2}(H)}+\left\|\partial_{\theta} \phi\right\|_{\mathcal{L}_{(\mathrm{sc})}^{2}(H)}
\end{gathered}
$$

and

$$
\begin{gathered}
\left\|\partial_{u} \phi\right\|_{\operatorname{Tr}_{(\mathrm{sc})}(\underline{H})} \lesssim\left(\left\|\partial_{u}^{2} \phi\right\|_{\mathcal{L}_{(\mathrm{sc})}^{2}(\underline{H})}+\|\phi\|_{\mathcal{L}_{\text {(sc) }}^{2}(\underline{H})}\right)^{1 / 2}\left\|\partial_{\theta}^{2} \phi\right\|_{\mathcal{L}_{(\mathrm{sc})}^{2}(\underline{H})}^{1 / 2} \\
+\left\|\partial_{\theta} \partial_{\underline{u}} \phi\right\|_{\mathcal{L}_{(\mathrm{sc})}^{2}(\underline{H})}+\left\|\partial_{\theta} \phi\right\|_{\mathcal{L}_{(\mathrm{sc})}^{2}(\underline{H})}
\end{gathered}
$$


Proof. We start by making the additional assumption that $\phi(\underline{u}, \theta)$ is compactly supported for $\underline{u}^{\prime} \in(0, \underline{u})$.

Integrating by parts in $\theta=\left(\theta^{1}, \theta^{2}\right)$,

$$
\begin{aligned}
\left.\left|\int_{0}^{\underline{u}}\right| \partial_{\underline{u}} \phi\left(\underline{u}^{\prime}, \theta\right)\right|^{2} d \underline{u}^{\prime} \mid & =\left|\int_{\theta^{1}}^{\infty} \int_{\theta^{2}}^{\infty} \partial_{\theta^{1}} \partial_{\theta^{2}} \int_{0}^{\underline{u}} \partial_{\underline{u}} \phi\left(\underline{u}^{\prime}, \theta^{\prime}\right) \partial_{\underline{u}} \phi\left(\underline{u}^{\prime}, \theta^{\prime}\right) d \underline{u}^{\prime} d \theta^{\prime}\right| \\
\lesssim & \int_{D}\left|\partial_{\theta^{1}} \partial_{\theta^{2}} \int_{0}^{\underline{u}} \partial_{\underline{u}} \phi\left(\underline{u}^{\prime}, \theta^{\prime}\right) \partial_{\underline{u}} \phi\left(\underline{u}^{\prime}, \theta^{\prime}\right) d \underline{u}^{\prime}\right| d \theta^{\prime} \\
\lesssim & \int_{D}\left|\int_{0}^{\underline{u}} \partial_{\theta^{1}} \partial_{\theta^{2}} \partial_{\underline{u}} \phi\left(\underline{u}^{\prime}, \theta^{\prime}\right) \partial_{\underline{u}} \phi\left(\underline{u}^{\prime}, \theta^{\prime}\right) d \underline{u}^{\prime}\right| d \theta^{\prime} \\
& +\int_{D} \int_{0}^{\underline{u}}\left|\partial_{\theta} \partial_{\underline{u}} \phi\left(\underline{u}^{\prime}, \theta^{\prime}\right)\right|^{2} d \underline{u}^{\prime} d \theta^{\prime} .
\end{aligned}
$$

Now, integrating by parts in $\underline{u}$,

$$
\int_{0}^{\underline{u}} \partial_{\theta^{1}} \partial_{\theta^{2}} \partial_{\underline{u}} \phi\left(\underline{u}^{\prime}, \theta\right) \partial_{\underline{u}} \phi\left(\underline{u}^{\prime}, \theta\right) d \underline{u}^{\prime}=-\int_{0}^{\underline{u}} \partial_{\theta^{1}} \partial_{\theta^{2}} \phi\left(\underline{u}^{\prime}, \theta\right) \partial_{\underline{u}}^{2} \phi\left(\underline{u}^{\prime}, \theta\right) d \underline{u}^{\prime} .
$$

Hence,

$$
\int_{0}^{\underline{u}}\left|\partial_{\underline{u}} \phi(\underline{u}, \theta)\right|^{2} d \underline{u}^{\prime} \lesssim\left\|\partial_{\theta}^{2} \phi\right\|_{L^{2}(H)}\left\|\partial_{\underline{u}}^{2} \phi\right\|_{L^{2}(H)}+\left\|\partial_{\theta} \partial_{\underline{u}} \phi\right\|_{L^{2}(H)}^{2} .
$$

To remove our additional assumption concerning the compact support in $(0, \underline{u})$, we simply extend the original $\phi$ to $-\delta \leqslant \underline{u} \leqslant 2 \delta$ in such a way that all norms on the right-hand side of (11.3), on the extended interval, are bounded by a constant multiple of the same norms restricted to the original interval $(0, \underline{u})$. We then apply a cut-off to make the extended $\phi$ compactly supported in the interval $(-\delta, 2 \delta)$, and finally use $(11.5)$ in the extended interval to get the desired result. The proof of (11.4) is exactly the same. The scale version of these estimates is immediate.

We now pass to the proof of Proposition 11.1. It suffices to prove (11.1), the proof of (11.2) is exactly the same.

One can easily pass from the coordinate-dependent form of the trace inequalities to a covariant form with the help of the estimates of Proposition 4.4.

According to that proposition we have, for $C=C\left(\mathcal{O}^{(0)}, \mathcal{R}, \underline{\mathcal{R}}\right)$,

$$
\|\Gamma\|_{\mathcal{L}_{(\mathrm{sc})}^{2}(S)}+\|\nabla \Gamma\|_{\mathcal{L}_{(\mathrm{sc})}^{2}(S)} \lesssim C
$$

Thus,

$$
\nabla_{4} \phi_{a}=\Omega^{-1} \partial_{\underline{u}} \phi_{a}-\chi_{a b} \phi_{b} .
$$

As a consequence, along $H=H_{u}$,

$$
\begin{aligned}
\left\|\nabla_{4} \phi\right\|_{\operatorname{Tr}_{(\mathrm{sc})}(H)} & \lesssim\left\|\partial_{\underline{u}} \phi\right\|_{\operatorname{Tr}_{(\mathrm{sc})}(H)}+\delta^{1 / 2}\|\chi\|_{\mathcal{L}_{\text {(sc) }}^{\infty}\|\phi\|_{\mathcal{L}_{\text {(sc) }}^{\infty}}} \\
& \lesssim\left\|\partial_{\underline{u}} \phi\right\|_{\operatorname{Tr}_{\text {(sc) }}(H)}+C \delta^{1 / 2}\|\phi\|_{\mathcal{L}_{\text {(sc) }}^{\infty}} .
\end{aligned}
$$


Also, schematically, ignoring factors of $\Omega$ (which are bounded in $L^{\infty}$ ), we have, with $\psi \in\{\chi, \omega\}$,

$$
\nabla_{4}^{2} \phi=\partial_{\underline{u}}^{2} \phi+\psi \partial_{\underline{u}} \phi+\alpha \phi+\psi \psi \phi
$$

Thus, in view of our estimates for the Ricci coefficients $\psi$, we have

$$
\begin{aligned}
\left\|\partial_{\underline{u}}^{2} \phi\right\|_{\mathcal{L}_{(\mathrm{sc})}^{2}(H)} \lesssim\left\|\nabla_{4}^{2} \phi\right\|_{\mathcal{L}_{(\mathrm{sc})}^{2}(H)}+\delta^{1 / 2}\|\psi\|_{\mathcal{L}_{(\mathrm{sc})}^{\infty}}\left\|\nabla_{4} \phi\right\|_{\mathcal{L}_{(\mathrm{sc})}^{2}(H)} \\
+\delta^{1 / 2}\|\phi\|_{\mathcal{L}_{(\mathrm{sc})}^{\infty}}\left(\|\alpha\|_{\mathcal{L}_{(\mathrm{sc})}^{2}(H)}+\|\psi\|_{\mathcal{L}_{(\mathrm{sc})}^{\infty}}^{2}\right) \\
\lesssim\left\|\nabla_{4}^{2} \phi\right\|_{\mathcal{L}_{(\mathrm{sc})}^{2}(H)}+C \delta^{1 / 2}\left(\left\|\nabla_{4} \phi\right\|_{\mathcal{L}_{(\mathrm{sc})}^{2}(H)}+\|\phi\|_{\left.\mathcal{L}_{(\mathrm{sc})}^{\infty}\right)}\right)
\end{aligned}
$$

We next note that for a horizontal tensor we can convert $\partial_{\theta}$ into a covariant $\nabla$ derivative according to the formula $\partial_{\theta}=\nabla+\Gamma$. Therefore,

$$
\left\|\partial_{\theta} \phi_{a}\right\|_{\mathcal{L}_{(\mathrm{sc})}^{2}(S)} \lesssim\|\nabla \phi\|_{\mathcal{L}_{(\mathrm{sc})}^{2}(S)}+\delta^{1 / 2}\|\Gamma\|_{\mathcal{L}_{(\mathrm{sc})}^{2}(S)}\|\phi\|_{\mathcal{L}_{(\mathrm{sc})}^{\infty}} \lesssim\|\nabla \phi\|_{\mathcal{L}_{(\mathrm{sc})}^{2}(S)}+\delta^{1 / 2} C\|\phi\|_{\mathcal{L}_{(\mathrm{sc})}^{\infty}}
$$

and

$$
\begin{aligned}
\left\|\partial_{\theta}^{2} \phi_{a}\right\|_{\mathcal{L}_{(\mathrm{sc})}^{2}(S)} & \lesssim\left\|\nabla^{2} \phi\right\|_{\mathcal{L}_{(\mathrm{sc})}^{2}(S)}+\delta^{1 / 2}\|\partial \Gamma\|_{\mathcal{L}_{(\mathrm{sc})}^{2}(S)}\|\phi\|_{\mathcal{L}_{(\mathrm{sc})}^{\infty}}+\delta^{1 / 2}\|\Gamma\|_{\mathcal{L}_{(\mathrm{sc})}^{4}(S)}\|\nabla \phi\|_{\mathcal{L}_{(\mathrm{sc})}^{4}(S)} \\
& \lesssim\left\|\nabla^{2} \phi\right\|_{\mathcal{L}_{(\mathrm{sc})}^{2}(S)}+\delta^{1 / 2} C\left(\|\phi\|_{\mathcal{L}_{(\mathrm{sc})}^{\infty}}+\|\nabla \phi\|_{\mathcal{L}_{(\mathrm{sc})}^{4}(S)}\right) .
\end{aligned}
$$

Also,

$$
\begin{aligned}
\left\|\partial_{\theta} \partial_{\underline{u}} \phi_{a}\right\|_{\mathcal{L}_{(\mathrm{sc})}^{2}(S)} & \lesssim\left\|\nabla \nabla_{4} \phi\right\|_{L^{2}(S)}+\delta^{1 / 2}\|\partial \Gamma\|_{L^{2}(S)}\|\phi\|_{L^{\infty}}+\delta^{1 / 2}\|\Gamma\|_{L^{4}(S)}\left\|\nabla_{4} \phi\right\|_{L^{4}(S)} \\
& \lesssim\left\|\nabla \nabla_{4} \phi\right\|_{\mathcal{L}_{(\mathrm{sc})}^{2}(S)}+\delta^{1 / 2} C\left(\|\phi\|_{\mathcal{L}_{(\mathrm{sc})}^{\infty}}+\left\|\nabla_{4} \phi\right\|_{\mathcal{L}_{(\mathrm{sc})}^{4}(S)}\right) .
\end{aligned}
$$

According to the scale-invariant estimate of Lemma 11.2,

$$
\begin{gathered}
\left.\left\|\partial_{\underline{u}} \phi\right\|_{\operatorname{Tr}_{(\mathrm{sc})}(H) \lesssim(}\left\|\partial_{\underline{u}}^{2} \phi\right\|_{\mathcal{L}_{(\mathrm{sc})}^{2}(H)}+\|\phi\|_{\mathcal{L}_{(\mathrm{sc})}^{2}(H)}\right)^{1 / 2}\left\|\partial_{\theta}^{2} \phi\right\|_{\mathcal{L}_{(\mathrm{sc})}^{2}(H)}^{1 / 2} \\
+\left\|\partial_{\theta} \partial_{\underline{u}} \phi\right\|_{\mathcal{L}_{(\mathrm{sc})}^{2}(H)}+\left\|\partial_{\theta} \phi\right\|_{\mathcal{L}_{(\mathrm{sc})}^{2}(H)} .
\end{gathered}
$$

Combining this with the previous estimates, we obtain the desired result, which can be clearly extended to any $\phi$ along $H_{u}$, not necessarily restricted to a coordinate patch, by a simple partition-of-unity argument. This proves the desired estimate (11.1). Estimate (11.2) is proved in exactly the same manner.

\subsection{Estimate for the trace norms of $\nabla \chi$ and $\nabla \underline{\chi}$}

Our main goal in this subsection is to derive estimates for the trace norms $\|\nabla \chi\|_{\operatorname{Tr}(\mathrm{sc})}(H)$

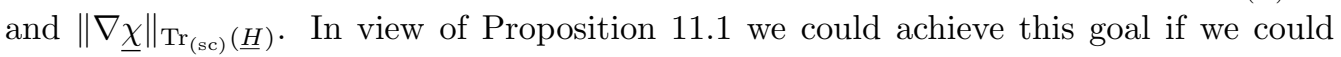
write $\nabla \widehat{\chi}=\nabla_{4} \phi$ and $\nabla \underline{\hat{\chi}}=\nabla_{3} \underline{\phi}$, where $\phi$ and $\underline{\phi}$ are such that the norms on the right-hand sides of (11.1) and (11.2), respectively, are finite. We prove the following proposition. 
Proposition 11.3. Consider the following transport equations along $H=H_{u}$, resp. $\underline{H}=\underline{H}_{u}$,

$$
\nabla_{4} \phi=\nabla \widehat{\chi}, \quad \phi(0, \underline{u})=0
$$

resp.

$$
\nabla_{3} \underline{\phi}=\nabla \underline{\hat{\chi}}, \quad \underline{\phi}(0, \underline{u})=0 .
$$

(1) The solution $\phi$ of (11.6) satisfies the estimates

$$
\begin{array}{r}
\|\phi\|_{\mathcal{L}_{(\mathrm{sc})}^{2}(S)}+\|\phi\|_{\mathcal{L}_{(\mathrm{sc})}^{4}(S)}+\|\nabla \phi\|_{\mathcal{L}_{(\mathrm{sc})}^{2}(S)}+\left\|\nabla_{4} \phi\right\|_{\mathcal{L}_{(\mathrm{sc})}^{2}(S)} \lesssim C, \\
\left\|\nabla \nabla_{4} \phi\right\|_{\mathcal{L}_{(\mathrm{sc})}^{2}(H)}+\left\|\nabla_{4}^{2} \phi\right\|_{\mathcal{L}_{(\mathrm{sc})}^{2}(H)} \lesssim C,
\end{array}
$$

with a constant $C=C\left(\mathcal{O}^{(0)}, \mathcal{R}, \underline{\mathcal{R}}\right)$. Moreover,

$$
\left\|\nabla^{2} \phi\right\|_{\mathcal{L}_{(\mathrm{sc})}^{2}(H)} \lesssim\left\|\nabla^{3} \operatorname{tr} \chi\right\|_{\mathcal{L}_{(\mathrm{sc})}^{2}(H)}+C
$$

As a consequence (see the calculus inequalities of $\S 4.4$ ) we also have

$$
\|\phi\|_{\mathcal{L}_{(\mathrm{sc})}^{\infty}} \lesssim\left\|\nabla^{3} \operatorname{tr} \chi\right\|_{\mathcal{L}_{(\mathrm{sc})}^{2}(H)}+C
$$

and, as a consequence of the trace estimate (11.1),

$$
\left\|\nabla_{4} \phi\right\|_{\operatorname{Tr}_{(\mathrm{sc})}(H)} \lesssim\left\|\nabla^{3} \operatorname{tr} \chi\right\|_{\mathcal{L}_{(\mathrm{sc})}^{2}(H)}+C .
$$

(2) The solution $\phi$ of (11.7) satisfies the estimates

$$
\begin{array}{r}
\|\underline{\phi}\|_{\mathcal{L}_{(\mathrm{sc})}^{2}(S)}+\|\underline{\phi}\|_{\mathcal{L}_{(\mathrm{sc})}^{4}(S)}+\|\nabla \underline{\phi}\|_{\mathcal{L}_{(\mathrm{sc})}^{2}(S)}+\left\|\nabla_{3} \underline{\phi}\right\|_{\mathcal{L}_{(\mathrm{sc})}^{2}(S)} \lesssim C, \\
\left\|\nabla \nabla_{3} \underline{\phi}\right\|_{\mathcal{L}_{(\mathrm{sc})}^{2}(\underline{H})}+\left\|\nabla_{3}^{2} \phi\right\|_{\mathcal{L}_{(\mathrm{sc})}^{2}(\underline{H})} \lesssim C,
\end{array}
$$

with a constant $C=C\left(\mathcal{O}^{(0)}, \mathcal{R}, \underline{\mathcal{R}}\right)$. Moreover,

$$
\left\|\nabla^{2} \underline{\phi}\right\|_{\mathcal{L}_{(\mathrm{sc})}^{2}(\underline{H})} \lesssim\left\|\nabla^{3} \operatorname{tr} \chi\right\|_{\mathcal{L}_{(\mathrm{sc})}^{2}(\underline{H})}+C .
$$

As a consequence (see the calculus inequalities of §4.4) we also have

$$
\|\underline{\phi}\|_{\mathcal{L}_{(\mathrm{sc})}^{\infty}} \lesssim\left\|\nabla^{3} \operatorname{tr} \underline{\chi}\right\|_{\mathcal{L}_{(\mathrm{sc})}^{2}(\underline{H})}+C
$$

and, as a consequence of the trace estimate (11.1),

$$
\left\|\nabla_{3} \underline{\phi}\right\|_{\operatorname{Tr}_{(\mathrm{sc})}(\underline{H})} \lesssim\left\|\nabla^{3} \operatorname{tr} \underline{\chi}\right\|_{\mathcal{L}_{(\mathrm{sc})}^{2}(H)}+C .
$$


Proof. Estimates (11.8) and (11.9), resp. (11.11) and (11.12), follow easily from (11.6), resp. (11.7), in view of our estimates for $\widehat{\chi}$, resp. $\underline{\hat{\chi}}$, and their first two derivatives derived in the previous sections. The second $\nabla$ derivative estimates are subtle; they require a non-trivial renormalization procedure, nothing less than another series of miracles. As always we expect the estimates for $\phi$ to be somewhat more demanding in view of the presence of $\operatorname{tr} \underline{\chi}=\operatorname{tr} \chi_{0}+\widetilde{\operatorname{tr} \underline{\chi}}$. We shall thus concentrate on them in what follows. No other anomalies occur at this high level of differentiability. The idea is to first derive a transport equation for $\Delta \phi$ and hope somehow that the principal term on the right, i.e. $\nabla \Delta \underline{\hat{\chi}}$, can be re-expressed as a $\nabla_{3}$ derivative of another quantity depending only on two derivatives of a Ricci coefficient. We write

$$
\nabla_{3} \Delta \underline{\phi}=\Delta \nabla \underline{\hat{\chi}}+\left[\nabla_{3}, \Delta\right] \phi
$$

Now, recalling commutation Lemma 3.1 , we write schematically (we eliminate $\underline{\beta}$ using the Codazzi equation)

$$
\begin{aligned}
{\left[\nabla_{3}, \nabla\right] \underline{\phi}=} & \underline{\chi} \nabla \phi+\left(\nabla \psi_{3}\right) \phi+\psi_{3} \nabla_{3} \phi+\underline{\chi} \psi_{3} \phi \\
{\left[\nabla_{3}, \nabla^{2}\right] \underline{\phi}=} & \underline{\chi} \nabla^{2} \phi+\left(\nabla \psi_{3}\right)\left(\nabla \phi+\nabla_{3} \phi\right)+\left(\nabla^{2} \psi_{3}\right) \phi+\psi_{3} \nabla \nabla_{3} \phi+\nabla\left(\underline{\widehat{\chi}} \psi_{3} \phi\right) \\
& \quad+\psi_{3} \nabla_{3} \nabla \phi+\underline{\widehat{\chi}} \psi_{3} \nabla \phi
\end{aligned}
$$

where $\psi_{3} \in\{\eta, \widetilde{\operatorname{tr} \underline{\chi}}, \underline{\hat{\chi}}, \underline{\eta}\}$.

Hence, using our estimates for $\psi_{3}$ as well as the estimates (11.11) and (11.12) for $\phi$, we can write

$$
\begin{aligned}
& {\left[\nabla_{3}, \Delta\right] \underline{\phi} }=\left(\operatorname{tr} \underline{\chi}_{0}\right) \nabla^{2} \underline{\phi}+\underline{\widehat{\chi}} \nabla^{2} \underline{\phi}+\operatorname{Err}_{\phi} \\
&\left\|\operatorname{Err}_{\phi}\right\|_{\mathcal{L}_{(\mathrm{sc})}^{2}(\underline{H})} \lesssim C \delta^{1 / 2}\left(C+\left\|\nabla^{2} \underline{\phi}\right\|_{\mathcal{L}_{(\mathrm{sc})}^{2}(\underline{H})}\right)
\end{aligned}
$$

Indeed we have, for example,

$$
\begin{aligned}
\left\|\nabla^{2} \psi_{3} \underline{\phi}\right\|_{\mathcal{L}_{(\mathrm{sc})}^{2}(\underline{H})} & \lesssim \delta^{1 / 2}\|\underline{\phi}\|_{\mathcal{L}_{(\mathrm{sc})}^{\infty}}\left\|\nabla^{2} \psi_{3}\right\|_{\mathcal{L}_{(\mathrm{sc})}^{2}(\underline{H})} \lesssim \delta^{1 / 2} C\|\underline{\phi}\|_{\mathcal{L}_{(\mathrm{sc})}^{\infty}} \\
& \lesssim C \delta^{1 / 2}\left(\left\|\nabla^{2} \phi\right\|_{\mathcal{L}_{(\mathrm{sc})}^{2}(\underline{H})}+\left\|\nabla \nabla_{3} \underline{\phi}\right\|_{\mathcal{L}_{(\mathrm{sc})}^{2}(\underline{H})}+\|\phi\|_{\mathcal{L}_{(\mathrm{sc})}^{2}(\underline{H})}\right) \\
& \lesssim C \delta^{1 / 2}\left\|\nabla^{2} \phi\right\|_{\mathcal{L}_{(\mathrm{sc})}^{2}(\underline{H})}+C^{2} \delta^{1 / 2}
\end{aligned}
$$

Consequently,

$$
\nabla_{3} \Delta \underline{\phi}=\Delta \nabla \underline{\hat{\chi}}+\left(\operatorname{tr} \underline{\chi}_{0}\right) \nabla^{2} \underline{\phi}+\underline{\widehat{\chi}} \nabla^{2} \underline{\phi}+\operatorname{Err}_{\phi} .
$$

Since

$$
[\Delta, \nabla] \phi=K \nabla \phi+\nabla K \phi
$$


we have

$$
\begin{aligned}
\|[\Delta, \nabla] \phi\|_{\mathcal{L}_{(\mathrm{sc})}^{2}(\underline{H})} & \lesssim\|K\|_{\mathcal{L}_{(\mathrm{sc})}^{4}(\underline{H})}\|\nabla \phi\|_{\mathcal{L}_{(\mathrm{sc})}^{4}(\underline{H})}+\|\nabla K\|_{\mathcal{L}_{(\mathrm{sc})}^{2}(\underline{H})}\|\phi\|_{\mathcal{L}_{(\mathrm{sc})}^{\infty}} \\
& \lesssim C \delta^{1 / 2}\left\|\nabla^{2} \phi\right\|_{\mathcal{L}_{(\mathrm{sc})}^{2}(\underline{H})}+C^{2} \delta^{1 / 2}
\end{aligned}
$$

Hence, also,

$$
\begin{aligned}
\nabla_{3} \Delta \underline{\phi} & =\Delta \nabla \underline{\hat{\chi}}+\left(\operatorname{tr} \underline{\chi}_{0}\right) \nabla^{2} \underline{\phi}+\underline{\hat{\chi}} \nabla^{2} \underline{\phi}+E, \\
\|E\|_{\mathcal{L}_{(\mathrm{sc})}^{2}(\underline{H})} & \lesssim C \delta^{1 / 2}\left(C+\left\|\nabla^{2} \underline{\phi}\right\|_{\mathcal{L}_{(\mathrm{sc})}^{2}(\underline{H})}\right) .
\end{aligned}
$$

Now, according to the Codazzi equations,

$$
\mathcal{D}_{2} \underline{\widehat{\chi}}=-\underline{\beta}-\frac{1}{2} \nabla \operatorname{tr} \underline{\chi}+(\operatorname{tr} \underline{\chi}) \psi_{3}+\psi_{3} \psi_{3}
$$

Thus,

$$
{ }^{\star} \mathcal{D}_{2} \mathcal{D}_{2} \underline{\widehat{\chi}}={ }^{\star} \mathcal{D}_{2} \underline{\beta}-\frac{1}{2}{ }^{\star} \mathcal{D}_{2} \nabla \operatorname{tr} \underline{\chi}+{ }^{\star} \mathcal{D}_{2}\left((\operatorname{tr} \underline{\chi}) \psi_{3}+\psi_{3} \psi_{3}\right)
$$

or, making use of (3.12),

$$
-\frac{1}{2} \Delta \underline{\hat{\chi}}+K \underline{\hat{\chi}}={ }^{\star} \mathcal{D}_{2} \underline{\beta}-\frac{1}{2}{ }^{\star} \mathcal{D}_{2} \nabla \operatorname{tr} \chi+{ }^{\star} \mathcal{D}_{2}\left((\operatorname{tr} \underline{\chi}) \psi_{3}+\psi_{3} \psi_{3}\right) .
$$

Thus, differentiating once more,

$$
\begin{aligned}
\nabla \Delta \underline{\hat{\chi}} & =\nabla^{2} \underline{\beta}+\nabla^{3} \operatorname{tr} \underline{\chi}+K \nabla \underline{\hat{\chi}}+\operatorname{Err}, \\
\operatorname{Err} & =(\nabla K) \underline{\hat{\chi}}+(\operatorname{tr} \underline{\chi}) \nabla^{2} \psi_{3}+\nabla^{2}\left(\psi_{3} \psi_{3}\right) .
\end{aligned}
$$

Here, and in what follows, Err denotes an error term such that

$$
\|\operatorname{Err}\|_{\mathcal{L}_{(\mathrm{sc})}^{2}(\underline{H})} \lesssim C
$$

On the other hand, we recall the structure equation

$$
\nabla_{3} \underline{\eta}=\underline{\beta}+\underline{\chi}(\eta-\underline{\eta})
$$

Thus, commuting and writing as before,

$$
\begin{aligned}
{\left[\nabla_{3}, \nabla\right] \underline{\eta}=} & \underline{\chi} \nabla \underline{\eta}+\left(\nabla \psi_{3}\right) \underline{\eta}+\psi_{3} \nabla_{3} \underline{\eta}+\underline{\chi} \psi_{3} \underline{\eta}, \\
{\left[\nabla_{3}, \nabla^{2}\right] \underline{\eta}=} & \underline{\chi} \nabla^{2} \underline{\eta}+\left(\nabla \psi_{3}\right)\left(\nabla \underline{\eta}+\nabla_{3} \underline{\eta}\right)+\left(\nabla^{2} \psi_{3}\right) \underline{\eta}+\psi_{3} \nabla \nabla_{3} \underline{\eta}+\nabla\left(\underline{\widehat{\chi}} \psi_{3} \underline{\eta}\right) \\
& +\psi_{3} \nabla_{3} \nabla \underline{\eta}+\underline{\widehat{\chi}} \psi_{3} \nabla \underline{\eta} .
\end{aligned}
$$


Observe that

$$
\left\|\left[\nabla_{3}, \nabla\right] \underline{\eta}\right\|_{\mathcal{L}_{(\mathrm{sc})}^{2}(\underline{H})} \lesssim C
$$

and, consequently,

$$
\begin{aligned}
\nabla^{2} \underline{\beta} & =\nabla_{3} \nabla^{2} \underline{\eta}+\text { Err }, \\
\operatorname{Err} & =\nabla^{2}(\underline{\chi}(\eta-\underline{\eta}))+\left[\nabla_{3}, \nabla^{2}\right] \underline{\eta} .
\end{aligned}
$$

Clearly,

$$
\|\operatorname{Err}\|_{\mathcal{L}_{(\mathrm{sc})}^{2}(\underline{H})} \lesssim C .
$$

Therefore, we deduce that

$$
\nabla \Delta \underline{\hat{\chi}}=\nabla_{3} \nabla^{2} \underline{\eta}+\nabla^{3} \operatorname{tr} \underline{\chi}+K \nabla \underline{\hat{\chi}}+\operatorname{Err} .
$$

Commuting $\nabla$ with $\Delta$ again,

$$
\Delta \nabla \underline{\hat{\chi}}=\nabla \Delta \underline{\hat{\chi}}+K \nabla \underline{\hat{\chi}}+(\nabla K) \underline{\hat{\chi}} .
$$

Hence, since $\nabla \underline{\widehat{\chi}}=\nabla_{3} \underline{\phi}$,

$$
\Delta \nabla \underline{\hat{\chi}}=\nabla_{3} \nabla^{2} \underline{\eta}+\nabla^{3} \operatorname{tr} \underline{\chi}+K \nabla_{3} \underline{\phi}+\operatorname{Err} .
$$

Back to (11.18), we rewrite

$$
\begin{aligned}
\nabla_{3} \Delta \underline{\phi} & =-\nabla_{3} \nabla^{2} \underline{\eta}+\nabla^{3} \operatorname{tr} \underline{\chi}+\left(\operatorname{tr} \underline{\chi}_{0}\right) \nabla^{2} \underline{\phi}+K \nabla_{3} \underline{\phi}+\operatorname{Err}_{\phi}, \\
\left\|\operatorname{Err}_{\phi}\right\|_{\mathcal{L}_{\text {(sc) }}^{2}(\underline{H})} & \lesssim C\left(1+\delta^{1 / 2}\left\|\nabla^{2} \underline{\phi}\right\|_{\mathcal{L}_{\text {(sc) }}^{2}(\underline{H})}\right),
\end{aligned}
$$

which we could rewrite in the form

$$
\nabla_{3}\left(\Delta \underline{\phi}+\nabla^{2} \underline{\eta}-K \underline{\phi}\right)=\nabla^{3} \operatorname{tr} \underline{\chi}+\left(\operatorname{tr} \underline{\chi}_{0}\right) \nabla^{2} \phi-\left(\nabla_{3} K\right) \underline{\phi}+\operatorname{Err}_{\phi}
$$

Recall that $K=\varrho-\frac{1}{4}(\operatorname{tr} \chi) \operatorname{tr} \underline{\chi}-\frac{1}{2} \hat{\chi} \underline{\hat{\chi}}$. Hence, we easily find

$$
\left\|\nabla_{3} K\right\|_{\mathcal{L}_{(\mathrm{sc})}^{2}(\underline{H})} \lesssim C
$$

Thus,

$$
\left\|\nabla_{3}\left(\Delta \underline{\phi}+\nabla^{2} \underline{\eta}-K \underline{\hat{\chi}}\right)\right\|_{\mathcal{L}_{(\mathrm{sc})}^{2}(u, \underline{u})} \lesssim\left\|\nabla^{3} \operatorname{tr} \underline{\chi}\right\|_{\mathcal{L}_{(\mathrm{sc})}^{2}(u, \underline{u})}+\left\|\nabla^{2} \underline{\phi}\right\|_{\mathcal{L}_{(\mathrm{sc})}^{2}(u, \underline{u})}+\left\|\operatorname{Err}_{\phi}\right\|_{\mathcal{L}_{(\mathrm{sc})}^{2}(u, \underline{u})}
$$


i.e.,

$$
\begin{aligned}
\|\Delta \underline{\phi}\|_{\mathcal{L}_{(\mathrm{sc})}^{2}(u, \underline{u})} \lesssim\left\|\nabla^{2} \underline{\eta}\right\|_{\mathcal{L}_{\text {(sc) }}^{2}(u, \underline{u})}+C \delta^{1 / 2}\|K\|_{\mathcal{L}_{\text {(sc) }}^{2}(u, \underline{u})}+\left\|\nabla^{3} \operatorname{tr} \underline{\chi}\right\|_{\mathcal{L}_{\text {(sc) }}^{2}(\underline{H})} \\
+\left(1+\delta^{1 / 2} C\right) \int_{0}^{u}\left\|\nabla^{2} \underline{\phi}\right\|_{\mathcal{L}_{\text {(sc) }}^{2}\left(u^{\prime}, \underline{u}\right)} d u^{\prime}+\left\|\operatorname{Err}_{\phi}\right\|_{\mathcal{L}_{\text {(sc) }}^{2}(\underline{H})} .
\end{aligned}
$$

Now, using the elliptic estimates discussed in $\S 4.6$ and our estimates for $K$, we deduce that

$$
\begin{aligned}
& \left\|\nabla^{2} \underline{\phi}\right\|_{\mathcal{L}_{\text {(sc) }}^{2}(S)} \lesssim\|\Delta \underline{\phi}\|_{\mathcal{L}_{\text {(sc) }}^{2}(S)}+\delta^{1 / 2}\left(\|\nabla K\|_{\mathcal{L}_{\text {(sc) }}^{2}(S)}\|\underline{\phi}\|_{\mathcal{L}_{\text {(sc) }}^{\infty}(S)}+\|K\|_{\mathcal{L}_{(\mathrm{sc})}^{4}(S)}\|\nabla \underline{\phi}\|_{\mathcal{L}_{\text {(sc) }}^{4}(S)}\right) \\
& \lesssim\|\Delta \underline{\phi}\|_{\mathcal{L}_{(\mathrm{sc})}^{2}(S)}+\delta^{1 / 2}\left(\|\underline{\phi}\|_{\mathcal{L}_{(\mathrm{sc})}^{\infty}(S)}+\|\nabla \underline{\phi}\|_{\mathcal{L}_{(\mathrm{sc})}^{4}(S)}\right) \\
& \lesssim\|\Delta \underline{\phi}\|_{\mathcal{L}_{(\mathrm{sc})}^{2}(S)}+\delta^{1 / 2}\left(C+\left\|\nabla^{2} \underline{\phi}\right\|_{\mathcal{L}_{(\mathrm{sc})}^{2}(\underline{H})}\right) \text {. }
\end{aligned}
$$

Thus,

$$
\begin{aligned}
\left\|\nabla^{2} \underline{\phi}\right\|_{\mathcal{L}_{\text {(sc) }}^{2}(u, \underline{u})} \lesssim\left\|\nabla^{2} \underline{\eta}\right\|_{\mathcal{L}_{\text {(sc) }}^{2}(u, \underline{u})}+C \delta^{1 / 2}\|K\|_{\mathcal{L}_{(\mathrm{sc})}^{2}(u, \underline{u})}+\left\|\nabla^{3} \operatorname{tr} \underline{\chi}\right\|_{\mathcal{L}_{\text {(sc) }}^{2}(\underline{H})} \\
+\left(1+\delta^{1 / 2} C\right) \int_{0}^{u}\left\|\nabla^{2} \underline{\phi}\right\|_{\mathcal{L}_{(\mathrm{sc})}^{2}\left(u^{\prime}, \underline{u}\right)} d u^{\prime}+C\left(1+\delta^{1 / 2}\right) \| \nabla^{2} \underline{\|_{\mathcal{L}_{(\mathrm{sc})}(\underline{H})}} .
\end{aligned}
$$

Using Grönwall's inequality,

$$
\begin{gathered}
\left\|\nabla^{2} \underline{\phi}\right\|_{\mathcal{L}_{(\mathrm{sc})}^{2}(u, \underline{u})} \lesssim\left\|\nabla^{2} \underline{\underline{\eta}}\right\|_{\mathcal{L}_{(\mathrm{sc})}^{2}(u, \underline{u})}+C \delta^{1 / 2}\|K\|_{\mathcal{L}_{(\mathrm{sc})}^{2}(u, \underline{u})}+\left\|\nabla^{3} \operatorname{tr} \underline{\chi}\right\|_{\mathcal{L}_{(\mathrm{sc})}^{2}(\underline{H})} \\
+C\left(1+\delta^{1 / 2}\right)\left\|\nabla^{2} \underline{\phi}\right\|_{\mathcal{L}_{(\mathrm{sc})}^{2}(\underline{H})} .
\end{gathered}
$$

Integrating, we deduce, for $C \delta^{1 / 2}$ sufficiently small, that

$$
\left\|\nabla^{2} \underline{\phi}\right\|_{\mathcal{L}_{(\mathrm{sc})}^{2}(\underline{H})} \lesssim C+\left\|\nabla^{3} \operatorname{tr} \underline{\chi}\right\|_{\mathcal{L}_{\text {(sc) }}^{2}(\underline{H})}
$$

as desired.

To close the estimates of Proposition 11.3 it remains to estimate $\left\|\nabla^{3} \operatorname{tr} \chi\right\|_{\mathcal{L}_{(\mathrm{sc})}^{2}(H)}$ and $\left\|\nabla^{3} \operatorname{tr} \underline{\chi}\right\|_{\mathcal{L}_{\text {(sc) }}^{2}(\underline{H})}$. To achieve this, we start with the transport equation for tr $\underline{\chi}$,

$$
\nabla_{3} \operatorname{tr} \underline{\chi}=-\frac{1}{2}(\operatorname{tr} \underline{\chi})^{2}-|\underline{\hat{\chi}}|^{2}-2 \underline{\omega} \operatorname{tr} \underline{\chi}
$$

which we rewrite in the form

$$
\begin{aligned}
\nabla_{3} \operatorname{tr} \underline{\chi}^{\prime} & =-\frac{1}{2} \Omega^{-1}(\operatorname{tr} \underline{\chi})^{2}-\Omega^{-1}|\underline{\hat{\chi}}|^{2}, \\
\operatorname{tr} \underline{\chi^{\prime}} & =\Omega^{-1} \operatorname{tr} \underline{\chi} .
\end{aligned}
$$


The plan is to derive a transport equation for the quantity $\Delta \nabla \operatorname{tr} \underline{\chi}^{\prime}$. We make use of the following commutation formulas, written schematically, for an arbitrary scalar $f$ satisfying the equation $\nabla_{3} f=F$,

$$
\begin{aligned}
& \nabla_{3} \nabla f=\nabla F+\underline{\chi} \nabla f+\psi_{3} F \\
& \nabla_{3} \nabla^{2} f\left.=\nabla\left(\nabla F+\underline{\chi} \nabla f+\psi_{3} F\right)+\underline{\chi} \nabla^{2} f+\underline{\beta} \nabla f+\psi_{3} \nabla_{3} \nabla f\right) \\
&= \nabla^{2} F+\psi_{3} \nabla F+\left(\nabla \psi_{3}\right) F+\underline{\chi} \nabla^{2} f+(\nabla \underline{\chi}) \nabla f+\psi_{3} \nabla_{3} \nabla f+\underline{\chi} \psi_{3} \nabla f, \\
& \nabla_{3} \nabla^{3} f=\nabla^{3} F+\psi_{3} \nabla^{2} F+\left(\nabla \psi_{3}\right) \nabla F+\left(\nabla^{2} \psi_{3}\right) F+\underline{\chi} \nabla^{3} f+(\nabla \underline{\chi}) \nabla^{2} f+\left(\nabla^{2} \underline{\chi}\right) \nabla f \\
& \quad+\nabla\left(\psi_{3} \nabla_{3} \nabla f+\underline{\widehat{\chi}} \psi_{3} \nabla f\right)+\underline{\beta} \nabla^{2} f+\psi_{3} \nabla_{3} \nabla^{2} f,
\end{aligned}
$$

that is

$$
\begin{gathered}
\nabla_{3} \nabla^{3} f=\nabla^{3} F+\psi_{3} \nabla^{2} F+\left(\nabla \psi_{3}\right) \nabla F+\left(\nabla^{2} \psi_{3}\right) F+\underline{\chi} \nabla^{3} f+(\nabla \underline{\chi}) \nabla^{2} f+\left(\nabla^{2} \underline{\chi}\right) \nabla f \\
+\psi_{3} \nabla_{3} \nabla^{2} f+\left(\nabla \psi_{3}\right) \nabla_{3} \nabla f+\psi_{3}\left[\nabla, \nabla_{3}\right] \nabla f+\nabla\left(\underline{\widehat{\chi}} \psi_{3} \nabla f\right)+\underline{\beta} \nabla^{2} f .
\end{gathered}
$$

Applying the calculations above to $f=\Omega^{-1} \operatorname{tr} \chi$ and $F=-\frac{1}{2} \Omega^{-1}(\operatorname{tr} \chi)^{2}-\Omega^{-1}|\chi|^{2}$, and using $\nabla\left(\Omega^{-1}\right)=-\Omega^{-2} \nabla \Omega=-\frac{1}{2} \Omega^{-2}(\eta-\underline{\eta})$ we derive, omitting factors of $\Omega$ which are bounded in $L^{\infty}$, that

$$
\begin{aligned}
& \nabla_{3} \Delta \nabla \operatorname{tr} \underline{\chi}^{\prime}=\underline{\hat{\chi}} \Delta \nabla \underline{\hat{\chi}}+\underline{\chi} \nabla^{3} \operatorname{tr} \underline{\chi}+(\nabla \underline{\hat{\chi}}) \nabla^{2} \underline{\hat{\chi}}+(\nabla \underline{\chi}) \nabla^{2} \operatorname{tr} \underline{\chi}+F \\
& F=\left(\operatorname{tr} \underline{\chi}_{0}\right)\left(\psi_{3} \nabla^{2} \psi_{3}+\left(\nabla \psi_{3}\right) \nabla \psi_{3}+\psi_{3} \psi_{3} \nabla \psi_{3}\right) \\
& +\psi_{3} \psi_{3} \nabla^{2} \psi_{3}+\psi_{3}\left(\nabla \psi_{3}\right) \nabla \psi_{3}+\psi_{3} \psi_{3} \psi_{3} \nabla \psi_{3} .
\end{aligned}
$$

Making use of our estimates for $\psi_{3}$, we easily derive, with a constant $C=C\left(\mathcal{O}^{(0))}, \mathcal{R}, \underline{\mathcal{R}}\right)$, that

$$
\|F\|_{\mathcal{L}_{(\mathrm{sc})}^{2}(\underline{H})} \lesssim \delta^{1 / 2} C
$$

Thus,

$$
\begin{aligned}
& \nabla_{3} \Delta \nabla \operatorname{tr} \underline{\chi}^{\prime}=\underline{\hat{\chi}} \Delta \nabla \underline{\hat{\chi}}+\underline{\chi} \nabla^{3} \operatorname{tr} \underline{\chi}+(\nabla \underline{\hat{\chi}}) \nabla^{2} \underline{\hat{\chi}}+(\nabla \underline{\chi}) \nabla^{2} \operatorname{tr} \underline{\chi}+F_{1}, \\
&\left\|F_{1}\right\|_{\mathcal{L}_{(\mathrm{sc})}^{2}(H)} \lesssim \delta^{1 / 2} C .
\end{aligned}
$$

Observe that neither the principal term $\underline{\hat{\chi}} \nabla \Delta \underline{\hat{\chi}}$ nor the lower-order term $(\nabla \underline{\hat{\chi}}) \nabla^{2} \underline{\hat{\chi}}$ appear to satisfy an $\mathcal{L}_{(\mathrm{sc})}^{2}(H)$ estimate. The principal terms seem particularly nasty, since we cannot possibly expect to estimate three derivatives of $\underline{\hat{\chi}}$ using norms which involve only one derivative of the curvature components. Clearly another renormalization is needed. In fact, we make use of equation (11.17), which we write in the form

$$
\Delta \nabla \underline{\widehat{\chi}}=\nabla_{3} \Delta \underline{\phi}-\left(\operatorname{tr} \underline{\chi}_{0}\right) \nabla^{2} \underline{\phi}-\underline{\widehat{\chi}} \nabla^{2} \underline{\phi}-E .
$$


We can thus replace the dangerous term $\Delta \nabla \underline{\hat{\chi}}$ in (11.26) and obtain

$$
\begin{aligned}
\nabla_{3} \Delta \nabla \operatorname{tr} \underline{\chi}^{\prime} & =\underline{\hat{\chi}} \nabla_{3} \Delta \underline{\phi}+\underline{\chi} \nabla^{3} \operatorname{tr} \underline{\chi}+(\nabla \underline{\hat{\chi}}) \nabla^{2} \underline{\hat{\chi}}+(\nabla \underline{\chi}) \nabla^{2} \operatorname{tr} \underline{\chi}+F_{2}, \\
F_{2} & =F_{1}-\left(\left(\operatorname{tr} \underline{\chi}_{0}\right) \nabla^{2} \underline{\phi}-\underline{\widehat{\chi}} \nabla^{2} \underline{\phi}-E\right) \underline{\hat{\chi}} .
\end{aligned}
$$

In view of our estimates for $\phi$, we have

$$
\left\|F_{2}\right\|_{\mathcal{L}_{(\mathrm{sc})}^{2}(\underline{H})} \lesssim C \delta^{1 / 2}\left(1+\delta^{1 / 2} C\right)\left\|\nabla^{2} \underline{\phi}\right\|_{\mathcal{L}_{(\mathrm{sc})}^{2}(\underline{H})} .
$$

Now, recalling also the definition of $\underline{\phi}$,

$$
\begin{aligned}
\nabla_{3}\left(\Delta \nabla \operatorname{tr} \underline{\chi^{\prime}}-\underline{\hat{\chi}} \Delta \underline{\phi}\right)=- & \left(\nabla_{3} \underline{\hat{\chi}}\right) \Delta \underline{\phi}+\left(\operatorname{tr} \underline{\chi}_{0}\right) \nabla^{3} \operatorname{tr} \underline{\chi}+\psi_{3} \nabla^{3} \operatorname{tr} \underline{\chi}+\left(\nabla_{3} \underline{\phi}\right) \nabla^{2} \underline{\chi} \\
& +(\nabla \operatorname{tr} \underline{\chi}) \nabla^{2} \operatorname{tr} \underline{\chi}+F_{2} .
\end{aligned}
$$

Hence,

$$
\begin{aligned}
& \left\|\Delta \nabla \operatorname{tr} \underline{\chi}^{\prime}\right\|_{\mathcal{L}_{(\mathrm{sc})}^{2}(u, \underline{u})} \lesssim\left\|\Delta \nabla \operatorname{tr} \underline{\chi}^{\prime}\right\|_{\mathcal{L}_{(\mathrm{sc})}^{2}(0, \underline{u})}+C \delta^{1 / 2}\|\underline{\hat{\chi}}\|_{\mathcal{L}_{(\mathrm{sc})}^{\infty}}\|\Delta \underline{\phi}\|_{\mathcal{L}_{(\mathrm{sc})}^{2}(u, \underline{u})} \\
& +\left(1+C \delta^{1 / 2}\right) \int_{0}^{u}\left\|\nabla^{3} \operatorname{tr} \underline{\chi}\right\|_{\mathcal{L}_{(\mathrm{sc})}^{2}\left(u^{\prime}, \underline{u}\right)} d u^{\prime} \\
& +C \delta^{1 / 2}\left\|\nabla_{3} \underline{\hat{\chi}}\right\|_{\operatorname{Tr}_{(\mathrm{sc})}(\underline{H})}\|\Delta \underline{\phi}\|_{\mathcal{L}_{(\mathrm{sc})}^{2}(\underline{H})} \\
& +\delta^{1 / 2}\left\|\nabla_{3} \underline{\phi}\right\|_{\operatorname{Tr}_{(\mathrm{sc})}(\underline{H})}\left\|\nabla^{2} \underline{\chi}\right\|_{\mathcal{L}_{(\mathrm{sc})}^{2}(\underline{H})} \\
& +\delta^{1 / 2}\|\nabla \operatorname{tr} \underline{\chi}\|_{\mathcal{L}_{(\mathrm{sc})}^{\infty}}\left\|\nabla^{2} \operatorname{tr} \underline{\chi}\right\|_{\mathcal{L}_{(\mathrm{sc})}^{2}(\underline{H})} \\
& +\left\|F_{2}\right\|_{\mathcal{L}_{(\mathrm{sc})}^{2}(\underline{H})} .
\end{aligned}
$$

Using the calculus inequalities of $\S 4.4$ and our estimates for $\nabla^{2} \nabla_{3} \operatorname{tr} \underline{\chi}$,

$$
\|\nabla \operatorname{tr} \underline{\chi}\|_{\mathcal{L}_{(\mathrm{sc})}^{\infty}} \lesssim C+\left\|\nabla^{3} \operatorname{tr} \underline{\chi}\right\|_{\mathcal{L}_{(\mathrm{sc})}(\underline{H})}
$$

Also, in view of the trace estimate (11.14),

$$
\left\|\nabla_{3} \underline{\phi}\right\|_{\operatorname{Tr}_{(\mathrm{sc})}(\underline{H})} \lesssim C+\left\|\nabla^{3} \operatorname{tr} \underline{\chi}\right\|_{\mathcal{L}_{(\mathrm{sc})}(\underline{H})} .
$$

Hence,

$$
\begin{aligned}
\left\|\Delta \nabla \operatorname{tr} \underline{\chi}^{\prime}\right\|_{\mathcal{L}_{(\mathrm{sc})}^{2}(u, \underline{u})} \lesssim \| & \Delta \nabla \operatorname{tr} \underline{\chi}^{\prime}\left\|_{\mathcal{L}_{(\mathrm{sc})}^{2}(0, \underline{u})}+C \delta^{1 / 2}\right\| \nabla^{2} \underline{\phi} \|_{\mathcal{L}_{(\mathrm{sc})}^{2}(u, \underline{u})} \\
& +\left(1+C \delta^{1 / 2}\right) \int_{0}^{u}\left\|\nabla^{3} \operatorname{tr} \underline{\chi}\right\|_{\mathcal{L}_{(\mathrm{sc})}^{2}\left(u^{\prime}, \underline{u}\right)} d u^{\prime} \\
& +C \delta^{1 / 2}\left\|\nabla_{3} \underline{\hat{\chi}}\right\|_{\operatorname{Tr}(\mathrm{sc})(\underline{H})}\|\Delta \underline{\phi}\|_{\mathcal{L}_{(\mathrm{sc})}^{2}(\underline{H})} \\
& +C \delta^{1 / 2}\left\|\nabla^{3} \operatorname{tr} \underline{\underline{\chi}}\right\|_{\mathcal{L}_{(\mathrm{sc})}^{2}(\underline{H})}+C^{2} \delta^{1 / 2}
\end{aligned}
$$


Now,

$$
\left\|\Delta \nabla \operatorname{tr} \underline{\chi}^{\prime}\right\|_{\mathcal{L}_{(\mathrm{sc})}^{2}(u, \underline{u})} \lesssim\|\Delta \nabla \operatorname{tr} \underline{\chi}\|_{\mathcal{L}_{(\mathrm{sc})}^{2}(u, \underline{u})}+\delta^{1 / 2} C\left(\left\|\nabla^{2} \underline{\omega}\right\|_{\mathcal{L}_{(\mathrm{sc})}^{2}(u, \underline{u})}+C \delta^{1 / 2}\right) .
$$

Using the elliptic estimates discussed in $§ 4.6$ and our estimates for $K$, we deduce that

$$
\begin{aligned}
& \left\|\nabla^{3} \operatorname{tr} \underline{\chi}\right\|_{\mathcal{L}_{\text {(sc) }}^{2}(S)} \lesssim\|\Delta \nabla \operatorname{tr} \underline{\chi}\|_{\mathcal{L}_{\text {(sc) }}^{2}(S)} \\
& +\delta^{1 / 2}\left(\|\nabla K\|_{\mathcal{L}_{\text {(sc) }}^{2}(S)}\|\nabla \operatorname{tr} \underline{\chi}\|_{\mathcal{L}_{\text {(sc) }}^{\infty}(S)}+\|K\|_{\mathcal{L}_{(\mathrm{sc})}^{4}(S)}\left\|\nabla^{2} \operatorname{tr} \underline{\chi}\right\|_{\mathcal{L}_{(\mathrm{sc})}^{4}(S)}\right) \\
& \lesssim\|\Delta \nabla \operatorname{tr} \underline{\chi}\|_{\mathcal{L}_{\text {(sc) }}^{2}(S)}+\delta^{1 / 2}\left(\|\nabla \operatorname{tr} \underline{\chi}\|_{\mathcal{L}_{\text {(sc) }}^{\infty}(S)}+\left\|\nabla^{2} \operatorname{tr} \underline{\chi}\right\|_{\mathcal{L}_{\text {(sc) }}^{4}(S)}\right) \\
& \lesssim\|\Delta \nabla \operatorname{tr} \underline{\chi}\|_{\mathcal{L}_{(\mathrm{sc})}^{2}(S)}+\delta^{1 / 2}\left(C+\left\|\nabla^{3} \operatorname{tr} \underline{\chi}\right\|_{\mathcal{L}_{(\mathrm{sc})}^{2}(\underline{H})}\right) \text {. }
\end{aligned}
$$

Hence, after using Grönwall's inequality,

$$
\begin{aligned}
\left\|\nabla^{3} \operatorname{tr} \underline{\chi}\right\|_{\mathcal{L}_{\text {(sc) }}^{2}(u, \underline{u})} \lesssim & \left\|\nabla^{3} \operatorname{tr} \underline{\chi}\right\|_{\mathcal{L}_{(\mathrm{sc})}^{2}(0, \underline{u})}+C \delta^{1 / 2}\left(\left\|\nabla^{2} \underline{\omega}\right\|_{\mathcal{L}_{\text {(sc) }}^{2}(u, \underline{u})}+\left\|\nabla^{2} \underline{\phi}\right\|_{\mathcal{L}_{(\mathrm{sc})}^{2}(u, \underline{u})}\right) \\
& +C \delta^{1 / 2}\left\|\nabla_{3} \underline{\hat{\chi}}\right\|_{\operatorname{Tr}_{(\mathrm{sc})}(\underline{H})}\|\Delta \underline{\phi}\|_{\mathcal{L}_{\text {(sc) }}^{2}(\underline{H})} \\
& +C \delta^{1 / 2}\left\|\nabla^{3} \operatorname{tr} \underline{\chi}\right\|_{\mathcal{L}_{(\mathrm{sc})}^{2}(\underline{H})}+C^{2} \delta^{1 / 2}
\end{aligned}
$$

Thus, after integration,

$$
\left\|\nabla^{3} \operatorname{tr} \underline{\chi}\right\|_{\mathcal{L}_{(\mathrm{sc})}^{2}\left(\underline{H}^{(0, u)}\right)}^{2} \lesssim C^{2}+C^{2} \delta \int_{0}^{u}\left\|\nabla_{3} \underline{\widehat{\chi}}_{\operatorname{Tr}_{(\mathrm{sc})}\left(\underline{H}^{\left(0, u^{\prime}\right)}\right)}^{2}\right\| \Delta \underline{\phi} \|_{\mathcal{L}_{\text {(sc) }}^{2}\left(\underline{H}^{\left(0, u^{\prime}\right)}\right)}^{2} d u^{\prime} .
$$

It remains to estimate the trace norm $\left\|\nabla_{3} \underline{\widehat{\chi}}\right\|_{\operatorname{Tr}_{(\mathrm{sc})}\left(\underline{H}^{\left(0, u^{\prime}\right)}\right)}$. We claim the following.

Lemma 11.4. There exists a constant $C$ depending only on $\mathcal{O}^{(0)}, \mathcal{R}, \underline{\mathcal{R}}$ as well as $\left\|\nabla_{3} \underline{\alpha}\right\|_{\mathcal{L}_{(\mathrm{sc})}^{2}(\underline{H})}$ such that

$$
\left\|\nabla_{3} \underline{\hat{\chi}}\right\|_{\operatorname{Tr}_{(\mathrm{sc})}(\underline{H})} \lesssim C \delta^{-1 / 2} .
$$

Proof. In view of the trace estimate (11.2), for $\underline{H}=\underline{H}^{\left(0, u^{\prime}\right)}$ we have

$$
\begin{aligned}
& \left\|\nabla_{3} \underline{\hat{\chi}}\right\|_{\operatorname{Tr}_{(\mathrm{sc})}(\underline{H})} \lesssim\left\|\nabla_{3}^{2} \underline{\hat{\chi}}\right\|_{\mathcal{L}_{\text {(sc) }}^{2}(\underline{H})}+\left\|\nabla \nabla_{3} \underline{\hat{\chi}}\right\|_{\mathcal{L}_{\text {(sc) }}^{2}(\underline{H})} \\
& +\left\|\nabla^{2} \underline{\hat{\chi}}\right\|_{\mathcal{L}_{(\mathrm{sc})}^{2}(\underline{H})}+\|\underline{\hat{\chi}}\|_{\mathcal{L}_{(\mathrm{sc})}^{2}(\underline{H})}+\left(1+C \delta^{1 / 2}\right)\|\underline{\hat{\chi}}\|_{\mathcal{L}_{(\mathrm{sc})}^{\infty}} .
\end{aligned}
$$

Observe that

$$
\left\|\nabla_{3} \underline{\widehat{\chi}}\right\|_{\mathcal{L}_{(\mathrm{sc})}^{2}(\underline{H})}+\|\underline{\hat{\chi}}\|_{\mathcal{L}_{(\mathrm{sc})}^{2}(\underline{H})} \lesssim C \delta^{-1 / 2} .
$$

We also claim that

$$
\left\|\nabla_{3}^{2} \underline{\widehat{\chi}}\right\|_{\mathcal{L}_{(\mathrm{sc})}^{2}(\underline{H})} \lesssim C \delta^{-1 / 2}+\left\|\nabla_{3} \underline{\alpha}\right\|_{\mathcal{L}_{(\mathrm{sc})}^{2}(\underline{H})} .
$$


Indeed, differentiating,

$$
\nabla_{3} \underline{\hat{\chi}}=-\underline{\alpha}-(\operatorname{tr} \underline{\chi}) \underline{\hat{\chi}}-2 \underline{\omega} \underline{\hat{\chi}}
$$

Thus,

$$
\nabla_{3}^{2} \underline{\hat{\chi}}=-\nabla_{3} \underline{\alpha}-\left(\nabla_{3} \operatorname{tr} \underline{\chi}\right) \underline{\hat{\chi}}-(\operatorname{tr} \underline{\chi}) \nabla_{3} \underline{\widehat{\chi}}-2\left(\nabla_{3} \underline{\omega}\right) \underline{\widehat{\chi}}-\underline{\omega} \nabla_{3} \underline{\hat{\chi}} .
$$

Hence,

$$
\begin{aligned}
&\left\|\nabla_{3}^{2} \underline{\hat{\chi}}\right\|_{\mathcal{L}_{(\mathrm{sc})}^{2}(\underline{H})} \lesssim\left\|\nabla_{3} \underline{\alpha}\right\|_{\mathcal{L}_{(\mathrm{sc})}^{2}(\underline{H})}+\|\underline{\widehat{\chi}}\|_{\mathcal{L}_{(\mathrm{sc})}^{2}(\underline{H})}+C \delta^{1 / 2}\left(\left\|\nabla_{3} \underline{\omega}\right\|_{\mathcal{L}_{(\mathrm{sc})}^{2}(\underline{H})}+\| \nabla_{3} \underline{\widehat{\chi}}_{\mathcal{L}_{(\mathrm{sc})}^{2}(\underline{H})}\right) \\
& \lesssim C \delta^{-1 / 2}+\left\|\nabla_{3} \underline{\alpha}\right\|_{\mathcal{L}_{(\mathrm{sc})}^{2}(\underline{H})}
\end{aligned}
$$

which completes the proof of our estimate.

Returning to (11.27), we have, with a constant $C$ depending on $\mathcal{O}^{(0)}, \mathcal{R}, \underline{\mathcal{R}}$ as well as $\left\|\nabla_{3} \underline{\alpha}\right\|_{\mathcal{L}_{(\mathrm{sc})}^{2}(\underline{H})}$,

$$
\begin{aligned}
\left\|\nabla^{3} \operatorname{tr} \underline{\chi}\right\|_{\mathcal{L}_{(\mathrm{sc})}^{2}\left(\underline{H}^{(0, u)}\right)}^{2} & \lesssim C^{2}+C^{2} \int_{0}^{u}\left\|\nabla^{2} \underline{\phi}\right\|_{\mathcal{L}_{(\mathrm{sc})}^{2}\left(\underline{H}^{\left(0, u^{\prime}\right)}\right)}^{2} d u^{\prime} \\
& \lesssim C^{2}\left(1+\int_{0}^{u}\left\|\nabla^{3} \operatorname{tr} \underline{\chi}\right\|_{\mathcal{L}_{(\mathrm{sc})}^{2}\left(\underline{H}^{\left(0, u^{\prime}\right)}\right)}^{2} d u^{\prime}\right) .
\end{aligned}
$$

Thus, applying Grönwall's inequality once more, we derive that

$$
\left\|\nabla^{3} \operatorname{tr} \underline{\chi}\right\|_{\mathcal{L}_{(\mathrm{sc})}^{2}\left(\underline{H}^{(0, u)}\right)}^{2} \lesssim C^{2} .
$$

This finishes the proof of the second part of the following proposition.

Proposition 11.5. The following estimates hold true with a constant $C$ depending on $\mathcal{O}^{(0)}, \mathcal{R}, \underline{\mathcal{R}}$ as well as $\sup _{u}\left\|\nabla_{4} \alpha\right\|_{\mathcal{L}_{(\mathrm{sc})}^{2}\left(H_{u}\right)}$ and $\sup _{\underline{u}}\left\|\nabla_{3} \underline{\alpha}\right\|_{\mathcal{L}_{(\mathrm{sc})}^{2}\left(\underline{H}_{\underline{u}}\right)}$.

(1) We have, along $H=H_{u}$,

$$
\begin{gathered}
\left\|\nabla^{3} \operatorname{tr} \chi\right\|_{\mathcal{L}_{(\mathrm{sc})}^{2}(H)}+\|\nabla \operatorname{tr} \chi\|_{\mathcal{L}_{(\mathrm{sc})}^{\infty}} \lesssim C, \\
\sup _{S}\|\nabla \hat{\chi}\|_{\mathcal{L}_{\text {(sc) }}^{4}(S)}+\|\nabla \widehat{\chi}\|_{\operatorname{Tr}_{(\mathrm{sc})}(H)} \lesssim C .
\end{gathered}
$$

(2) We have, along $\underline{H}=\underline{H}_{\underline{u}}$,

$$
\begin{gathered}
\left\|\nabla^{3} \operatorname{tr} \underline{\chi}\right\|_{\mathcal{L}_{(\mathrm{sc})}^{2}(\underline{H})}+\|\nabla \operatorname{tr} \underline{\chi}\|_{\mathcal{L}_{(\mathrm{sc})}^{\infty}} \lesssim C, \\
\sup _{S}\|\nabla \underline{\hat{\chi}}\|_{\mathcal{L}_{(\mathrm{sc})}^{4}(S)}+\|\nabla \underline{\hat{\chi}}\|_{\operatorname{Tr}_{(\mathrm{sc})}(\underline{H})} \lesssim C .
\end{gathered}
$$




\subsection{Estimates for the trace norms of $\nabla \eta$ and $\nabla \underline{\eta}$}

As in the previous subsection, we need a series of renormalizations. The proof follows, however, the same outline as above. We first prove the following result.

Proposition 11.6. Consider the following transport equations along $H=H_{u}$, resp. $\underline{H}=\underline{H}_{\underline{u}}$,

$$
\begin{array}{rlrl}
\nabla_{4}{ }^{(4)} \phi & =\nabla \eta, & { }^{(4)} \phi(0, \underline{u})=0, \\
\nabla_{4}{ }^{(4)} \underline{\phi}=\nabla \underline{\eta}, & { }^{(4)} \underline{\phi}(0, \underline{u})=0,
\end{array}
$$

and

$$
\begin{array}{rlrl}
\nabla_{3}{ }^{(3)} \phi & =\nabla \eta, & { }^{(3)} \phi(0, \underline{u})=0, \\
\nabla_{3}{ }^{(3)} \underline{\phi}=\nabla \underline{\eta}, & { }^{(3)} \underline{\phi}(0, \underline{u})=0 .
\end{array}
$$

(1) Solutions $\phi=\left({ }^{(4)} \phi,{ }^{(4)} \phi\right)$ of (11.28) and (11.29) satisfy the estimates

$$
\begin{aligned}
&\|\phi\|_{\mathcal{L}_{\text {(sc) }}^{2}(S)}+\|\phi\|_{\mathcal{L}_{(\mathrm{sc})}^{4}(S)}+\|\nabla \phi\|_{\mathcal{L}_{(\mathrm{sc})}^{2}(S)}+\left\|\nabla_{4} \phi\right\|_{\mathcal{L}_{\text {(sc) }}^{2}(S)} \lesssim C, \\
&\left\|\nabla \nabla_{4} \phi\right\|_{\mathcal{L}_{(\mathrm{sc})}^{2}(H)}+\left\|\nabla_{4}^{2} \phi\right\|_{\mathcal{L}_{\text {(sc) }}^{2}(H)} \lesssim C,
\end{aligned}
$$

with a constant $C=C\left(\mathcal{O}^{(0)}, \mathcal{R}, \underline{\mathcal{R}}\right)$. Moreover,

$$
\left\|\nabla^{2} \phi\right\|_{\mathcal{L}_{(\mathrm{sc})}^{2}(H)} \lesssim\left\|\nabla^{2} \mu\right\|_{\mathcal{L}_{(\mathrm{sc})}^{2}(H)}+C .
$$

As a consequence (see the calculus inequalities of $\S 4.4$ ), we also have

$$
\|\phi\|_{\mathcal{L}_{(\text {(sc) }}^{\infty}} \lesssim\left\|\nabla^{2} \mu\right\|_{\mathcal{L}_{\text {(sc) }}^{2}(H)}+C
$$

and as a consequence of the trace estimate (11.1),

$$
\left\|\nabla_{4} \phi\right\|_{\operatorname{Tr}_{(\mathrm{sc})}(H)} \lesssim\left\|\nabla^{2} \mu\right\|_{\mathcal{L}_{\text {(sc) }}^{2}(H)}+C .
$$

(2) Solutions $\underline{\phi}=\left({ }^{(3)} \phi,{ }^{(3)} \underline{\phi}\right)$ of (11.30) and (11.31) satisfy the estimates

$$
\begin{array}{r}
\|\underline{\phi}\|_{\mathcal{L}_{(\mathrm{sc})}^{2}(S)}+\|\underline{\phi}\|_{\mathcal{L}_{(\mathrm{sc})}^{4}(S)}+\|\nabla \underline{\phi}\|_{\mathcal{L}_{(\mathrm{sc})}^{2}(S)}+\left\|\nabla_{3} \underline{\phi}\right\|_{\mathcal{L}_{(\mathrm{sc})}^{2}(S)} \lesssim C, \\
\left\|\nabla \nabla_{3} \underline{\phi}\right\|_{\mathcal{L}_{(\mathrm{sc})}^{2}(\underline{H})}+\left\|\nabla_{3}^{2} \phi\right\|_{\mathcal{L}_{(\mathrm{sc})}^{2}(\underline{H})} \lesssim C,
\end{array}
$$

with a constant $C=C\left(\mathcal{O}^{(0)}, \mathcal{R}, \underline{\mathcal{R}}\right)$. Moreover,

$$
\left\|\nabla^{2}\left({ }^{(3)} \phi,{ }^{(3)} \underline{\phi}\right)\right\|_{\mathcal{L}_{(\mathrm{sc})}^{2}(\underline{H})} \lesssim\left\|\nabla^{2} \underline{\mu}\right\|_{\mathcal{L}_{\text {(sc) }}^{2}(\underline{H})}+C .
$$

As a consequence (see the calculus inequalities of $\S 4.4$ ), we also have

$$
\left\|\left({ }^{(3)} \phi,{ }^{(3)} \underline{\phi}\right)\right\|_{\mathcal{L}_{(\mathrm{sc})}^{\infty}} \lesssim\left\|\nabla^{2} \underline{\mu}\right\|_{\mathcal{L}_{(\mathrm{sc})}^{2}(\underline{H})}+C,
$$

and as a consequence of the trace estimate (11.2),

$$
\left\|\nabla_{3}\left({ }^{(3)} \phi,{ }^{(3)} \underline{\phi}\right)\right\|_{\operatorname{Tr}_{(\mathrm{sc})}(\underline{H})} \lesssim\left\|\nabla^{2} \underline{\mu}\right\|_{\mathcal{L}_{(\mathrm{sc})}^{2}(\underline{H})}+C .
$$


Proof. We start with

$$
\nabla_{3}^{(3)} \phi=\nabla \eta \quad \text { and } \quad \nabla_{3}^{(3)} \underline{\phi}=\nabla \underline{\eta} .
$$

Commuting both equations with $\Delta$, and proceeding exactly as in the derivation of (11.18), we get

$$
\begin{aligned}
\nabla_{3} \Delta^{(3)} \phi & =\nabla \Delta \eta+\left(\operatorname{tr} \underline{\chi}_{0}\right) \nabla^{2(3)} \phi+\underline{\widehat{\chi}} \nabla^{2(3)} \phi+E, \\
\nabla_{3} \Delta^{(3)} \underline{\phi} & =\nabla \Delta \underline{\eta}+\left(\operatorname{tr} \underline{\chi}_{0}\right) \nabla^{2(3)} \underline{\phi}+\underline{\widehat{\chi}} \nabla^{2(3)} \underline{\phi}+\underline{E}, \\
\|E\|_{\mathcal{L}_{(\mathrm{sc})}^{2}(\underline{H})} & \lesssim C \delta^{1 / 2}\left(C+\left\|\nabla^{2(3)} \phi\right\|_{\mathcal{L}_{(\mathrm{sc})}^{2}(\underline{H})}\right), \\
\|\underline{E}\|_{\mathcal{L}_{\text {(sc) }}^{2}(\underline{H})} & \lesssim C \delta^{1 / 2}\left(C+\left\|\nabla^{2(3)} \underline{\phi}\right\|_{\mathcal{L}_{(\mathrm{sc})}^{2}(\underline{H})}\right) .
\end{aligned}
$$

Recall, see (6.12) and (6.13), that

$$
\begin{array}{ll}
\operatorname{div} \eta=-\mu-\varrho, & \operatorname{curl} \eta=\sigma-\frac{1}{2} \widehat{\chi} \wedge \underline{\hat{\chi}}, \\
\operatorname{div} \underline{\eta}=-\underline{\mu}-\varrho, & \operatorname{curl} \underline{\eta}=-\sigma+\frac{1}{2} \widehat{\chi} \wedge \underline{\widehat{\chi}},
\end{array}
$$

i.e., schematically,

$$
\begin{aligned}
& { }^{\star} \mathcal{D}_{1} \mathcal{D}_{1} \eta={ }^{\star} \mathcal{D}_{1}(-\mu-\varrho, \sigma-\widehat{\chi} \wedge \underline{\hat{\chi}}), \\
& { }^{\star} \mathcal{D}_{1} \mathcal{D}_{1} \underline{\eta}={ }^{\star} \mathcal{D}_{1}(-\underline{\mu}-\varrho, \sigma-\widehat{\chi} \wedge \underline{\hat{\chi}}) .
\end{aligned}
$$

Proceeding as in the derivation of (11.19) we find, schematically,

$$
\begin{aligned}
\nabla \Delta \eta & =\nabla^{2} \mu+\nabla^{2}(\varrho, \sigma)+F_{1}, \\
\nabla \Delta \underline{\eta} & =\nabla^{2} \underline{\mu}+\nabla^{2}(\varrho, \sigma)+F_{1}, \\
\left\|F_{1}\right\|_{\mathcal{L}_{(\mathrm{sc})}^{2}(\underline{H})} & \lesssim C .
\end{aligned}
$$

We now make use, see (6.14) and (6.16), of the equations

$$
\begin{aligned}
\nabla_{3} \omega & =\frac{1}{2} \varrho+2 \omega \underline{\omega}+\frac{3}{4}|\eta-\underline{\eta}|^{2}+\frac{1}{4}(\eta-\underline{\eta})(\eta+\underline{\eta})-\frac{1}{8}|\eta+\underline{\eta}|^{2} \\
\nabla_{3} \omega^{\dagger} & =\frac{1}{2} \sigma .
\end{aligned}
$$

Proceeding now exactly as in the derivation of (11.20) and (11.21), we deduce that

$$
\begin{aligned}
\nabla^{2}(\varrho, \sigma) & =\nabla_{3} \nabla^{2}\left(\omega, \omega^{\dagger}\right)+F_{2}, \\
\left\|F_{2}\right\|_{\mathcal{L}_{(\mathrm{sc})}^{2}(\underline{H})} & \lesssim C .
\end{aligned}
$$


Therefore, just as before for the derivation of $\nabla \Delta \hat{\chi}$, schematically,

$$
\begin{aligned}
\nabla \Delta \eta & =\nabla_{3} \nabla^{2}\left(\omega, \omega^{\dagger}\right)+\nabla^{2} \mu+F, \\
\nabla \Delta \underline{\eta} & =\nabla_{3} \nabla^{2}\left(\omega, \omega^{\dagger}\right)+\nabla^{2} \underline{\mu}+\underline{F} \\
\|(F, \underline{F})\|_{\mathcal{L}_{(\mathrm{sc})}(\underline{H})} & \lesssim C .
\end{aligned}
$$

Thus, back to (11.39) and (11.40), we deduce (just as in (11.23)) that

$$
\begin{aligned}
& \nabla_{3}\left(\Delta^{(3)} \phi-\nabla^{2}\left(\omega, \omega^{\dagger}\right)\right)=\nabla^{2} \mu+\left(\operatorname{tr} \underline{\chi}_{0}\right) \nabla^{2(3)} \phi+\underline{\widehat{\chi}} \nabla^{2(3)} \phi+E, \\
&\|E\|_{\mathcal{L}_{(\mathrm{sc})}^{2}(\underline{H})} \lesssim C\left(1+\delta^{1 / 2}\left\|\nabla^{2(3)} \phi\right\|_{\mathcal{L}_{(\mathrm{sc})}^{2}(\underline{H})}\right)
\end{aligned}
$$

and

$$
\begin{aligned}
& \nabla_{3}\left(\Delta^{(3)} \underline{\phi}-\nabla^{2}\left(\omega, \omega^{\dagger}\right)\right)=\nabla^{2} \underline{\mu}+\left(\operatorname{tr} \underline{\chi}_{0}\right) \nabla^{2(3)} \underline{\phi}+\underline{\widehat{\chi}} \nabla^{2(3)} \underline{\phi}+\underline{E}, \\
&\|\underline{E}\|_{\mathcal{L}_{(\mathrm{sc})}^{2}(\underline{H})} \lesssim C\left(1+\delta^{1 / 2}\left\|\nabla^{2(3)} \underline{\phi}\right\|_{\mathcal{L}_{(\mathrm{sc})}^{2}(\underline{H})}\right) .
\end{aligned}
$$

We then proceed with elliptic $\mathcal{L}_{(\mathrm{sc})}^{2}$ estimates, exactly as in (11.24) and, after using also Grönwall's inequality, we find (as in (11.25)) that

$$
\begin{gathered}
\left\|\nabla^{2(3)} \phi\right\|_{\mathcal{L}_{(\mathrm{sc})}^{2}(u, \underline{u})} \lesssim\left\|\nabla^{2}\left(\omega, \omega^{\dagger}\right)\right\|_{\mathcal{L}_{(\mathrm{sc})}^{2}(u, \underline{u})}+\int_{0}^{u}\left\|\nabla^{2} \mu\right\|_{\mathcal{L}_{(\mathrm{sc})}^{2}\left(u^{\prime}, \underline{u}\right)} d u^{\prime} \\
+C\left(1+\delta^{1 / 2}\right)\left\|\nabla^{2(3)} \phi\right\|_{\mathcal{L}_{(\mathrm{sc})}^{2}(\underline{H})}
\end{gathered}
$$

and

$$
\begin{aligned}
\left\|\nabla^{2(3)} \underline{\phi}\right\|_{\mathcal{L}_{(\mathrm{sc})}^{2}(u, \underline{u})} \lesssim & \left\|\nabla^{2}\left(\omega, \omega^{\dagger}\right)\right\|_{\mathcal{L}_{(\mathrm{sc})}^{2}(u, \underline{u})}+\int_{0}^{u}\left\|\nabla^{2} \underline{\mu}\right\|_{\mathcal{L}_{(\mathrm{sc})}^{2}\left(u^{\prime}, \underline{u}\right)} d u^{\prime} \\
& +C\left(1+\delta^{1 / 2}\right)\left\|\nabla^{2(3)} \underline{\phi}\right\|_{\mathcal{L}_{(\mathrm{sc})}^{2}(\underline{H})} .
\end{aligned}
$$

Integrating, we deduce, for $C \delta^{1 / 2}$ sufficiently small, that

$$
\begin{aligned}
&\left\|\nabla^{2(3)} \phi\right\|_{\mathcal{L}_{(\mathrm{sc})}^{2}(\underline{H})} \lesssim C+\left\|\nabla^{2} \mu\right\|_{\mathcal{L}_{(\mathrm{sc})}^{2}(\underline{H})}, \\
&\left\|\nabla^{2(3)} \underline{\phi}\right\|_{\mathcal{L}_{(\mathrm{sc})}^{2}(\underline{H})} \lesssim C+\left\|\nabla^{2} \underline{\mu}\right\|_{\mathcal{L}_{(\mathrm{sc})}^{2}(\underline{H})},
\end{aligned}
$$

as desired.

It remains to estimate $\left\|\nabla^{2} \mu\right\|_{\mathcal{L}_{(\mathrm{sc})}^{2}(H)}$ and $\left\|\nabla^{2} \underline{\mu}\right\|_{\mathcal{L}_{(\mathrm{sc})}^{2}(\underline{H})}$. As before, we treat only the estimate for the slightly more difficult case of $\underline{\mu}$. In view of the proof of the previous proposition, we have (neglecting signs and constants, as before)

$$
\begin{aligned}
& \nabla \Delta \eta=\nabla_{3} \Delta^{(3)} \phi+\left(\operatorname{tr} \underline{\chi}_{0}\right) \nabla^{2(3)} \phi+\underline{\widehat{\chi}} \nabla^{2(3)} \phi+E, \\
& \nabla \Delta \underline{\eta}=\nabla_{3} \Delta^{(3)} \underline{\phi}+\left(\operatorname{tr} \underline{\chi}_{0}\right) \nabla^{2(3)} \underline{\phi}+\underline{\widehat{\chi}} \nabla^{2(3)} \underline{\phi}+\underline{E}, \\
&\|E\|_{\mathcal{L}_{(\mathrm{sc})}^{2}(\underline{H})} \lesssim C \delta^{1 / 2}\left(C+\left\|\nabla^{2(3)} \phi\right\|_{\mathcal{L}_{(\mathrm{sc})}^{2}(\underline{H})}\right) \\
&\|\underline{E}\|_{\mathcal{L}_{(\mathrm{sc})}^{2}(\underline{H})} \lesssim C \delta^{1 / 2}\left(C+\left\|\nabla^{2(3)} \underline{\phi}\right\|_{\mathcal{L}_{(\mathrm{sc})}^{2}(\underline{H})}\right) .
\end{aligned}
$$


We start with the transport equation (6.9),

$$
\begin{aligned}
\nabla_{3} \underline{\mu}+(\operatorname{tr} \underline{\chi}) \underline{\mu}=- & \frac{1}{2}(\operatorname{tr} \underline{\chi}) \operatorname{div} \eta+(\underline{\eta}-\eta) \nabla \operatorname{tr} \underline{\chi}+\underline{\hat{\chi}} \nabla(2 \underline{\eta}-\eta)+\frac{1}{2} \hat{\chi} \underline{\alpha}-(\underline{\eta}-3 \eta) \underline{\beta} \\
& +\frac{1}{2}(\operatorname{tr} \underline{\chi}) \varrho+\frac{1}{2}(\operatorname{tr} \underline{\chi})\left(|\underline{\eta}|^{2}-\underline{\eta} \eta\right)+\frac{1}{2}(\eta+\underline{\eta}) \underline{\hat{\chi}}(\underline{\eta}-\eta) .
\end{aligned}
$$

Commuting with the laplacian, we derive

$$
\begin{aligned}
\nabla_{3} \Delta \underline{\mu}=\underline{\hat{\chi}} \Delta \nabla(\underline{\eta}+\eta)+(\operatorname{tr} \underline{\chi}) \Delta \operatorname{div} \eta+(\nabla \eta+\nabla \underline{\eta}) \nabla^{2} \underline{\hat{\chi}}+(\operatorname{tr} \underline{\chi}) \Delta \underline{\mu} \\
+\left(\nabla^{2} \eta+\nabla^{2} \underline{\eta}\right) \nabla \underline{\hat{\chi}}+\frac{1}{2} \hat{\chi} \Delta \underline{\alpha}-(\underline{\eta}-3 \eta) \Delta \underline{\beta}+\frac{1}{2}(\operatorname{tr} \underline{\chi}) \Delta \varrho+\operatorname{Err} .
\end{aligned}
$$

Here, and in what follows, Err denotes any term which allows a bound of the form

$$
\|\operatorname{Err}\|_{\mathcal{L}_{(\mathrm{sc})}^{2}(\underline{H})} \lesssim C .
$$

Using the equation $\nabla_{3} \underline{\hat{\chi}}=-\underline{\alpha}-\left(\operatorname{tr} \underline{\chi}_{0}\right) \underline{\hat{\chi}}+\psi_{3} \psi_{3}$, we write

$$
\Delta \underline{\alpha}=-\nabla_{3} \Delta \underline{\hat{\chi}}+\text { Err. }
$$

Using the equation $\nabla_{3} \underline{\eta}=\underline{\beta}+\underline{\chi}(\eta-\underline{\eta})$, we can write

$$
\not \Delta \underline{\beta}=\nabla_{3} \Delta \underline{\eta}+\text { Err. }
$$

Using the equation $\nabla_{3} \omega=\frac{1}{2} \varrho+\psi \psi$, we can write

$$
\Delta \varrho=2 \nabla_{3} \Delta \omega+\text { Err. }
$$

Therefore, we can write

$$
\begin{gathered}
\nabla_{3} \Delta \underline{\mu}=\underline{\widehat{\chi}} \nabla_{3} \Delta\left({ }^{(3)} \phi+{ }^{(3)} \underline{\phi}\right)+(\operatorname{tr} \underline{\chi}) \nabla_{3} \Delta\left({ }^{(3)} \phi+{ }^{(3)} \underline{\phi}\right)+\left(\nabla_{3}\left({ }^{(3)} \phi+{ }^{(3)} \phi\right)\right) \nabla^{2} \underline{\widehat{\chi}} \\
+\left(\nabla^{2}(\eta+\underline{\eta})\right) \nabla \underline{\hat{\chi}}+(\operatorname{tr} \underline{\chi}) \nabla_{3} \Delta \omega+(\eta+\underline{\eta}) \nabla_{3} \Delta \underline{\eta}+\underline{\widehat{\chi}} \nabla_{3} \Delta \underline{\hat{\chi}}+\operatorname{Err}_{\phi},
\end{gathered}
$$

with $\operatorname{Err}_{\phi}$ verifying

$$
\left\|\operatorname{Err}_{\phi}\right\|_{\mathcal{L}_{(\mathrm{sc})}^{2}(\underline{H})} \lesssim C\left(1+\left\|\nabla^{2}\left({ }^{(3)} \phi+{ }^{(3)} \underline{\phi}\right)\right\|_{\mathcal{L}_{(\mathrm{sc})}^{2}(\underline{H})}\right) \lesssim C\left(1+\left\|\nabla^{2} \underline{\mu}\right\|_{\mathcal{L}_{(\mathrm{sc})}^{2}(\underline{H})}\right) .
$$

Therefore, introducing the renormalized quantity

$$
\underline{\mu}=\Delta \underline{\mu}-\underline{\chi} \Delta\left({ }^{(3)} \phi+{ }^{(3)} \underline{\phi}\right)-(\operatorname{tr} \underline{\chi}) \Delta \omega-(\eta+\underline{\eta}) \Delta \underline{\eta}-\underline{\hat{\chi}} \Delta \underline{\hat{\chi}},
$$

we have

$$
\begin{aligned}
\nabla_{3} \underline{\mu}=- & \left(\nabla_{3} \underline{\chi}\right) \Delta\left({ }^{(3)} \phi+{ }^{(3)} \underline{\phi}+\underline{\hat{\chi}}\right)-\left(\nabla_{3} \operatorname{tr} \underline{\chi}\right) \Delta\left({ }^{(3)} \phi+{ }^{(3)} \underline{\phi}\right)+\left(\nabla_{3}\left({ }^{(3)} \phi+{ }^{(3)} \underline{\phi}\right)\right) \nabla^{2} \underline{\widehat{\chi}} \\
& +\left(\nabla^{2}(\eta+\underline{\eta})\right) \nabla \underline{\hat{\chi}}+\left(\nabla_{3} \operatorname{tr} \underline{\chi}\right) \Delta \omega+\left(\nabla_{3}(\eta+\underline{\eta})\right) \Delta \underline{\eta}+\operatorname{Err}_{\phi} .
\end{aligned}
$$


Consequently,

$$
\begin{aligned}
\|\underline{\mu}\|_{\mathcal{L}_{(\mathrm{sc})}^{2}(u, \underline{u})} \lesssim \delta^{1 / 2}\left\|\nabla_{3} \underline{\hat{\chi}}\right\|_{\operatorname{Tr}_{(\mathrm{sc})}(\underline{H})}\left\|\nabla^{2}\left({ }^{(3)} \phi+{ }^{(3)} \underline{\phi}+\underline{\widehat{\chi}}\right)\right\|_{\mathcal{L}_{(\mathrm{sc})}^{2}(\underline{H})} \\
+\delta^{1 / 2}\left\|\nabla_{3}\left({ }^{\left({ }^{3}\right)} \phi+{ }^{(3)} \underline{\phi}\right)\right\|_{\operatorname{Tr}_{(\mathrm{sc})}(\underline{H})}\left\|\nabla^{2} \underline{\widehat{\chi}}\right\|_{\mathcal{L}_{(\mathrm{sc})}^{2}(\underline{H})} \\
+\delta^{1 / 2}\left\|\nabla_{3}(\eta+\underline{\eta})\right\|_{\operatorname{Tr}_{(\mathrm{sc})}(\underline{H})}\left\|\nabla^{2} \underline{\eta}\right\|_{\mathcal{L}_{(\mathrm{sc})}^{2}(\underline{H})} \\
+\delta^{1 / 2}\|\nabla \underline{\hat{\chi}}\|_{\operatorname{Tr}_{(\mathrm{sc})}(\underline{H})}\left\|\nabla^{2}(\eta+\underline{\eta})\right\|_{\mathcal{L}_{(\mathrm{sc})}^{2}(\underline{H})}+\operatorname{Err}_{\phi} .
\end{aligned}
$$

We recall from the previous subsection, see Lemma 11.4, that

$$
\left\|\nabla_{3} \underline{\hat{\chi}}\right\|_{\operatorname{Tr}_{(\mathrm{sc})}(\underline{H})} \lesssim C \delta^{-1 / 2}
$$

with a constant $C$ depending only on $\mathcal{O}^{(0)}, \mathcal{R}, \underline{\mathcal{R}}$ as well as $\| \nabla_{3} \underline{\alpha}_{\mathcal{L}_{(\mathrm{sc})}^{2}(\underline{H})}$. Also, from the previous subsection, we have (see Proposition 11.5)

$$
\|\nabla \underline{\hat{\chi}}\|_{\operatorname{Tr}_{(\mathrm{sc})}(\underline{H})} \lesssim C .
$$

Also, in view of (11.38),

$$
\left\|\nabla_{3}\left({ }^{(3)} \phi,{ }^{(3)} \underline{\phi}\right)\right\|_{\operatorname{Tr}_{(\mathrm{sc})}(\underline{H})} \lesssim\left\|\nabla^{2} \underline{\mu}\right\|_{\mathcal{L}_{(\mathrm{sc})}^{2}(\underline{H})}+C .
$$

Also, we can easily show, with the help of the trace estimates of Proposition 11.1 and our Ricci-coefficient estimates, that

$$
\left\|\nabla_{3}(\eta, \underline{\eta})\right\|_{\mathcal{L}_{(\mathrm{sc})}^{2}(\underline{H})} \lesssim C
$$

Consequently,

$$
\|\underline{\mu}\|_{\mathcal{L}_{(\mathrm{sc})}^{2}(u, \underline{u})} \lesssim\|\underline{\mu}\|_{\mathcal{L}_{(\mathrm{sc})}^{2}(0, \underline{u})}+1+C \delta^{1 / 2}\left\|\nabla^{2} \underline{\mu}\right\|_{\mathcal{L}_{(\mathrm{sc})}^{2}(\underline{H})}
$$

On the other hand,

$$
\begin{gathered}
\|\underline{\mu}\|_{\mathcal{L}_{(\mathrm{sc})}^{2}(u, \underline{u})} \lesssim\|\Delta \underline{\mu}\|_{\mathcal{L}_{(\mathrm{sc})}^{2}(u, \underline{u})}+\left\|\nabla^{2} \underline{\omega}\right\|_{\mathcal{L}_{(\mathrm{sc})}^{2}(u, \underline{u})}+C \delta^{1 / 2}\left\|\nabla^{2} \eta\right\|_{\mathcal{L}_{(\mathrm{sc})}^{2}(u, \underline{u})} \\
+C \delta^{1 / 2}\left\|\nabla^{2} \underline{\widehat{\chi}}\right\|_{\mathcal{L}_{(\mathrm{sc})}^{2}(u, \underline{u})} .
\end{gathered}
$$

Hence,

$$
\begin{gathered}
\|\Delta \underline{\mu}\|_{\mathcal{L}_{(\mathrm{sc})}^{2}(u, \underline{u})} \lesssim\|\underline{\mu}\|_{\mathcal{L}_{(\mathrm{sc})}^{2}(0, \underline{u})}+\left\|\nabla^{2} \underline{\omega}\right\|_{\mathcal{L}_{(\mathrm{sc})}^{2}(u, \underline{u})}+C \delta^{1 / 2}\left\|\nabla^{2} \eta\right\|_{\mathcal{L}_{(\mathrm{sc})}^{2}(u, \underline{u})} \\
+\left\|\nabla^{2} \underline{\mu}\right\|_{\mathcal{L}_{(\mathrm{sc})}^{2}(\underline{H})}+C \delta^{1 / 2}\left\|\nabla^{2} \underline{\mu}\right\|_{\mathcal{L}_{(\mathrm{sc})}^{2}(\underline{H})} .
\end{gathered}
$$

We can now proceed precisely as in the last part of the proof of Proposition 11.5 to deduce, after applying elliptic estimates and integrating, that

$$
\left\|\nabla^{2} \underline{\mu}_{\mathcal{L}_{(\mathrm{sc})}^{2}\left(\underline{H}_{\underline{u}}^{(0, u)}\right)} \lesssim \mathcal{O}^{(0)}+\left(1+C \delta^{1 / 2}\right) \int_{0}^{u}\right\| \nabla^{2} \underline{\mu}_{\mathcal{L}_{(\mathrm{sc})}^{2}\left(\underline{H}_{\underline{u}}^{\left(0, u^{\prime}\right)}\right)} d u^{\prime}+C,
$$

from which the desired estimate follows. We have thus proved the second part of the following result. 
Proposition 11.7. The following estimates hold true with a constant $C$ depending on $\mathcal{O}^{(0)}, \mathcal{R}, \underline{\mathcal{R}}$ as well as $\sup _{u}\left\|\nabla_{4} \alpha\right\|_{\mathcal{L}_{(\mathrm{sc})}^{2}\left(H_{u}\right)}$ and $\sup _{\underline{u}}\left\|\nabla_{3} \underline{\alpha}\right\|_{\mathcal{L}_{(\mathrm{sc})}^{2}\left(\underline{H}_{\underline{u}}\right)}$.

(1) We have, along $H=H_{u}$,

$$
\|\nabla(\eta, \underline{\eta})\|_{\operatorname{Tr}_{(\mathrm{sc})}(H)} \lesssim C .
$$

(2) We have, along $\underline{H}=\underline{H}_{\underline{u}}$,

$$
\|\nabla(\eta, \underline{\eta})\|_{\operatorname{Tr}_{(\mathrm{sc})}(\underline{H})} \lesssim C .
$$

(3) Also,

$$
\sup _{S}\|\nabla(\eta, \underline{\eta})\|_{\mathcal{L}_{(\mathrm{sc})}^{4}(S)} \lesssim C
$$

\subsection{Refined estimate for ${ }^{(3)} \phi$}

We end this section by establishing a more refined estimate on ${ }^{(3)} \phi$. This estimate is needed in the argument for the formation of a trapped surface described in our introduction. We examine the equation

$$
\nabla_{3}^{(3)} \phi=\nabla \eta
$$

Commuting with $\nabla$, we obtain

$$
\nabla_{3} \nabla^{(3)} \phi=\left(\operatorname{tr} \underline{\chi}_{0}+\psi\right) \nabla^{(3)} \phi+(\Psi+\psi \psi)^{(3)} \phi+\nabla^{2} \eta \text {. }
$$

Taking into account the triviality of the data for $\nabla^{(3)} \phi$, the non-anomalous estimates for $\Psi$ appearing in this equation and Grönwall's inequality, we obtain

$$
\left\|\nabla^{(3)} \phi\right\|_{\mathcal{L}_{(\mathrm{sc})}^{2}(S)} \lesssim\left\|\nabla^{2} \eta\right\|_{\mathcal{L}_{(\mathrm{sc})}^{2}\left(\underline{H}_{\underline{u}}\right)}+\delta^{1 / 2} C .
$$

Using Proposition 7.3, we get

$$
\left\|\nabla^{(3)} \phi\right\|_{\mathcal{L}_{(\mathrm{sc})}^{2}(S)} \lesssim\|\nabla \varrho\|_{\mathcal{L}_{(\mathrm{sc})}^{2}\left(\underline{H}_{\underline{u}}\right)}+\|\nabla \sigma\|_{\mathcal{L}_{(\mathrm{sc})}^{2}\left(\underline{H}_{\underline{u}}\right)}+\delta^{1 / 4} C .
$$

Combining with the interpolation estimates

$$
\begin{aligned}
\left\|^{(3)} \phi\right\|_{\mathcal{L}_{(\mathrm{sc})}^{\infty}(S)} & \lesssim\left\|^{(3)} \phi\right\|_{\mathcal{L}_{(\mathrm{sc})}^{4}(S)}^{1 / 2}\left\|\nabla^{(3)} \phi\right\|_{\mathcal{L}_{(\mathrm{sc})}^{4}(S)}^{1 / 2}+\delta^{1 / 4}\left\|^{(3)} \phi\right\|_{\mathcal{L}_{(\mathrm{sc})}^{4}(S)}, \\
\left\|\nabla^{(3)} \phi\right\|_{\mathcal{L}_{(\mathrm{sc})}^{4}(S)} & \lesssim\left\|\nabla^{(3)} \phi\right\|_{\mathcal{L}_{(\mathrm{sc})}^{2}(S)}^{1 / 2}\left\|\nabla^{2}\left({ }^{(3)} \phi\right)\right\|_{\mathcal{L}_{(\mathrm{sc})}^{2}(S)}^{1 / 2}+\delta^{1 / 4}\left\|\nabla^{(3)} \phi\right\|_{\mathcal{L}_{(\mathrm{sc})}^{2}(S)}
\end{aligned}
$$

we conclude the following result.

Proposition 11.8. The solution ${ }^{(3)} \phi$ of the problem $\nabla_{3}{ }^{(3)} \phi=\nabla \eta$ with trivial initial data satisfies

$$
\left\|^{(3)} \phi\right\|_{\mathcal{L}_{(\mathrm{sc})}^{\infty}(S)} \lesssim C\left(\|\nabla \varrho\|_{\mathcal{L}_{(\mathrm{sc})}^{2}\left(\underline{H}_{\underline{u}}\right)}+\|\nabla \sigma\|_{\mathcal{L}_{(\mathrm{sc})}^{2}\left(\underline{H}_{\underline{u}}\right)}\right)^{1 / 4}+C \delta^{1 / 16} .
$$




\section{Trace estimates for curvature}

Proposition 12.1. Under the assumptions of finiteness of the norms $\mathcal{R}$ and $\underline{\mathcal{R}}$, which include $\left\|\nabla_{3} \underline{\alpha}\right\|_{\mathcal{L}_{\text {(sc) }}^{2}\left(\underline{H}_{\underline{u}}\right)}$ and the anomalous norm $\left\|\nabla_{4} \alpha\right\|_{\mathcal{L}_{\text {(sc) }}^{2}\left(H_{u}\right)}$, we have

$$
\begin{aligned}
\|\alpha\|_{\operatorname{Tr}_{(\mathrm{sc})}(H)} & \leqslant \delta^{-1 / 4} C, \\
\|(\beta, \varrho, \sigma)\|_{\operatorname{Tr}_{(\mathrm{sc})}(H)} & \leqslant C, \\
\|(\varrho, \sigma, \underline{\beta})\|_{\operatorname{Tr}_{(\mathrm{sc})}(\underline{H})} & \leqslant C, \\
\|\underline{\alpha}\|_{\operatorname{Tr}_{(\mathrm{sc})}(\underline{H})} & \leqslant \delta^{-1 / 4} C .
\end{aligned}
$$

The proof is based on the application of the trace inequalities of Proposition 11.1 and the null structure equations (3.1) and (3.3)-(3.5). According to these, the curvature components $\Psi_{4} \in\{\alpha, \beta, \varrho, \sigma\}$ can be expressed in the form

$$
\Psi_{4}=\nabla_{4} \phi_{4}+\phi \phi
$$

while $\Psi_{3} \in\{\varrho, \sigma, \underline{\alpha}, \underline{\beta}\}$ can be represented as

$$
\Psi_{3}=\nabla_{3} \phi_{3}+\left(\operatorname{tr} \underline{\chi}_{0}\right) \phi+\phi \phi,
$$

$\operatorname{with}\left({ }^{19}\right) \phi_{4} \in\{\hat{\chi}, \eta,\langle\underline{\omega}\rangle\}$ and $\phi_{3} \in\{\langle\omega\rangle, \underline{\hat{\chi}}, \underline{\eta}\}$.

Therefore,

$$
\begin{aligned}
&\left\|\Psi_{4}\right\|_{\operatorname{Tr}_{(\mathrm{sc})}(H)} \lesssim\left\|\nabla_{4} \phi_{4}\right\|_{\operatorname{Tr}_{(\mathrm{sc})}(H)}+\delta^{1 / 2}\|\phi\|_{\mathcal{L}_{\text {(sc) }}^{\infty}}^{2}, \\
&\left\|\Psi_{3}\right\|_{\operatorname{Tr}_{(\mathrm{sc})}(\underline{H})} \lesssim\left\|\nabla_{3} \phi_{3}\right\|_{\operatorname{Tr}_{(\mathrm{sc})}(H)}+\left(1+\delta^{1 / 2}\|\phi\|_{\left.\mathcal{L}_{(\mathrm{sc})}^{\infty}\right)}\right)\|\phi\|_{\mathcal{L}_{(\mathrm{sc})}^{\infty}}^{\infty} .
\end{aligned}
$$

By Proposition 11.1, we have

$$
\begin{aligned}
& \left\|\nabla_{4} \phi_{4}\right\|_{\operatorname{Tr}_{\text {(sc) }}(H)} \lesssim\left(\left\|\nabla_{4}^{2} \phi_{4}\right\|_{\mathcal{L}_{\text {(sc) }}^{2}(H)}+\left\|\phi_{4}\right\|_{\mathcal{L}_{\text {(sc) }}^{2}(H)}+\delta^{1 / 2} C\left(\left\|\phi_{4}\right\|_{\mathcal{L}_{\text {(sc) }}^{\infty}}+\left\|\nabla_{4} \phi_{4}\right\|_{\mathcal{L}_{\text {(sc) }}^{4}(S)}\right)\right)^{1 / 2} \\
& \times\left(\left\|\nabla^{2} \phi_{4}\right\|_{\mathcal{L}_{(\mathrm{sc})}^{2}(H)}+\delta^{1 / 2} C\left(\left\|\phi_{4}\right\|_{\mathcal{L}_{\text {(sc) }}^{\infty}}+\left\|\nabla \phi_{4}\right\|_{\mathcal{L}_{(\mathrm{sc})}^{4}(S)}\right)\right)^{1 / 2} \\
& +\left\|\nabla_{4} \nabla \phi_{4}\right\|_{\mathcal{L}_{\text {(sc) }}^{2}(H)}+\left\|\phi_{4}\right\|_{\mathcal{L}_{\text {(sc) }}^{\infty}}+\left\|\nabla \phi_{4}\right\|_{\mathcal{L}_{\text {(sc) }}^{2}(H)}, \\
& \left\|\nabla_{3} \phi_{3}\right\|_{\operatorname{Tr}_{(\mathrm{sc})}(\underline{H})} \lesssim\left(\left\|\nabla_{3}^{2} \phi_{3}\right\|_{\mathcal{L}_{\text {(sc) }}^{2}(\underline{H})}+\left\|\phi_{3}\right\|_{\mathcal{L}_{\text {(sc) }}^{2}(\underline{H})}+\delta^{1 / 2} C\left(\left\|\phi_{3}\right\|_{\mathcal{L}_{\text {(sc) }}^{\infty}}+\left\|\nabla_{3} \phi_{3}\right\|_{\mathcal{L}_{(\text {sc })}^{4}(S)}\right)\right)^{1 / 2} \\
& \times\left(\left\|\nabla^{2} \phi_{3}\right\|_{\mathcal{L}_{(\mathrm{sc})}^{2}(\underline{H})}+\delta^{1 / 2} C\left(\left\|\phi_{3}\right\|_{\mathcal{L}_{(\mathrm{sc})}^{\infty}}+\left\|\nabla \phi_{3}\right\|_{\mathcal{L}_{(\mathrm{sc})}^{4}(S)}\right)\right)^{1 / 2} \\
& +\left\|\nabla_{3} \nabla \phi_{3}\right\|_{\mathcal{L}_{\text {(sc) }}^{2}(\underline{H})}+\left\|\phi_{3}\right\|_{\mathcal{L}_{\text {(sc) }}^{\infty}}+\left\|\nabla \phi_{3}\right\|_{\mathcal{L}_{\text {(sc) }}^{2}(\underline{H})} .
\end{aligned}
$$

We observe that all the involved norms, with the exception of

$$
\left\|\nabla_{4}^{2} \phi_{4}\right\|_{\mathcal{L}_{(\mathrm{sc})}^{2}(H)} \quad \text { and } \quad\left\|\nabla_{3}^{2} \phi_{3}\right\|_{\mathcal{L}_{(\mathrm{sc})}^{2}(\underline{H})},
$$

have already been estimated.

$\left({ }^{19}\right)$ Recall that $\langle\underline{\omega}\rangle=\left(\underline{\omega}, \underline{\omega}^{\dagger}\right)$ and $\langle\omega\rangle=\left(-\omega, \omega^{\dagger}\right)$, see (6.15) and (6.16). 
Recall that the derivatives with no estimates are the $\mathcal{L}_{(\mathrm{sc})}^{4}(S)$ norms of $\nabla_{4} \omega$ and $\nabla_{3} \underline{\omega}$, and either the $\mathcal{L}_{\text {(sc) }}^{2}(H)$ or the $\mathcal{L}_{\text {(sc) }}^{2}(\underline{H})$ norms of $\nabla \nabla_{4} \omega$ and $\nabla \nabla_{3} \underline{\underline{\omega}}$, while $\nabla \nabla_{4} \hat{\chi}$ and $\nabla \nabla_{3} \underline{\hat{\chi}}$ are controlled only along $H$ and $\underline{H}$, respectively. Finally, the $\mathcal{L}_{(\mathrm{sc})}^{2}(S)$ and $\mathcal{L}_{\text {(sc) }}^{4}(S)$ estimates for $\hat{\chi}, \underline{\hat{\chi}}, \nabla_{3,4} \widehat{\chi}$ and $\nabla_{3,4} \underline{\widehat{\chi}}$ are $\delta^{-1 / 2}$ and $\delta^{-1 / 4}$ anomalous. Therefore, for $\phi_{4}=\hat{\chi}$, i.e. $\Psi_{4}=\alpha$,

$$
\left\|\nabla_{4} \widehat{\chi}\right\|_{\operatorname{Tr}_{(\mathrm{sc})}(H)} \lesssim C\left(\left\|\nabla_{4}^{2} \widehat{\chi}\right\|_{\mathcal{L}_{(\mathrm{sc})}^{2}(H)}+C \delta^{-1 / 2}\right)^{1 / 2}+C,
$$

and for $\phi_{3}=\underline{\hat{\chi}}$, i.e. $\Psi_{3}=\underline{\alpha}$,

$$
\left\|\nabla_{3} \underline{\hat{\chi}}\right\|_{\operatorname{Tr}_{(\mathrm{sc})}(\underline{H})} \lesssim C\left(\left\|\nabla_{3}^{2} \underline{\hat{\chi}}\right\|_{\mathcal{L}_{(\mathrm{sc})}^{2}(\underline{H})}+C \delta^{-1 / 2}\right)^{1 / 2}+C .
$$

The remaining $\phi_{4}$ and $\phi_{3}$ satisfy

$$
\begin{aligned}
&\left\|\nabla_{4} \phi_{4}\right\|_{\operatorname{Tr}_{(\mathrm{sc})}(H)} \lesssim C\left(\left\|\nabla_{4}^{2} \phi_{4}\right\|_{\mathcal{L}_{(\mathrm{sc})}^{2}(H)}+C\right)^{1 / 2}+C, \\
&\left\|\nabla_{3} \phi_{3}\right\|_{\operatorname{Tr}_{(\mathrm{sc})}(\underline{H})} \lesssim C\left(\left\|\nabla_{4}^{2} \phi_{3}\right\|_{\mathcal{L}_{(\mathrm{sc})}^{2}(\underline{H})}+C\right)^{1 / 2}+C .
\end{aligned}
$$

We now express

$$
\nabla_{4}^{2} \phi_{4}=\nabla_{4} \Psi_{4}+\nabla_{4} \phi \phi \quad \text { and } \quad \nabla_{3}^{2} \phi_{3}=\nabla_{3} \Psi_{3}+\nabla_{3} \phi \psi
$$

Therefore,

$$
\begin{aligned}
\left\|\nabla_{4}^{2} \phi_{4}\right\|_{\mathcal{L}_{\text {(sc) }}^{2}(H)} & \lesssim\left\|\nabla_{4} \Psi_{4}\right\|_{\mathcal{L}_{\text {(sc) }}^{2}(H)}+\delta^{1 / 2}\left\|\nabla_{4} \phi\right\|_{\mathcal{L}_{\text {(sc) }}^{2}(H)}\|\phi\|_{\mathcal{L}_{\text {(sc) }}^{\infty}} \lesssim\left\|\nabla_{4} \Psi_{4}\right\|_{\mathcal{L}_{\text {(sc) }}^{2}(H)}+C, \\
\left\|\nabla_{3}^{2} \phi_{3}\right\|_{\mathcal{L}_{\text {(sc) }}^{2}(\underline{H})} & \lesssim\left\|\nabla_{3} \Psi_{3}\right\|_{\mathcal{L}_{\text {(sc) }}^{2}(\underline{H})}+\delta^{1 / 2}\left\|\nabla_{3} \phi\right\|_{\mathcal{L}_{\text {(sc) }}^{2}(H)}\|\phi\|_{\mathcal{L}_{\text {(sc) }}^{\infty}} \lesssim\left\|\nabla_{4} \Psi_{4}\right\|_{\mathcal{L}_{\text {(sc) }}^{2}(\underline{H})}+C,
\end{aligned}
$$

where we took into account possible $\delta^{-1 / 2}$ anomalies of $\left\|\nabla_{4} \phi\right\|_{\mathcal{L}_{(\mathrm{sc})}^{2}(H)}$ and $\left\|\nabla_{3} \phi\right\|_{\mathcal{L}_{(\mathrm{sc})}^{2}(\underline{H})}$. These immediately yield the desired trace estimates for $\alpha$ and $\underline{\alpha}$. For the remaining components $\Psi_{4}$ and $\Psi_{3}$ we may express from the Bianchi identities

$$
\nabla_{4} \Psi_{4}=\nabla \Psi^{4}+\phi \Psi \quad \text { and } \quad \nabla_{3} \Psi_{3}=\nabla \Psi^{3}+\left(\operatorname{tr} \underline{\chi}_{0}\right) \Psi+\phi \Psi,
$$

where $\Psi^{4} \in\{\alpha, \beta\}$ and $\Psi^{3} \in\{\underline{\alpha}, \underline{\beta}\}$. Therefore,

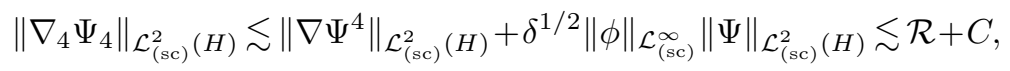

$$
\begin{aligned}
& \left\|\nabla_{3} \Psi_{3}\right\|_{\mathcal{L}_{\text {(sc) }}^{2}(\underline{H})} \lesssim\left\|\nabla \Psi^{3}\right\|_{\mathcal{L}_{\text {(sc) }}^{2}(\underline{H})}+\left(1+\delta^{1 / 2}\|\phi\|_{\left.\mathcal{L}_{\text {(sc) }}^{\infty}\right)}\right)\|\Psi\|_{\mathcal{L}_{\text {(sc) }}^{2}(\underline{H})} \lesssim \mathcal{R}+C .
\end{aligned}
$$

In the last step we have to be careful to avoid the double anomalous term $\left(\operatorname{tr} \underline{\chi}_{0}\right) \alpha$. Its appearance is prohibited by the signature considerations, according to which

$$
1 \geqslant \operatorname{sgn}\left(\nabla_{3} \Psi_{3}\right)=\operatorname{sgn}\left(\left(\operatorname{tr} \underline{\chi}_{0}\right) \alpha\right)=2 .
$$




\section{Estimates for the rotation vector fields}

We define the algebra of rotation vector fields ${ }^{(i)} O$ obeying the commutation relations

$$
\left[{ }^{(i)} O,{ }^{(j)} O\right]=\epsilon_{i j k}{ }^{(k)} O
$$

obtained by parallel transport of the standard rotation vector fields on $\mathbb{S}^{2}=S_{u, 0} \subset H_{u, 0}$ along the integral curves of $e_{4}$. Suppressing the index ${ }^{(i)}$ we obtain

$$
\nabla_{4} O_{b}=\chi_{b c} O_{c}
$$

Commuting with $\nabla$ and $\nabla_{3}$, we obtain

$$
\begin{aligned}
\nabla_{4} \nabla O & =\chi \nabla O+\beta O+(\nabla \chi) O+\chi \underline{\eta} O, \\
\nabla_{4} \nabla_{3} O & =(\underline{\eta}-\eta) \nabla O+(\chi+\omega) \nabla_{3} O+\sigma O+(\underline{\omega} \chi+\eta \underline{\eta}) O+\left(\nabla_{3} \chi\right) O .
\end{aligned}
$$

The only non-trivial components of the deformation tensor $\pi_{\alpha \beta}=\frac{1}{2}\left(\nabla_{\alpha} O_{\beta}+\nabla_{\beta} O_{\alpha}\right)$ are

$$
\pi_{34}=-2(\eta+\underline{\eta})_{a} O_{a}, \quad \pi_{a b}=\frac{1}{2}\left(\nabla_{a} O_{b}+\nabla_{b} O_{a}\right) \quad \text { and } \quad \pi_{3 a}=\frac{1}{2}\left(\nabla_{3} O_{a}-\underline{\chi}_{a b} O_{b}\right):=\frac{1}{2} Z_{a} .
$$

\subsection{Estimates for $H$ and $Z$}

The quantity $Z$ satisfies the following transport equation, $\left({ }^{20}\right)$ written schematically

$$
\nabla_{4} Z=(\nabla(\eta+\underline{\eta})) O+(\underline{\eta}-\eta) \nabla O+\omega Z+(\sigma+\varrho) O+(\eta-\underline{\eta})(\eta+\underline{\eta}) O .
$$

Let $H_{a b}=\nabla_{a} O_{b}$ denote the non-symmetrized derivative of $O$. Then,

$$
\nabla_{4} H=\chi H+\beta O+(\nabla \chi) O+\chi \underline{\eta} O
$$

We now rewrite these equations schematically in the form

$$
\begin{aligned}
\nabla_{4} Z & =\left(\nabla \psi_{34}\right) O+\psi_{34} H+(\chi+\omega) Z+\Psi_{g} O+\psi_{34} \psi_{34} O \\
\nabla_{4} H & =\psi H+\left(\Theta_{4}+\nabla \psi_{4}\right) O+\psi \psi_{34} O
\end{aligned}
$$

Here $\psi_{34} \in\{\eta, \underline{\eta}\}$ and $\Psi_{g} \in\{\varrho, \sigma\}$. In what follows $\psi_{34}$ will be treated either as a $\psi_{3}$ or a $\psi_{4}$ quantity, depending on the situation. The quantities $H$ and $Z$ can be assigned signature

$\left({ }^{20}\right)$ Note the absence of $\underline{\chi}$ and $\underline{\omega}$. 
and scaling (consistent with those for the Ricci-coefficient and curvature components) according to

$$
\operatorname{sgn}(H)-\frac{1}{2}=\operatorname{sc}(H)=0 \quad \text { and } \quad \operatorname{sgn}(Z)-\frac{1}{2}=\operatorname{sc}(Z)=-\frac{1}{2} .
$$

In view of equations (13.1) we derive, by integration,

$$
\|Z\|_{\mathcal{L}_{(\mathrm{sc})}^{\infty}} \lesssim\left\|\nabla \psi_{4}\right\|_{\operatorname{Tr}_{(\mathrm{sc})}}+\left\|\Psi_{g}\right\|_{\operatorname{Tr}_{(\mathrm{sc})}}+\delta^{1 / 2}\|\psi\|_{\mathcal{L}_{(\mathrm{sc})}^{\infty}}\left(\|\psi\|_{\mathcal{L}_{(\mathrm{sc})}^{\infty}}+\|H\|_{\mathcal{L}_{(\mathrm{sc})}^{\infty}}+\|Z\|_{\mathcal{L}_{(\mathrm{sc})}^{\infty}}\right)
$$

Thus, according to the trace estimates of Proposition 11.7 for $\psi_{4} \in\{\eta, \underline{\eta}\}$ and Proposition 12.1 for $\Psi_{g}$, we derive

$$
\|Z\|_{\mathcal{L}_{(\mathrm{sc})}^{\infty}} \lesssim C+\delta^{1 / 2} C\left(\|H\|_{\mathcal{L}_{(\mathrm{sc})}^{\infty}}+\|Z\|_{\mathcal{L}_{(\mathrm{sc})}^{\infty}}\right)
$$

Similarly,

$$
\begin{aligned}
\|H\|_{\mathcal{L}_{(\mathrm{sc})}^{\infty}} & \lesssim\left\|\nabla \psi_{4}\right\|_{\operatorname{Tr}_{(\mathrm{sc})}}+\left\|\Theta_{4}\right\|_{\mathcal{L}_{(\mathrm{sc})}^{\infty}}+\delta^{1 / 2}\|\psi\|_{\mathcal{L}_{(\mathrm{sc})}^{\infty}}^{2}\left(\|\psi\|_{\mathcal{L}_{(\mathrm{sc})}^{\infty}}+\|H\|_{\mathcal{L}_{(\mathrm{sc})}^{\infty}}\right) \\
& \lesssim C+\delta^{1 / 2} C\left(C+\|H\|_{\left.\mathcal{L}_{(\mathrm{sc})}^{\infty}\right) .}\right.
\end{aligned}
$$

Therefore we have proved $\left({ }^{21}\right)$ the following result.

Proposition 13.1. The quantities $Z$ and $H$ satisfy the estimates

$$
\|H\|_{\mathcal{L}_{(\mathrm{sc})}^{\infty}}+\|Z\|_{\mathcal{L}_{(\mathrm{sc})}^{\infty}} \lesssim C
$$

with a constant $C=C\left(\mathcal{I}^{(0)}, \mathcal{R}_{[1]}, \underline{\mathcal{R}}_{[1]}\right)$.

We add a small remark concerning the symmetrized $\nabla$ derivatives of $O$.

Proposition 13.2. Let

$$
H_{a b}^{(s)}:=\nabla_{a} O_{b}+\nabla_{a} O_{b}=H_{a b}+H_{b a} .
$$

Then, in addition to all the estimates for $H, H^{(s)}$ also enjoys the non-anomalous $\mathcal{L}_{(\mathrm{sc})}^{2}(S)$ estimate

$$
\left\|H^{(s)}\right\|_{\mathcal{L}_{(\mathrm{sc})}^{2}(S)} \lesssim C
$$

Similarly,

$$
\|Z\|_{\mathcal{L}_{(\mathrm{sc})}^{2}(S)} \lesssim C .
$$

The result easily follows from the transport equation for $H^{\prime}$, which is virtually the same as for $H$, and, crucially, from the triviality of the initial data for $H^{s}$. The claim for $Z$ follows from the same considerations.

$\left({ }^{21}\right)$ Note the triviality of the data for $Z$ on $\underline{H}_{0}$. Otherwise the term $\chi O$ in the definition of $Z$ might have caused an $\mathcal{L}_{(\mathrm{sc})}^{\infty}$ anomaly. The data for $H$ however is not trivial. Initially $\|H\|_{L^{\infty} \sim 1 \text {, which }}$ means that while it is anomalous in $\mathcal{L}_{(\mathrm{sc})}^{2}(S)$ it is not in $\mathcal{L}_{(\mathrm{sc})}^{\infty}$. 


\section{2. $\mathcal{L}_{(\mathrm{sc})}^{2}(S)$ estimates for $\nabla H$ and $\nabla Z$}

We prove below the following result.

Proposition 13.3. The following estimates hold true with $C=C\left(\mathcal{I}^{(0)}, \mathcal{R}, \underline{\mathcal{R}}\right)$,

$$
\begin{array}{r}
\|\nabla H\|_{\mathcal{L}_{(\mathrm{sc})}^{2}(S)}+\|\nabla Z\|_{\mathcal{L}_{(\mathrm{sc})}^{2}(S)} \lesssim C, \\
\left\|\nabla_{4} \nabla H\right\|_{\mathcal{L}_{(\mathrm{sc})}^{2}(H)}+\left\|\nabla_{4} \nabla Z\right\|_{\mathcal{L}_{(\mathrm{sc})}^{2}(H)} \lesssim C .
\end{array}
$$

Proof. We first commute the transport equations for $H$ and $Z$ with $\nabla$ :

$$
\begin{gathered}
\nabla_{4} \nabla H=\psi \nabla H+(\nabla \psi) H+\left(\nabla \Theta_{4}+\nabla^{2} \psi_{4}\right) O+\left(\Theta_{4}+\Psi_{g}\right) H \\
+\psi(\nabla \psi) O+\psi_{34} \nabla_{4} H+\psi \psi_{g} H \\
\nabla_{4} \nabla Z=\left(\nabla^{2} \psi_{34}\right) O+\left(\nabla \psi+\Psi_{g}\right)(H+Z)+\psi(\nabla H+\nabla Z)+\left(\nabla \Psi_{g}\right) O \\
+\psi(\nabla \psi) O+\psi \psi(H+Z)+\psi_{34} \nabla_{4} Z .
\end{gathered}
$$

The term $\nabla \Psi_{g}$ is in fact $\nabla(\sigma+\varrho)$. The estimate for $\nabla H$ follows immediately from the following estimates:

$$
\begin{aligned}
& \|\psi \nabla H\|_{\mathcal{L}_{(\mathrm{sc})}^{2}(H)} \lesssim \delta^{1 / 2}\|\psi\|_{\mathcal{L}_{(\mathrm{sc})}^{\infty}}\|\nabla H\|_{\mathcal{L}_{(\mathrm{sc})}^{2}(H)} \lesssim \delta^{1 / 2} C\|\nabla H\|_{\mathcal{L}_{(\mathrm{sc})}^{2}(H)} \\
& \|(\nabla \psi) H\|_{\mathcal{L}_{(\mathrm{sc})}^{2}(H)} \lesssim \delta^{1 / 2}\|H\|_{\mathcal{L}_{(\mathrm{sc})}^{\infty}}\|\nabla \psi\|_{\mathcal{L}_{(\mathrm{sc})}^{2}(H)} \lesssim \delta^{1 / 2} C \\
& \|(\nabla \Theta) O\|_{\mathcal{L}_{(\mathrm{sc})}^{2}(H)} \lesssim\|\nabla \Theta\|_{\mathcal{L}_{(\mathrm{sc})}^{2}(H)} \lesssim C, \\
& \left\|\left(\nabla^{2} \psi\right) O\right\|_{\mathcal{L}_{(\mathrm{sc})}^{2}(H)} \lesssim\left\|\nabla^{2} \psi\right\|_{\mathcal{L}_{(\mathrm{sc})}^{2}(H)} \lesssim C, \\
& \left\|\left(\Theta+\Psi_{g}\right) H\right\|_{\mathcal{L}_{(\mathrm{sc})}^{2}(H)} \lesssim \delta^{1 / 2}\|H\|_{\mathcal{L}_{(\mathrm{sc})}^{\infty}}\left(\|\Theta\|_{\mathcal{L}_{(\mathrm{sc})}^{2}(H)}+\left\|\Psi_{g}\right\|_{\mathcal{L}_{(\mathrm{sc})}^{2}(H)}\right) \lesssim \delta^{1 / 2} C, \\
& \|\psi(\nabla \psi) O\|_{\mathcal{L}_{(\mathrm{sc})}^{2}(H)} \lesssim \delta^{1 / 2}\|\psi\|_{\mathcal{L}_{(\mathrm{sc})}^{\infty}}\|\nabla \psi\|_{\mathcal{L}_{(\mathrm{sc})}^{2}(H)} \lesssim \delta^{1 / 2} C, \\
& \left\|\psi \psi_{g} H\right\|_{\mathcal{L}_{(\mathrm{sc})}^{2}(H)} \lesssim \delta\|\psi\|_{\mathcal{L}_{(\mathrm{sc})}^{\infty}}\|H\|_{\mathcal{L}_{(\mathrm{sc})}^{\infty}}\left\|\psi_{g}\right\|_{\mathcal{L}_{(\mathrm{sc})}^{2}(H)} \lesssim \delta C, \\
& \left\|\psi \nabla_{4} H\right\|_{\mathcal{L}_{(\mathrm{sc})}^{2}(H)} \lesssim \delta^{1 / 2}\|\psi\|_{\mathcal{L}_{(\mathrm{sc})}^{\infty}}\left\|\nabla_{4} H\right\|_{\mathcal{L}_{(\mathrm{sc})}^{2}(H)} \lesssim \delta^{1 / 2} C .
\end{aligned}
$$

The estimates for $\nabla Z$ are proved in exactly the same manner.

\section{3. $\mathcal{L}_{(\mathrm{sc})}^{4}(S)$ estimates for $\nabla H$ and $\nabla Z$}

The results of the previous proposition can be strengthened to give the following result.

Proposition 13.4. We have

$$
\|\nabla H\|_{\mathcal{L}_{(\mathrm{sc})}^{4}(S)}+\|\nabla Z\|_{\mathcal{L}_{(\mathrm{sc})}^{4}(S)} \lesssim C
$$


Proof. The arguments can be followed almost verbatim, as in the last proposition, with the exception of the analysis of the two terms

$$
\left(\nabla^{2} \psi_{34}\right) O \quad \text { and } \quad\left(\nabla \Psi_{g}\right) O=(\nabla(\sigma+\varrho)) O
$$

We recall that $\psi_{34} \in\{\eta, \underline{\eta}\}$ and, according to Proposition 11.6, we can write

$$
\nabla \psi_{43}=\nabla_{4} \phi
$$

with $\phi$ satisfying the estimates

$$
\begin{gathered}
\left\|\nabla^{2} \phi\right\|_{\mathcal{L}_{(\mathrm{sc})}^{2}(H)}+\left\|\nabla^{2} \phi\right\|_{\mathcal{L}_{(\mathrm{sc})}^{2}(\underline{H})}+\|\nabla \phi\|_{\mathcal{L}_{(\mathrm{sc})}^{2}(S)}+\|\phi\|_{\mathcal{L}_{(\mathrm{sc})}^{2}(S)} \leqslant C, \\
\left\|\nabla_{4} \phi\right\|_{\mathcal{L}_{(\mathrm{sc})}^{2}(S)}+\left\|\nabla_{4} \nabla \phi_{4}\right\|_{\mathcal{L}_{(\mathrm{sc})}^{2}(S)}+\|\phi\|_{\mathcal{L}_{(\mathrm{sc})}^{\infty}}+\|\nabla \phi\|_{\mathcal{L}_{(\mathrm{sc})}^{4}(S)} \lesssim C .
\end{gathered}
$$

We now write

$$
\begin{aligned}
\nabla^{2} \psi_{43} O & =\nabla_{4}((\nabla \phi) O)-(\nabla \phi) \chi O-\left[\nabla_{4}, \nabla\right] \phi O \\
& =\nabla_{4}((\nabla \phi) O)+\chi(\nabla \phi) O+\Psi_{g} \phi O+\psi(\nabla \psi) O+\psi \psi \phi O
\end{aligned}
$$

We estimate

$$
\begin{gathered}
\delta^{-1} \int_{0}^{\underline{u}}\|(\nabla \phi) \chi O\|_{\mathcal{L}_{(\mathrm{sc})}^{4}\left(S_{u, \underline{u}}\right)} d \underline{u}^{\prime} \lesssim \delta^{1 / 2} \sup _{\underline{u}}\|\nabla \phi\|_{\mathcal{L}_{(\mathrm{sc})}^{4}\left(S_{u, \underline{u}}\right)}\|\chi\|_{\mathcal{L}_{(\mathrm{sc})}^{\infty}} \lesssim \delta^{1 / 2} C, \\
\delta^{-1} \int_{0}^{\underline{u}}\left\|\Psi_{g} \phi O\right\|_{\mathcal{L}_{(\mathrm{sc})}^{4}\left(S_{u, \underline{u}}\right)} d \underline{u}^{\prime} \\
\quad \lesssim \delta^{1 / 2}\left(\left\|\nabla \Psi_{g}\right\|_{\mathcal{L}_{(\mathrm{sc})}^{2}(H)}^{1 / 2}\left\|\Psi_{g}\right\|_{\mathcal{L}_{(\mathrm{sc})}^{2}(H)}^{1 / 2}+\delta^{1 / 4}\left\|\Psi_{g}\right\|_{\mathcal{L}_{(\mathrm{sc})}^{2}(H)}\right)\|\phi\|_{\mathcal{L}_{(\mathrm{sc})}^{\infty}} \lesssim \delta^{1 / 2} C, \\
\delta^{-1} \int_{0}^{\underline{u}}\|(\nabla \psi) \psi O\|_{\mathcal{L}_{(\mathrm{sc})}^{4}\left(S_{u, \underline{u}}\right)} d \underline{u}^{\prime} \lesssim \delta^{1 / 2} \sup _{\underline{u}}\|\nabla \psi\|_{\mathcal{L}_{(\mathrm{sc})}^{4}\left(S_{u, \underline{u}}\right)}\|\psi\|_{\mathcal{L}_{(\mathrm{sc})}^{\infty}} \lesssim \delta^{1 / 2} C, \\
\delta^{-1} \int_{0}^{\underline{u}}\|\psi \psi \phi O\|_{\mathcal{L}_{(\mathrm{sc})}^{4}\left(S_{u, \underline{u}}\right)} d \underline{u}^{\prime} \lesssim \delta \sup _{\underline{u}}\|\phi\|_{\mathcal{L}_{(\mathrm{sc})}^{4}\left(S_{u, \underline{u}}\right)}\|\psi\|_{\mathcal{L}_{(\mathrm{sc})}^{\infty}}^{2} \lesssim \delta C .
\end{gathered}
$$

On the other hand, the null structure equations give, for $\langle\underline{\omega}\rangle=\left(\underline{\omega}, \underline{\omega}^{\dagger}\right)$,

$$
\nabla_{4}\langle\underline{\omega}\rangle=(\varrho, \sigma)+\psi_{g} \psi_{g}
$$

As a result,

$$
\nabla(\varrho, \sigma) O=\nabla_{4}((\nabla\langle\underline{\omega}\rangle) O)+\left(\psi \nabla \psi+\chi \nabla\langle\underline{\omega}\rangle+\Psi_{g}\langle\underline{\omega}\rangle+\psi_{g} \Psi_{g}+\psi \psi_{g}\left(\langle\underline{\omega}\rangle+\psi_{g}\right)\right) O .
$$


We can estimate

$$
\begin{aligned}
& \delta^{-1} \int_{0}^{\underline{u}}\|(\nabla\langle\underline{\omega}\rangle) \chi O\|_{\mathcal{L}_{(\mathrm{sc})}^{4}\left(S_{u, \underline{u}}\right)} d \underline{u}^{\prime} \lesssim \delta^{1 / 2} \sup _{\underline{u}}\|\nabla\langle\underline{\omega}\rangle\|_{\mathcal{L}_{(\mathrm{sc})}^{4}\left(S_{u, \underline{u}}\right)}\|\chi\|_{\mathcal{L}_{(\mathrm{sc})}^{\infty}} \lesssim \delta^{1 / 2} C \\
& \delta^{-1} \int_{0}^{\underline{u}}\left\|\Psi_{g} \psi O\right\|_{\mathcal{L}_{(\mathrm{sc})}^{4}\left(S_{u, \underline{u}}\right)} d \underline{u}^{\prime} \\
& \lesssim \delta^{1 / 2}\left(\left\|\nabla \Psi_{g}\right\|_{\mathcal{L}_{(\mathrm{sc})}^{2}(H)}^{1 / 2}\left\|\Psi_{g}\right\|_{\mathcal{L}_{(\mathrm{sc})}^{2}(H)}^{1 / 2}+\delta^{1 / 4}\left\|\Psi_{g}\right\|_{\mathcal{L}_{(\mathrm{sc})}^{2}(H)}\right)\|\psi\|_{\mathcal{L}_{(\mathrm{sc})}^{\infty}} \lesssim \delta^{1 / 2} C, \\
& \delta^{-1} \int_{0}^{\underline{u}}\|(\nabla \psi) \psi O\|_{\mathcal{L}_{(\mathrm{sc})}^{4}\left(S_{u, \underline{u}}\right)} d \underline{u}^{\prime} \lesssim \delta^{1 / 2} \sup _{\underline{u}}\|\nabla \psi\|_{\mathcal{L}_{(\mathrm{sc})}^{4}\left(S_{u, \underline{u}}\right)}\|\psi\|_{\mathcal{L}_{(\mathrm{sc})}^{\infty} \lesssim \delta^{1 / 2} C,} \\
& \delta^{-1} \int_{0}^{\underline{u}}\left\|\psi \psi\left(\langle\underline{\omega}\rangle+\psi_{g}\right) O\right\|_{\mathcal{L}_{(\mathrm{sc})}^{4}\left(S_{u, \underline{u}}\right)} d \underline{u}^{\prime} \lesssim \delta \sup _{\underline{u}^{\prime} \leqslant \underline{u}}\left\|\langle\underline{\omega}\rangle+\psi_{g}\right\|_{\mathcal{L}_{(\mathrm{sc})}^{4}\left(S_{u, \underline{u}^{\prime}}\right)}\|\psi\|_{\mathcal{L}_{(\mathrm{sc})}^{\infty}}^{2} \lesssim \delta C .
\end{aligned}
$$

These allow us to conclude that

$$
\begin{aligned}
& \delta^{-1} \int_{0}^{\underline{u}}\left\|\nabla_{4}[(\nabla H, \nabla Z)-(\nabla \phi) O-(\nabla\langle\underline{\omega}\rangle) O]\right\|_{\mathcal{L}_{(\mathrm{sc})}^{4}\left(S_{u, \underline{u}^{\prime}}\right)} d \underline{u}^{\prime \prime} \\
& \lesssim \delta^{1 / 2} \sup _{\underline{u}^{\prime} \leqslant \underline{u}}\|(\nabla H, \nabla Z)\|_{\mathcal{L}_{(\mathrm{sc})}^{4}\left(S_{u, \underline{u}^{\prime}}\right)}+\delta^{1 / 2} C .
\end{aligned}
$$

Making use of the $\mathcal{L}_{(\mathrm{sc})}^{4}(S)$ bounds on both $\nabla \phi$ and $\nabla\langle\underline{\omega}\rangle$, we finally obtain the estimate

$$
\delta^{-1} \int_{0}^{u}\left\|\nabla_{4}(\nabla H, \nabla Z)\right\|_{\mathcal{L}_{(\mathrm{sc})}^{4}\left(S_{u, \underline{u}^{\prime}}\right)} d u^{\prime} \lesssim \delta^{1 / 2} \sup _{\underline{u}^{\prime} \leqslant \underline{u}}\|(\nabla H, \nabla Z)\|_{\mathcal{L}_{(\mathrm{sc})}^{4}\left(S_{u, \underline{u}^{\prime}}\right)}+C \delta^{1 / 2}
$$

from which the conclusion of the proposition easily follows.

\subsection{Estimates for $\nabla_{3} Z$}

We now examine the equation for $\nabla_{3} Z$ :

$$
\begin{aligned}
\nabla_{4} \nabla_{3} Z=\nabla_{3} \nabla \psi_{34}+\left(\nabla \psi_{34}\right) Z+\left(\nabla \psi_{34}\right) \underline{\chi}+\left(\nabla_{3} \psi_{34}\right) H+\psi_{34} \nabla_{3} H \\
+\left(\nabla_{3} \chi+\nabla_{3} \omega\right) Z+\omega \nabla_{3} Z+\left(\nabla_{3} \Psi_{g}\right) O+(\varrho+\sigma) Z \\
+\Psi_{g} \underline{\chi}+\left(\nabla_{3} \psi_{34}\right) \psi_{34}+\psi_{34} \psi_{34} Z+\psi_{34} \psi_{34} \underline{\chi} .
\end{aligned}
$$

To estimate the right-hand side of this equation we will need to use the first and second derivative estimates for $\psi$ of Propositions 8.1, 8.2, 8.4 and 8.8, keeping in mind possible anomalies of $\underline{\chi}, \nabla_{4} \hat{\chi}, \nabla_{3} \widehat{\chi}$ and $\nabla_{3} \underline{\hat{\chi}}$, the relationship

$$
\nabla_{3}(\varrho+\sigma)=\nabla \underline{\beta}+\left(\operatorname{tr} \underline{\chi}_{0}+\psi\right) \Psi
$$


given by the null Bianchi identities, and the $\mathcal{L}_{(\mathrm{sc})}^{2}(S)$ curvature estimate $\left({ }^{22}\right)$

$$
\|\Psi\|_{\mathcal{L}_{(\mathrm{sc})}^{2}(S)} \leqslant C
$$

of Propositions 6.9 and 6.11. Thus,

$$
\begin{aligned}
& \left\|\nabla_{3} \nabla \psi_{34}\right\|_{\mathcal{L}_{(\mathrm{sc})}^{2}(H)} \lesssim C, \\
& \|(\nabla \psi) Z\|_{\mathcal{L}_{(\mathrm{sc})}^{2}(H)} \lesssim \delta^{1 / 2}\|Z\|_{\mathcal{L}_{\text {(sc) }}^{\infty}}\|\nabla \psi\|_{\mathcal{L}_{\text {(sc) }}^{2}(H)} \lesssim \delta^{1 / 2} C,
\end{aligned}
$$

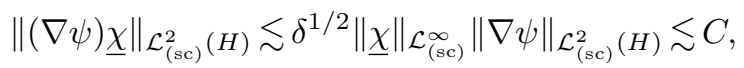

$$
\begin{aligned}
& \left\|\left(\nabla_{3} \psi_{34}\right) H\right\|_{\mathcal{L}_{(\mathrm{sc})}^{2}(H)} \lesssim \delta^{1 / 2}\|H\|_{\mathcal{L}_{\text {(sc) }}^{\infty}}\left\|\nabla_{3} \psi_{34}\right\|_{\mathcal{L}_{(\mathrm{sc})}^{2}(H)} \lesssim \delta^{1 / 2} C, \\
& \left\|\psi \nabla_{3} H\right\|_{\mathcal{L}_{(\mathrm{sc})}^{2}(H)} \lesssim \delta^{1 / 2}\|\psi\|_{\mathcal{L}_{\text {(sc) }}^{\infty}}\left\|\nabla_{3} H\right\|_{\mathcal{L}_{(\mathrm{sc})}^{2}(H)} \lesssim \delta^{1 / 2} C\left\|\nabla_{3} H\right\|_{\mathcal{L}_{(\mathrm{sc})}^{2}(H)}, \\
& \left\|\left(\nabla_{3} \omega\right) Z\right\|_{\mathcal{L}_{\text {(sc) }}^{2}(H)} \lesssim \delta^{1 / 2}\|Z\|_{\mathcal{L}_{\text {(sc) }}^{\infty}}\left\|\nabla_{3} \omega\right\|_{\mathcal{L}_{\text {(sc) }}^{2}(H)} \lesssim \delta^{1 / 2} C, \\
& \left\|\left(\nabla_{3} \chi\right) Z\right\|_{\mathcal{L}_{(\mathrm{sc})}^{2}(H)} \lesssim \delta^{1 / 2}\|Z\|_{\mathcal{L}_{\text {(sc) }}^{\infty}}\left\|\nabla_{3} \chi\right\|_{\mathcal{L}_{(\mathrm{sc})}^{2}(H)} \lesssim C,
\end{aligned}
$$

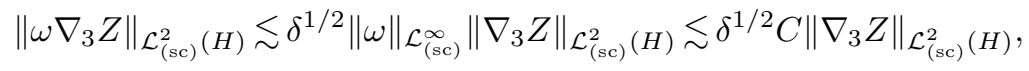

$$
\begin{aligned}
& \left\|\nabla_{3}(\varrho+\sigma)\right\|_{\mathcal{L}_{\text {(sc) }}^{2}(H)} \lesssim\|\nabla \underline{\beta}\|_{\mathcal{L}_{\text {(sc) }}^{2}(H)}+\left\|\left(\operatorname{tr} \underline{\chi}_{0}+\psi\right) \Psi\right\|_{\mathcal{L}_{\text {(sc) }}^{2}(H)} \lesssim \mathcal{R}_{1}+C, \\
& \left\|\Psi_{g} Z\right\|_{\mathcal{L}_{\text {(sc) }}^{2}(H)} \lesssim \delta^{1 / 2}\|Z\|_{\mathcal{L}_{\text {(sc) }}^{\infty}}\left\|\Psi_{g}\right\|_{\mathcal{L}_{(\text {sc) }}^{2}(H)} \lesssim \delta^{1 / 2} C, \\
& \left\|\Psi_{g} \underline{\chi}\right\|_{\mathcal{L}_{(\mathrm{sc})}^{2}(H)} \lesssim \delta^{1 / 2}\|\underline{\chi}\|_{\mathcal{L}_{(\mathrm{sc})}^{\infty}}\left\|\Psi_{g}\right\|_{\mathcal{L}_{\text {(sc) }}^{2}(H)} \lesssim C, \\
& \left\|\left(\nabla_{3} \psi_{34}\right) \psi\right\|_{\mathcal{L}_{(\mathrm{sc})}^{2}(H)} \lesssim \delta^{1 / 2}\|\psi\|_{\mathcal{L}_{(\mathrm{sc})}^{\infty}}\left\|\nabla_{3} \psi_{34}\right\|_{\mathcal{L}_{(\mathrm{sc})}^{2}(H)} \lesssim \delta^{1 / 2} C, \\
& \|\psi \psi Z\|_{\mathcal{L}_{\text {(sc) }}^{2}(H)} \lesssim \delta\|Z\|_{\mathcal{L}_{\text {(sc) }}^{\infty}}\|\psi\|_{\mathcal{L}_{(\mathrm{sc})}^{\infty}}\|\psi\|_{\mathcal{L}_{\text {(sc) }}^{2}(H)} \lesssim \delta^{1 / 2} C, \\
& \left\|\psi \psi_{34} \underline{\chi}\right\|_{\mathcal{L}_{(\mathrm{sc})}^{2}(H)} \lesssim \delta\|\underline{\chi}\|_{\mathcal{L}_{\text {(sc) }}^{\infty}}\|\psi\|_{\mathcal{L}_{\text {(sc) }}^{\infty}}\left\|\psi_{g}\right\|_{\mathcal{L}_{(\mathrm{sc})}^{2}(H)} \lesssim \delta^{1 / 2} C .
\end{aligned}
$$

\subsection{Estimates for $\left\|\nabla_{3} H\right\|_{\mathcal{L}_{\text {(sc) }}^{2}(H)}$}

The only quantity still requiring an estimate is $\left\|\nabla_{3} H\right\|_{\mathcal{L}_{(\mathrm{sc})}^{2}(H)}$. We use the relation $\left({ }^{23}\right)$

$$
\nabla_{3} H=\nabla_{3} \nabla O=\nabla \nabla_{3} O+\left[\nabla, \nabla_{3}\right] O=\nabla Z+(\nabla \underline{\chi}) O+\underline{\beta} O+\psi_{34} Z+\psi_{34} \underline{\chi} O .
$$

Therefore,

$$
\begin{aligned}
&\left\|\nabla_{3} H\right\|_{\mathcal{L}_{(\mathrm{sc})}^{2}(S)} \lesssim\|\nabla Z\|_{\mathcal{L}_{(\mathrm{sc})}^{2}(S)}+\|\nabla \underline{\chi}\|_{\mathcal{L}_{(\mathrm{sc})}^{2}(S)}+\left\|\Psi_{g}\right\|_{\mathcal{L}_{(\mathrm{sc})}^{2}(S)} \\
&+\delta^{1 / 2}\left\|\psi_{34}\right\|_{\mathcal{L}_{(\mathrm{sc})}^{2}(S)}\|Z\|_{\mathcal{L}_{\text {(sc) }}^{\infty}}+\delta^{1 / 2}\|\underline{\chi}\|_{\mathcal{L}_{(\mathrm{sc})}^{\infty}}\left\|\psi_{34}\right\|_{\mathcal{L}_{(\mathrm{sc})}^{2}(S)} \\
& \lesssim\|\nabla Z\|_{\mathcal{L}_{(\mathrm{sc})}^{2}(S)}+C .
\end{aligned}
$$
term.

$\left.{ }^{(22}\right)$ Note that $\Psi$ in the non-linear term may contain an $\underline{\alpha}$ component but not the anomalous $\alpha$

$\left({ }^{23}\right)$ Note the crucial cancellation of the anomalous term $\underline{\chi} H$. 
This immediately implies the bound

$$
\left\|\nabla_{3} H\right\|_{\mathcal{L}_{(\mathrm{sc})}^{2}(S)}+\left\|\nabla_{3} Z\right\|_{\mathcal{L}_{(\mathrm{sc})}^{2}(S)}+\left\|\nabla_{4} \nabla_{3} Z\right\|_{\mathcal{L}_{(\mathrm{sc})}^{2}(H)} \lesssim C
$$

A similar argument allows us to immediately strengthen the $\left\|\nabla_{3} H\right\|_{\mathcal{L}_{(\mathrm{sc})}^{2}(S)}$ estimate (unlike the one for $\nabla_{3} Z$ ) to the $\mathcal{L}_{(\mathrm{sc})}^{4}(S)$ norm:

$$
\left\|\nabla_{3} H\right\|_{\mathcal{L}_{(\mathrm{sc})}^{4}(S)} \leqslant C
$$

Furthermore,

$$
\begin{gathered}
\nabla_{4} \nabla_{3} H=\nabla_{4} \nabla Z+\left(\nabla_{4} \nabla \underline{\chi}\right) O+(\nabla \underline{\chi}) \chi O+\left(\nabla_{4} \underline{\beta}\right) O+\Psi_{g} \chi O+\left(\nabla_{4} \psi_{34}\right) Z \\
+\psi_{34} \nabla_{4} Z+\left(\nabla_{4} \psi_{34}\right) \underline{\chi} O+\psi_{34}\left(\nabla_{4} \underline{\chi}\right) O+\psi_{34} \underline{\chi} \chi O .
\end{gathered}
$$

We once again remind the reader of the possible anomalies for $\widehat{\chi}$ and $\underline{\hat{\chi}}$ in $\mathcal{L}_{(\mathrm{sc})}^{2}(S)$, double anomaly for $\operatorname{tr} \underline{\chi}$ in $\mathcal{L}_{(\mathrm{sc})}^{2}(S)$ and a simple anomaly in $\mathcal{L}_{(\mathrm{sc})}^{\infty}$, anomalies for $\nabla_{4} \widehat{\chi}$ and $\nabla_{3} \underline{\hat{\chi}}$. We estimate

$$
\begin{aligned}
& \left\|\nabla_{4} \nabla Z\right\|_{\mathcal{L}_{(\mathrm{sc})}^{2}(H)} \lesssim C, \\
& \left\|\nabla_{4} \nabla \underline{\chi}\right\|_{\mathcal{L}_{(\mathrm{sc})}^{2}(H)} \lesssim C, \\
& \|(\nabla \underline{\chi}) \chi\|_{\mathcal{L}_{(\mathrm{sc})}^{2}(H)} \lesssim \delta^{1 / 2}\|\chi\|_{\mathcal{L}_{(\mathrm{sc})}^{\infty}} \| \nabla \underline{\chi}_{\mathcal{L}_{(\mathrm{sc})}^{2}(H)} \lesssim \delta^{1 / 2} C, \\
& \left\|\nabla_{4} \underline{\beta}\right\|_{\mathcal{L}_{(\mathrm{sc})}^{2}(H)} \lesssim\left\|\nabla \Psi_{g}\right\|_{\mathcal{L}_{(\mathrm{sc})}^{2}(H)}+\left\|\psi \Psi_{g}\right\|_{\mathcal{L}_{(\mathrm{sc})}^{2}(H)} \lesssim \mathcal{R}_{1}+\delta^{1 / 2} C, \\
& \left\|\Psi_{g} \chi\right\|_{\mathcal{L}_{(\mathrm{sc})}^{2}(H)} \lesssim \delta^{1 / 2}\|\chi\|_{\mathcal{L}_{(\mathrm{sc})}^{\infty}}\left\|\Psi_{g}\right\|_{\mathrm{sc}^{2}(H)} \lesssim \delta^{1 / 2} C, \\
& \left\|\left(\nabla_{4} \psi_{34}\right) \underline{\chi}\right\|_{\mathcal{L}_{(\mathrm{sc})}^{2}(H)} \lesssim \delta^{1 / 2}\|\underline{\chi}\|_{\mathcal{L}_{(\mathrm{sc})}^{\infty}}\left\|\nabla_{4} \psi_{34}\right\|_{\mathcal{L}_{(\mathrm{sc})}^{2}(H)} \lesssim C, \\
& \left\|\psi_{34} \nabla_{4} \underline{\chi}\right\|_{\mathcal{L}_{(\mathrm{sc})}^{2}(H)} \lesssim \delta^{1 / 2}\|\psi\|_{\mathcal{L}_{(\mathrm{sc})}^{\infty}}\left\|\nabla_{4} \underline{\chi}\right\|_{\mathcal{L}_{(\mathrm{sc})}^{2}(H)} \lesssim \delta^{1 / 2} C, \\
& \left\|\psi_{34} \underline{\chi} \chi\right\|_{\mathcal{L}_{(\mathrm{sc})}^{2}(H)} \lesssim \delta\|\chi\|_{\mathcal{L}_{(\mathrm{sc})}^{\infty}}\|\underline{\chi}\|_{\mathcal{L}_{(\mathrm{sc})}^{\infty}}\left\|\psi_{34}\right\|_{\mathcal{L}_{(\mathrm{sc})}^{2}(H)} \lesssim \delta^{1 / 2} C .
\end{aligned}
$$

As a consequence we now established the following result.

Proposition 13.5. There exists a constant $C=C\left(\mathcal{O}_{[2]}, \mathcal{O}_{\infty}, \mathcal{R}_{[1]}, \underline{\mathcal{R}}_{[1]}\right)$ such that

$$
\left\|\nabla_{3} H\right\|_{\mathcal{L}_{(\mathrm{sc})}^{2}(S)}+\left\|\nabla_{3} Z\right\|_{\mathcal{L}_{(\mathrm{sc})}^{2}(S)}+\left\|\nabla_{4} \nabla_{3} Z\right\|_{\mathcal{L}_{(\mathrm{sc})}^{2}(H)}+\left\|\nabla_{4} \nabla_{3} H\right\|_{\mathcal{L}_{(\mathrm{sc})}^{2}(H)} \lesssim C .
$$




\subsection{Derivatives of the deformation tensor}

We now compute the derivatives of the deformation tensor $D \pi$ :

$$
\begin{array}{lll}
D_{4} \pi_{44}=0, & D_{4} \pi_{34}=-2\left(\nabla_{4}(\eta+\underline{\eta})\right) O-2(\eta+\underline{\eta}) \chi O, \\
D_{4} \pi_{33}=\frac{1}{4} \underline{\eta} Z, & D_{4} \pi_{3 a}=\frac{1}{2} \nabla_{4} Z+\underline{\eta}(\eta+\underline{\eta})-\frac{1}{2} \underline{\eta} H^{(s)}, \\
D_{4} \pi_{4 a}=0, & D_{4} \pi_{a b}=\nabla_{4} H^{s}, \\
D_{3} \pi_{44}=0, & D_{3} \pi_{34}=-2\left(\nabla_{3}(\eta+\underline{\eta})\right) O-2(\eta+\underline{\eta})(Z-\underline{\chi} O)-\frac{1}{4} \eta Z, \\
D_{3} \pi_{33}=0, & D_{3} \pi_{3 a}=\frac{1}{2} \nabla_{3} Z, \\
D_{3} \pi_{4 a}=-\eta(\eta+\underline{\eta})-\frac{1}{2} \eta H^{(s)}, & D_{3} \pi_{a b}=\nabla_{3} H^{(s)}+\frac{1}{4} \eta Z, \\
D_{c} \pi_{44}=0, & D_{c} \pi_{34}=-2(\nabla(\eta+\underline{\eta})) O-2(\eta+\underline{\eta}) H^{s}-\frac{1}{2} \chi Z, \\
D_{c} \pi_{33}=-\frac{1}{2} \underline{\chi} Z, & D_{c} \pi_{3 a}=\frac{1}{2} \nabla Z-\underline{\chi} H^{(s)}-2 \underline{\chi}(\eta+\underline{\eta}) O, \\
D_{c} \pi_{4 a}=-\chi H^{(s)}-2 \chi(\eta+\underline{\eta}) O, & D_{c} \pi_{a b}=\nabla H^{(s)}-\chi Z .
\end{array}
$$

Based on the results of the previous section, we easily deduce the following result.

Proposition 13.6. There exists a constant $C=C\left(\mathcal{O}_{[2]}, \mathcal{O}_{\infty}, \mathcal{R}_{[1]}, \underline{\mathcal{R}}_{[1]}\right)$ such that

$$
\|D \pi\|_{\mathcal{L}_{(\mathrm{sc})}^{2}(S)} \lesssim C \text {. }
$$

The only potentially problematic term is $\underline{\chi} H^{s}$, which can be estimated as

$$
\left\|\underline{\chi} H^{(s)}\right\|_{\mathcal{L}_{(\mathrm{sc})}^{2}(S)} \lesssim \delta^{1 / 2}\|\underline{\chi}\|_{\mathcal{L}_{(\mathrm{sc})}^{\infty}}^{\infty}\left\|H^{(s)}\right\|_{\mathcal{L}_{\text {(sc) }}^{2}} \lesssim C .
$$

It is precisely this term that requires a non-anomalous $\mathcal{L}_{(\mathrm{sc})}^{2}(S)$ estimate for $H^{(s)}$, which incidentally does not hold for the non-symmetrized derivative $H$.

\subsection{Theorem B}

We are now ready to state the main result of this section, mentioned in the introduction.

Theorem 13.7. (Theorem B) The deformation tensors ${ }^{(O)} \pi$ of the angular momentum operator $O$ satisfy the following estimate, with a constant $C=C\left(\mathcal{I}^{(0)}, \mathcal{R}, \underline{\mathcal{R}}\right)$ :

$$
\left\|^{(O)} \pi\right\|_{\mathcal{L}_{(\mathrm{sc})}^{4}(S)}+\left\|^{(O)} \pi\right\|_{\mathcal{L}_{(\mathrm{sc})}^{\infty}(S)} \lesssim C .
$$

Also all null components of the derivatives $D^{(O)} \pi$, with the exception of $\left(D_{3}{ }^{(O)} \pi\right)_{3 a}$, satisfy the estimate

$$
\left\|D^{(O)} \pi\right\|_{\mathcal{L}_{(\mathrm{sc})}^{4}(S)} \lesssim C
$$

Moreover,

$$
\left\|\left(D_{3}{ }^{(O)} \pi\right)_{3 a}-\nabla_{3} Z\right\|_{L^{4}(S)}+\left\|\sup _{\underline{u}}\left|\nabla_{3} Z\right|\right\|_{L^{2}(S)} \lesssim C .
$$




\section{Curvature estimates I}

In this and in all the remaining sections of the paper $C$ denotes a constant which depends on the initial data $\mathcal{I}_{0}$ and all the curvature norms $\mathcal{R}$ and $\underline{\mathcal{R}}$, including $\left\|\nabla_{4} \alpha\right\|_{\mathcal{L}_{(\mathrm{sc})}^{2}\left(H_{u}^{(0, \underline{u})}\right)}$ and $\left\|\nabla_{3} \alpha\right\|_{\mathcal{L}_{(\mathrm{sc})}^{2}\left(\underline{H}_{\underline{\underline{B}}}^{(0, u)}\right)}$. Using the results of the previous sections, we assume that the norms $\mathcal{O}$ of the Ricci coefficients are bounded by $C$.

\subsection{Preliminaries}

Let $W$ be a Weyl tensor field, with Hodge dual ${ }^{*} W$ satisfying the Bianchi equations with sources

$$
\operatorname{Div} W=J \text { and } \quad \operatorname{Div}^{*} W=J^{*},
$$

where $J$ and $J^{*}$ are Weyl currents, i.e.

$$
J_{[\alpha \beta \gamma]}=0, \quad J_{\alpha \beta \gamma}=-J_{\alpha \gamma \beta} \quad \text { and } \quad g^{\beta \gamma} J_{\beta \gamma \delta}=0
$$

and $J_{\alpha \beta \gamma}^{*}=\frac{1}{2} J_{\alpha \mu \nu} \in_{\beta \gamma}^{\mu \nu}$ is the right Hodge dual of $J$. Following the definitions of [CK], we let $Q[W]$ be the Bel-Robinson tensor of $W$. As proved there, we have the following result.

Proposition 14.1. Assume that $W$ satisfies (14.1). Given vector fields $X, Y$ and $Z$, and $P[W]=P[W](X, Y, Z)$ defined by $P[W]^{\alpha}:=Q[W]_{\alpha \beta \gamma \delta} X^{\beta} Y^{\gamma} Z^{\delta}$, we have

$\operatorname{Div} P[W]=\operatorname{Div} Q[W](X, Y, Z)+\frac{1}{2}(Q[W] \cdot \pi)(X, Y, Z)$,

where

$$
(Q[W] \cdot \pi)(X, Y, Z):=Q[W]\left({ }^{(X)} \pi, Y, Z\right)+Q[W]\left({ }^{(Y)} \pi, X, Z\right)+Q[W]\left({ }^{(Z)} \pi, X, Y\right)
$$

Thus, integrating on our fundamental domain $\mathcal{D}=\mathcal{D}(u, \underline{u})$,

$$
\begin{aligned}
\int_{H_{u}} Q[W](L, X, Y, Z)+\int_{\underline{H}_{\underline{u}}} Q[W](X, Y, Z, \underline{L}) \\
=\int_{H_{0}} Q[W](L, X, Y, Z)+\int_{\underline{H}_{0}} Q[W](X, Y, Z, \underline{L}) \\
\quad+\iint_{\mathcal{D}(u, \underline{u})} \operatorname{Div} Q[W](X, Y, Z)+\frac{1}{2} \iint_{\mathcal{D}(u, \underline{u})}(Q[W] \cdot \pi)(X, Y, Z) .
\end{aligned}
$$

In the particular case when $W$ is the curvature tensor $R$ (and thus $J=J^{*}=0$ ), recalling that the initial data on $\underline{\mathcal{H}}_{0}$ vanishes, we have the following result. 
Corollary 14.2. The following identity holds on our fundamental domain $\mathcal{D}(u, \underline{u})$ :

$$
\begin{aligned}
\int_{H_{u}} Q[R](L, X, Y, Z)+\int_{\underline{H}_{\underline{u}}} Q[R](X, Y, Z, \underline{L}) \\
=\int_{H_{0}} Q[R](L, X, Y, Z)+\frac{1}{2} \iint_{\mathcal{D}(u, \underline{u})}(Q[R] \cdot \pi)(X, Y, Z) .
\end{aligned}
$$

On the other hand, given a vector field $O$, we have

$$
\operatorname{Div}\left(\hat{\mathcal{L}}_{O} R\right)=J(O, R) \quad \text { and } \quad \operatorname{Div}\left({ }^{*} \hat{\mathcal{L}}_{O} R\right)=J^{*}(O, R)
$$

where $J(O, R)$ is a Weyl current (calculated below in Lemma 14.4) and $\hat{\mathcal{L}}_{O} R$ denotes the modified Lie derivative of the curvature tensor $R$, i.e. (following $[\mathrm{CK}]$ )

$$
\hat{\mathcal{L}}_{O} R=\mathcal{L}_{O} R-\frac{1}{8}\left(\operatorname{tr}^{(O)} \pi\right) R-\frac{1}{2}{ }^{(O)} \hat{\pi} R
$$

and

$$
\left({ }^{(O)} \hat{\pi} R\right)_{\alpha \beta \gamma \delta}={ }^{(O)} \hat{\pi}_{\alpha}^{\mu} W_{\mu \beta \gamma \delta}+{ }^{(O)} \hat{\pi}_{\beta}^{\mu} W_{\alpha \mu \gamma \delta}+{ }^{(O)} \hat{\pi}_{\gamma}^{\mu} W_{\alpha \beta \mu \delta}+{ }^{(O)} \hat{\pi}_{\delta}^{\mu} W_{\alpha \beta \gamma \mu},
$$

where ${ }^{(O)} \hat{\pi}$ is the traceless part of ${ }^{(O)} \pi$, that is ${ }^{(O)} \pi={ }^{(O)} \hat{\pi}+\frac{1}{4}\left(\operatorname{tr}^{(O)} \pi\right) g$. Observe that $\hat{\mathcal{L}}_{O} R$ is also a Weyl field and that the modified Lie derivative commutes with the Hodge dual, that is $\hat{\mathcal{L}}_{O}\left({ }^{*} R\right)={ }^{*} \hat{\mathcal{L}}_{O} R$. The following is a corollary of Proposition 14.1 and [CK, Proposition 7.1.1].

Corollary 14.3. Assume that $O$ is a vector field defined in our fundamental domain $\mathcal{D}(u, \underline{u})$, tangent to $\underline{\mathcal{H}}_{0}$. Then, with $H_{u}=H_{u}([0, \underline{u}])$,

$$
\begin{aligned}
& \int_{H_{u}} Q\left[\hat{\mathcal{L}}_{O} R\right](L, X, Y, Z)+\int_{\underline{H}_{\underline{u}}} Q\left[\hat{\mathcal{L}}_{O} R\right](X, Y, Z, \underline{L}) \\
& =\int_{H_{0}} Q\left[\hat{\mathcal{L}}_{O} R\right](L, X, Y, Z)+\frac{1}{2} \iint_{\mathcal{D}(u, \underline{u})}\left(Q\left[\hat{\mathcal{L}}_{O} R\right] \cdot \hat{\pi}\right)(X, Y, Z)+\iint_{\mathcal{D}(u, \underline{u})} D(R, O)(X, Y, Z),
\end{aligned}
$$

where $D(O, R):=\operatorname{Div} Q\left[\hat{\mathcal{L}}_{O} R\right]$ is given by the formula

$$
\begin{aligned}
D(O, R)_{\beta \gamma \delta}=\left(\hat{\mathcal{L}}_{O} R\right)_{\beta}{ }^{\mu}{ }_{\delta}{ }^{\nu} J(O, R)_{\mu \gamma \nu}+\left(\hat{\mathcal{L}}_{O} R\right)_{\beta}{ }^{\mu}{ }_{\gamma}{ }^{\nu} J(O, R)_{\mu \delta \nu} \\
+{ }^{*}\left(\hat{\mathcal{L}}_{O} R\right)_{\beta}{ }^{\mu}{ }_{\gamma}{ }^{\nu} J^{*}(O, R)_{\mu \delta \nu}+{ }^{*}\left(\hat{\mathcal{L}}_{O} R\right)_{\beta}{ }^{\mu}{ }_{\gamma}{ }^{\nu} J^{*}(O, R)_{\mu \delta \nu} .
\end{aligned}
$$

The Weyl current $J(O, R)$ is given by the following commutation formula, see [CK, Proposition 7.1.2]. 
LEMMA 14.4. We have

$$
\operatorname{Div}\left(\hat{\mathcal{L}}_{O} R\right)=J(O, R):=J^{1}(O, R)+J^{2}(O, R)+J^{3}(O, R),
$$

where

$$
\begin{aligned}
J^{1}(O, R)_{\beta \gamma \delta} & =\frac{1}{2}{ }^{(O)} \hat{\pi}^{\mu \nu} D_{\nu} R_{\mu \beta \gamma \delta}, \\
J^{2}(O, R)_{\beta \gamma \delta} & =\frac{1}{2}{ }^{(O)} p_{\lambda} R^{\lambda}{ }_{\beta \gamma \delta}, \\
J^{3}(O, R)_{\beta \gamma \delta} & =\frac{1}{2}\left({ }^{(O)} q_{\alpha \beta \lambda} R^{\alpha \lambda}{ }_{\gamma \delta}+{ }^{(O)} q_{\alpha \gamma \lambda} R^{\left.\alpha{ }_{\beta}{ }^{\lambda}{ }+{ }^{(O)} q_{\alpha \delta \lambda} R^{\alpha}{ }_{\beta \gamma}{ }^{\lambda}\right)}\right.
\end{aligned}
$$

and

$$
{ }^{(O)} p_{\gamma}=D^{\alpha}\left({ }^{(O)} \hat{\pi}_{\alpha \gamma}\right) \quad \text { and } \quad{ }^{(O)} q=D_{\beta}{ }^{(O)} \hat{\pi}_{\gamma \alpha}-D_{\gamma}{ }^{(O)} \hat{\pi}_{\beta \alpha}-\frac{1}{3}\left({ }^{(O)} p_{\gamma} g_{\alpha \beta}-{ }^{(O)} p_{\beta} g_{\alpha \gamma}\right) \text {. }
$$

In the remaining part of this section we should establish estimates for the norms $\mathcal{R}_{0}$ and $\underline{\mathcal{R}}_{0}$. We start with $\alpha$.

\subsection{Estimate for $\alpha$}

Applying Corollary 14.2 to $X=Y=Z=e_{4}$, we get

$$
\int_{H_{u}^{(0, \underline{u})}}|\alpha|^{2}+\int_{H_{\underline{u}}^{(0, u)}}|\beta|^{2} \lesssim \int_{H_{0}^{(0, \underline{u})}}|\alpha|^{2}+\int_{\mathcal{D}(u, \underline{u})}\left(Q[R] \cdot{ }^{(4)} \pi\right)\left(e_{4}, e_{4}, e_{4}\right) .
$$

Based on conservation of signature, we write schematically

$$
\left(Q[R] \cdot{ }^{(4)} \pi\right)\left(e_{4}, e_{4}, e_{4}\right)=\sum_{s_{1}+s_{2}+s_{3}=4} \phi^{\left(s_{1}\right)} \Psi^{\left(s_{2}\right)} \Psi^{\left(s_{3}\right)},
$$

with Ricci coefficients $\phi \in\{\chi, \omega, \eta, \underline{\omega}, \underline{\eta}\}$, null curvature components $\Psi$ and labels $s_{1}, s_{2}$ and $s_{3}$ denoting the signature of the corresponding component. In scale-invariant norms, we have

with

$$
\|\alpha\|_{\mathcal{L}_{(\mathrm{sc})}^{2}\left(H_{u}^{(0, \underline{u})}\right)}^{2}+\|\beta\|_{\mathcal{L}_{(\mathrm{sc})}^{2}\left(H_{\underline{u}}^{(0, u)}\right)}^{2} \lesssim\|\alpha\|_{\mathcal{L}_{(\mathrm{sc})}^{2}\left(H_{0}^{(0, \underline{u})}\right)}^{2}+I
$$

$$
I=\delta^{1 / 2} \sum_{s_{1}+s_{2}+s_{3}=4}\left\|\phi^{\left(s_{1}\right)}\right\|_{\mathcal{L}_{(\mathrm{sc})}^{\infty}} \int_{0}^{u}\left\|\Psi^{\left(s_{2}\right)}\right\|_{\mathcal{L}_{(\mathrm{sc})}^{2}\left(H_{u^{\prime}}^{(0, \underline{u})}\right)}\left\|\Psi^{\left(s_{3}\right)}\right\|_{\mathcal{L}_{(\mathrm{sc})}^{2}\left(H_{u^{\prime}}^{(0, \underline{u})}\right)} d u^{\prime} .
$$

By far the worst term occurs when $s_{2}=s_{3}=2$ and $s_{1}=0$. Observe also that, since the signature of a Ricci coefficient $\phi^{\left(s_{1}\right)}$ may not exceed $s_{1}=1$, neither $s_{2}$ nor $s_{3}$ can be zero, i.e. $\underline{\alpha}$ cannot occur among the curvature terms on the right. Using our estimates $\left\|\phi^{\left(s_{1}\right)}\right\|_{\mathcal{L}_{(\mathrm{sc})}^{\infty}} \lesssim C$, with $C=C\left(\mathcal{I}^{0}, \mathcal{R}, \underline{\mathcal{R}}\right)$, we deduce that

$$
\begin{aligned}
\|\alpha\|_{\mathcal{L}_{(\mathrm{sc})}^{2}\left(H_{u}^{(0, \underline{u})}\right)}^{2}+\|\beta\|_{\mathcal{L}_{(\mathrm{sc})}^{2}\left(H_{\underline{u}}^{(0, u)}\right)}^{2} \lesssim & \|\alpha\|_{\mathcal{L}_{(\mathrm{sc})}^{2}\left(H_{0}^{(0, \underline{u})}\right)}^{2}+C \delta^{1 / 2}\|\alpha\|_{\mathcal{L}_{(\mathrm{sc})}^{2}\left(H_{u}^{(0, \underline{u})}\right)}^{2} \\
& +C \mathcal{R}_{0} \delta^{1 / 2}\|\alpha\|_{\mathcal{L}_{(\mathrm{sc})}^{2}\left(H_{u}^{(0, \underline{u})}\right)}+C \delta^{1 / 2} \mathcal{R}_{0}^{2} .
\end{aligned}
$$

Therefore, recalling the anomalous character of $\mathcal{R}_{0}[\alpha]$ and $\underline{\mathcal{R}}_{0}[\beta]$, we deduce that

$$
\mathcal{R}_{0}[\alpha]+\underline{\mathcal{R}}_{0}[\beta] \lesssim \mathcal{I}^{0}+C \delta^{3 / 4} \mathcal{R}_{0} .
$$




\subsection{Remaining estimates}

We follow the procedure outlined in the introduction. Define the energy quantities

$$
\begin{aligned}
\mathcal{Q}_{0}(u, \underline{u})=\delta^{2} & \int_{\mathcal{H}_{u}^{(0, \underline{u})}} Q[R]\left(e_{4}, e_{4}, e_{4}, e_{4}\right)+\int_{\mathcal{H}_{u}^{(0, \underline{u})}} Q[R]\left(e_{3}, e_{4}, e_{4}, e_{4}\right) \\
& +\delta^{-1} \int_{\mathcal{H}_{u}^{(0, \underline{u})}} Q[R]\left(e_{3}, e_{3}, e_{4}, e_{4}\right)+\delta^{-2} \int_{\mathcal{H}_{u}^{(0, \underline{u})}} Q[R]\left(e_{3}, e_{3}, e_{3}, e_{4}\right)
\end{aligned}
$$

and

$$
\begin{aligned}
\underline{\mathcal{Q}}_{0}(u, \underline{u})=\delta^{2} & \int_{\underline{\mathcal{H}}_{\underline{u}}^{(0, u)}} Q[R]\left(e_{4}, e_{4}, e_{4}, e_{3}\right)+\int_{\underline{\mathcal{H}}_{\underline{u}}^{(0, u)}} Q[R]\left(e_{4}, e_{4}, e_{3}, e_{3}\right) \\
& +\delta^{-1} \int_{\underline{\mathcal{H}}_{\underline{u}}^{(0, u)}} Q[R]\left(e_{4}, e_{3}, e_{3}, e_{3}\right)+\delta^{-2} \int_{\underline{\mathcal{H}}_{\underline{u}}^{(0, u)}} Q[R]\left(e_{3}, e_{3}, e_{3}, e_{3}\right) .
\end{aligned}
$$

According to Corollary 14.2, for all possible choices of the vector fields $X, Y$ and $Z$ in the set $\left\{e_{4}, e_{3}\right\}$, we are led to the identity

$$
\mathcal{Q}_{0}(u, \underline{u})+\underline{\mathcal{Q}}_{0}(u, \underline{u}) \approx \mathcal{Q}_{0}(0, \underline{u})+\mathcal{E}_{0}(u, \underline{u}),
$$

where

$$
\begin{aligned}
\mathcal{E}_{0}(u, \underline{u})=\delta^{2} & \iint_{\mathcal{D}(u, \underline{u})} Q[R]\left({ }^{(4)} \pi, e_{4}, e_{4}\right)+\iint_{\mathcal{D}(u, \underline{u})} Q[R]\left({ }^{(4)} \pi, e_{3}, e_{4}\right) \\
& +\iint_{\mathcal{D}(u, \underline{u})} Q[R]\left({ }^{(3)} \pi, e_{4}, e_{4}\right)+\delta^{-1} \iint_{\mathcal{D}(u, \underline{u})} Q[R]\left({ }^{(4)} \pi, e_{3}, e_{3}\right) \\
& +\delta^{-1} \iint_{\mathcal{D}(u, \underline{u})} Q[R]\left({ }^{(3)} \pi, e_{4}, e_{3}\right)+\delta^{-2} \iint_{\mathcal{D}(u, \underline{u})} Q[R]\left({ }^{(3)} \pi, e_{3}, e_{3}\right),
\end{aligned}
$$

with ${ }^{(4)} \pi$ and ${ }^{(3)} \pi$ being the deformation tensors of $e_{4}$ and $e_{3}$, respectively. Every term appearing in the above integrands is linear in ${ }^{(4)} \pi$ or ${ }^{(3)} \pi$ and quadratic with respect to $R$. Also, all the components of ${ }^{(4)} \pi$ can be expressed in terms of our Ricci coefficients $\chi$, $\omega, \eta, \underline{\omega}$ and $\eta$. In fact one can easily check that

$$
\begin{aligned}
& { }^{(4)} \pi_{44}={ }^{(4)} \pi_{4 a}=0 \\
& { }^{(4)} \pi_{34}=g\left(D_{3} e_{4}, e_{4}\right)+g\left(D_{4} e_{4}, e_{3}\right)=4 \omega \\
& { }^{(4)} \pi_{33}=2 g\left(D_{3} e_{4}, e_{3}\right)=-8 \underline{\omega} \\
& { }^{(4)} \pi_{a b}=2 \chi_{a b} \\
& { }^{\left({ }^{4}\right)} \pi_{a 3}=g\left(D_{a} e_{4}, e_{3}\right)+g\left(D_{3} e_{4}, e_{a}\right)=2 \zeta_{a}+2 \eta_{a} .
\end{aligned}
$$

Similar formulas hold for ${ }^{(3)} \pi$, with $\chi$ replaced by $\chi$. Observe, in particular, that the term $\operatorname{tr} \underline{\chi}$ can only occur in connection with ${ }^{(3)} \pi$. Thus, all terms appearing in the $\mathcal{E}$ 
integrand are of the form $\phi \Psi_{1} \Psi_{2}$, where $\phi$ is one of the Ricci coefficients and $\Psi_{1}$ and $\Psi_{2}$ are null curvature components. Consider first the contribution to $\mathcal{Q}_{0}$ of the anomalous terms

$$
\delta^{2} \int_{\mathcal{H}_{u}^{(0, \underline{u})}} Q[R]\left(e_{4}, e_{4}, e_{4}, e_{4}\right)+\delta^{2} \int_{\underline{\mathcal{H}}_{\underline{u}}^{(0, u)}} Q[R]\left(e_{4}, e_{4}, e_{4}, e_{3}\right)
$$

obtained in (1.18) in the case when $X=Y=Z=e_{4}$. Since

$$
Q[R]\left(e_{4}, e_{4}, e_{4}, e_{4}\right)=|\alpha|^{2} \quad \text { and } \quad Q[R]\left(e_{4}, e_{4}, e_{4}, e_{3}\right)=|\beta|^{2},
$$

we derive that

$$
\begin{aligned}
\|\alpha\|_{L^{2}\left(H_{u}^{(0, \underline{u})}\right)}^{2}+\|\beta\|_{L^{2}\left(\underline{H}_{\underline{u}}^{(0, u)}\right)}^{2} & \approx\|\alpha\|_{L^{2}\left(H_{0}^{(0, \underline{u})}\right)}^{2}+\mathcal{E}_{01}(u, \underline{u}), \\
\mathcal{E}_{01}(u, \underline{u}) & \approx \iint_{\mathcal{D}(u, \underline{u})} Q\left({ }^{(4)} \pi, e_{4}, e_{4}\right) .
\end{aligned}
$$

As all the terms of the form $\phi \Psi_{1} \Psi_{2}$ have the same overall signature 4 . Thus, it is easy to derive the scale-invariant norm estimates

$$
\|\alpha\|_{\mathcal{L}_{(\mathrm{sc})}^{2}\left(H_{u}^{(0, \underline{u})}\right)}^{2}+\|\beta\|_{\mathcal{L}_{(\mathrm{sc})}^{2}\left(\underline{H}_{\underline{u}}^{(0, u)}\right)}^{2} \lesssim\|\alpha\|_{\mathcal{L}_{(\mathrm{sc})}^{2}\left(H_{0}^{(0, \underline{u})}\right)}^{2}+\mathcal{E}_{01}
$$

and

$$
\mathcal{E}_{01} \lesssim \delta^{1 / 2}\|\phi\|_{\mathcal{L}_{\text {(sc) }}^{\infty}} \int_{0}^{\underline{u}}\left\|\Psi_{1}\right\|_{\mathcal{L}_{(\mathrm{sc})}^{2}\left(H_{u}^{\left(0, \underline{u^{\prime}}\right)}\right)}\left\|\Psi_{2}\right\|_{\mathcal{L}_{(\mathrm{sc})}^{2}\left(H_{u}^{\left(0, \underline{u^{\prime}}\right)}\right)}
$$

The gain of $\delta^{1 / 2}$ is a reflection of the product estimates of type (2.19). Now, the only null curvature component which is anomalous with respect to the scale-invariant norms $\mathcal{L}_{\text {(sc) }}^{2}\left(H_{u}^{(0, \underline{u})}\right)$ is $\alpha$. On the other hand, the only Ricci coefficient which is anomalous in $\mathcal{L}_{(\mathrm{sc})}^{\infty}$ is $\operatorname{tr} \underline{\underline{\chi}}$. Indeed we have to decompose $\operatorname{tr} \underline{\chi}=\widetilde{\operatorname{tr} \underline{\chi}}+\operatorname{tr} \underline{\chi}_{0}$, where $\operatorname{tr} \underline{\chi}_{0}$ is the flat value of $\operatorname{tr} \underline{\chi}_{0}$ and therefore independent of $\delta$. This leads to a loss of $\delta^{1 / 2}$ in the corresponding estimates. Now, since $\operatorname{tr} \underline{\chi}$ cannot appear among the components of ${ }^{(4)} \pi$, we can lose at most a power of $\delta$ on the right-hand side of (14.6), which occurs only when $\Psi_{1}=\Psi_{2}=\alpha$. Fortunately the terms on the left of our integral inequality are also anomalous with

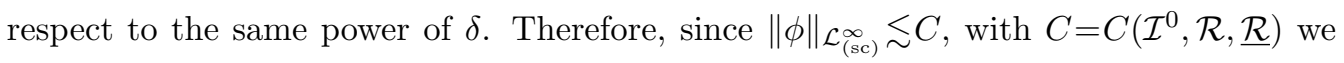
derive

$$
\mathcal{R}_{0}^{2}[\alpha]+\underline{\mathcal{R}}_{0}^{2}[\beta] \lesssim\left(\mathcal{I}^{(0)}\right)^{2}+\delta^{1 / 2} C \mathcal{R}_{0}^{2}
$$

Hence, for small $\delta>0$, we derive the bound

$$
\mathcal{R}_{0}[\alpha]+\underline{\mathcal{R}}_{0}[\beta] \lesssim \mathcal{I}^{(0)}+\delta^{1 / 4} C(\mathcal{R}, \underline{\mathcal{R}}),
$$


with $C$ being a universal constant depending only on the curvature norms $\mathcal{R}$ and $\underline{\mathcal{R}}$. We would like to show that all other error terms can be estimated in the same fashion, i.e. we would like to prove an estimate of the form

$$
\mathcal{R}_{0}+\underline{\mathcal{R}}_{0} \lesssim \mathcal{I}^{(0)}+\delta^{1 / 4} C(\mathcal{R}, \underline{\mathcal{R}})
$$

Assuming that a similar estimate holds for $\mathcal{R}_{1}+\underline{\mathcal{R}}_{1}$, we would thus conclude, for sufficiently small $\delta>0$, that

$$
\mathcal{R}+\underline{\mathcal{R}} \lesssim \mathcal{I}_{0}
$$

To prove (14.8), we observe that all remaining terms in (14.5) are scale invariant (i.e. they have the correct powers of $\delta$ ). In estimating the corresponding error terms, appearing on the right-hand side, we only have to be mindful of those which contain $\operatorname{tr} \underline{\chi}$ and $\alpha$. All other terms can be estimated by $\delta^{1 / 2} p(\mathcal{R}, \underline{\mathcal{R}})$ exactly as above. It is easy to check that all terms involving $\operatorname{tr} \underline{\chi}$ can only appear through ${ }^{(3)} \hat{\pi}_{34}$. Thus, it is easy to see that all such terms are of the form

$$
\begin{aligned}
& Q_{3444}{ }^{(3)} \hat{\pi}^{34} \approx-|\beta|^{2} \operatorname{tr} \underline{\chi}, \\
& Q_{3434}{ }^{(3)} \hat{\pi}^{34} \approx-\left(\varrho^{2}+\sigma^{2}\right) \operatorname{tr} \underline{\chi}, \\
& Q_{3433}{ }^{(3)} \hat{\pi}^{34}=-|\underline{\beta}|^{2} \operatorname{tr} \underline{\chi} .
\end{aligned}
$$

Hence, since $\operatorname{tr} \underline{\chi}=\widetilde{\operatorname{tr} \underline{\chi}}+\operatorname{tr} \underline{\chi}_{0}$, we easily deduce that all error terms containing $\operatorname{tr} \underline{\chi} \operatorname{can}$ be estimated by

$$
\delta^{-1} \int_{0}^{\underline{u}} \mathcal{Q}_{0}\left(u, \underline{u}^{\prime}\right) d \underline{u}^{\prime}+\delta^{1 / 2} C(\mathcal{R}, \underline{\mathcal{R}}) .
$$

It is easy to check that the integral term can be absorbed on the left by a Grönwall-type inequality. It thus remains to consider only the terms linear $\left({ }^{24}\right)$ in $\|\alpha\|_{\mathcal{L}_{(\mathrm{sc})}\left(H_{u}^{(0, \underline{u})}\right)}$, which we have already estimated above. These lead to error terms with no excess powers of $\delta$, which could be potentially dangerous. In fact we have to be a little more careful, because we would get an estimate of the form

$$
\mathcal{R}_{0}+\underline{\mathcal{R}}_{0} \lesssim \mathcal{I}^{(0)}+C(\mathcal{R}, \underline{\mathcal{R}})
$$

which is useless for large curvature norms $\mathcal{R}$ and $\underline{\mathcal{R}}$. To avoid this problem we need to refine our use of the ${ }^{(S)} \mathcal{O}_{0, \infty}$ norms. We observe that, among all the terms $\phi \Psi_{1} \Psi_{2}$ linear in $\alpha$, we can get better estimates for all, except those which contain a Ricci-coefficient

$\left({ }^{24}\right)$ By signature considerations there can be no terms quadratic in $\alpha$. 
component $\phi$ which is anomalous in $\mathcal{L}_{(\mathrm{sc})}^{4}(S)$. All other terms gain a power of $\delta^{1 / 4}$. Indeed the corresponding error terms in $\mathcal{E}_{1}$ can be estimated by $\left({ }^{25}\right)$

$$
\begin{aligned}
\delta^{1 / 2}\|\phi\|_{\mathcal{L}_{(\mathrm{sc})}^{4}(u, \underline{u})}\|\Psi\|_{\mathcal{L}_{(\mathrm{sc})}^{2}\left(H_{u}^{(0, \underline{u})}\right)}\|\nabla \alpha\|_{\mathcal{L}_{(\mathrm{sc})}\left(H_{u}^{(0, \underline{u})}\right)}^{1 / 2}\|\alpha\|_{\mathcal{L}_{(\mathrm{sc})}\left(H_{u}^{(0, \underline{u})}\right)}^{1 / 2} & \\
& \lesssim \delta^{1 / 4(S)} \mathcal{O}_{0,4} \mathcal{R}_{0} \mathcal{R}_{0}[\alpha]^{1 / 2} \mathcal{R}_{1}[\alpha]^{1 / 2}
\end{aligned}
$$

Denoting by $\mathcal{E}_{g}$ all such error terms, we thus have

$$
\left|\mathcal{E}_{g}\right| \lesssim \delta^{1 / 4} C(\mathcal{R}, \underline{\mathcal{R}})
$$

It remains to check the terms linear in $\alpha$ for which the Ricci-coefficient is anomalous in the $\mathcal{L}_{(\mathrm{sc})}^{4}$ norm, i.e. terms for which $\phi$ is either $\widehat{\chi}$ or $\underline{\hat{\chi}}$. It is easy to check that there are no terms linear in $\alpha$ which contain $\hat{\chi}$, and thus we only have to consider terms of the form $\underline{\hat{\chi}} \alpha \Psi$, which we denote by $\mathcal{E}_{b}$. As $\|\underline{\widehat{\chi}}\|_{\mathcal{L}_{(\mathrm{sc})}^{4}(u, \underline{u})}$ loses a power of $\delta^{1 / 4}$, we now have

$$
\begin{aligned}
\delta^{1 / 2}\|\underline{\hat{\chi}}\|_{\mathcal{L}_{(\mathrm{sc})}^{4}(u, \underline{u})}\|\Psi\|_{\mathcal{L}_{(\mathrm{sc})}^{2}\left(H_{u}^{(0, \underline{u})}\right)}\|\nabla \alpha\|_{\mathcal{L}_{(\mathrm{sc})}\left(H_{u}^{(0, \underline{u})}\right)}^{1 / 2}\|\alpha\|_{\mathcal{L}_{(\mathrm{sc})}\left(H_{u}^{(0, \underline{u})}\right)}^{1 / 2} & \\
& \lesssim{ }^{(S)} \mathcal{O}_{0,4}[\underline{\widehat{\chi}}] \mathcal{R}_{0} \mathcal{R}_{0}[\alpha]^{1 / 2} \mathcal{R}_{1}[\alpha]^{1 / 2} .
\end{aligned}
$$

Since we are left with no positive power of $\delta$, we must now be mindful of the fact that the estimates for ${ }^{(S)} \mathcal{O}_{0,4}$ depend at least linearly on the curvature norms $\mathcal{R}$ and $\underline{\mathcal{R}}$, in which case $\mathcal{E}_{b}$ is super-quadratic in $\mathcal{R}$ and $\underline{\mathcal{R}}$. We can however trace back the $\delta^{1 / 4}$ loss of $\|\underline{\hat{\chi}}\|_{\mathcal{L}_{(\mathrm{sc})}^{4}(u, \underline{u})}$ to initial data, i.e. upon a careful inspection we find (see estimate (2.12) of Theorem A)

$$
\|\underline{\widehat{\chi}}\|_{\mathcal{L}_{(\mathrm{sc})}^{4}(u, \underline{u})} \lesssim \delta^{-1 / 4} \mathcal{I}^{(0)}+C(\mathcal{R}, \underline{\mathcal{R}}) .
$$

Thus,

$$
\mathcal{E}_{b} \lesssim \mathcal{I}^{(0)} \mathcal{R}_{0} \mathcal{R}_{0}[\alpha]^{1 / 2} \mathcal{R}_{1}[\alpha]^{1 / 2}+\delta^{1 / 4} C(\mathcal{R}, \underline{\mathcal{R}})
$$

The above considerations lead us to conclude, back to (14.5), that

$$
\mathcal{R}_{0}+\underline{\mathcal{R}}_{0} \lesssim \mathcal{I}^{(0)}+c \mathcal{R}_{0}[\alpha]^{1 / 2} \mathcal{R}_{1}[\alpha]^{1 / 2}+\delta^{1 / 8} C(\mathcal{R}, \underline{\mathcal{R}})
$$

with a constant $c=c\left(\mathcal{I}^{(0)}\right)$ depending only on the initial data.

Remark. In the analysis above we have not considered the possibility that, among the terms in the integrands of $\mathcal{E}_{0}$, we can have terms of the form $\phi \Psi_{1} \Psi_{2}$ with at least one of the curvature terms being the null component $\underline{\alpha}$, which cannot be estimated along $H_{u}$. Among these terms, only those containing $\operatorname{tr} \underline{\chi}$ lead to terms which are $O(1)$ in $\delta$. These can be treated using $\underline{H}$, which leads to estimates of the form

$$
\mathcal{Q}_{0}(u, \underline{u})+\underline{\mathcal{Q}}_{0}(u, \underline{u}) \lesssim \mathcal{I}_{0}^{2}+\left(\int_{0}^{u} \mathcal{Q}_{0}\left(u^{\prime}, \underline{u}\right) d u^{\prime}+\delta^{-1} \int_{0}^{\underline{u}} \underline{\mathcal{Q}}_{0}\left(u, \underline{u}^{\prime}\right) d \underline{u}^{\prime}\right)+C \delta^{1 / 2},
$$

$\left({ }^{25}\right)$ This follows from the Gagliardo-Nirenberg inequality $\|\alpha\|_{L^{4}(u, \underline{u})}^{2} \lesssim\|\nabla \alpha\|_{L^{2}(u, \underline{u})}\|\alpha\|_{L^{2}(u, \underline{u})}$. 
with $C=C\left(\mathcal{I}^{(0)}, \mathcal{R}, \underline{\mathcal{R}}\right)$. The final estimate will follow from the lemma below (which can be easily proved by the method of continuity).

LEMma 14.5. Let $f(x, y)$ and $g(x, y)$ be positive functions defined in the rectangle $0 \leqslant x \leqslant x_{0}$ and $0 \leqslant y \leqslant y_{0}$ which satisfy the inequality

$$
f(x, y)+g(x, y) \lesssim J+a \int_{0}^{x} f\left(x^{\prime}, y\right) d x^{\prime}+b \int_{0}^{y} g\left(x, y^{\prime}\right) d y^{\prime}
$$

for some non-negative constants $a, b$ and $J$. Then, for all $0 \leqslant x \leqslant x_{0}$ and $0 \leqslant y \leqslant y_{0}$,

$$
f(x, y), g(x, y) \lesssim J e^{a x+b y}
$$

We summarize the results of this section in the following proposition.

Proposition 14.6. The following estimates hold with constants $C=C\left(\mathcal{I}^{(0)}, \mathcal{R}, \underline{\mathcal{R}}\right)$ and $c=c\left(\mathcal{I}^{(0)}\right)$, and a sufficiently small $\delta$ :

$$
\begin{aligned}
\mathcal{R}_{0}[\alpha]+\underline{\mathcal{R}}_{0}[\beta] & \lesssim \mathcal{I}^{(0)}+C \delta^{3 / 4}, \\
\mathcal{R}_{0}+\underline{\mathcal{R}}_{0} & \lesssim \mathcal{I}^{(0)}+c \mathcal{R}^{1 / 2}+\delta^{1 / 8} C .
\end{aligned}
$$

\section{Curvature estimates II}

We shall now estimate the first derivative of the null curvature components appearing in $\mathcal{R}_{1}$ and $\underline{\mathcal{R}}_{1}$. We apply (14.3) for the angular momentum vector fields $O$ as well as for the vector fields $L$ and $\underline{L}$. We prefer to work here with the vector fields $L$ and $\underline{L}$ instead of $e_{4}$ and $e_{3}$, as in the previous section, because their deformation tensors do not include $\omega$, respectively $\underline{\omega}$. This will make a difference in this section because we do not have good estimates for $\nabla_{4} \omega$ and $\nabla_{3} \underline{\omega}$ which would appear among the derivatives of ${ }^{(4)} \pi$ and ${ }^{(3)} \pi$. On the other hand, since $e_{3}$ and $e_{4}$ differ from $L$ and $\underline{L}$ only by the bounded factor $\Omega$, no other estimates will be affected.

\subsection{Deformation tensors of the vector fields $L$ and $\underline{L}$}

Below we list the components of ${ }^{L} \pi_{\alpha \beta}$ and ${ }^{L} \pi_{\alpha \beta}$ :

$$
\begin{array}{lll}
{ }^{L} \pi_{44}=0, & { }^{L} \pi_{43}=0, & { }^{L} \pi_{33}=-8 \Omega^{-1} \underline{\omega}, \\
{ }^{L} \pi_{4 a}=0, & { }^{L} \pi_{3 a}=\Omega^{-1}\left(\eta_{a}+\zeta_{a}\right)+\Omega^{-1} \nabla_{a} \log \Omega, & { }^{L} \pi_{a b}=\Omega^{-1} \chi_{a b}, \\
\underline{L}_{33}=0, & \underline{L}_{\pi_{43}}=0, & \underline{L}_{\pi_{44}}=-8 \Omega^{-1} \omega, \\
\underline{L}_{\pi_{3 a}}=0, & \underline{L}_{\pi_{4 a}}=\Omega^{-1}\left(\underline{\eta}_{a}+\zeta_{a}\right)+\Omega^{-1} \nabla_{a} \log \Omega, & \underline{L}_{\pi_{a b}}=\Omega^{-1} \underline{\chi}_{a b} .
\end{array}
$$

We start first with a sequence of lemmas. 


\subsection{Preliminaries}

Given a vector field $X$, we decompose both $\hat{\mathcal{L}}_{X} R$ and $D_{X} R$ into their null components $\alpha\left(\hat{\mathcal{L}}_{X} R\right), \beta\left(\hat{\mathcal{L}}_{X} R\right), \ldots, \underline{\alpha}\left(\hat{\mathcal{L}}_{X} R\right)$ and $\alpha\left(D_{X} R\right), \beta\left(D_{X} R\right), \ldots, \underline{\alpha}\left(D_{X} R\right)$. We consider these decompositions for the vector fields (note our discussion above concerning $X=L, \underline{L}$ and $\left.e_{a}, a=1,2\right)$. In the spirit of our discussion above, we write $e_{4}$ and $e_{3}$ instead of $L$ and $\underline{L}$, respectively. In the following lemma, we estimate the null components of $D_{X} R$, for $X=e_{3}, e_{4}, e_{a}$, in terms of $\mathcal{R}$ and $\underline{\mathcal{R}}$.

Lemma 15.1. Denoting the restrictions of the norms $\mathcal{R}$ and $\underline{\mathcal{R}}$ to the intervals $[0, u]$ and $[0, \underline{u}]$ by $\mathcal{R}_{u}$ and $\underline{\mathcal{R}}_{\underline{u}}$, respectively, we have, with $C=C\left(\mathcal{O}^{(0)}, \mathcal{R}, \underline{\mathcal{R}}\right)$, the anomalous estimate

$$
\delta^{1 / 2}\left\|\alpha\left(D_{3} R\right)\right\|_{\mathcal{L}_{(\mathrm{sc})}^{2}\left(H_{u}^{(0, \underline{u})}\right)}+\delta^{1 / 2}\left\|\beta\left(D_{a} R\right)\right\|_{\mathcal{L}_{(\mathrm{sc})}^{2}\left(H_{u}^{(0, \underline{u})}\right)} \lesssim \mathcal{I}^{(0)}+\delta^{1 / 4} C .
$$

We also have the regular estimates

$$
\begin{aligned}
& \left\|\alpha\left(D_{a} R\right)\right\|_{\mathcal{L}_{(\mathrm{sc})}^{2}\left(H_{u}^{(0, \underline{u})}\right)}+\left\|\beta\left(D_{3} R\right)\right\|_{\mathcal{L}_{\text {(sc) }}^{2}\left(H_{u}^{(0, \underline{u})}\right)}+\left\|\beta\left(D_{4} R\right)\right\|_{\mathcal{L}_{(\mathrm{sc})}^{2}\left(H_{u}^{(0, \underline{u})}\right)} \\
& \quad+\left\|(\varrho, \sigma)\left(D_{4} R\right)\right\|_{\mathcal{L}_{(\mathrm{sc})}^{2}\left(H_{u}^{(0, \underline{u})}\right)}+\left\|(\varrho, \sigma)\left(D_{3} R\right)\right\|_{\mathcal{L}_{(\mathrm{sc})}^{2}\left(H_{u}^{(0, \underline{u})}\right)}+\left\|(\varrho, \sigma)\left(D_{a} R\right)\right\|_{\mathcal{L}_{(\mathrm{sc})}^{2}\left(H_{u}^{(0, \underline{u})}\right)} \\
& \quad+\left\|\underline{\beta}\left(D_{4} R\right)\right\|_{\mathcal{L}_{(\mathrm{sc})}^{2}\left(H_{u}^{(0, \underline{u})}\right)}+\left\|\underline{\beta}\left(D_{a} R\right)\right\|_{\mathcal{L}_{(\mathrm{sc})}^{2}\left(H_{u}^{(0, \underline{u})}\right)}+\left\|\underline{\alpha}\left(D_{4} R\right)\right\|_{\mathcal{L}_{(\mathrm{sc})}^{2}\left(H_{u}^{(0, \underline{u})}\right)} \lesssim \mathcal{R}_{u}+\delta^{1 / 4} C
\end{aligned}
$$

and

$$
\begin{aligned}
& \left\|\beta\left(D_{3} R\right)\right\|_{\mathcal{L}_{(\mathrm{sc})}^{2}\left(H_{\underline{u}}^{(0, u)}\right)}+\left\|(\varrho, \sigma)\left(D_{4} R\right)\right\|_{\mathcal{L}_{(\mathrm{sc})}^{2}\left(H_{\underline{u}}^{(0, u)}\right)}+\left\|(\varrho, \sigma)\left(D_{3} R\right)\right\|_{\mathcal{L}_{(\mathrm{sc})}^{2}\left(H_{\underline{u}}^{(0, u)}\right)} \\
& +\left\|(\varrho, \sigma)\left(D_{4} R\right)\right\|_{\mathcal{L}_{(\mathrm{sc})}^{2}\left(H_{\underline{\underline{0}}}^{(0, u)}\right)}+\left\|\underline{\beta}\left(D_{4} R\right)\right\|_{\mathcal{L}_{\text {(sc) }}^{2}\left(H_{\underline{u}}^{(0, u)}\right)}+\left\|\underline{\beta}\left(D_{3} R\right)\right\|_{\mathcal{L}_{(\mathrm{sc})}^{2}\left(H_{\underline{u}}^{(0, u)}\right)} \\
& +\left\|\underline{\beta}\left(D_{a} R\right)\right\|_{\mathcal{L}_{(\mathrm{sc})}^{2}\left(H_{\underline{u}}^{(0, u)}\right)}+\left\|\underline{\alpha}\left(D_{4} R\right)\right\|_{\mathcal{L}_{(\mathrm{sc})}^{2}\left(H_{\underline{u}}^{(0, u)}\right)}+\left\|\underline{\alpha}\left(D_{a} R\right)\right\|_{\mathcal{L}_{(\mathrm{sc})}^{2}\left(H_{\underline{u}}^{(0, u)}\right)} \lesssim \underline{\mathcal{R}}_{\underline{u}}+\delta^{1 / 4} C .
\end{aligned}
$$

Remark 15.2. We note the special nature of the anomalies in $\alpha\left(D_{3} R\right)$ and $\beta\left(D_{a} R\right)$. Specifically, we can show that both terms can be written in the form $G+F$ with

$$
G=\left(\operatorname{tr} \underline{\chi}_{0}\right) \alpha
$$

and $F$ obeying the estimate

$$
\|F\|_{\mathcal{L}_{\text {(sc) }}^{2}\left(H_{\underline{u}}^{(0, u)}\right)}+\|F\|_{\mathcal{L}_{(\mathrm{sc})}^{2}\left(\underline{H}_{u}^{(0, \underline{u})}\right)} \leqslant C .
$$

Proof. Let $\Psi^{(s)}\left(D_{X} R\right)$ denote the null components of $D_{X} R$ and $\phi^{(s)}$ Ricci-coefficient components of signature $s$. Then, for $X \in\left\{L, e_{1}, e_{2}, \underline{L}\right\}$, recalling that $\operatorname{sgn}(X)=1, \frac{1}{2}, 0$ for $X=L, e_{a}, \underline{L}$, respectively, we write

$$
\Psi^{(s)}\left(D_{X} R\right)=\nabla_{X} \Psi^{(s)}+\sum_{s_{1}+s_{2}=s+\operatorname{sgn}(X)} \phi^{\left(s_{1}\right)} \Psi^{\left(s_{2}\right)} .
$$


Ignoring possible anomalies, we write

$$
\begin{aligned}
\left\|\Psi^{(s)}\left(D_{X} R\right)\right\|_{\mathcal{L}_{(\mathrm{sc})}^{2}\left(H_{u}^{(0, \underline{u})}\right)} & \lesssim\left\|\nabla_{X} \Psi^{(s)}(R)\right\|_{\mathcal{L}_{(\mathrm{sc})}^{2}\left(H_{u}^{(0, \underline{u})}\right)}+\delta^{1 / 2(S)} \mathcal{O}_{0, \infty} \mathcal{R}_{0} \\
& \lesssim\left\|\nabla_{X} \Psi^{(s)}(R)\right\|_{\mathcal{L}_{(\mathrm{sc})}^{2}\left(H_{u}^{(0, \underline{u})}\right)}+C \delta^{1 / 2} \\
\left\|\Psi^{(s)}\left(D_{X} R\right)\right\|_{\mathcal{L}_{(\mathrm{sc})}^{2}\left(\underline{H}_{\underline{u}}^{(0, u)}\right)} & \lesssim\left\|\nabla_{X} \Psi^{(s)}(R)\right\|_{\mathcal{L}_{(\mathrm{sc})}^{2}\left(\underline{H}_{\underline{u}}^{(0, u)}\right)}+\delta^{1 / 2(S)} \mathcal{O}_{0, \infty} \underline{\mathcal{R}}_{0} \\
& \lesssim\left\|\nabla_{X} \Psi^{(s)}(R)\right\|_{\mathcal{L}_{(\mathrm{sc})}^{2}\left(\underline{H}_{\underline{u}}^{(0, u)}\right)}+C \delta^{1 / 2} .
\end{aligned}
$$

We only have to pay special attention to the case when $\phi^{\left(s_{1}\right)}=\operatorname{tr} \underline{\chi}$ and $\Psi^{\left(s_{2}\right)}=\alpha$. If $s_{2}=2$, i.e. $\Psi^{\left(s_{2}\right)}=\alpha$, then $s_{1}$ can be $1, \frac{1}{2}$ and 0 . The case $s_{1}=1$ occurs only if $X=e_{4}$, which is not covered by the lemma. The case $s_{2}=2$ and $s_{1}=\frac{1}{2}$ is regular. Indeed, in that case $s+\operatorname{sgn}(X)=\frac{5}{2}$. Thus, either $s=2$ and $X=e_{a}$, or $s=\frac{3}{2}$ and $X=L$. In both cases, we simply estimate the worst quadratic term, on the right-hand side of (15.1), with $s_{2}=2$, by

$$
\begin{aligned}
\|\phi \alpha\|_{\mathcal{L}_{(\mathrm{sc})}^{2}\left(H_{u}^{(0, \underline{u})}\right)} & \lesssim \delta^{1 / 2}\|\phi\|_{\mathcal{L}_{(\mathrm{sc})}^{4}\left(H_{u}^{(0, \underline{u})}\right)}\|\alpha\|_{\mathcal{L}_{(\mathrm{sc})}^{4}\left(H_{u}^{(0, \underline{u})}\right)} \\
& \lesssim \delta^{1 / 2(S)} \mathcal{O}_{0,4}[\phi]\|\alpha\|_{\mathcal{L}_{(\mathrm{sc})}^{2}\left(H_{u}^{(0, \underline{u})}\right)}\|\nabla \alpha\|_{\mathcal{L}_{(\mathrm{sc})}^{2}\left(H_{u}^{(0, \underline{u})}\right)}^{1 / 2} \\
& \lesssim \delta^{1 / 4(S)} \mathcal{O}_{0,4}[\phi] \mathcal{R}_{0}[\alpha]^{1 / 2} \mathcal{R}_{1}[\alpha]^{1 / 2} \lesssim C \delta^{1 / 4}
\end{aligned}
$$

The principal term is either $\nabla \alpha$ in the first case or $\nabla_{L} \beta$ in the second. In the second situation, using the null Bianchi identities (proceeding as above with the term of the form $\phi \alpha$ ), we have

$$
\left\|\nabla_{L} \beta\right\|_{\mathcal{L}_{(\mathrm{sc})}^{2}\left(H_{u}^{(0, \underline{u})}\right)} \lesssim\|\nabla \alpha\|_{\mathcal{L}_{(\mathrm{sc})}^{2}\left(H_{u}^{(0, \underline{u})}\right)}+C \delta^{1 / 4}
$$

In the case $s_{2}=2$ and $s_{1}=0, \operatorname{tr} \underline{\chi}$ can appear among the quadratic terms on the right. In that case $s+\operatorname{sgn}(X)=2$. The case $s=2$ and $X=\underline{L}$ corresponds to the anomalous estimate for $\alpha\left(D_{\underline{L}} R\right)$. In that case the estimate is

$$
\left\|\alpha\left(D_{\underline{L}} R\right)\right\|_{\mathcal{L}_{(\mathrm{sc})}^{2}\left(H_{u}^{(0, \underline{u})}\right)} \lesssim\left\|\nabla_{\underline{L}} \alpha\right\|_{\mathcal{L}_{(\mathrm{sc})}^{2}\left(H_{u}^{(0, \underline{u})}\right)}+\left(1+\delta^{1 / 2} C\right)\|\alpha\|_{\mathcal{L}_{(\mathrm{sc})}^{2}\left(H_{u}^{(0, \underline{u})}\right)}+\delta^{1 / 2} C .
$$

Also, in view of the Bianchi identities (3.7),

$$
\left\|\nabla_{\underline{L}} \alpha\right\|_{\mathcal{L}_{(\mathrm{sc})}^{2}\left(H_{u}^{(0, \underline{u})}\right)} \lesssim\|\nabla \beta\|_{\mathcal{L}_{(\mathrm{sc})}^{2}\left(H_{u}^{(0, \underline{u})}\right)}+\|\alpha\|_{\mathcal{L}_{(\mathrm{sc})}^{2}\left(H_{u}^{(0, \underline{u})}\right)}+C \delta^{1 / 2} .
$$

Hence, in view of our estimate for $\alpha$ in the previous section,

$$
\begin{aligned}
\delta^{1 / 2}\left\|\alpha\left(D_{L} R\right)\right\|_{\mathcal{L}_{(\mathrm{sc})}^{2}\left(H_{u}^{(0, \underline{u})}\right)} & \lesssim \delta^{1 / 2}\left\|\nabla_{L} \alpha\right\|_{\mathcal{L}_{(\mathrm{sc})}^{2}\left(H_{u}^{(0, \underline{u})}\right)}+\left(1+\delta^{1 / 2} C\right) \delta^{1 / 2}\|\alpha\|_{\mathcal{L}_{(\mathrm{sc})}^{2}\left(H_{u}^{(0, \underline{u})}\right)} \\
& \lesssim \mathcal{I}^{(0)}+\delta^{1 / 4} C
\end{aligned}
$$


as desired. We also need to consider the case $s_{2}=2, s_{1}=0, s=\frac{3}{2}$ and $X=e_{a}$. Then, due to the term $\left(\operatorname{tr} \underline{\chi}_{0}\right) \alpha$ on the right-hand side of (15.1), we have

$$
\left\|\beta\left(D_{a} R\right)\right\|_{\mathcal{L}_{(\mathrm{sc})}^{2}\left(H_{u}^{(0, \underline{u})}\right)} \lesssim\|\nabla \beta\|_{\mathcal{L}_{(\mathrm{sc})}^{2}\left(H_{u}^{(0, \underline{u})}\right)}+\|\alpha\|_{\mathcal{L}_{(\mathrm{sc})}^{2}\left(H_{u}^{(0, \underline{u})}\right)}+C \delta^{1 / 4}
$$

Thus,

$$
\delta^{1 / 2}\left\|\beta\left(D_{a} R\right)\right\|_{\mathcal{L}_{(\mathrm{sc})}^{2}\left(H_{u}^{(0, \underline{u})}\right)} \lesssim \mathcal{I}^{(0)}+C \delta^{1 / 4}
$$

which is the second anomalous estimate.

It remains to consider the case when $s_{2}<2$ and $s_{1}=0$. In the worst case, when a quadratic term on the right-hand side of (15.1) is of the form $\left(\operatorname{tr} \underline{\chi}_{0}\right) \Psi^{\left(s_{2}\right)}$, we make the following correction to estimate (15.2):

$$
\begin{aligned}
&\left\|\Psi^{(s)}\left(D_{X} R\right)\right\|_{\mathcal{L}_{(\mathrm{sc})}^{2}\left(H_{u}^{(0, \underline{u})}\right)} \lesssim\left\|\nabla_{X} \Psi^{(s)}(R)\right\|_{\mathcal{L}_{(\mathrm{sc})}^{2}\left(H_{u}^{(0, \underline{u})}\right)}+\left\|\Psi^{\left(s_{2}\right)}\right\|_{\mathcal{L}_{(\mathrm{sc})}^{2}\left(H_{u}^{(0, \underline{u})}\right)}+C \delta^{1 / 4} \\
& \lesssim\left\|\nabla_{X} \Psi^{(s)}(R)\right\|_{\mathcal{L}_{(\mathrm{sc})}^{2}\left(H_{u}^{(0, \underline{u})}\right)}+\mathcal{R}_{u}+C \delta^{1 / 4}, \\
&\left\|\Psi^{(s)}\left(D_{X} R\right)\right\|_{\mathcal{L}_{(\mathrm{sc})}^{2}\left(\underline{H}_{\underline{u}}^{(0, u)}\right)} \lesssim\left\|\nabla_{X} \Psi^{(s)}(R)\right\|_{\mathcal{L}_{(\mathrm{sc})}^{2}\left(\underline{H}_{\underline{u}}^{(0, u)}\right)}+\left\|\Psi^{\left(s_{2}\right)}\right\|_{\mathcal{L}_{(\mathrm{sc})}^{2}\left(\underline{H}_{\underline{u}}^{(0, u)}\right)}+C \delta^{1 / 4} \\
& \lesssim\left\|\nabla_{X} \Psi^{(s)}(R)\right\|_{\mathcal{L}_{(\mathrm{sc})}^{2}\left(\underline{H}_{\underline{u}}^{(0, u)}\right)}+\underline{\mathcal{R}}_{\underline{u}}+C \delta^{1 / 4} .
\end{aligned}
$$

These imply the regular estimates of the lemma for the case $X=e_{a}$. For the case when $X \in\{L, \underline{L}\}$, we can express $\nabla_{X} \Psi^{(s)}(R)$ using the Bianchi identities

$$
\begin{array}{ll}
\nabla_{3} \Psi^{(s)}=\nabla \Psi^{(s-1 / 2)}+\sum_{s_{1}+s_{2}=s} \phi^{\left(s_{1}\right)} \Psi^{\left(s_{2}\right)}, & 0<s<2, \\
\nabla_{4} \Psi^{(s)}=\nabla \Psi^{(s+1 / 2)}+\sum_{s_{1}+s_{2}=s+1} \phi^{\left(s_{1}\right)} \Psi^{\left(s_{2}\right)}, & 0 \leqslant s<2 .
\end{array}
$$

The worst quadratic terms which can appear on the right are of the form $(\operatorname{tr} \chi) \Psi^{(s)}$ with $s<2$, which can be easily estimated. We thus derive all the regular estimates of the lemma.

LEMMA 15.3. The following estimates for the Lie derivatives $\hat{\mathcal{L}}_{X} R$, with respect to $X \in\{\underline{L}, L, O\}$, hold true:

$$
\begin{aligned}
&\left\|\alpha\left(\hat{\mathcal{L}}_{L} R\right)-\nabla_{L} \alpha\right\|_{\mathcal{L}_{(\mathrm{sc})}^{2}\left(H_{u}^{(0, \underline{u})}\right)} \lesssim C \\
& \delta^{1 / 2}\left\|\alpha\left(\hat{\mathcal{L}}_{\underline{L}} R\right)-\nabla_{\underline{L}} \alpha\right\|_{\mathcal{L}_{(\mathrm{sc})}^{2}\left(H_{u}^{(0, \underline{u})}\right)} \lesssim \mathcal{R}_{0}+C \delta^{3 / 4} .
\end{aligned}
$$


Also,

$$
\begin{array}{rlr}
\left\|\Psi^{(s)}\left(\hat{\mathcal{L}}_{L} R\right)-\left(\nabla_{L} \Psi\right)^{(s)}\right\|_{\mathcal{L}_{(\mathrm{sc})}^{2}\left(H_{u}^{(0, \underline{u})}\right)} \lesssim C \delta^{1 / 4}, & 1 \leqslant s \leqslant \frac{5}{2}, \\
\left\|\Psi^{(s)}\left(\hat{\mathcal{L}}_{\underline{L}} R\right)-\left(\nabla_{\underline{L}} \Psi\right)^{(s)}\right\|_{\mathcal{L}_{(\mathrm{sc})}^{2}\left(H_{u}^{(0, \underline{u})}\right)} \lesssim \mathcal{R}_{0}+C \delta^{1 / 4}, & 1 \leqslant s \leqslant \frac{3}{2}, \\
\left\|\Psi^{(s)}\left(\hat{\mathcal{L}}_{\underline{L}} R\right)-\left(\nabla_{\underline{L}} \Psi\right)^{(s)}\right\|_{\mathcal{L}_{(\mathrm{sc})}^{2}\left(\underline{H}_{\underline{u}}^{(0, u)}\right)} \lesssim \mathcal{R}_{0}+C \delta^{1 / 4}, & s \leqslant \frac{1}{2} .
\end{array}
$$

For $X=O$ we have the estimates

$$
\begin{aligned}
& \left\|\Psi^{(s)}\left(\hat{\mathcal{L}}_{O} R\right)-\left(\nabla_{O} \Psi\right)^{(s)}\right\|_{\mathcal{L}_{(\mathrm{sc})}^{2}\left(H_{u}^{(0, \underline{u})}\right)} \lesssim C \delta^{1 / 4}, \quad 1 \leqslant s \leqslant \frac{5}{2}, \\
& \left\|\Psi^{(s)}\left(\hat{\mathcal{L}}_{O} R\right)-\left(\nabla_{O} \Psi\right)^{(s)}\right\|_{\mathcal{L}_{(\mathrm{sc})}^{2}\left(\underline{H}_{\underline{u}}^{(0, u)}\right)} \lesssim C \delta^{1 / 4}, \quad \frac{1}{2} \leqslant s \leqslant 2 .
\end{aligned}
$$

Proof. We will make use of the regular $\mathcal{L}_{(\mathrm{sc})}^{\infty}$ estimates for Ricci coefficients

$$
\phi \in\{\chi, \omega, \eta, \underline{\widehat{\chi}}, \widetilde{\operatorname{tr}} \underline{\chi}, \underline{\omega}, \underline{\eta}\} .
$$

We also make use of the following estimates for $\nabla O$ and ${ }^{(O)} \pi$.

We write, recalling the definition of the Lie derivative and with $E$ denoting the set $\left\{e_{1}, e_{2}, e_{3}, e_{4}\right\}$,

$$
\begin{aligned}
\Psi^{(s)}\left(\mathcal{L}_{X} R\right) & =X\left(\Psi^{(s)}\right)-\sum_{s_{1}+s_{2}=s} \sum_{Y \in E}([X, Y])^{\left(s_{1}\right)} \Psi^{\left(s_{2}\right)} \\
& =\mathcal{A}_{X}\left(\Psi^{(s)}\right)-\sum_{s_{1}+s_{2}=s} \sum_{Y \in E}\left(([X, Y])^{\left(s_{1}\right)}\right)^{\perp} \Psi^{\left(s_{2}\right)} .
\end{aligned}
$$

Here $\mathcal{L}_{X}\left(\Psi^{(s)}\right)$ denotes the projection of the Lie derivative on the surface $S(u, \underline{u})$ and $[X, Y]^{\perp}$ the orthogonal component of $[X, Y]$, i.e.

$$
[X, Y]^{\perp}=-\frac{1}{2} g\left([X, Y], e_{3}\right) e_{4}-\frac{1}{2} g\left([X, Y], e_{4}\right) e_{3} .
$$

Consider first the case when $X \in\{L, \underline{L}\}$. In that case $[X, Y]^{\perp}$ depends only on the regular Ricci coefficients $\omega, \eta, \underline{\omega}$ and $\underline{\eta}$. Therefore, taking into account the worst possible case when $\alpha$ appear among the quadratic terms (in which case we appeal to $\mathcal{L}_{(\mathrm{sc})}^{4}$ estimates), we derive

$$
\begin{aligned}
& \left\|\Psi^{(s)}\left(\mathcal{L}_{L} R\right)-\left(\mathcal{L}_{L} \Psi\right)^{(s)}\right\|_{\mathcal{L}_{(\mathrm{sc})}^{2}\left(H_{u}^{(0, \underline{u})}\right)} \lesssim C \delta^{1 / 4}, \quad 1 \leqslant s \leqslant 3, \\
& \left\|\Psi^{(s)}\left(\mathcal{L}_{\underline{L}} R\right)-\left(\mathcal{L}_{\underline{L}} \Psi\right)^{(s)}\right\|_{\mathcal{L}_{(\mathrm{sc})}^{2}\left(H_{u}^{(0, \underline{u})}\right)} \lesssim C \delta^{1 / 4}, \quad 1 \leqslant s \leqslant 2, \\
& \left\|\Psi^{(s)}\left(\mathcal{L}_{\underline{L}} R\right)-\left(\mathcal{L}_{\underline{L}} \Psi\right)^{(s)}\right\|_{\mathcal{L}_{(\mathrm{sc})}^{2}\left(\underline{H}_{\underline{u}}^{(0, u)}\right)} \lesssim C \delta^{1 / 4}, \quad 0 \leqslant s \leqslant \frac{1}{2} .
\end{aligned}
$$

On the other hand, schematically,

$$
\mathcal{A}_{L} \Psi^{(s)}=\nabla_{L} \Psi^{(s)}+\sum_{s_{1}+s_{2}=1+s} \phi^{\left(s_{1}\right)} \Psi^{\left(s_{2}\right)},
$$


with $\phi^{\left(s_{1}\right)} \in\{\chi, \eta, \underline{\eta}\}$. In the particular case $s=3$, we can have a double anomaly of the form $\chi \alpha$. In that case,

$$
\left\|\mathcal{L}_{L} \alpha-\nabla_{L} \alpha\right\|_{\mathcal{L}_{(\mathrm{sc})}^{2}\left(H_{u}^{(0, \underline{u})}\right)} \lesssim C \delta^{1 / 2}\|\alpha\|_{\mathcal{L}_{(\mathrm{sc})}^{2}\left(H_{u}^{(0, \underline{u})}\right)}+C \delta^{1 / 2} .
$$

Therefore, $\left\|\mathcal{L}_{L} \alpha-\nabla_{L} \alpha\right\|_{\mathcal{L}_{(\mathrm{sc})}^{2}\left(H_{u}^{(0, \underline{u})}\right)} \lesssim C$, from which, combining with (15.9),

$$
\left\|\alpha\left(\mathcal{L}_{L} R\right)-\nabla_{L} \alpha\right\|_{\mathcal{L}_{(\mathrm{sc})}^{2}\left(H_{u}^{(0, \underline{u})}\right)} \lesssim C .
$$

Recalling the definition of $\hat{\mathcal{L}}_{L} R$, we deduce that

$$
\left\|\alpha\left(\hat{\mathcal{L}}_{L} R\right)-\nabla_{L} \alpha\right\|_{\mathcal{L}_{(\mathrm{sc})}^{2}\left(H_{u}^{(0, \underline{u})}\right)} \lesssim C,
$$

as desired.

We now consider all other cases, $1 \leqslant s \leqslant \frac{5}{2}$. Since there are no double anomalies, we deduce (using $\mathcal{L}_{(\mathrm{sc})}^{4}(S)$ estimates for the term containing $\alpha$ ) that

$$
\left\|\mathcal{A}_{L} \Psi^{(s)}-\left(\nabla_{L} \Psi\right)^{(s)}\right\|_{\mathcal{L}_{(\mathrm{sc})}^{2}\left(H_{u}^{(0, \underline{u})}\right)} \lesssim C \delta^{1 / 4} .
$$

Hence, combining with (15.9),

$$
\left\|\Psi^{(s)}\left(\mathcal{L}_{L} R\right)-\left(\nabla_{L} \Psi\right)^{(s)}\right\|_{\mathcal{L}_{\text {(sc) }}^{2}\left(H_{u}^{(0, \underline{u})}\right)} \lesssim C \delta^{1 / 4} .
$$

Recalling the definition of $\Psi^{(s)}\left(\mathcal{L}_{L} R\right)$, we deduce that

$$
\left\|\Psi^{(s)}\left(\hat{\mathcal{L}}_{L} R\right)-\left(\nabla_{L} \Psi\right)^{(s)}\right\|_{\mathcal{L}_{(\mathrm{sc})}^{2}\left(H_{u}^{(0, \underline{u})}\right)} \lesssim C \delta^{1 / 4}, \quad 1 \leqslant s \leqslant \frac{5}{2},
$$

as desired.

We now consider the estimates for $\underline{L}$. We have

$$
\mathcal{A}_{\underline{L}} \Psi^{(s)}=\nabla_{\underline{L}} \Psi^{(s)}+\left(\operatorname{tr} \underline{\chi}_{0}\right) \Psi^{(s)}+\sum_{s_{1}+s_{2}=s} \phi^{\left(s_{1}\right)} \Psi^{\left(s_{2}\right)}
$$

with $\phi^{\left(s_{1}\right)} \in\{\eta, \widehat{\chi}, \widetilde{\operatorname{tr}} \underline{\chi}, \underline{\eta}\}$. Observe that the worst terms $\left(\operatorname{tr} \underline{\chi}_{0}\right) \alpha$ can only appear for $s=2$. In that case,

$$
\left\|\mathcal{L}_{\underline{L}} \alpha-\nabla_{\underline{L}} \alpha\right\|_{\mathcal{L}_{(\mathrm{sc})}^{2}\left(H_{u}^{(0, \underline{u})}\right)} \lesssim\|\alpha\|_{\mathcal{L}_{(\mathrm{sc})}^{2}\left(H_{u}^{(0, \underline{u})}\right)}+C \delta^{1 / 4} \lesssim \delta^{-1 / 2} \mathcal{R}_{0}+C \delta^{1 / 4}
$$

Thus, combining with (15.9),

$$
\delta^{1 / 2}\left\|\alpha\left(\mathcal{L}_{\underline{L}} R\right)-\nabla_{\underline{L}} \alpha\right\|_{\mathcal{L}_{(\mathrm{sc})}^{2}\left(H_{u}^{(0, \underline{u})}\right)} \lesssim \mathcal{R}_{0}+C \delta^{3 / 4}
$$


Finally, recalling the definition of $\alpha\left(\hat{\mathcal{L}}_{\underline{L}} R\right)$, we deduce that

$$
\delta^{1 / 2}\left\|\alpha\left(\hat{\mathcal{L}}_{\underline{L}} R\right)-\nabla_{\underline{L}} \alpha\right\|_{\mathcal{L}_{(\mathrm{sc})}^{2}\left(H_{u}^{(0, \underline{u})}\right)} \lesssim \mathcal{R}_{0}+C \delta^{3 / 4}
$$

as desired.

In all other cases, $1 \leqslant s \leqslant \frac{3}{2}$, we have

$$
\left\|\mathcal{A}_{\underline{L}} \Psi^{(s)}-\left(\nabla_{\underline{L}} \Psi\right)^{(s)}\right\|_{\mathcal{L}_{(\mathrm{sc})}^{2}\left(H_{u}^{(0, \underline{u})}\right)} \lesssim\left\|\Psi^{(s)}\right\|_{\mathcal{L}_{(\mathrm{sc})}^{2}\left(H_{u}^{(0, \underline{u})}\right)}+C \delta^{1 / 4} \lesssim \mathcal{R}_{0}+C \delta^{1 / 4} .
$$

Hence, combining with (15.9) and recalling the definition of $\hat{\mathcal{L}}$, we deduce that

$$
\left\|\Psi^{(s)}\left(\hat{\mathcal{L}}_{\underline{L}} R\right)-\left(\nabla_{\underline{L}} \Psi\right)^{(s)}\right\|_{\mathcal{L}_{(\mathrm{sc})}^{2}\left(H_{u}^{(0, \underline{u})}\right)} \lesssim \mathcal{R}_{0}+C \delta^{1 / 4}
$$

as desired.

We now consider the case when $X=O$. In view of (15.8),

$$
\left\|\Psi^{(s)}\left(\mathcal{L}_{O} R\right)-\left(\mathcal{A}_{O} \Psi\right)^{(s)}\right\|_{\mathcal{L}_{(\mathrm{sc})}^{2}\left(H_{u}^{(0, \underline{u})}\right)} \lesssim C \delta^{1 / 4}
$$

Indeed the projections of $\left[O, e_{4}\right]$ and $\left[O, e_{3}\right]$ on $e_{3}$ and $e_{4}$, respectively, depend only on $O$ and the Ricci coefficients $\omega, \eta, \underline{\omega}$ and $\underline{\eta}$, while $\left[O, e_{a}\right], a=1,2$, are tangent to $S(u, \underline{u})$. On the other hand, $\mathbb{L}_{O} \Psi^{(s)}$ differs from $\left(\nabla_{O} \Psi\right)^{(s)}$ by terms quadratic in $\nabla O$ and $\Psi$. We recall that $\|\nabla O\|_{\mathcal{L}_{(\mathrm{sc})}^{\infty}} \lesssim C$, i.e. they are regular in the supremum norm. Thus, as before,

$$
\left\|\mathcal{A}_{O} \Psi^{(s)}-\left(\nabla_{O} \Psi\right)^{(s)}\right\|_{\mathcal{L}_{(\mathrm{sc})}^{2}\left(H_{u}^{(0, \underline{u})}\right)} \lesssim C \delta^{1 / 4} .
$$

Combining this with the estimate above and recalling the definition of $\hat{\mathcal{L}}_{O} R$, as well as the estimate $\left\|^{(O)} \pi\right\|_{\mathcal{L}_{(\mathrm{sc})}^{\infty}} \lesssim C$, we derive, for all $s \geqslant \frac{1}{2}$, that

$$
\left\|\Psi^{(s)}\left(\hat{\mathcal{L}}_{O} R\right)-\left(\nabla_{O} \Psi\right)^{(s)}\right\|_{\mathcal{L}_{(\mathrm{sc})}^{2}\left(H_{u}^{(0, \underline{u})}\right)} \lesssim C \delta^{1 / 4}
$$

Similarly we prove, for $s \leqslant \frac{3}{2}$, that

$$
\left\|\Psi^{(s)}\left(\hat{\mathcal{L}}_{O} R\right)-\left(\nabla_{O} \Psi\right)^{(s)}\right\|_{\mathcal{L}_{(\mathrm{sc})}^{2}\left(\underline{H}_{\underline{u}}^{(0, u)}\right)} \lesssim C \delta^{1 / 4}
$$

\subsection{Estimate for $\left\|\nabla_{4} \alpha\right\|_{\mathcal{L}_{(\mathrm{sc})}^{2}(H)}$}

It is important to observe throughout this section that the deformation tensor ${ }^{(L)} \pi$ of $L$ does not contain $\omega$, and similarly ${ }^{(\underline{L})} \pi$ does not contain $\underline{\omega}$. 
We apply Corollary 14.3 to $O=L$ and $X=Y=Z=e_{4}$, and obtain

$$
\begin{gathered}
\int_{H_{u}^{(0, \underline{u})}}\left|\alpha\left(\hat{\mathcal{L}}_{L} R\right)\right|^{2} \lesssim \int_{H_{0}^{(0, \underline{u})}}\left|\alpha\left(\hat{\mathcal{L}}_{L} R\right)\right|^{2}+\int_{\mathcal{D}(u, \underline{u})}\left(Q\left[\hat{\mathcal{L}}_{L} R\right] \cdot{ }^{(4)} \pi\right)\left(e_{4}, e_{4}, e_{4}\right) \\
+\int_{\mathcal{D}(u, \underline{u})} D(L, R)\left(e_{4}, e_{4}, e_{4}\right) .
\end{gathered}
$$

In view of the conservation of signature, we can write schematically

$$
\begin{aligned}
\left(Q\left[\hat{\mathcal{L}}_{L} R\right] \cdot{ }^{(4)} \pi\right)\left(e_{4}, e_{4}, e_{4}\right) & =\sum_{s_{1}+s_{2}+s_{3}=6} \phi^{\left(s_{1}\right)} \Psi^{\left(s_{2}\right)}\left[\hat{\mathcal{L}}_{4} R\right] \Psi^{\left(s_{3}\right)}\left[\hat{\mathcal{L}}_{4} R\right] \\
D(L, R)\left(e_{4}, e_{4}, e_{4}\right) & =\sum_{s_{1}+s_{2}+s_{3}=6} \Psi^{\left(s_{2}\right)}\left[\hat{\mathcal{L}}_{4} R\right]\left(\psi^{\left(s_{1}\right)}(D \Psi)^{\left(s_{3}\right)}+(D \psi)^{\left(s_{1}\right)} \Psi^{\left(s_{3}\right)}\right),
\end{aligned}
$$

with Ricci coefficients $\phi \in\{\chi, \omega, \eta, \underline{\omega}, \underline{\eta}\}$ and $\psi \in\{\chi, \eta, \underline{\omega}, \underline{\eta}\}$, null curvature components $\Psi$ and labels $s_{1}, s_{2}$ and $s_{3}$ denoting the signatures of the corresponding components. Thus,

$$
\left\|\alpha\left(\hat{\mathcal{L}}_{L} R\right)\right\|_{\mathcal{L}_{(\mathrm{sc})}^{2}\left(H_{u}^{(0, \underline{u})}\right)}^{2} \lesssim\left\|\alpha\left(\hat{\mathcal{L}}_{L} R\right)\right\|_{\mathcal{L}_{(\mathrm{sc})}^{2}\left(H_{0}^{(0, \underline{u})}\right)}^{2}+I_{1}+I_{2}+I_{3}
$$

where

$$
\begin{aligned}
& I_{1}=\delta^{1 / 2} \sum_{s_{1}+s_{2}+s_{3}=6}\left\|\phi^{\left(s_{1}\right)}\right\|_{\mathcal{L}_{(\mathrm{sc})}^{\infty}} \int_{0}^{u}\left\|\Psi^{\left(s_{2}\right)}\left(\hat{\mathcal{L}}_{L} R\right)\right\|_{\mathcal{L}_{(\mathrm{sc})}^{2}\left(H_{u^{\prime}}^{(0, \underline{u})}\right)}\left\|\Psi^{\left(s_{3}\right)}\left(\hat{\mathcal{L}}_{4} R\right)\right\|_{\mathcal{L}_{(\mathrm{sc})}^{2}\left(H_{u^{\prime}}^{(0, \underline{u})}\right)} d u^{\prime} \\
& I_{2}=\delta^{1 / 2} \sum_{s_{1}+s_{2}+s_{3}=6}\left\|\psi^{\left(s_{1}\right)}\right\|_{\mathcal{L}_{(\mathrm{sc})}^{\infty}} \int_{0}^{u}\left\|\Psi^{\left(s_{2}\right)}\left(\hat{\mathcal{L}}_{L} R\right)\right\|_{\mathcal{L}_{(\mathrm{sc})}^{2}\left(H_{u^{\prime}}^{(0, \underline{u})}\right)}\left\|(D \Psi)^{\left(s_{3}\right)}\right\|_{\mathcal{L}_{(\mathrm{sc})}^{2}\left(H_{u^{\prime}}^{(0, \underline{u})}\right)} d u^{\prime} \\
& I_{3}=\sum_{s_{1}+s_{2}+s_{3}=6} \int_{0}^{u}\left\|\Psi^{\left(s_{2}\right)}\left(\hat{\mathcal{L}}_{L} R\right)\right\|_{\mathcal{L}_{(\mathrm{sc})}^{2}\left(H_{u^{\prime}}^{(0, \underline{u})}\right)}\left\|(D \psi)^{\left(s_{1}\right)} \Psi^{\left(s_{3}\right)}\right\|_{\mathcal{L}_{(\mathrm{sc})}^{2}\left(H_{u^{\prime}}^{(0, \underline{u})}\right)} d u^{\prime} .
\end{aligned}
$$

Among the terms $I_{1}$, the worst are those in which $s_{2}=s_{3}=3$, in which case $s_{1}=0$. As $\operatorname{tr} \underline{\chi}$ cannot appear among our Ricci coefficients here, and $\|\phi\|_{\mathcal{L}_{(\mathrm{sc})}^{\infty}} \lesssim C$, with $C=C\left(\mathcal{I}^{0}, \mathcal{R}, \underline{\mathcal{R}}\right)$, we have

$$
I_{11} \lesssim C \delta^{1 / 2} \int_{0}^{u}\left\|\alpha\left(\hat{\mathcal{L}}_{L} R\right)\right\|_{\mathcal{L}_{(\mathrm{sc})}^{2}\left(H_{u^{\prime}}^{(0, \underline{u})}\right)}^{2} d u^{\prime}
$$

All curvature terms $\left\|\Psi^{(s)}\left(\hat{\mathcal{L}}_{L} R\right)\right\|_{\mathcal{L}_{(\mathrm{sc})}^{2}\left(H_{u}^{(0, \underline{u})}\right)}$ with $s<3$ can be estimated according to Lemmas 15.1 and 15.3 to derive that

$$
\left\|\Psi^{(s)}\left[\hat{\mathcal{L}}_{L} R\right]\right\|_{\mathcal{L}_{(\mathrm{sc})}^{2}\left(H_{u}\right)} \lesssim \mathcal{R}_{0}+\delta^{1 / 4} C \lesssim C, \quad s<3 .
$$

Therefore, estimating all remaining terms in $I_{1}$, we deduce that

$$
I_{1}(u, \underline{u}) \lesssim C \delta^{1 / 2} \int_{0}^{u}\left(\left\|\alpha\left(\hat{\mathcal{L}}_{L} R\right)\right\|_{\mathcal{L}_{(\mathrm{sc})}^{2}\left(H_{u^{\prime}}^{(0, \underline{u})}\right)}^{2}+\left\|\alpha\left(\hat{\mathcal{L}}_{L} R\right)\right\|_{\mathcal{L}_{(\mathrm{sc})}^{2}\left(H_{u^{\prime}}^{(0, \underline{u})}\right)} \mathcal{R}\right) d u^{\prime}+\delta^{1 / 2} \mathcal{R}^{2} .
$$


The term $I_{2}$ can be estimated in exactly the same manner. Since $0 \leqslant s_{1} \leqslant 1$ and $1 \leqslant s_{2} \leqslant 3$, we have $2 \leqslant s_{3} \leqslant 3$. This implies that the term $(D \Psi)^{s_{3}}$ may be estimated along $H_{u}$. With the exception of the term $\alpha\left(D_{L} R\right)$, these estimates are given in Lemma 15.1. Among those, there are two anomalous terms $\alpha\left(D_{3} R\right)$ and $\beta\left(D_{a} R\right)$. We then obtain

$$
\begin{aligned}
& I_{2}(u, \underline{u}) \\
& \lesssim C \delta^{1 / 2} \int_{0}^{u}\left(\left\|\alpha\left(\hat{\mathcal{L}}_{L} R\right)\right\|_{\mathcal{L}_{(\mathrm{sc})}^{2}\left(H_{u^{\prime}}^{(0, \underline{u})}\right)}^{2}+\left(C \delta^{-1 / 4}+\mathcal{I}^{(0)} \delta^{-1 / 2}\right)\left\|\alpha\left(\hat{\mathcal{L}}_{L} R\right)\right\|_{\mathcal{L}_{(\mathrm{sc})}^{2}\left(H_{u^{\prime}}^{(0, u}\right)}\right) d u^{\prime} \\
& +\mathcal{I}^{(0)} \delta^{-1 / 2}+C \delta^{-1 / 4} \\
& \lesssim C \delta^{1 / 2} \int_{0}^{u}\left\|\alpha\left(\hat{\mathcal{L}}_{L} R\right)\right\|_{\mathcal{L}_{(\mathrm{sc})}^{2}\left(H_{u^{\prime}}^{(0, u)}\right)}^{2} d u^{\prime}+\mathcal{I}^{(0)} \delta^{-1 / 2}+C \delta^{-1 / 4}
\end{aligned}
$$

It remains to estimate $I_{3}$. We note that, in the worst case, the term $D \psi$ can be written in the form

$$
(D \psi)^{\left(s_{1}\right)}=(\nabla \psi)^{s_{1}}+\left(\operatorname{tr} \underline{\chi}_{0}\right) \psi^{\left(s_{1}\right)}+\sum_{s_{11}+s_{12}=s_{1}} \psi^{\left(s_{11}\right)} \psi^{\left(s_{12}\right)} .
$$

Observe that $(\nabla \psi)^{s_{1}} \neq\left(\nabla_{4} \omega, \nabla_{3} \underline{\omega}\right)$. Indeed $\nabla_{4} \omega$ cannot occur, since $\psi^{\left(s_{1}\right)} \in\{\chi, \eta, \underline{\omega}, \underline{\eta}\}$. On the other hand, $\nabla_{3} \underline{\omega}$ cannot occur, by signature considerations. Indeed in that case $s_{1}=\operatorname{sgn}\left(\nabla_{3} \underline{\omega}\right)=0$, which is ruled out since $s_{1}+s_{2}+s_{3}=6$ while $s_{2} \leqslant 3$ and $s_{3} \leqslant 2$.

Thus, since $(\nabla \psi)^{s_{1}} \neq\left(\nabla_{4} \omega, \nabla_{3} \underline{\omega}\right)$ (for which we do not have $\mathcal{L}_{(\mathrm{sc})}^{4}$ estimates!), we derive

$$
\begin{aligned}
& \left\|(D \psi)^{\left(s_{1}\right)} \Psi^{\left(s_{3}\right)}\right\|_{\mathcal{L}_{(\mathrm{sc})}^{2}\left(H_{u^{\prime}}^{(0, \underline{u})}\right)} \\
& \quad \lesssim \delta^{1 / 2}\left\|(\nabla \psi)^{\left(s_{1}\right)}\right\|_{\mathcal{L}_{(\mathrm{sc})}^{4}\left(H_{u^{\prime}}^{(0, \underline{u})}\right)}\left\|\Psi^{\left(s_{3}\right)}\right\|_{\mathcal{L}_{(\mathrm{sc})}^{4}\left(H_{u^{\prime}}^{(0, \underline{u})}\right)} \\
& \quad+\left(\delta \sum_{s_{11}+s_{12}=s_{1}}\left\|\psi^{\left(s_{11}\right)}\right\|_{\mathcal{L}_{(\mathrm{sc})}^{\infty}}\left\|\phi^{\left(s_{12}\right)}\right\|_{\left.\mathcal{L}_{(\mathrm{sc})}^{\infty}+\delta^{1 / 2}\left\|\psi^{\left(s_{1}\right)}\right\|_{\left.\mathcal{L}_{(\mathrm{sc})}^{\infty}\right)}\right)\left\|\Psi^{\left(s_{3}\right)}\right\|_{\mathcal{L}_{(\mathrm{sc})}^{2}\left(H_{u^{\prime}}^{(0, \underline{u})}\right)}}\right. \\
& \quad \lesssim C
\end{aligned}
$$

Observe that in the last step we have used the $\mathcal{L}_{(\mathrm{sc})}^{4}$ estimates for the first derivatives of the Ricci coefficients $\psi \in\{\chi, \eta, \underline{\eta}\}$ and the null curvature components, and allowed for the worst possible scenario in which $\Psi^{\left(s_{3}\right)}=\alpha$,

$$
\begin{aligned}
&\left\|(\nabla \psi)^{\left(s_{1}\right)}\right\|_{\mathcal{L}_{(\mathrm{sc})}^{4}\left(H_{u^{\prime}}^{(0, \underline{u})}\right)}+\left\|\Psi^{\left(s_{3}\right)}\right\|_{\mathcal{L}_{(\mathrm{sc})}^{4}\left(H_{u^{\prime}}^{(0, \underline{u})}\right)} \leqslant C \delta^{-1 / 4}, \\
&\left\|\Psi^{\left(s_{3}\right)}\right\|_{\mathcal{L}_{(\mathrm{sc})}^{2}\left(H_{u^{\prime}}^{(0, \underline{u})}\right)} \lesssim C \delta^{-1 / 2} .
\end{aligned}
$$

As a consequence, we derive that

$$
I_{3}(u, \underline{u}) \lesssim C \int_{0}^{u}\left\|\alpha\left(\hat{\mathcal{L}}_{4} R\right)\right\|_{\mathcal{L}_{(\mathrm{sc})}^{2}\left(H_{u^{\prime}}^{(0, \underline{u})}\right)} d u^{\prime}+C .
$$


Combining the estimates for $I_{1}, I_{2}$ and $I_{3}$, we derive that

$$
\begin{aligned}
& \left\|\alpha\left(\hat{\mathcal{L}}_{4} R\right)\right\|_{\mathcal{L}_{(\mathrm{sc})}^{2}\left(H_{u}^{(0, \underline{u})}\right)}^{2} \\
& \quad \lesssim\left\|\alpha\left(\hat{\mathcal{L}}_{4} R\right)\right\|_{\mathcal{L}_{(\mathrm{sc})}^{2}\left(H_{0}^{(0, \underline{u})}\right)}^{2}+C\left(1+\delta^{1 / 2}\right) \int_{0}^{u}\left\|\alpha\left(\hat{\mathcal{L}}_{4} R\right)\right\|_{\mathcal{L}_{(\mathrm{sc})}^{2}\left(H_{u^{\prime}}^{0, \underline{u})}\right)} d u^{\prime}+C \delta^{1 / 2} .
\end{aligned}
$$

Therefore, in view of the anomalous character of $\left\|\alpha\left(\hat{\mathcal{L}}_{L} R\right)\right\|_{\mathcal{L}_{(\mathrm{sc})}^{2}\left(H_{u}\right)}$,

$$
\delta\left\|\alpha\left(\hat{\mathcal{L}}_{L} R\right)\right\|_{\mathcal{L}_{(\mathrm{sc})}^{2}\left(H_{u}^{(0, \underline{u})}\right)}^{2} \lesssim \delta\left\|\alpha\left(\hat{\mathcal{L}}_{L} R\right)\right\|_{\mathcal{L}_{(\mathrm{sc})}^{2}\left(H_{0}^{(0, \underline{u})}\right)}^{2}+C \delta^{3 / 2}
$$

from which we infer that, for some $C=C\left(\mathcal{I}^{0}, \mathcal{R}, \underline{\mathcal{R}}\right)$,

$$
\delta^{1 / 2}\left\|\alpha\left(\hat{\mathcal{L}}_{L} R\right)\right\|_{\mathcal{L}_{(\mathrm{sc})}^{2}\left(H_{u}^{(0, \underline{u})}\right)} \lesssim \delta^{1 / 2}\left\|\alpha\left(\hat{\mathcal{L}}_{L} R\right)\right\|_{\mathcal{L}_{(\mathrm{sc})}^{2}\left(H_{0}^{(0, \underline{u})}\right)}+C \delta^{1 / 2} \lesssim \mathcal{I}^{0}+C \delta^{1 / 2}
$$

On the other hand, in view of the definition of $\hat{\mathcal{L}}_{L} R$, we have

$$
\alpha\left(\hat{\mathcal{L}}_{L} R\right)=\nabla_{L} \alpha+\sum_{s_{1}+s_{2}=3} \phi^{\left(s_{1}\right)} \Psi^{\left(s_{2}\right)} .
$$

Hence,

$$
\left\|\nabla_{4} \alpha\right\|_{\mathcal{L}_{(\mathrm{sc})}^{2}\left(H_{u}^{(0, \underline{u})}\right)} \lesssim\left\|\alpha\left(\hat{\mathcal{L}}_{L} R\right)\right\|_{\mathcal{L}_{(\mathrm{sc})}^{2}\left(H_{u}^{(0, \underline{u})}\right)}+C \mathcal{R}_{0}
$$

Therefore we deduce the following result.

Proposition 15.4. The following estimate holds true for sufficiently small $\delta>0$, with a constant $C=C\left(\mathcal{I}^{0}, \mathcal{R}, \underline{\mathcal{R}}\right)$ :

$$
\left\|\nabla_{4} \alpha\right\|_{\mathcal{L}_{(\mathrm{sc})}^{2}\left(H_{u}^{(0, \underline{u})}\right)} \lesssim \delta^{-1 / 2} \mathcal{I}^{0}+C .
$$

\subsection{Estimate for $\left\|\nabla_{3} \underline{\alpha}\right\|_{\mathcal{L}_{(\mathrm{sc})}^{2}(H)}$}

Applying Corollary 14.3 to $O=e_{3}$ and $X=Y=Z=e_{3}$, we derive

$$
\begin{aligned}
\int_{\underline{H}_{\underline{u}}^{(0, u)}}\left|\underline{\alpha}\left(\hat{\mathcal{L}}_{\underline{L}} R\right)\right|^{2} \lesssim & \int_{\underline{H}_{0}^{(0, u)}}\left|\underline{\alpha}\left(\hat{\mathcal{L}}_{\underline{L}} R\right)\right|^{2}+\int_{\underline{H}_{0}^{(0, u)}}\left|\underline{\beta}\left(\hat{\mathcal{L}}_{\underline{L}} R\right)\right|^{2} \\
& +\int_{\mathcal{D}(u, \underline{u})}\left(Q\left[\hat{\mathcal{L}}_{\underline{L}} R\right] \cdot{ }^{(3)} \pi\right)\left(e_{3}, e_{3}, e_{3}\right)+\int_{\mathcal{D}(u, \underline{u})} D(\underline{L}, R)\left(e_{3}, e_{3}, e_{3}\right) .
\end{aligned}
$$

In view of the conservation of signature we can write schematically (we need to take into account the signature associated with the integrals)

$$
\begin{aligned}
\left(Q\left[\hat{\mathcal{L}}_{\underline{L}} R\right] \cdot{ }^{(3)} \pi\right)\left(e_{3}, e_{3}, e_{3}\right) & =\sum_{s_{1}+s_{2}+s_{3}=1} \psi^{\left(s_{1}\right)} \Psi^{\left(s_{2}\right)}\left[\hat{\mathcal{L}}_{3} R\right] \Psi^{\left(s_{3}\right)}\left[\hat{\mathcal{L}}_{3} R\right] \\
D(\underline{L}, R)\left(e_{3}, e_{3}, e_{3}\right) & =\sum_{s_{1}+s_{2}+s_{3}=1} \Psi^{\left(s_{2}\right)}\left[\hat{\mathcal{L}}_{\underline{L}} R\right]\left(\psi^{\left(s_{1}\right)}(D \Psi)^{\left(s_{3}\right)}+(D \psi)^{\left(s_{1}\right)} \Psi^{\left(s_{3}\right)}\right),
\end{aligned}
$$


with Ricci coefficients $\psi \in\{\omega, \eta, \underline{\chi}, \underline{\eta}\}$, null curvature components $\Psi$ and labels $s_{1}, s_{2}$ and $s_{3}$ denoting the signatures of the corresponding components. We now need to be careful with terms which involve $\operatorname{tr} \underline{\chi}$ and $\nabla_{3} \operatorname{tr} \underline{\chi}$. In (15.16) the only terms which contain $\operatorname{tr} \underline{\chi}$ have the form $(\operatorname{tr} \underline{\chi})\left|\underline{\beta}\left(\hat{\mathcal{L}}_{\underline{L}} R\right)\right|^{2}$ which we write in the form

$$
\left(\operatorname{tr} \underline{\chi}_{0}\right)\left|\underline{\beta}\left(\hat{\mathcal{L}}_{\underline{L}} R\right)\right|^{2}+(\widetilde{\operatorname{tr} \underline{\chi}})\left|\underline{\beta}\left(\hat{\mathcal{L}}_{\underline{L}} R\right)\right|^{2} .
$$

In (15.17) the only terms which contain $\nabla_{3} \operatorname{tr} \underline{\chi}$ must be of the form

$$
\nabla_{3}(\operatorname{tr} \underline{\chi}) \Psi^{\left(s_{2}\right)}\left(\hat{\mathcal{L}}_{\underline{L}} R\right) \Psi^{\left(s_{3}\right)}, \quad s_{2}+s_{3}=1 .
$$

Recall that

$$
\nabla_{3} \operatorname{tr} \underline{\chi}=-\frac{1}{2}(\operatorname{tr} \underline{\chi})^{2}-2 \underline{\omega} \operatorname{tr} \underline{\chi}-|\underline{\widehat{\chi}}|^{2} .
$$

Thus, writing $\operatorname{tr} \underline{\chi}=\operatorname{tr} \underline{\chi}_{0}+\widetilde{\operatorname{tr}} \underline{\chi}$, we schematically have

$$
\nabla_{3} \operatorname{tr} \underline{\chi}=-\frac{1}{2}\left(\operatorname{tr} \underline{\chi}_{0}\right)^{2}+\left(\operatorname{tr} \underline{\chi}_{0}\right) \psi_{g}+\psi \psi .
$$

We have

$$
\left\|\underline{\alpha}\left(\hat{\mathcal{L}}_{\underline{L}} R\right)\right\|_{\mathcal{L}_{\text {(sc) }}^{2}\left(H_{\underline{u}}^{(0, u)}\right)}^{2} \lesssim\left\|\underline{\alpha}\left(\hat{\mathcal{L}}_{\underline{L}} R\right)\right\|_{\mathcal{L}_{\text {(sc) }}^{2}\left(H_{0}^{(0, u)}\right)}^{2}+P_{1}+P_{2}+P_{3}+J_{1}+J_{2}+J_{3},
$$

where $P_{1}, P_{2}$ and $P_{3}$ are the terms corresponding to the terms in $\operatorname{tr} \underline{\chi}_{0}$ :

$$
\begin{aligned}
& P_{1}=\sum_{s_{2}+s_{3}=1} \delta^{-1} \int_{0}^{\underline{u}}\left\|\Psi^{\left(s_{2}\right)}\left(\hat{\mathcal{L}}_{\underline{L}} R\right)\right\|_{\mathcal{L}_{(\mathrm{sc})}^{2}\left(\underline{H}_{\underline{u}^{\prime}}^{(0, u)}\right)}\left\|\Psi^{\left(s_{3}\right)}\left(\hat{\mathcal{L}}_{\underline{L}} R\right)\right\|_{\mathcal{L}_{(\mathrm{sc})}^{2}\left(\underline{H}_{\underline{u}^{\prime}}^{(0, u)}\right)} d \underline{u}^{\prime}, \\
& P_{2}=\sum_{s_{2}+s_{3}=1} \delta^{-1} \int_{0}^{\underline{u}}\left\|\Psi^{\left(s_{2}\right)}\left(\hat{\mathcal{L}}_{\underline{L}} R\right)\right\|_{\mathcal{L}_{(\mathrm{sc})}^{2}\left(\underline{H}_{\underline{u}^{\prime}}^{(0, u)}\right)}\left\|(D \Psi)^{\left(s_{3}\right)}\right\|_{\mathcal{L}_{(\mathrm{sc})}^{2}\left(\underline{H}_{\underline{u}^{\prime}}^{(0, u)}\right)} d \underline{u}^{\prime}, \\
& P_{3}=\sum_{s_{2}+s_{3}=1} \delta^{-1} \int_{0}^{\underline{u}}\left\|\Psi^{\left(s_{2}\right)}\left(\hat{\mathcal{L}}_{3} R\right)\right\|_{\mathcal{L}_{(\mathrm{sc})}^{2}\left(\underline{H}_{\underline{u}^{\prime}}^{(0, u)}\right)}\left\|\Psi^{\left(s_{3}\right)}\right\|_{\mathcal{L}_{(\mathrm{sc})}^{2}\left(\underline{H}_{\underline{u}^{\prime}}^{(0, u)}\right)} d \underline{u}^{\prime},
\end{aligned}
$$

and $J_{1}, J_{2}$ and $J_{3}$ are the remaining terms with Ricci coefficients $\psi \in\{\eta, \underline{\widehat{\chi}}, \underline{\eta}\}$ :

$$
\begin{aligned}
J_{1}= & \delta^{-1 / 2} \sum_{s_{1}+s_{2}+s_{3}=1}\left\|\psi^{\left(s_{1}\right)}\right\|_{\mathcal{L}_{\text {(sc) }}^{\infty}} \\
& \times \int_{0}^{\underline{u}}\left\|\Psi^{\left(s_{2}\right)}\left(\hat{\mathcal{L}}_{3} R\right)\right\|_{\mathcal{L}_{\text {(sc) }}^{2}\left(\underline{H}_{\underline{u}^{\prime}}^{(0, u)}\right)}\left\|\Psi^{\left(s_{3}\right)}\left(\hat{\mathcal{L}}_{3} R\right)\right\|_{\mathcal{L}_{(\mathrm{sc})}^{2}\left(\underline{H}_{\underline{u}^{\prime}}^{(0, u)}\right)} d \underline{u}^{\prime}, \\
J_{2}= & \delta^{-1 / 2} \sum_{s_{1}+s_{2}+s_{3}=1}\left\|\psi^{\left(s_{1}\right)}\right\|_{\mathcal{L}_{\text {(sc) }}^{\infty}} \int_{0}^{\underline{u}}\left\|\Psi^{\left(s_{2}\right)}\left(\hat{\mathcal{L}}_{3} R\right)\right\|_{\mathcal{L}_{(\mathrm{sc})}^{2}\left(\underline{H}_{\underline{u}^{\prime}}^{(0, u)}\right)}\left\|(D \Psi)^{\left(s_{3}\right)}\right\|_{\mathcal{L}_{(\mathrm{sc})}^{2}\left(\underline{H}_{\underline{u}^{\prime}}^{(0, u)}\right)} d \underline{u}^{\prime}, \\
J_{3}= & \sum_{s_{1}+s_{2}+s_{3}=1} \delta^{-1} \int_{0}^{\underline{u}}\left\|\Psi^{\left(s_{2}\right)}\left(\hat{\mathcal{L}}_{3} R\right)\right\|_{\mathcal{L}_{(\mathrm{sc})}^{2}\left(\underline{H}_{\underline{u}^{\prime}}^{(0, u)}\right)}\left\|(D \psi)^{\left(s_{1}\right)} \Psi^{\left(s_{3}\right)}\right\|_{\mathcal{L}_{\text {(sc) }}^{2}\left(\underline{H}_{\underline{u}^{\prime}}^{(0, u)}\right)} d \underline{u}^{\prime} .
\end{aligned}
$$


It clearly suffices to estimate the principal terms $P$. Indeed, the $J$ terms can be treated exactly as in the previous subsection. $\left({ }^{26}\right)$ We have

$$
P_{1} \lesssim \delta^{-1} \int_{0}^{\underline{u}}\left\|\underline{\beta}\left(\hat{\mathcal{L}}_{\underline{L}} R\right)\right\|_{\mathcal{L}_{(\mathrm{sc})}^{2}\left(\underline{H}_{\underline{u}^{\prime}}^{(0, u)}\right)}^{2} d \underline{u}^{\prime} .
$$

According to Lemma 15.3, we have

$$
\left\|\underline{\beta}\left(\hat{\mathcal{L}}_{\underline{L}} R\right)\right\|_{\mathcal{L}_{(\mathrm{sc})}^{2}\left(\underline{H}_{\underline{u}^{\prime}}^{(0, u)}\right)}^{2} \lesssim \| \nabla_{3} \underline{\beta}_{\mathcal{L}_{(\mathrm{sc})}^{2}\left(\underline{H}_{\underline{u}^{\prime}}^{(0, u)}\right)}^{2}+\underline{\mathcal{R}}_{0}+\delta^{1 / 4} C .
$$

In view of the Bianchi identities, for $\frac{1}{2} \leqslant s \leqslant 1$,

$$
\nabla_{3} \underline{\beta}=\operatorname{div} \underline{\alpha}-2(\operatorname{tr} \underline{\chi}) \underline{\beta}-2 \underline{\omega} \underline{\beta}+\underline{\eta} \underline{\alpha} .
$$

Therefore,

$$
\left\|\underline{\beta}\left(\underline{\mathcal{L}}_{\underline{L}} R\right)\right\|_{\mathcal{L}_{(\mathrm{sc})}^{2}\left(\underline{H}_{\underline{u}^{\prime}}^{(0, u)}\right)}^{2} \lesssim\|\nabla \underline{\alpha}\|_{\mathcal{L}_{(\mathrm{sc})}^{2}\left(\underline{H}_{\underline{u}^{\prime}}^{(0, u)}\right)}^{2}+\underline{\mathcal{R}}_{0}+\delta^{1 / 4} C
$$

Consequently,

$$
\begin{aligned}
P_{1}(u, \underline{u}) & \lesssim \delta^{-1} \int_{0}^{\underline{u}}\left(\|\nabla \underline{\alpha}\|_{\mathcal{L}_{(\mathrm{sc})}^{2}\left(\underline{H}_{\underline{u}^{\prime}}^{(0, u)}\right)}^{2}+\underline{\mathcal{R}}_{0}\left(u, \underline{u}^{\prime}\right)\right) d \underline{u}^{\prime}+C \delta^{1 / 4} \\
& \lesssim \delta^{-1} \int_{0}^{\underline{u}} \underline{\mathcal{R}}^{2}\left(u, \underline{u}^{\prime}\right) d \underline{u}^{\prime}+\delta^{1 / 4} C .
\end{aligned}
$$

The terms $P_{2}$ and $P_{3}$ can be estimated exactly in the same manner. First, observe that in $P_{2}$ the terms of the form $(D \Psi)^{\left(s_{3}\right)}$ obey the bounds

$$
\left\|(D \Psi)^{\left(s_{3}\right)}\right\|_{\mathcal{L}_{(\mathrm{sc})}^{2}\left(\underline{H}_{\underline{u}^{\prime}}^{(0, u)}\right)} \lesssim \underline{\mathcal{R}}\left(u, \underline{u}^{\prime}\right)+\delta^{1 / 4} C .
$$

This follows from the restriction $s_{3} \leqslant 1$. Similarly, for $s_{2} \leqslant 1$,

$$
\left\|\Psi^{\left(s_{2}\right)}\left(\hat{\mathcal{L}}_{\underline{L}} R\right)\right\|_{\mathcal{L}_{(\mathrm{sc})}^{2}\left(\underline{H}_{\underline{u}^{\prime}}^{(0, u)}\right)} \lesssim\left\|\underline{\alpha}\left(\hat{\mathcal{L}}_{\underline{L}} R\right)\right\|_{\mathcal{L}_{(\mathrm{sc})}^{2}\left(\underline{H}_{\underline{u}^{\prime}}^{(0, u)}\right)}+\underline{\mathcal{R}}\left(u, \underline{u}^{\prime}\right)+\delta^{1 / 2} C .
$$

Therefore,

$$
P_{2}(u, \underline{u}) \lesssim \delta^{-1} \int_{0}^{\underline{u}}\left\|\underline{\alpha}\left(\hat{\mathcal{L}}_{\underline{L}} R\right)\right\|_{\mathcal{L}_{(\mathrm{sc})}^{2}\left(\underline{H}_{\underline{u}^{\prime}}^{(0, u)}\right)}^{2} d \underline{u}^{\prime}+\delta^{-1} \int_{0}^{\underline{u}} \underline{\mathcal{R}}^{2}\left(u, \underline{u}^{\prime}\right) d \underline{u}^{\prime}+\delta^{1 / 2} C .
$$

Similarly,

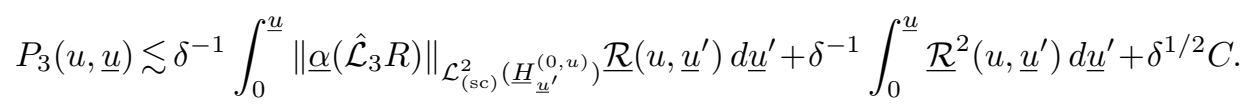

Therefore, using Lemma 15.3, we derive the following result.

Proposition 15.5. The following estimate holds true for sufficiently small $\delta>0$, with a constant $C=C\left(\mathcal{I}^{0}, \mathcal{R}, \underline{\mathcal{R}}\right)$ :

$$
\left\|\nabla_{3} \underline{\alpha}_{\mathcal{L}_{(\mathrm{sc})}^{2}\left(\underline{H}_{\underline{u}}^{(0, u)}\right)}^{2} \lesssim\right\| \nabla_{3} \underline{\alpha} \|_{\mathcal{L}_{(\mathrm{sc})}^{2}\left(\underline{H}_{0}^{(0, u)}\right)}^{2}+\underline{\mathcal{R}}+\delta^{-1} \int_{0}^{\underline{u}} \underline{\mathcal{R}}\left(u, \underline{u}^{\prime}\right)^{2} d \underline{u}^{\prime}+\delta^{1 / 4} C .
$$

$\left({ }^{26}\right)$ Remark that, in $J_{2},(D \Psi)^{(3)}$ differs from $\nabla_{3} \underline{\omega}$, because $\Psi^{\left(s_{3}\right)} \in\{\omega, \eta, \underline{\chi}, \underline{\eta}\}$, and from $\nabla_{4} \omega$, by signature considerations. 


\subsection{Estimates for the angular derivatives of $R$}

Applying Corollary 14.3 to the angular momentum vector fields $O$ and $X, Y, Z \in\left\{e_{3}, e_{4}\right\}$, we derive

$$
\begin{aligned}
\int_{H_{u}^{(0, \underline{u})}} & \left|\Psi^{(s)}\left(\hat{\mathcal{L}}_{O} R\right)\right|^{2}+\int_{H_{\underline{u}}^{(0, u)}}\left|\Psi^{(s-1 / 2)}\left(\hat{\mathcal{L}}_{O} R\right)\right|^{2} \\
& \lesssim \int_{H_{0}^{(0, \underline{u})}}\left|\Psi^{(s)}\left(\hat{\mathcal{L}}_{O} R\right)\right|^{2}+\int_{\mathcal{D}(u, \underline{u})}\left(Q\left[\hat{\mathcal{L}}_{O} R\right] \cdot \pi\right)(X, Y, Z)+\int_{\mathcal{D}(u, \underline{u})} D(O, R)(X, Y, Z) .
\end{aligned}
$$

In view of the conservation of signature, we can write schematically

$$
\begin{aligned}
\left(Q\left[\hat{\mathcal{L}}_{O} R\right] \cdot \pi\right)(X, Y, Z)=\left(\operatorname{tr} \underline{\chi}_{0}\right) \sum_{s_{2}+s_{3}=2 s} \Psi^{\left(s_{2}\right)}\left[\hat{\mathcal{L}}_{O} R\right] \Psi^{\left(s_{3}\right)}\left[\hat{\mathcal{L}}_{O} R\right] \\
+\sum_{s_{1}+s_{2}+s_{3}=2 s} \phi^{\left(s_{1}\right)} \Psi^{\left(s_{2}\right)}\left[\hat{\mathcal{L}}_{O} R\right] \Psi^{\left(s_{3}\right)}\left[\hat{\mathcal{L}}_{O} R\right],
\end{aligned}
$$

with a Ricci coefficient $\phi \in\{\chi, \omega, \eta, \underline{\widehat{\chi}}, \widetilde{\operatorname{tr}} \underline{\chi}, \underline{\omega}, \underline{\eta}\}$. Also, recalling that $\pi=\hat{\pi}+\frac{1}{4}(\operatorname{tr} \pi) g$,

$$
D(O, R)(X, Y, Z)=\sum_{s_{1}+s_{2}+s_{3}=2 s} \Psi^{\left(s_{2}\right)}\left[\hat{\mathcal{L}}_{O} R\right]\left({ }^{(O)} \pi^{\left(s_{1}\right)}(D \Psi)^{\left(s_{3}\right)}+\left(D^{(O)} \pi\right)^{\left(s_{1}\right)} \Psi^{\left(s_{3}\right)}\right)
$$

with ${ }^{(O)} \pi^{(s)}$ being the null components of the deformation tensor of $O$. Thus, for all $s>\frac{1}{2}$,

$$
\begin{aligned}
\left\|\Psi^{(s)}\left(\hat{\mathcal{L}}_{O} R\right)\right\|_{\mathcal{L}_{(\mathrm{sc})}^{2}\left(H_{u}^{(0, \underline{u})}\right)}^{2}+ & \left\|\Psi^{(s-1 / 2)}\left(\hat{\mathcal{L}}_{O} R\right)\right\|_{\mathcal{L}_{(\mathrm{sc})}^{2}\left(H_{\underline{u}}^{(0, u)}\right)}^{2} \\
& \lesssim\left\|\Psi^{(s)}\left(\hat{\mathcal{L}}_{O} R\right)\right\|_{\mathcal{L}_{(\mathrm{sc})}^{2}\left(H_{0}^{(0, \underline{u})}\right)}^{2}+I_{1}+I_{2}+I_{3}
\end{aligned}
$$

where

- $I_{1}$ is the integral in $\mathcal{D}(u, \underline{u})$ whose integrand is given by (15.19),

- $I_{2}$ is the integral in $\mathcal{D}(u, \underline{u})$ whose integrand is given by

$$
\sum_{s_{1}+s_{2}+s_{3}=2 s} \Psi^{\left(s_{2}\right)}\left[\hat{\mathcal{L}}_{O} R\right]^{(O)} \pi^{\left(s_{1}\right)}(D \Psi)^{\left(s_{3}\right)},
$$

- $I_{3}$ is the integral in $\mathcal{D}(u, \underline{u})$ whose integrand is given by

$$
\sum_{s_{1}+s_{2}+s_{3}=2 s} \Psi^{\left(s_{2}\right)}\left[\hat{\mathcal{L}}_{O} R\right]\left(D^{(O)} \pi\right)^{\left(s_{1}\right)} \Psi^{\left(s_{3}\right)} .
$$

In what follows we make use of the estimate for the deformation tensors of the angular momentum vector fields established in Theorem 13.7, that is

$$
\left\|^{(O)} \pi\right\|_{\mathcal{L}_{(\mathrm{sc})}^{4}(S)}+\left\|^{(O)} \pi\right\|_{\mathcal{L}_{\text {(sc) }}^{\infty}(S)} \lesssim C .
$$


Also, all null components of the derivatives $D^{(O)} \pi$, with the exception of $\left(D_{3}{ }^{(O)} \pi\right)_{3 a}$, satisfy the estimates

$$
\left\|D^{(O)} \pi\right\|_{\mathcal{L}_{(\mathrm{sc})}^{4}(S)} \lesssim C
$$

Moreover,

$$
\left\|\left(D_{3}{ }^{(O)} \pi\right)_{3 a}-\nabla_{3} Z\right\|_{L^{4}(S)}+\left\|\sup _{\underline{u}}\left|\nabla_{3} Z\right|\right\|_{L^{2}(S)} \lesssim C .
$$

The term $I_{1}$ can be easily estimated, since none of the curvature terms are anomalous. Indeed, in view of Lemma 15.3, we have, for all $s>\frac{1}{2}$,

$$
\begin{aligned}
\left\|\Psi^{(s)}\left(\hat{\mathcal{L}}_{O} R\right)\right\|_{\mathcal{L}_{(\mathrm{sc})}^{2}\left(H_{u}^{(0, \underline{u})}\right)} & \lesssim\left\|\Psi^{(s)}\left(\hat{\mathcal{L}}_{O} R\right)-\nabla_{O} \Psi^{(s)}\right\|_{\mathcal{L}_{(\mathrm{sc})}^{2}\left(H_{u}^{(0, \underline{u})}\right)}+\left\|\nabla_{O} \Psi^{(s)}\right\|_{\mathcal{L}_{(\mathrm{sc})}^{2}\left(H_{u}^{(0, \underline{u})}\right)} \\
& \lesssim \mathcal{R}(u, \underline{u})
\end{aligned}
$$

while, for $s=\frac{1}{2}$,

$$
\left\|\Psi^{(1 / 2)}\left(\hat{\mathcal{L}}_{O} R\right)\right\|_{\mathcal{L}_{(\mathrm{sc})}^{2}\left(\underline{H}_{\underline{u}}^{(0, u)}\right)} \lesssim \underline{\mathcal{R}}(u, \underline{u}) .
$$

Consequently, for $s>\frac{1}{2}$,

$$
I_{1} \lesssim \sum_{s \geqslant 1} \int_{0}^{u}\left\|\Psi^{(s)}\left(\hat{\mathcal{L}}_{O} R\right)\right\|_{\mathcal{L}_{\text {(sc) }}^{2}\left(H_{u^{\prime}}^{(0, \underline{u})}\right)}^{2} d u^{\prime}+\delta^{1 / 2} C,
$$

while, for $s=\frac{1}{2}$,

$$
I_{1} \lesssim \sum_{s \leqslant 2} \delta^{-1} \int_{0}^{\underline{u}}\left\|\Psi^{(s)}\left(\hat{\mathcal{L}}_{O} R\right)\right\|_{\mathcal{L}_{(\mathrm{sc})}^{2}\left(H_{\underline{u}^{\prime}}^{(0, u)}\right)}^{2} d \underline{u}^{\prime}+\delta^{1 / 2} C .
$$

Therefore,

$$
\begin{aligned}
I_{1} \lesssim \sum_{s \geqslant 1} & \int_{0}^{u}\left\|\Psi^{(s)}\left(\hat{\mathcal{L}}_{O} R\right)\right\|_{\mathcal{L}_{(\mathrm{sc})}^{2}\left(H_{u^{\prime}}^{(0, \underline{u})}\right)}^{2} d u^{\prime} \\
& +\sum_{s \leqslant 2} \delta^{-1} \int_{0}^{\underline{u}}\left\|\Psi^{(s)}\left(\hat{\mathcal{L}}_{O} R\right)\right\|_{\mathcal{L}_{(\mathrm{sc})}^{2}\left(H_{\underline{u}^{\prime}}^{(0, u)}\right)}^{2} d \underline{u}^{\prime}+\delta^{1 / 2} C .
\end{aligned}
$$

Among the terms $I_{2}$, the only possible anomalies may occur in the case $s_{3}=3$, i.e. $(D \Psi)^{\left(s_{3}\right)}=\alpha\left(D_{4} R\right)$, or in the easier cases $(D \Psi)^{\left(s_{3}\right)}=\alpha\left(D_{3} R\right)$ and $(D \Psi)^{\left(s_{3}\right)}=\beta\left(D_{a} R\right)$ (i.e. $s_{3}=2$ ). We denote by $I_{21}$ all terms in $I_{2}$ except those which correspond to these anomalous cases. For all other terms we have either

$$
\left\|(D \Psi)^{\left(s_{3}\right)}\right\|_{\mathcal{L}_{(\mathrm{sc})}^{2}\left(H_{\underline{u}^{\prime}}^{(0, u)}\right)} \lesssim C \quad \text { or } \quad\left\|(D \Psi)^{\left(s_{3}\right)}\right\|_{\mathcal{L}_{(\mathrm{sc})}^{2}\left(\underline{H}_{u^{\prime}}^{(0, \underline{u})}\right)} \lesssim C
$$


Using also $\left\|{ }^{(O)} \pi\right\|_{\mathcal{L}_{\text {(sc) }}^{\infty}} \lesssim C$ and

$$
\begin{aligned}
\left\|\Psi^{\left(s_{2}\right)}\left(\hat{\mathcal{L}}_{O} R\right)\right\|_{\mathcal{L}_{(\mathrm{sc})}\left(H_{u}^{(0, \underline{u})}\right)} \lesssim C, \quad s_{2} \geqslant 1, \\
\left\|\Psi^{\left(s_{2}\right)}\left(\hat{\mathcal{L}}_{O} R\right)\right\|_{\mathcal{L}_{(\mathrm{sc})}^{2}\left(H_{\underline{u}}^{(0, u)}\right)} \lesssim C, \quad s_{2} \leqslant 2,
\end{aligned}
$$

we derive that

$$
I_{21} \lesssim \delta^{1 / 4} C
$$

We now consider the terms $I_{22}$ which contain $(D \Psi)^{\left(s_{3}\right)} \alpha\left(D_{3} R\right)$ and $(D \Psi)^{\left(s_{3}\right)}=\beta\left(D_{a} R\right)$, but not $\alpha\left(D_{4} R\right)$. In this case we write, according to Remark 15.2 ,

$$
\begin{aligned}
(D \Psi)^{\left(s_{3}\right)} & =G+F^{\left(s_{3}\right)}, & & \\
\left\|F^{\left(s_{3}\right)}\right\|_{\mathcal{L}_{(\mathrm{sc})}^{2}\left(H_{u}^{(0, \underline{u})}\right)} & \lesssim C, & & s_{3}>1, \\
\left\|F^{\left(s_{3}\right)}\right\|_{\mathcal{L}_{(\mathrm{sc})}^{2}\left(\underline{H}_{\underline{H}}^{(0, u)}\right)} & \lesssim C, & & s_{3}<2,
\end{aligned}
$$

where $G=\left(\operatorname{tr} \underline{\chi}_{0}\right) \alpha$. Clearly, the terms corresponding to $F^{\left(s_{3}\right)}$ can be estimated exactly as above. To estimate the terms corresponding to $G$, we make use of the $\mathcal{L}_{(\mathrm{sc})}^{4}(S)$ estimate $\|G\|_{\mathcal{L}_{(\mathrm{sc})}^{4}(S)} \leqslant C \delta^{-1 / 4}$. Using also that $\left\|^{(O)} \pi\right\|_{\mathcal{L}_{(\mathrm{sc})}^{4}(S)} \lesssim C$, we obtain

$$
I_{22} \lesssim \delta^{1 / 4} C
$$

It remains to estimate the terms in $I_{23}$ which contain $\alpha\left(D_{4} R\right)$. The integrand which contains $\alpha\left(D_{4} R\right)$ has the form

$$
D_{23}=\sum_{s_{1}+s_{2}=2 s-3}{ }^{(O)} \pi^{\left(s_{1}\right)} \Psi^{\left(s_{2}\right)}\left(\hat{\mathcal{L}}_{O} R\right) \alpha\left(D_{4} R\right) .
$$

This term is potentially dangerous! In view of Lemma $15.3, \Psi^{\left(s_{2}\right)}\left(\hat{\mathcal{L}}_{O} R\right)$ differs from $\left(\nabla_{O} \Psi\right)^{\left(s_{2}\right)}$ by lower-order terms. It thus suffices to estimate

$$
D_{23} \equiv \sum_{s_{1}+s_{2}=2 s-3}{ }^{(O)} \pi^{\left(s_{1}\right)}\left(\nabla_{O} \Psi\right)^{\left(s_{2}\right)} \alpha\left(D_{4} R\right)
$$

We also decompose

$$
\alpha\left(D_{4} R\right)=\nabla_{4} \alpha+\sum_{s_{3}+s_{4}=3} \phi^{\left(s_{3}\right)} \Psi^{\left(s_{4}\right)},
$$

where $\phi^{\left(s_{3}\right)} \in\{\omega, \eta, \underline{\eta}\}$. This forces $s_{4}<2$ and hence, since there are no anomalies, we derive that

$$
\left\|\alpha\left(D_{4} R\right)-\nabla_{4} \alpha\right\|_{\mathcal{L}_{(\mathrm{sc})}^{2}\left(H_{u}^{(0, \underline{u})}\right)} \lesssim C \delta^{1 / 2} .
$$


Therefore, we can safely replace $\alpha\left(D_{4} R\right)$ by $\nabla_{4} \alpha$, and hence it remains to estimate

$$
D_{23} \equiv \sum_{s_{1}+s_{2}=2 s-3}{ }^{(O)} \pi^{\left(s_{1}\right)}\left(\nabla_{O} \Psi\right)^{\left(s_{2}\right)} \nabla_{4} \alpha
$$

Because of the anomaly of $\nabla_{4} \alpha$, the best we can do by a straightforward estimate is to derive an estimate of the form $I_{23} \lesssim \mathcal{I}^{(0)}+C$, which is not acceptable. Because of this, we are forced to integrate by parts, ignoring the boundary term

$$
\int_{\underline{H}_{\underline{u}}}^{(O)} \pi^{\left(s_{1}\right)}\left(\nabla_{O} \Psi\right)^{\left(s_{2}\right)} \alpha
$$

for a moment, obtaining

$$
\begin{aligned}
\int_{\mathcal{D}}{ }^{(O)} \pi^{\left(s_{1}\right)}\left(\nabla_{O} \Psi\right)^{\left(s_{2}\right)} \nabla_{4} \alpha \\
\quad=-\int_{\mathcal{D}}\left(\nabla_{4}{ }^{(O)} \pi^{\left(s_{1}\right)}\right)\left(\nabla_{O} \Psi\right)^{\left(s_{2}\right)} \alpha-\int_{\mathcal{D}}{ }^{(O)} \pi^{\left(s_{1}\right)}\left(\nabla_{4}\left(\nabla_{O} \Psi\right)^{\left(s_{2}\right)}\right) \alpha .
\end{aligned}
$$

We write schematically, with $\phi^{(1 / 2)} \in\{\eta, \underline{\eta}\}$,

$$
\begin{aligned}
\nabla_{4}\left(\nabla_{O} \Psi\right)^{\left(s_{2}\right)} & =\nabla_{4} \nabla_{O}(\Psi)^{\left(s_{2}-1 / 2\right)} \\
& =\nabla_{O} \nabla_{4}(\Psi)^{\left(s_{2}-1 / 2\right)}+\sum_{s_{3}+s_{4}=s_{2}+1} \Psi^{\left(s_{3}\right)} \Psi^{\left(s_{4}\right)}+\sum_{s_{4}=s_{2}+1 / 2} \phi^{(1 / 2)} \Psi^{\left(s_{4}\right)} .
\end{aligned}
$$

We can therefore replace the integrand $D_{23}$ by

$$
D_{23} \equiv-D_{231}-D_{232}-D_{233}-D_{234}
$$

where

$$
\begin{aligned}
D_{231} & =\sum_{s_{1}+s_{2}=2 s-3}\left(\nabla_{4}{ }^{(O)} \pi^{\left(s_{1}\right)}\right)\left(\nabla_{O} \Psi\right)^{\left(s_{2}\right)} \alpha \\
D_{232} & =\sum_{s_{1}+s_{2}=2 s-3}{ }^{(O)} \pi^{\left(s_{1}\right)}\left(\nabla_{O} \nabla_{4} \Psi^{\left(s_{2}+1 / 2\right)}\right) \alpha \\
D_{233} & =\sum_{s_{1}+s_{2}=2 s-3}{ }^{(O)} \pi^{\left(s_{1}\right)}\left(\sum_{s_{3}+s_{4}=s_{2}+1} \Psi^{\left(s_{3}\right)} \Psi^{\left(s_{4}\right)}\right) \alpha \\
D_{234} & =\sum_{s_{1}+s_{2}=2 s-3}{ }^{(O)} \pi^{\left(s_{1}\right)}\left(\sum_{s_{4}=s_{2}+1 / 2} \phi^{(1 / 2)} \Psi^{\left(s_{4}\right)}\right) \alpha .
\end{aligned}
$$

Accordingly, we decompose $I_{23} \equiv I_{231}+I_{232}+I_{233}+I_{234}$. Now,

$$
I_{231} \lesssim \delta^{1 / 2}\left\|\nabla_{4}{ }^{(O)} \pi^{\left(s_{1}\right)}\right\|_{\mathcal{L}_{(\mathrm{sc})}^{4}(S)}\|\alpha\|_{\mathcal{L}_{(\mathrm{sc})}^{4}(S)} \delta^{-1} \int_{0}^{\underline{u}}\left\|\left(\nabla_{O} \Psi\right)^{\left(s_{2}\right)}\right\|_{\mathcal{L}_{(\mathrm{sc})}\left(H_{\underline{u}^{\prime}}^{(0, u)}\right)} d \underline{u}^{\prime} \lesssim \delta^{1 / 4} C .
$$


The terms $I_{233}$ and $I_{234}$ are clearly of lower order in $\delta$, and thus we derive that

$$
I_{233}+I_{234} \lesssim \delta^{1 / 2} C
$$

It remains to estimate $I_{232}$, for which we need to perform another integration by parts. We write

$$
\begin{aligned}
\int_{\mathcal{D}}{ }^{(O)} \pi^{\left(s_{1}\right)}\left(\nabla_{O} \nabla_{4} \Psi^{\left(s_{2}+1 / 2\right)}\right) \alpha=- & \int_{\mathcal{D}}\left(\nabla_{O}{ }^{(O)} \pi^{\left(s_{1}\right)}\right)\left(\nabla_{4} \Psi^{\left(s_{2}+1 / 2\right)}\right) \alpha \\
& -\int_{\mathcal{D}}{ }^{(O)} \pi^{\left(s_{1}\right)}\left(\nabla_{4} \Psi^{\left(s_{2}+1 / 2\right)}\right) \nabla_{O} \alpha \\
& -\int_{\mathcal{D}}{ }^{(O)} \pi^{\left(s_{1}\right)}\left(\nabla_{4} \Psi^{\left(s_{2}+1 / 2\right)}\right)\left(\nabla^{a} O_{a}\right) \alpha
\end{aligned}
$$

By the Bianchi identities, since $s_{2}+\frac{1}{2}<3$,

$$
\left\|\left(\nabla_{4} \Psi\right)^{\left(s_{2}+1 / 2\right)}\right\|_{\mathcal{L}_{(\mathrm{sc})}\left(H_{u}^{(0, \underline{u})}\right)} \lesssim\left\|(\nabla \Psi)^{\left(s_{2}+1 / 2\right)}\right\|_{\mathcal{L}_{(\mathrm{sc})}\left(H_{u}^{(0, \underline{u})}\right)}+\delta^{1 / 2}\|\phi\|_{\mathcal{L}_{(\mathrm{sc})}^{\infty}}\|\Psi\|_{\mathcal{L}_{(\mathrm{sc})}^{2}(S)} \leqslant C .
$$

Therefore,

$$
\begin{aligned}
& \left|\int_{\mathcal{D}}\left(\nabla_{O}{ }^{(O)} \pi^{\left(s_{1}\right)}\right)\left(\nabla_{4} \Psi\right)^{\left(s_{2}+1 / 2\right)} \alpha\right| \\
& \quad \lesssim \delta^{1 / 2}\left\|\nabla_{O}{ }^{(O)} \pi^{\left(s_{1}\right)}\right\|_{\mathcal{L}_{(\mathrm{sc})}^{4}(S)}\|\alpha\|_{\mathcal{L}_{(\mathrm{sc})}^{4}(S)} \int_{0}^{u}\left\|\left(\nabla_{4} \Psi\right)^{\left(s_{2}+1 / 2\right)}\right\|_{\mathcal{L}_{(\mathrm{sc})}\left(H_{u^{\prime}}^{(0, \underline{u})}\right)} d u^{\prime} \lesssim \delta^{1 / 4} C .
\end{aligned}
$$

Also,

$$
\begin{aligned}
& \left|\int_{\mathcal{D}}{ }^{(O)} \pi^{\left(s_{1}\right)}\left(\nabla_{4} \Psi\right)^{\left(s_{2}+1 / 2\right)} \nabla_{O} \alpha\right| \\
& \quad \lesssim \delta^{1 / 2} \int_{0}^{u}\left\|\nabla_{O} \alpha\right\|_{\mathcal{L}_{(\mathrm{sc})}\left(H_{u^{\prime}}^{(0, \underline{u})}\right)}\left\|\left(\nabla_{4} \Psi\right)^{\left(s_{2}+1 / 2\right)}\right\|_{\mathcal{L}_{(\mathrm{sc})}\left(H_{u^{\prime}}^{(0, \underline{u})}\right)} d u^{\prime}\left\|{ }^{(O)} \pi^{\left(s_{1}\right)}\right\|_{\mathcal{L}_{(\mathrm{sc})}^{\infty}(S)} \lesssim \delta^{1 / 2} C .
\end{aligned}
$$

The remaining integral in $I_{232}$ is clearly of lower order in $\delta$. For the boundary term in (15.22), we have

$$
\begin{aligned}
\left|\int_{\underline{H}_{\underline{u}}}{ }^{(O)} \pi^{\left(s_{1}\right)}\left(\nabla_{O} \Psi\right)^{\left(s_{2}\right)} \alpha\right| & \lesssim \delta^{1 / 2}\left\|\left(\nabla_{O} \Psi\right)^{\left(s_{2}\right)}\right\|_{\mathcal{L}_{(\mathrm{sc})}^{2}\left(\underline{H}_{\underline{u}}\right)}\left\|{ }^{(O)} \pi\right\|_{\mathcal{L}_{(\mathrm{sc})}^{4}(S)}\|\alpha\|_{\mathcal{L}_{(\mathrm{sc})}^{4}(S)} \\
& \leqslant \delta^{1 / 4} C .
\end{aligned}
$$

We therefore deduce that

$$
I_{2} \lesssim C \delta^{1 / 4}
$$

Consider now $I_{3}$. Ignoring powers of $\delta$, we have to estimate the integral

$$
\int_{\mathcal{D}}\left(D^{(O)} \pi\right)^{\left(s_{1}\right)} \Psi^{\left(s_{2}\right)}\left(\hat{\mathcal{L}}_{O} R\right) \Psi^{\left(s_{3}\right)} .
$$


Recall the estimates

$$
\left\|\left(D^{(O)} \pi\right)^{\left(s_{1}\right)}\right\|_{\mathcal{L}_{(\mathrm{sc})}^{4}(S)} \lesssim C
$$

for all components of $\left(D^{(O)} \pi\right)^{\left(s_{1}\right)}$, with the exception of the term $D_{3}{ }^{(O)} \pi_{3 a}$ which corresponds to the signature $s_{1}=0$. In this latter case, we have

$$
\left\|D_{3}{ }^{(O)} \pi_{3 a}-\nabla_{3} Z\right\|_{\mathcal{L}_{(\mathrm{sc})}^{4}(S)} \lesssim C \quad \text { and } \quad\left\|\sup _{\underline{u}}\left|\left(D_{3}{ }^{(O)} \pi\right)_{3 a}\right|\right\|_{\mathcal{L}_{(\mathrm{sc})}^{2}(S)} \lesssim C .
$$

In the case $\left(D^{(O)} \pi\right)^{\left(s_{1}\right)} \neq D_{3}{ }^{(O)} \pi_{3 a}$, we have

$$
\begin{aligned}
& \left|\int_{\mathcal{D}}\left(D^{(O)} \pi\right)^{\left(s_{1}\right)} \Psi^{\left(s_{2}\right)}\left(\hat{\mathcal{L}}_{O} R\right) \Psi^{\left(s_{3}\right)}\right| \\
& \quad \lesssim \delta^{1 / 2} \delta^{-1} \int_{0}^{\underline{u}}\left\|\left(\nabla_{O} \Psi\right)^{\left(s_{2}\right)}\right\|_{\mathcal{L}_{(\mathrm{sc})}^{2}\left(H_{\underline{u}^{\prime}}^{(0, u)}\right)} d \underline{u}^{\prime}\left\|\left(D^{(O)} \pi\right)^{\left(s_{1}\right)}\right\|_{\mathcal{L}_{(\mathrm{sc})}^{4}(S)}\left\|\Psi^{\left(s_{3}\right)}\right\|_{\mathcal{L}_{(\mathrm{sc})}^{4}(S)} \\
& \quad \leqslant \delta^{1 / 4} C
\end{aligned}
$$

where we considered the worst case in which $\Psi^{\left(s_{3}\right)}=\alpha$, and thus is anomalous, and $\left(\nabla_{O} \Psi\right)^{\left(s_{2}\right)}$ has to be estimated along $H_{\underline{u}^{\prime}}^{(0, u)}$.

For this case, we can replace, without loss of generality, $\left(D \nabla^{(O)} \pi\right)^{\left(s_{1}\right)}$ by $\nabla_{3} Z$. Indeed, the remaining error term can be estimated exactly as above. In this case, since $s_{1}=0$, signature considerations dictate that $s_{3} \geqslant 1$, as follows from the conditions

$$
s_{1}+s_{2}+s_{3}=2 s, \quad s_{2} \in\left\{s, s-\frac{1}{2}\right\} \quad \text { and } \quad s \geqslant 1
$$

This implies that we may use the trace theorem along $H_{u}$,

$$
\left\|\Psi^{\left(s_{3}\right)}\right\|_{\operatorname{Tr}_{(\mathrm{sc})}(H)} \lesssim \delta^{-1 / 4} C
$$

where in fact $\delta^{-1 / 4}$ only occurs in the case when $\Psi^{\left(s_{3}\right)}=\alpha$, for all other terms the behavior in $\delta$ is better. We thus give the argument only for $\Psi^{\left(s_{3}\right)}=\alpha$, other cases are even easier. Recalling also Lemma 15.3,

$$
\begin{aligned}
& \left|\int_{\mathcal{D}} \nabla_{3} Z \Psi^{\left(s_{2}\right)}\left(\hat{\mathcal{L}}_{O} R\right) \Psi^{\left(s_{3}\right)}\right| \\
& \quad \lesssim \delta^{1 / 2} \delta^{-1} \int_{0}^{\underline{u}}\left\|\left(\nabla_{O} \Psi\right)^{\left(s_{2}\right)}\right\|_{\mathcal{L}_{(\mathrm{sc})}\left(H_{\underline{u}^{\prime}}^{(0, u)}\right)} d \underline{u}^{\prime}\left\|\sup _{\underline{u}}\left|\nabla_{3} Z\right|\right\|_{\mathcal{L}_{(\mathrm{sc})}^{2}(S)} \sup _{u}\left\|\Psi^{\left(s_{3}\right)}\right\|_{\operatorname{Tr}_{(\mathrm{sc})}\left(H_{u}\right)} \\
& \quad \leqslant \delta^{1 / 4} C .
\end{aligned}
$$

We finally observe that the only borderline terms not resulting in positive powers of the parameter $\delta$ and arising from coupling with $\operatorname{tr} \underline{\chi}$, involve only $\beta, \varrho, \sigma$ and $\underline{\beta}$ components of the curvature. 
Combining all our estimates for $I, I_{2}$ and $I_{3}$, and using Lemma 14.5, we derive that

$$
\begin{aligned}
\sum_{1 \leqslant s \leqslant 5 / 2}\left(\left\|\Psi^{(s)}\left(\hat{\mathcal{L}}_{O} R\right)\right\|_{\mathcal{L}_{(\mathrm{sc})}^{2}\left(H_{u}^{(0, \underline{u})}\right)}\right. & \left.+\left\|\Psi^{(s-1 / 2)}\left(\hat{\mathcal{L}}_{O} R\right)\right\|_{\mathcal{L}_{(\mathrm{sc})}^{2}\left(H_{\underline{u}}^{(0, u)}\right)}\right) \\
& \lesssim \sum_{1 \leqslant s \leqslant 2}\left\|\Psi^{(s)}\left(\hat{\mathcal{L}}_{O} R\right)\right\|_{\mathcal{L}_{(\mathrm{sc})}^{2}\left(H_{0}^{(0, \underline{u})}\right)}+\delta^{1 / 4} C .
\end{aligned}
$$

More precisely, we easily check the estimate

$$
\left\|\alpha\left(\hat{\mathcal{L}}_{O} R\right)\right\|_{\mathcal{L}_{(\mathrm{sc})}^{2}\left(H_{u}^{(0, \underline{u})}\right)}+\left\|\beta\left(\hat{\mathcal{L}}_{O} R\right)\right\|_{\mathcal{L}_{(\mathrm{sc})}^{2}\left(H_{\underline{\underline{u}}}^{(0, u)}\right)} \lesssim\left\|\alpha\left(\hat{\mathcal{L}}_{O} R\right)\right\|_{\mathcal{L}_{(\mathrm{sc})}^{2}\left(H_{0}^{(0, \underline{u})}\right)}+\delta^{1 / 4} C .
$$

For $s \leqslant 2$ we have

$$
\begin{aligned}
\sum_{s \leqslant 2}\left(\left\|\Psi^{(s)}\left(\hat{\mathcal{L}}_{O} R\right)\right\|_{\mathcal{L}_{(\mathrm{sc})}^{2}\left(H_{u}^{(0, \underline{u})}\right)}+\left\|\Psi^{(s-1 / 2)}\left(\hat{\mathcal{L}}_{O} R\right)\right\|_{\mathcal{L}_{(\mathrm{sc})}^{2}\left(H_{\underline{u}}^{(0, u)}\right)}\right) & \\
& \lesssim \sum_{s \leqslant 2}\left\|\Psi^{(s)}\left(\hat{\mathcal{L}}_{O} R\right)\right\|_{\mathcal{L}_{(\mathrm{sc})}^{2}\left(H_{0}^{(0, \underline{u})}\right)}+\delta^{1 / 4} C .
\end{aligned}
$$

Using the estimates of Lemma 15.3, we derive that

$$
\|\nabla \alpha\|_{\mathcal{L}_{(\mathrm{sc})}^{2}\left(H_{u}^{(0, \underline{u})}\right)}+\|\nabla \beta\|_{\mathcal{L}_{(\mathrm{sc})}^{2}\left(H_{\underline{u}}^{(0, u)}\right)} \lesssim\|\nabla \alpha\|_{\mathcal{L}_{(\mathrm{sc})}^{2}\left(H_{0}^{(0, \underline{u})}\right)}+\delta^{1 / 4} C .
$$

For $s \leqslant 2$ we have

$$
\begin{aligned}
\sum_{s \leqslant 2}\left(\left\|(\nabla \Psi)^{(s)}\right\|_{\mathcal{L}_{(\mathrm{sc})}^{2}\left(H_{u}^{(0, \underline{u})}\right)}+\left\|(\nabla \Psi)^{(s-1 / 2)}\right\|_{\mathcal{L}_{(\mathrm{sc})}^{2}\left(H_{\underline{u}}^{(0, u)}\right)}\right) & \\
& \lesssim \sum_{s \leqslant 2}\left\|(\nabla \Psi)^{(s)}\right\|_{\mathcal{L}_{(\mathrm{sc})}^{2}\left(H_{0}^{(0, \underline{u})}\right)}+\delta^{1 / 4} C .
\end{aligned}
$$

We summarize the result above in the following proposition.

Proposition 15.6. The following estimates hold true for $\delta$ sufficiently small and $C=C\left(\mathcal{I}^{(0)}, \mathcal{R}, \underline{\mathcal{R}}\right)$ :

$$
\sum_{1 \leqslant s \leqslant 5 / 2}\left(\left\|\nabla \Psi^{(s)}\right\|_{\mathcal{L}_{(\mathrm{sc})}^{2}\left(H_{u}^{(0, \underline{u})}\right)}+\left\|\nabla \Psi^{(s-1 / 2)}\right\|_{\mathcal{L}_{(\mathrm{sc})}^{2}\left(H_{\underline{u}}^{(0, u)}\right)}\right) \lesssim \mathcal{I}_{0}+C \delta^{1 / 4} .
$$

Combining this result with Propositions 15.4 and 15.5, we get

$$
\mathcal{R}_{1}+\underline{\mathcal{R}}_{1} \lesssim \mathcal{I}_{0}+C \delta^{1 / 4}
$$

Finally, combining this with Proposition 14.6, we derive that

$$
\mathcal{R}+\underline{\mathcal{R}} \leqslant \mathcal{I}_{0}+C \delta^{1 / 4} .
$$

This ends the proof of our main theorem. 


\subsection{Proof of Propositions 2.5 and 2.6}

The proof of Proposition 2.5 is an immediate consequence of estimate (15.24) together with the initial assumptions derived in Proposition 2.4. Indeed, under initial assumptions (2.8), we derive that

$$
\sum_{s \leqslant 2}\left(\left\|(\nabla \Psi)^{(s)}\right\|_{\mathcal{L}_{\text {(sc) }}^{2}\left(H_{u}^{(0, \underline{u})}\right)}+\left\|(\nabla \Psi)^{(s-1 / 2)}\right\|_{\mathcal{L}_{(\mathrm{sc})}^{2}\left(H_{\underline{u}}^{(0, u)}\right)}\right) \lesssim \varepsilon+\delta^{1 / 4} C
$$

which gives, for sufficiently small $\delta$, estimate (2.9).

We combine this result with Proposition 11.8 to prove the following scale-invariant version of Proposition 2.6 in the introduction.

Proposition 15.7. The solution ${ }^{(3)} \phi$ of the problem $\nabla_{3}^{(3)} \phi=\nabla \eta$ with trivial initial data satisfies

$$
\left\|{ }^{(3)} \phi\right\|_{\mathcal{L}_{\text {(sc) }}^{\infty}(S)} \leqslant C \varepsilon^{1 / 4}+C \delta^{1 / 16}
$$

\section{References}

[C] Christodoulou, D., The Formation of Black Holes in General Relativity. EMS Monographs in Mathematics. Eur. Math. Soc., Zürich, 2009.

[CK] Christodoulou, D. \& Klainerman, S., The Global Nonlinear Stability of the Minkowski Space. Princeton Mathematical Series, 41. Princeton University Press, Princeton, NJ, 1993.

[KN] Klainerman, S. \& Nicolò, F., The Evolution Problem in General Relativity. Progress in Mathematical Physics, 25. Birkhäuser, Boston, MA, 2003.

[KR1] Klainerman, S. \& Rodnianski, I., Causal geometry of Einstein-vacuum spacetimes with finite curvature flux. Invent. Math., 159 (2005), 437-529.

[KR2] - A geometric approach to the Littlewood-Paley theory. Geom. Funct. Anal., 16 (2006), $126-163$

[RT] Reiterer, M. \& Trubowitz, E., Strongly focused gravitational waves. Comm. Math. Phys., 307 (2011), 275-313.

Sergiu Klainerman

Department of Mathematics

Princeton University

Princeton, NJ 08544

U.S.A.

seri@math.princeton.edu
IGOR RODNIANSKI

Department of Mathematics

Princeton University

Princeton, NJ 08544

U.S.A.

irod@math.princeton.edu

Received February 10, 2010 